\title{
Kapitel 14 \\ Zunftsungewisse Vorausdeutungen
}

\section{Einleitung}

Für die Rekonstruktion von paulinischen Protonarrativen stellt die bisher erörterte Kategorie der Behauptungen über zukünftige Ereignisse narrative Fixpunkte bereit. Das heißt: Sie schließen aus, dass es zu einem bestimmten Zeitpunkt in der Zukunft möglich sein wird, bestimmte Protoerzählungen (wahrheitsgemäß) als explizite Erzählungen zu äußern - nämlich solche, die diese Ereignisse diserzählen würden. ${ }^{1}$ Neben zukunftsgewissen Vorausdeutungen des Erzählers auf Geschehnisse der vor ihm liegenden Zukunft spielen jedoch auch zukunftsungewisse Vorausdeutungen auf denselben Zeitabschnitt eine wichtige, wenn auch etwas anders gelagert Rolle. Diese variiert zudem gehörig, je nachdem, welches konkrete sprachliche Mittel vom Autor gewählt wird, um auf ein eventuell stattfindendes zukünftiges Ereignis Bezug zu nehmen. Dies betrifft sowohl den Grad an Sicherheit, mit welchem das Ereignis postuliert wird, als auch die bei den Adressaten bezweckte Wirkung.

\section{Fragesätze}

\subsection{Hinführung}

Unter der Überschrift der zukunftsgewissen Vorausdeutungen wurden selbstständige und abhängige Behauptungssätze diskutiert. Nun soll der Komplex der Fragesätze besprochen werden, denn auch hier können Zukunftsbezüge vorliegen. Anders als im Behauptungssatz wird hierbei jedoch nicht eine Aussage über ein zukünftiges Geschehen gemacht. Das Ereignis wird durch die Frage jedoch hypothetisch aufgegriffen und in das Zentrum der Aufmerksamkeit gerückt. In diesem Abschnitt wird zunächst ein Überblick über die formalen Gestaltungsmöglichkeiten von Fragesätzen und dann deren Bedeutung für zukunftsungewisse Vorausdeutungen geboten.

Im Fall von (1) Entscheidungsfragen ${ }^{2}$ wird das Vorliegen eines Zustands oder Ereignisses so erfragt, dass als Antwort eine einfache Bejahung oder Verneinung

1 Genauer gesagt: Sie schließen solche Protoerzählungen aus, denen kein Plot entnommen werden kann, in welchen diese Ereignisse integriert werden könnten.

2 Vgl. fürs Deutsche Duden 1394. 
genügt. ${ }^{3}$ Die Sache, über die eine Entscheidung gefällt wird, ist durch die Frage selbst also bereits angesprochen, wobei je nach Formulierung eine unterschiedlich starke Eingrenzung vorgenommen wird. ${ }^{4}$

Formal (d. h. abgesehen vom erst zu byzantinischer Zeit verwendeten Fragezeichen „;“) $)^{5}$ ist die Entscheidungsfrage mit einem Behauptungssatz identisch. Die Fragepartikel $\tilde{\alpha} \rho \alpha$ kann einen Satz jedoch als Frage kennzeichnen. ${ }^{6}{ }_{\alpha}^{\tilde{\alpha}} \rho \alpha$ erscheint allerdings bei Paulus nur in Gal 2,17: „Ist Christus Diener der Sünde?“

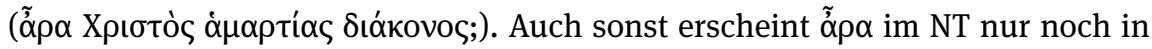
Lk 18,8 und Mt 12,10. ${ }^{7}$

Häufiger ist im NT der eigentümliche Gebrauch von عỉ als Fragepartikel. ${ }^{8}$ Hierbei ergibt sich freilich ein Klassifizierungsproblem, da auch abhängige Fragesätze mit عi („ob“) eingeleitet werden. ${ }^{9}$ Abhängige Fragesätze mit ci (d. h. indirekte Entscheidungsfragen) sind bei Paulus recht selten. ${ }^{10}$

Da Tempus und Modus ${ }^{11}$ im Griechischen (anders als im Deutschen und Englischen) in der indirekten Rede erhalten bleiben, stellen diese Parameter kein Unterscheidungskriterium dar. Von den drei bei GGNT 269b angegebenen Beispielen (Mt 12,10, Lk 13,23, Apg 1,6) liegt nur im letzten Fall eindeutig direkte Rede vor, da hier vom Vokativ Gebrauch gemacht wird und das Verb in der 2. Singular

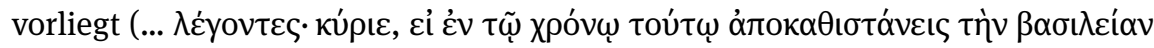
$\left.\tau \tilde{\omega}{ }^{\prime} \operatorname{I} \sigma \rho \alpha \hat{n} \lambda ;\right)$.

Bei Paulus würde der Gebrauch von ci als Fragepartikel die Syntax von 2. Kor 12,15 zufriedenstellend erklären: Dann wäre hier mit cỉ kein Bedingungs-

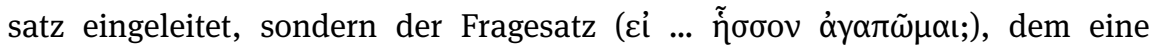

\section{GGNT 269a.}

4 Vgl. auf deutsch: „Wirst Du uns nach dem Umzug in die neue Wohnung besuchen kommen?“ vs. „Wirst Du uns am Samstagabend für einen Spieleabend besuchen kommen?“

5 Vgl. GGNT S. 22 und CGCG 1.13.

6 GGNT 269c.

7 Letztlich bietet sich auch hier kein eindeutiges Differenzierungskriterium zwischen Frage- und Behauptungssatz an, da in den frühen Handschriften natürlich nicht zwischen ã $\rho \alpha$ und ő $\rho \alpha$ unterschieden werden kann. Der Beitrag des „Markers“ ist daher von der Eindeutigkeit der syntaktischen Einbettung abhängig.

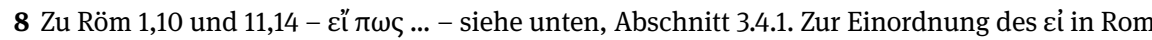
9,22 siehe Kapitel 12, Abschnitt 8.3. Im Römerbrief ist keine der Futurverbformen Teil eines sol-

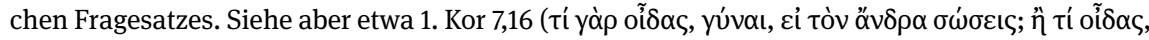

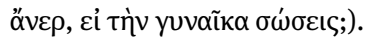

9 Siehe hierzu GGNT 273. Vgl. etwa auch 2. Kor 2,9 Kapitel 13, Abschnitt 3.1.

10 Siehe hierzu GGNT 273a. Für das klassische Griechisch siehe Schwyzer-Debrunner II, 687688. Vgl. Caragounis, Language, 191.

11 Zum obliquen Optativ bei innerlicher Abhängigkeit siehe GGNT 273d. Er ist bei Paulus nicht belegt. 
Partizipialkonstruktion vorangestellt wäre: „Euch mehr liebend, werde ich

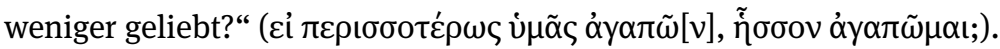

Da hier freilich kein Zukunftsbezug vorliegt, ist diese Entscheidung für die hier verfolgte Fragestellung ohnehin nicht von großer Bedeutung. Generell verdient die Option jedoch wohl mehr Berücksichtigung in der Exegese, als ihr bisher meist zukommt. Dass der Gebrauch von ei als Fragepartikel sonst bei Paulus nicht belegt ist, ist kaum ein Gegenargument, ${ }^{12}$ gilt die Einmaligkeit ja auch im Hinblick für $\tilde{\alpha} \rho \alpha$. Zudem ist es durchaus nicht gesagt, dass 2. Kor 12,15 ein derart isoliert dastehendes Phänomen darstellt.

Der im NT zu beobachtende Gebrauch von عì als Fragepartikel verweist nämlich auf ein grundsätzliches Problem im Hinblick auf die Ergänzung von Lesezeichen in Ausgaben des NT: Die Besonderheit von ci als Fragepartikel ${ }^{13}$ wird meist als hebraisierende Tendenz gedeutet, die auf den Einfluss von Hebräischem אִ und הִ zurückgeht. ${ }^{14}$ Man kann jedoch auch dafür argumentieren, dass hier vielmehr eine orthographische Rationalisierung des nicht mehr richtig verstandenen (da gleich klingenden) $\tilde{\tilde{\eta}}$ zu sehen ist. ${ }^{15}$

Dies verweist natürlich auf eine Folgefrage: Ist $\tilde{\eta}$ überhaupt als Fragepartikel im NT bezeugt? NA ${ }^{28}$ entscheidet sich so an keiner Stelle. Gerade in den Paulusbriefen ist diese Entscheidung jedoch öfters anfechtbar. Dies gilt insbesondere für Fälle, in denen $\eta$ ” (,oder“) eingliedrige Disjunktionsfragen einleiten würde. ${ }^{16}$ So eröffnet in

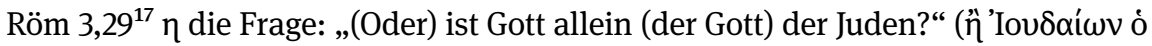

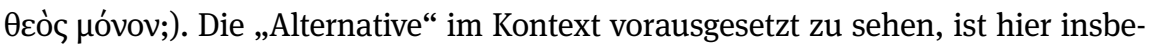
sondere deswegen schwierig, weil diese direkt im Fortgang des Textes erfragt und auch beantwortet wird („Nicht auch der Heiden? Ja, auch der Heiden“; oủxì кà̀

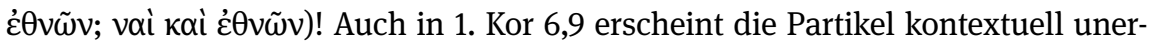

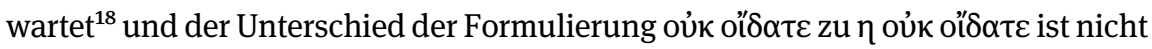
klar ersichtlich. ${ }^{19}$ Besonders stark legt sich eine Akzentuierung als $\tilde{\eta}$ in 1 . Thess 2,19 nahe: „Denn wer ist unsere Hoffnung oder Freude oder Ruhmeskranz - nicht auch

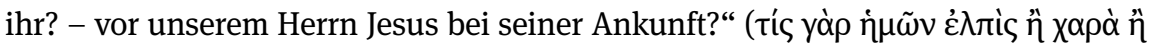

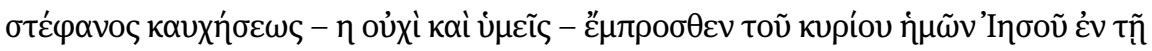

12 Gegen Schmeller, Brief II, 349.

13 Vgl. Schwyer-Debrunner II 628: „Nur indirekt sind عi, ,ob“ usw.“

14 So auch GGNT 269b.

15 Caragounis, Development, 211-216. Vgl. zur hier gebotenen Diskussion auch die aktualisierte Präsentation des Arguments bei Caragounis, Language, 180-208.

16 Siehe GGNT 251b zu Disjunktionspartikeln und 269b zur Disjunktionsfrage.

17 Siehe zu den angeführten Stellen Caragounis, Development, 209-210.

18 Vgl. zur Stelle oben, Kapitel 13, Abschnitt 3.1.

19 Caragounis, Development, 209-210. 


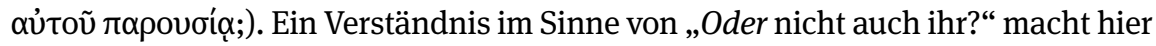
wenig Sinn. ${ }^{20}$ Die Sachlage kann wohl noch nicht als ausreichend aufgearbeitet gelten, die These hat jedoch durchaus einiges für sich. ${ }^{21}$ Eine vollständige Analyse des Materials wäre auf jeden Fall wünschenswert, ${ }^{22}$ auch wenn der Unterschied in der Bedeutung zugegebenermaßen relativ geringfügig ist. ${ }^{23}$

Besonders interessant für die Rolle von Fragesätzen im Hinblick auf die durch sie evozierten Protonarrative sind Fragepartikeln, welche die erwartete Antwort andeuten: So wird durch oủ oder (verstärkt) oủxí die Erwartung einer Bestätigung ausgedrückt, während $\mu$ '́ die Frage als eine verneinende Antwort erfordernd kennzeichnet. ${ }^{24}$ Die tatsächlich gegebene Antwort muss natürlich nicht mit der

20 Vgl. etwa NSS 1097. Caragounis, Development, 209 formuliert besonders stark: „the disjunctive significance ... is grammatically impossible.“ Vgl. den alternativen Vorschlag bei BDR 4461 .

21 Was die Aussprache angeht, so ist gerade $\eta$ der „Zankapfel“ zwischen Vertretern einer „rekonstruierten“ historischen Aussprache (wie R. Buth) und Advokaten der „historischen“ (=„,modernen“) Aussprache wie Caragounis. Zweifellos richtig ist, dass bereits sehr früh und recht weit verbreitet eine Verschiebung des Lautwerts von $\eta \mathrm{zu}$ เ einsetzt: etwa im Pamphylischen, ab dem 3. Jh. v. Chr. im Böotischen, bereits zwischen dem 6. und 4. Jh. im Kyprischen (siehe hierzu Karvounis, Aussprache, 73-74). Auch fürs Attische ist die Lautverschiebung keinesfalls - wie immer wieder behauptet - nur kaiserzeitlich/nachchristlich bezeugt. Die Sichtung des relevanten Materials bringt Karvounis, Aussprache, 77 zur Schlussfolgerung, dass „das epigraphische Material ... diese Interpretation unmöglich“ mache. Dennoch ist zumindest teilweise - und das verkompliziert die von Caragounis vorgetragenen These der „Historical Greek Pronounciation“ ein wenig - eine weitere Realisierung von $\eta$ mit /e/ anzunehmen, eventuell sogar im Falle des Pontischen Dialekts bis ins Neugriechische hinein (vgl. insgesamt Karvounis, Aussprache, 73-77). Das Hauptproblem von Caragounis' Interpretation von ci als Variante von $\tilde{\eta}$ in direkten Fragesätzen ist daher wohl nicht die Lautentwicklung als solche. Erstaunlich ist aber doch gerade, dass er trotz der Sichtung von Hunderten von Vorkommnissen in den Papyri auf keinen Fall verweisen kann, wo عı zur Einleitung einer direkten Frage steht. Wäre die Realisierung des /i/-Lauts als عí nicht auch in den Papyri zu erwarten, wenn im Hintergrund die generelle Ausbreitung von $\varepsilon i$ in das Territorium von $\tilde{\eta}$ stehen würde (so Caragounis, Development, 216)? Eine teilweise Antwort mag Caragounis' weitere Beobachtung liefern, dass ci auch für indirekte Fragen in den Papyri sehr selten ist (er zählt 6 Vorkommnisse bei Caragounis, Development, 212).

$22 \mathrm{Zu}$ erwägen wäre beispielsweise eine Untersuchung im Rahmen der Synoptiker. Spontan

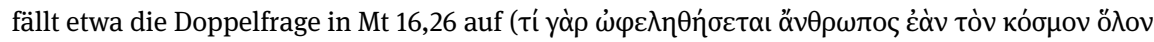

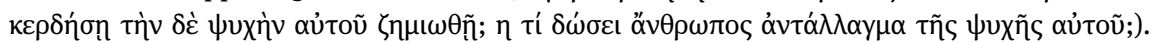
Werden hier, durch $\eta$ " markiert, zwei separate Erwägungen angestellt? Oder wird lediglich im zweiten Fall mit Nachdruck der Gedanke zu Ende geführt? Auffällig ist in jedem Fall, dass bei Mk 8,37 durch einen anderen Konnektor das Verhältnis unzweideutig im Sinne der zweiten Möglich-

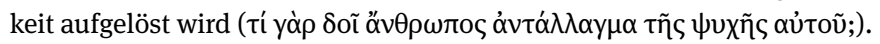

23 Vgl. hierzu Caragounis, Development, 210-211.

24 GGNT 269c. Während $\mu$ ń mit Indikativ den Satz eindeutig als Fragesatz markiert, gilt dies bei oủ/oủxí natürlich nicht. Das bei Schwyzer-Debrunner II, 292-293 zum voluntativen Gebrauch des Ind. Fut. in negierten Fragen Gesagte scheint für die Koine nicht anwendbar zu sein. 
Erwartungshaltung des Fragenden korrespondieren. ${ }^{25}$ (Im Deutschen spielt zur Kommunikation dieses Aspekts die Intonation eine große Rolle.) ${ }^{26}$

Bei (2) Ergänzungsfragen ${ }^{27}$ wird vom Befragten mehr Information eingefordert: Es wird mithilfe eines Fragewortes „nach einer Person, einer Sache oder einem Umstand gefragt, “28 wobei abhängig vom einleitenden Interrogativpronomen/-adverb ${ }^{29}$ das Erfragte aus der Perspektive des Fragenden mehr oder weniger offen ist. Ein entsprechend großer Informationsbeitrag ist vom Antwortenden

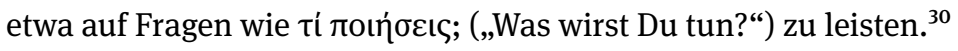

Indirekte Ergänzungsfragen haben entweder dieselben Interrogativpronomen/-adverbien wie entsprechende selbstständige Ergänzungsfragen oder werden durch indirekte Interrogativa und auch Relativpronomina eingeleitet. ${ }^{31}$ Syntaktisch lassen sie sich den Relativsätzen zuordnen. ${ }^{32}$ Vgl. die Diskussion zu 1. Thess 4,1 unten, Abschnitt 4.4.3.1. Eine indikativische Futurform nach Interrogativpronomen außerhalb von direkten Fragesätzen findet sich im corpus Paulinum nur

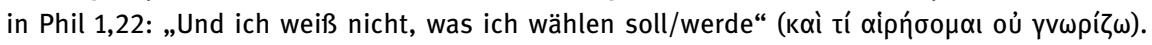

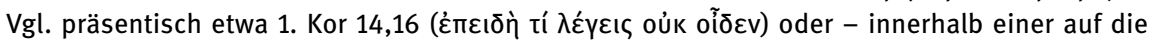

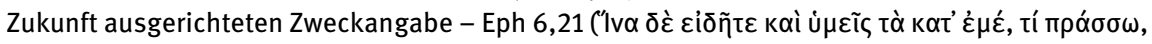

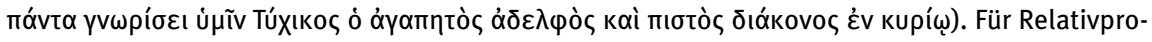
nomina in der Funktion von Interrogativpronomina siehe die parallele Gebrauchsweise in 1. Tim

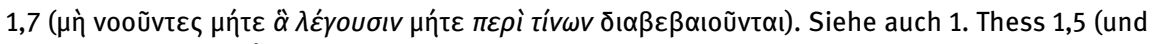
evtl. 2. Tim 3,11) zu oíos. Vgl. 1. Kor 3,13, Gal 2,6,1. Thess 1,9 zu òmог̃os.

Sowohl Entscheidungs- als auch Ergänzungsfragen ${ }^{33}$ können auch als rhetorische Fragen vorliegen, bei denen der Fragende also keine Antwort erwartet, sondern

25 Siehe Schwyzer-Debrunner II 629 mit Verweis auf Mt 26,25.

26 Vgl. im Deutschen allerdings die Kennzeichnung der „Bestätigungsfragesätze“ mit „doch,“ „doch nicht etwa,“ „wohl doch nicht etwa“ (Zifonun, Hoffmann und Strecker, Grammatik I, 643). Zur Intonation siehe Duden 170-177. Zum „Frageton“ im Griechischen siehe Schwyzer-Debrunner II 267 und 269 (neugriechisch).

27 Vgl. fürs Deutsche Duden 1393.

28 GGNT 269a.

29 Vgl. GGNT 269d.

30 Wie groß der Ausschnitt der - potenziellen - Realität ist, auf den durch die Frage Bezug genommen wird, hängt darüber hinaus natürlich auch vom Kontext ab. So kann die Frage nach dem zu Tuenden angesichts eines sehr konkreten Problems geäußert werden, das nur wenige, spezifische Lösungsmöglichkeiten zulässt. Zugleich kann damit sehr allgemein nach dem Handeln innerhalb eines größeren Zeitraumes (z. B. auf die Sommerferien Bezug nehmend) gefragt werden. 31 GGNT 273b. Für Beispiele mit Relativpronomina siehe auch GGNT 142f. Für Beispiele mit Interrogativpronomina siehe auch GGNT 143a. Zum gegenseitigen Ersatz siehe GGNT 143b.

32 Vgl. GGNT 289a.

33 Schon deswegen ist es absurd, dass teilweise in Lehrbüchern die Behauptung aufgestellt wird, eine rhetorische Frage werde „mit einer Negation eingeleitet“ (Schoch, Lehrgang, 66). 
lediglich deswegen einen Fragesatz formuliert, „um den Adressaten zur Anerkennung einer bereits vorhandenen Meinung zu bewegen. “34

Beide Satztypen (ob rhetorisch oder nicht) sind in der Mehrheit der Fälle indikativisch formuliert und können entsprechend den Bezug auf - aus der Perspektive des Fragenden - Vergangenes, Gegenwärtiges und Zukünftiges durch das gewählte Tempus zum Ausdruck bringen.

\subsection{Indikativ Futur im Römerbrief}

Vor diesem Hintergrund können nun auch die Futurformen im Römerbrief (siehe auch oben in Aussagesätzen, Kapitel 13, Abschnitt 2) erneut betrachtet werden. Eine beachtliche Anzahl dieser Verbformen entfällt in der Tat auf Fragesätze. Das erste Vorkommnis ist Röm 2,3, wo die Futurform jedoch Teil des ötı-Satzes ist,

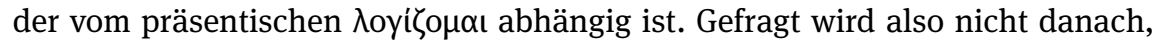
ob die angesprochene Person dem Gericht entkommen wird, sondern ob sie dies „denkt.“35

In Röm 2,26 begegnet die Frage „Wird nicht seine Unbeschnittenheit als

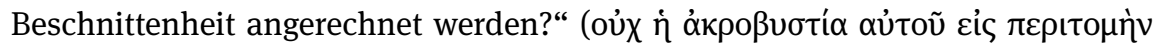
$\lambda$ jedoch noch von der Erfüllung des vorgeschalteten prospektiven Konditionalsatzes abhängig. ${ }^{36}$

Röm 3,3 ist ein ganz ähnlicher Fall, jedoch mit zwei Unterschieden: Die Protasis ist indefinit und die Frage in der Apodosis lässt hier eine verneinende Antwort erwarten: „Wird etwa die Untreue die Treue Gottes aufheben?“ ( $\mu \grave{\eta} \dot{\eta} \alpha \dot{\pi} \iota \sigma \tau i ́ \alpha$

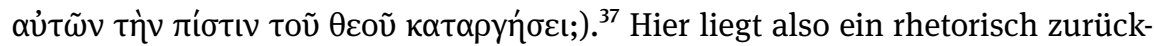
haltendes „prädiktives Diserzählen“ vor.

Vgl. demgegenüber die Beispiele bei Schwyzer-Debrunner II 703.

34 Vgl. GGNT 269a. Dort wird die rhetorische Frage als dritte Kategorie neben Entscheidungsfrage und Ergänzungsfrage eingeführt, da sich die erwartete Antwort jeweils unterscheidet. Auch wenn im Fall der rhetorischen Frage allerdings keine Antwort vom Gegenüber erwartet wird, ist dort eine Antwort vorausgesetzt. Für die Praxis ist es daher sinnvoller, zwischen Entscheidungsund Ergänzungsfragen zu unterscheiden, die mit einer unterschiedlich starken Aufforderung zum expliziten Beantworten verbunden sind. Vgl. fürs Deutsche Duden 1395, wo der Komplex der „rhetorischen“ Fragen in Aufforderungssätze („Kannst Du mir mal das Salz reichen?“) und Ausrufesätzen („Habe ich nicht schon oft genug auf die Unmöglichkeit der Situation hingewiesen?") unterteilt wird.

35 Siehe zu Röm 2,3 unten, Abschnitt 3.3.5.

36 Siehe zum Konditionalgefüge in Röm 2,26 unten, Abschnitt 5.2.2.2.

$37 \mathrm{Zu}$ den indefiniten Konditionalsätzen im Römerbrief siehe unten, Abschnitt 5.2.1. 
Die Konstruktion mit $\mu$ ń (ohne Konditionalsatz) liegt auch in Röm 9,20 vor: ${ }^{38}$ „Wird etwa das Geformte dem Former sagen: ,Was hast Du mich so gemacht?““ ( $\mu$ ì

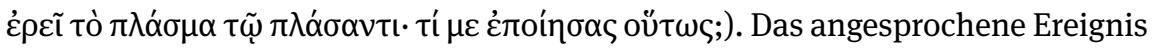
ist hier also selbst wiederum die Äußerung einer Frage. ${ }^{39}$

Auch drei Ergänzungsfragen werden im Römerbrief mit Indikativ des Futurs formuliert, bei denen das Interrogativpronomen tís ist. Die darauf von Paulus erwartete Antwort fällt jedoch ganz unterschiedlich aus. Abhängig von der für Röm 7 angenommenen Auslegungstradition, wird die Rekonstruktion der von Paulus erwarteten Antwort auf die verzweifelte Frage „Wer wird mich retten aus

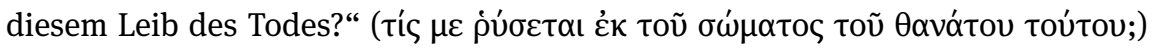
in 7,24 ganz unterschiedlich ausfallen: Entweder, man nimmt an, dass die Danksagung in 7,25 selbst direkt die Antwort liefert, oder aber man argumentiert, dass „aus dem immanenten Horizont“ von Röm 7,7-23 folge, die Antwort müsse „Niemand!“ lauten. ${ }^{40}$ Damit wäre dann freilich - innerhalb der erzählten Welt auch das Geschehen einer zukünftigen Befreiung insgesamt diserzählt.

Ein solches prädiktives Diserzählen liegt wohl auch in Röm 8,33 vor: „Wer

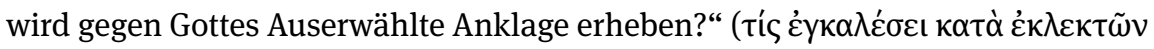

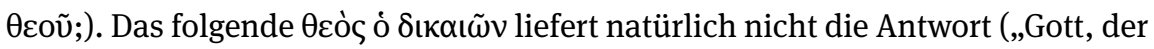
Richtende“), sondern zeigt auf, dass die Antwort „Niemand!“ lauten muss. Der Teilvers ist also als Nominalsatz zu lesen: „Gott [ist ${ }^{41}$ derjenige, der rechtfertigt“

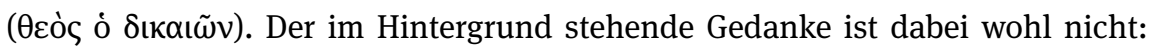

38 In Röm 3,3 und 9,20 folgt auf $\mu$ '́ der Ind. Fut. Nun kann im NT mit diesem Tempus teilweise auch die Funktion des deliberativen Konjunktivs übernommen werden (GGNT 269f), sodass die Bedeutung des Satzes in einem solchen Fall die eines deliberativen Fragesatzes ist. Während in 9,20 eine solche Auslegung nahe liegt und in 3,3 zumindest zu erwägen ist, ist damit freilich nicht gesagt, dass $\mu$ ń Ind. Fut. automatisch auf eine deliberative Frage verweist. Vgl. Mt 7,9 (... $\mu \grave{\eta}$

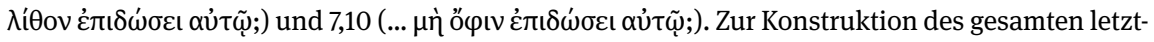
genannten Verses siehe GGNT 269d. Zur standardgriechischen Konstruktion siehe BDR $469^{1}$ und

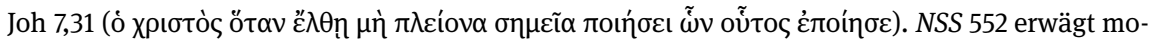
dalen Gebrauch. Der zwischengeschaltete temporale Nebensatz passt jedoch gut zur Annahme eines reinen Zukunftsbezugs, insbesondere, da der Vergleich ja auch nicht nur auf das Vermögen Jesu abzielt, sondern seine tatsächlichen Taten benennt.

39 Zum vermutlich modalen Gebrauch des Futurs siehe unten, Abschnitt 3.1.

40 Wilckens, Brief II, 95. Wolter, Brief I, 462-463 sieht in V. 25 eine Rückkehr vom „Ich“ zu Paulus. Entsprechend betont er, dass die Frage in V. 24 nicht eine „, rhetorische Frage, deren Antwort Paulus schon kennt,“ sei. Als Teil des vom Ich entworfenen Bildes habe sie „rein illustrativen Charakter und verlangt darum nach keiner Antwort.“ Innerhalb des „Bildes“ gilt dies aber doch wohl schon.

41 Gott hat also die syntaktische Subjektrolle inne. Die auf http://www.opentext.org/texts/NT/ Rom/view/clause-ch8.v0.html gebotene Analyse scheint von der Informationsstruktur her die Grammatik falsch zu deuten. 
„Wen Gott nicht verurteilt, sondern rechtfertigt, den kann auch kein anderer verurteilen. “42 Denn dann wäre wohl die Subjektergänzung als rhematisch aufzufassen: „Gott ist (nicht der Ankläger, sondern) der/ein Rechtfertige/r.“ Vielmehr ist der Gedanke wohl, dass die einmal erfolgte Rechtfertigung durch Gott $(8,30)$, weil sie eine Rechtfertigung Gottes ist (das Subjekt ist also rhematisch), keine spätere Veränderung des Urteils mehr zulässt, sodass auch keine (rechtskräftige) Anklage mehr vorgebracht werden kann, von niemandem.

Nach demselben Muster ist auch die direkt im Anschluss formulierte Frage in 8,34 zu verstehen, wobei hier (wenn die Akzentuierung übernommen wird) das bei Paulus sehr seltene futurische Partizip zum Einsatz kommt: „Wer (ist derje-

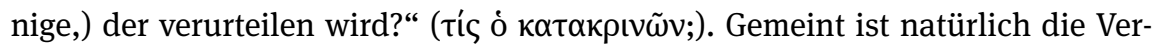
urteilung der in V. 33 genannten Auserwählten und abermals muss die Antwort „Niemand!“ lauten. Denn: „Christus [ist] derjenige, der gestorben, ja mehr noch,

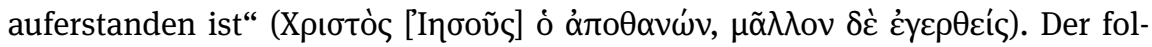
gende Relativsatz stellt vermutlich eine konsekutive Verbindung her: als Aufer-

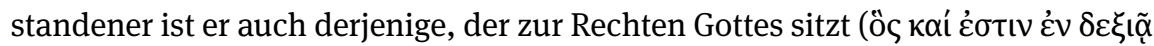

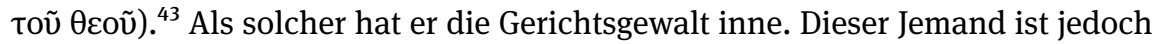

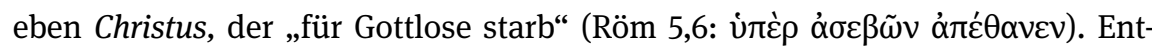
sprechend folgt in einem weiteren Relativsatz nun auch keine richtende Funktion, sondern, durch den Fokus seines „für uns“ (ن் Verhaltens, seine Rolle an der Seite Gottes: sein Eintreten zu unseren Gunsten (ös

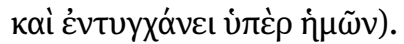

Auch auf die Frage in 8,35, die an das Verhältnis zu Christus anknüpft - „Wer

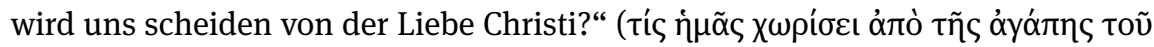
Xpıбтoṽ;) - muss letztlich, trotz Erwägung aller möglichen Kandidaten im selben Vers, die Antwort „Niemand!“ lauten (vgl. 8,38-39).44

Auch Ergänzungsfragen mit Tí und Indikativ Futur begegnen im Römerbrief, jedoch ausschließlich in der Kombination mit غ́poṽ $\mu \varepsilon v$ (Röm 3,5, 4,1, 6,1, 7,7, 8,31, 9,14, 9,30). In einigen Fällen folgt eine explizierende Anschlussfrage (mit $\mu \eta$ in Röm 3,5 und 9,14; Entscheidungsfrage ohne Partikel in 7,7, Ergänzungsfrage mit Tís und Bedingungssatz in 8,31). Die Nähe des Futurgebrauchs zum deliberativen Konjunktiv ${ }^{45}$ wird daran deutlich, dass in Röm 6,1, der einzigen Stelle, ${ }^{46}$ wo der

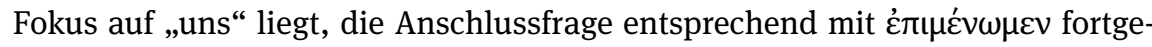
führt wird (d.h. „Sollen wir bleiben ...?“).

42 Wolter, Brief I, 545.

43 Vgl. GGNT 290e zum Relativsatz mit qualitativ-konsekutivem Nebensinn.

44 Siehe zu dieser Passage unten, Abschnitt 3.2.2.

45 Vgl. BDR 366,2.

46 Sehr umstritten ist natürlich Röm 4,1. 
In Röm 6,2 und 8,32 taucht zudem die Einleitung der Frage mit $\pi \tilde{\omega} \varsigma$ auf: „Wie

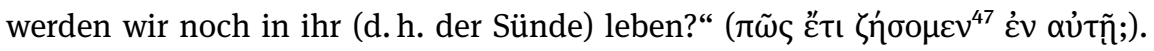

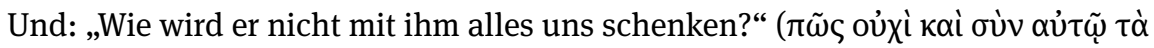
$\pi \alpha \dot{v} \tau \tau \alpha \dot{\eta} \mu \tilde{v} \nu \chi \alpha \rho i ́ \sigma \varepsilon \tau \alpha$; ). In beiden Fällen ist die von Paulus implizierte Antwort auf die Frage nach der Art und Weise mit welcher der Verbinhalt verwirklicht werden wird/könnte: „Keinesfalls!“48 In beiden Fällen wird die Frage durch einen Relativsatz vorbereitet, der einen Kontrast zum in der Frage erwogenen Geschehen

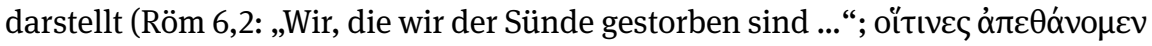

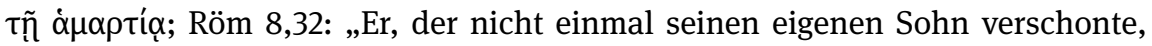

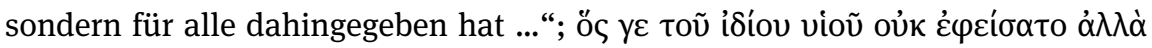

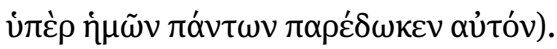

\subsection{Deliberativer Konjunktiv}

Es wurde bereits auf die konjunktivische Anschlussfrage in Röm 6,1 verwiesen. Ist eine Frage im deliberativen Konjunktiv formuliert ${ }^{49}$ - dies ist sowohl bei Entscheidungs- als auch bei Ergänzungsfragen möglich, unabhängig davon, ob sie lediglich rhetorischer Natur sind oder nicht ${ }^{50}$ - so liegt damit (a) Zukunftsbezug vor und dieser ist (b) als unsicher markiert. ${ }^{51}$ So ist vermutlich ${ }^{52}$ Röm 3,8, entsprechend der darin zitierten üblen Nachrede mit adhortativem Konjunktiv ( $\pi$ oเń $\sigma \omega \mu \varepsilon \nu=$,Lasst

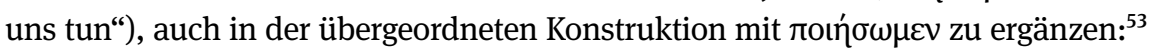

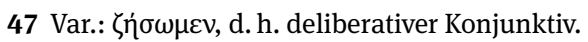

48 Vgl. BDAG 6457,1ayo.

$49 \mathrm{Zu}$ diesem siehe GGNT 210d. Zum historischen Zusammenhang mit dem adhortativen Konjunktiv siehe Schwyzer-Debrunner II 318. Für die Möglichkeit, dass der Indikativ des Futurs den Konjunktiv im deliberativen Fragesatz ersetzt, siehe oben, Abschnitt 2.2.

50 GGNT 269b. Entscheidungs-, Ergänzungs- und rhetorische Fragen können allesamt als deliberative Frage vorliegen.

51 Siehe GGNT 210 fur deliberativen Frage.

52 Für andere Auflösungen siehe BDR 427.

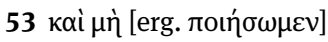

$\kappa \alpha \theta \dot{\omega} \varsigma \beta \lambda \alpha \sigma \varphi \eta \mu о u ́ \mu \varepsilon \theta \alpha$

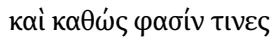

$\dot{\eta} \mu \tilde{\alpha} \varsigma \lambda \varepsilon_{\varepsilon} \varepsilon \mathrm{I} v$

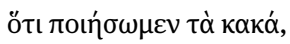

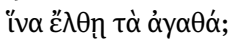

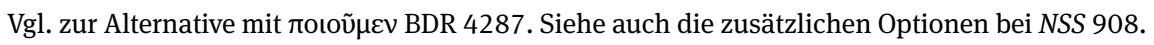


„Und sollen wir nicht ${ }^{54}$ so handeln, wie wir verlästert werden und wie einige behaupten, dass wir sagen: ,Lasst uns das Böse tun, damit das Gute komme!'?“ Derselbe Gedanke - nicht mit finalem Vorverweis sondern Blick auf den begründenden Status - ist in der Frage Röm 6,15 formuliert: Auf „Was nun?“ (Tí oũv;) folgt: „Sollen wir sündigen, weil wir nicht mehr unter Gesetz, sondern unter Gnade sind?“

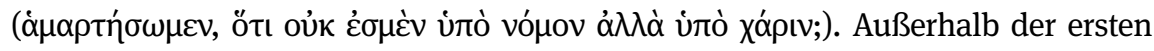
Person erscheint der deliberative Konjunktiv in der Reihung von Fragen in Röm 10,14-15a. ${ }^{55}$ Die genauere Analyse zeigt aber, dass diese Passage unter dem Gesichtspunkt des Diserzählens vergangener Ereignisse zu besprechen ist. ${ }^{56}$

\subsection{Auswertung}

Zusammenfassend lässt sich sagen: Fragesätze mit Indikativ Futur oder deliberativem Konjunktiv bieten ein äußerst vielseitiges Instrumentarium, um zukunftsungewisse Vorausdeutungen vorzunehmen.

Durch die Partikel kann etwa der Grad der Sicherheit des potenziellen Ereignisses als wahrscheinlich oder unwahrscheinlich dargestellt werden, wodurch bestimmte Handlungsfortsetzungen „potenziell erzählt“ bzw. „graduell diserzählt" werden.

Auch durch rhetorische Ergänzungsfragen (z. B.: „Wer wird ...?“) kann ein zukünftiger Handlungsstrang als unwahrscheinlich bis kaum möglich dargestellt werden, wenn darauf keine zufriedenstellende Antwort naheliegt.

Insgesamt bieten Ergänzungsfragen die Gelegenheit, die Hörer/Leser in die Ausgestaltung der futurischen Handlung miteinzubeziehen, indem etwa ein Ereignis, ein Agens, ein Motiv, oder ein anderer (z. B. lokaler oder temporaler) Umstand erfragt wird. Paulus macht von all diesen verschiedenartigen zukunftsungewissen Vorausdeutungen Gebrauch. Gerade im Vergleich mit der relativ geringen Anzahl solcher Futurformen im Römerbrief, welche der uneingeschränkten Ankündigung zukünftiger Ereignisse dienen, fällt diese ausführliche Ausgestaltung lediglich potenzieller zukünftiger Ereignisfolgen besonders auf.

54 Die Übersetzung mit „etwa“ (NSS 908) setzt wohl ein Verständnis als Fragepartikel voraus. Allerdings ist $\mu$ '́ allein schon wegen des Modus gesetzt (vgl. GGNT 210d).

55 Vgl. BDR 366,1 (,... zuweilen [hat den Konj.] auch die 2. und 3. Ps., und zwar so, daß die Bedeutung mehr eine futurische oder potentiale wird“). Für Beispiele siehe BDR $366^{4}$.

56 Siehe daher Kapitel 12, Abschnitt 3.2. zu dieser Passage. 


\section{Aussagesätze}

\subsection{Modales Futur}

\subsubsection{Hinführung}

Aussagesätze über die Zukunft können auf verschiedene Weise abgeschwächt werden, sodass auch mit ihnen zukunftsungewisse Vorausdeutungen vorgenommen werden können. Der wichtigste Aspekt, der in diesem Kontext zu berücksichtigen ist, ist das Futur selbst, welches mit modaler Sinnrichtung nicht die absolute Überzeugung über ein Eintreffen zukünftigen Geschehens aussagt.

Ob das Futur überhaupt in erster Linie ein Tempus darstellt, ist durchaus umstritten, da es sich von anderen Zeiten durch die Tatsache unterscheidet, „that reference to future time is inherently related to events as yet unrealized and therefore unknown. “57 Auch wenn entsprechend immer wieder dafür argumentiert wird, das Futur nicht den Tempora, sondern dem Bereich der Modalität zuzuordnen, muss aber doch festgehalten werden, dass Sprecher mit derselben Bestimmtheit über zukünftige Ereignisse sprechen können wie über vergangene oder gegenwärtige..$^{58}$

Unumstritten ist aber auf jeden Fall auch, dass futurische Verbformen häufig in Kontexten verwendet werden, in denen (lediglich) eine Absicht angesprochen wird. ${ }^{59}$ Die Aussage bezieht sich natürlich weiterhin auf ein zukünftiges Ereignis, die Bestimmtheit, mit welcher das Geschehen vertreten wird, ist jedoch abhängig von den Bedingungen, unter welchen diese Absicht erst noch verwirklicht werden muss. ${ }^{60}$ Eine solche ,voluntative“ Bedeutung kann natürlich primär für Absichten verwendet werden, welche eigene Vorhaben betreffen, kann jedoch auch außerhalb der 1 . Person ${ }^{61}$ verwendet werden, wenn etwa einer dritten Partei eine Absicht zugesprochen oder von einem Gegenüber erfragt wird. ${ }^{62}$ In der direkten Anrede einer anderen Person kann dieser der eigene Wille auch ,aufgezwungen“

57 Makropoulos, Future, 8.

58 Vgl. etwa Makropoulos, Future, 8-9.

59 Im sigmatischen Futur ist dies auch sprachgeschichtlich im (teilweise) zugrundeliegenden Desiderativ zu erkennen. Vgl. Schwyzer-Debrunner I 787.

60 CGCG 33.43 schreiben, "the future indicative presents the realization of some action in the future as (virtually) certain.“ Sie führen an ,various communicative purposes“ aber auch „predictions, statements of intentions, ... suggestions“ an. Letztlich bestimmen sie die Gewissheit relativ: „... as (virtually) certain (more so than, for example, the ,potential‘ optative ...).“

61 Der voluntative Gebrauch ist natürlich nicht auf die 1. Person Singular beschränkt. Vgl. toútoıৎ

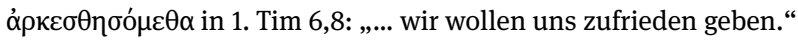

62 Schwyzer-Debrunner II 290: „er will kommen“ und „willst du kommen?“ 
werden, ${ }^{63}$ sodass dann imperativischer Gebrauch vorliegt. Neben der Nuance des Wollens und Sollens tritt im NT eventuell auch unter semitischem Einfluss des Imperfekts eine Fokussierung auf das Können auf. ${ }^{64}$

Für die Beschäftigung mit paulinischen Protonarrativen ist die Kategorie des modalen Gebrauchs des Indikativs des Futurs insofern von Bedeutung, da gerade bei Äußerungen in der 1. Person oft nicht klar gesagt werden kann, ob hier tatsächlich ein „rein futurischer Gebrauch für das, was sein oder geschehen wird“65 vorliegt, oder ob bei der Äußerung der Absicht, vorausgesetzt ist, dass sie nur unter bestimmten Umständen auch verwirklicht werden wird. Die Übergänge sind fließend, ${ }^{66}$ und in wörtlichen Übersetzungen mit dem Futur I im Deutschen fällt die Problematik meist nicht weiter auf, da das Deutsche über eine ganz ähnliche Bandbreite modaler Gebrauchsweisen verfügt. ${ }^{67}$ Wenn Paulus etwa in Röm 9,19 und 11,19 ع́рعĩ ( $\mu$ o ) oũv formuliert, so kann dies im Sinne einer echten Ankündigung verstanden werden, die in einen fiktiven Dialog eingebunden ist (im Sinne von: „Als nächstes wirst Du darauf erwidern: ...“). ${ }^{68}$ Die Aussage des Paulus lässt sich allerdings ebenso gut als dem Wesen nach lediglich eine Vermutung über einen nun möglichen Einwand verstehen. ${ }^{69}$ Dann läge hier ein prospektiver Gebrauch des Futurs vor: „Angesichts dessen, was ich gerade geschrieben habe, ${ }^{70}$ wirst Du vielleicht die folgende Frage haben: ... “ Eine solche Äußerungsabsicht kann im Text durch Signale nahegelegt werden. So führt wohl

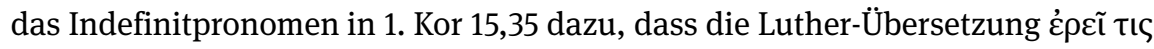
als „Es könnte aber jemand fragen: ...“ übersetzt, während es in Röm 9,19/11,19 beide Male „Nun sagst/sprichst Du“ heißt. ${ }^{71}$

63 Schwyzer-Debrunner II 290: „A: ,Ich will nicht,‘ B: ,Du willst‘ (,hast zu wollen, mußt wollen')."

64 Vgl. GGNT 202b. Zur Möglichkeit eines ebenfalls semitisch beeinflussten zeitlosen Futurs siehe GGNT 202j.

65 Vgl. zur Kategorie Schwyzer-Debrunner II 292.

66 GGNT 202b.

67 Vgl. Duden 729.

68 Siehe etwa zu Röm 9,19 Wilckens, Brief II, 201, der ohne Einschränkung formuliert: „Hier interveniert der Gegner wieder und spitzt seinen Einwand nun vom Menschen aus zu.“

69 Vergleichbar ist in mancher Hinsicht Platon, Resp. 338c, angeführt bei CGCG 33.45.

$70 \mathrm{Vgl}$. Schwyzer-Debrunner II 290. Anders als beim voluntativen/imperativischen Gebrauch ist es hier nicht ein Wille, sondern sind es äußere Umstände, welche das Eintreten eines bestimmten Ereignisses vermuten lassen. Die Kategorie wird zuweilen verwendet, um ein nicht-temporales, „logisches“ Futur zu begründen, eignet sich dafür aber keinesfalls. Vgl. Abschnitt 3.2.

71 Interessanterweise nimmt die NGÜ eine explizite Abschwächung nicht in 1. Kor 15,35 vor, aber dafür in Röm 11,19: „,Aber,‘ entgegnest Du vielleicht, ,damit ich eingepfropft werden konnte, sind andere Zweige ausgebrochen worden!““ 
Dass das Verständnis des genau Gemeinten auch im Griechischen für Muttersprachler nicht immer eindeutig ist, wird interessanterweise gerade auch durch ein Beispiel einer missglückten Kommunikation bei Paulus belegt: In 2. Kor 1,18-22 ist Paulus gezwungen, durch einen theologischen Exkurs die Eindeutigkeit seines „Wortes“ zu verteidigen, weil er den Reiseplan 2. Kor 2,15-16 nicht in die Tat umgesetzt hat. Entsprechend lautet der Vorwurf an ihn, sein „Ja“ sei zugleich auch ein „Nein,“72 d. h., seine Ankündigung, die Korinther (doppelt) zu besuchen sei letztlich nicht viel Wert gewesen, da Paulus seine Ankündigungen

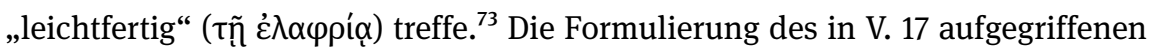
Vorwurfs legt aber die Annahme nahe, dass das von den Korinthern wahrgenommene Problem nicht so sehr die (erneute) Unfähigkeit des Paulus, an seinen Plänen festzuhalten, ist, sondern der Kontrast zwischen der eindeutigen Ankündigung (,Jaja“) und seiner späteren Entscheidung, sich doch anders zu verhalten („Neinnein“). Die Argumentation setzt voraus, dass die Korinther den in 2. Kor 1,14-16 entworfenen Plan nicht erst durch den Brief erfahren, ${ }^{74}$ ihn also wohl bereits vom Zwischenbesuch oder Tränenbrief ${ }^{75}$ her kennen. ${ }^{76}$ Seinen ursprünglichen Wortlaut kennen wir daher leider nicht, da im 1. Korintherbrief lediglich eine frühere Version des Vorhabens überliefert ist (1. Kor 16,5-8). Doch ist davon auszugehen, dass hier nicht zuletzt auch das Problem im Hintergrund steht, dass die einen Besuch ankündigenden Futurformen von Verben selbst nicht anzeigen, mit welcher Sicherheit der Sprecher davon ausgeht, dass die angesprochen Ereignisse auch eintreten werden. Interessanterweise nimmt Paulus im uns

72 Diese Auslegung setzt voraus, dass V. 17 einen Vorwurf an Paulus aufgreift. Für die Syntax bedeutet dies, dass tò vaì vaì Subjekt ist und kaì tò oủ oủ die Ergänzung darstellt: ,,... sodass das Jaja‘ (zugleich) auch ein ,Neinnein“ wäre.“ Alternativ wird teilweise argumentiert, jeweils das zweite voí und oủ stellten die Subjektidentifikationsergänzung zum jeweils ersten Vorkommnis dar. Das hieße dann: ,... sodass das ,Ja‘ ein ,Ja‘ und das ,Nein‘ ein ,Nein‘ wäre.“ Vgl. Young, „Note,“ 407. Dann wäre V. 17 kein aufgegriffener Vorwurf, sondern ein Argument des Paulus

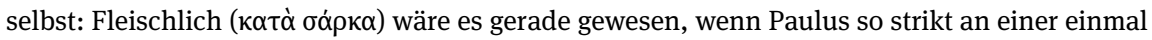
gegebenen Zusage festgehalten hätte.

73 Vgl. Heilig, Triumph, 156-161.

74 So richtig herausgearbeitet von Schmeller, Brief I, 102.

75 Der in 2. Kor 13,2 genannte schonungslose Besuch ist vermutlich der erste Teil des Doppelbesuchs aus 2. Kor 1,15. Dieser Doppelbesuch wird den Korinthern wohl im Rahmen des Zwischenbesuchs angekündigt worden sein. Erst nach dessen abrupten Ende entschließt sich Paulus aus Rücksicht, doch anders vorzugehen (2. Kor 1,23-2,22). Der stattdessen verfasste Tränenbrief wird die Korinther wohl dann auch über das Aussetzen des Planes des Doppelbesuches in Kenntnis gesetzt haben (vgl. Schmeller, Brief II, 103).

76 Die gesamte Rekonstruktion ist natürlich im höchsten Maße abhängig von den zahlreichen Einzelentscheidungen die im Hinblick auf Ablauf der korinthischen Korrespondenz getroffen werden. Die übersichtlichste Diskussion der Optionen bietet Schmeller, Brief I, 92-103. 
vorliegenden Reiseplan 1. Kor 16,5-8 durchaus eine sorgfältige Modalisierung durch verschiedene sprachlichen Mittel vor (vgl. unten, Abschnitt 3.2.2).

\subsubsection{Modales Futur in den Paulusbriefen}

Im Römerbrief ist der modale Gebrauch des Futurs vor allem mit der Frage „Was sollen wir (nun) sagen?“ ( $\tau$ í [oũv] غ́poũ $\mu \varepsilon v$ [...];) verbunden (Röm 3,5, 4,1, 6,1, 7,7, 8,31, 9,14, 9,30). Nennenswert ist jedoch auch Röm 3,6, wo das Futur offenbar den

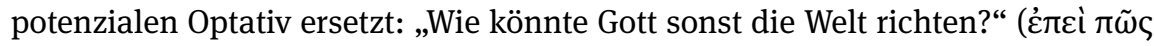

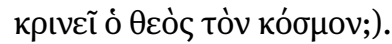

Äußerst bemerkenswert ist, dass der modale Gebrauch des Futurs in besonderer Häufung in der Korinther-Korrespondenz auftritt. So fragt Paulus in 1. Kor 6,5, ob es denn gar keinen Weisen unter den Korinthern gäbe, der zwischen

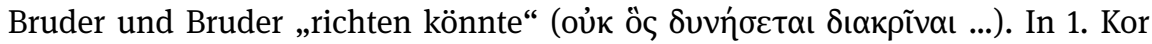
6,12 meint Paulus: „ich werde mich von nichts beherrschen lassen“ ( $\alpha \lambda \lambda$ ’ oủk

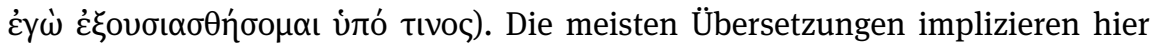
ein modales Verständnis (Elberfelder: „aber ich will mich von nichts beherrschen lassen“; Luther: „aber es soll mich nichts gefangen nehmen“), rechnen also explizit mit der Möglichkeit, dass das Ich sich doch beherrschen lassen könnte. ${ }^{77}$ Auch 1. Kor 9,15 wird wohl nicht als schlichte Ankündigung zu lesen

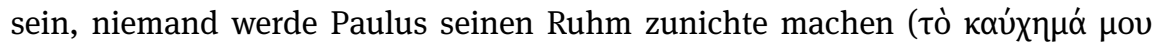

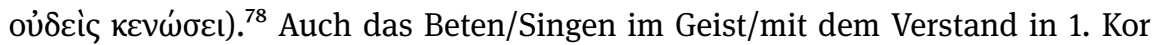

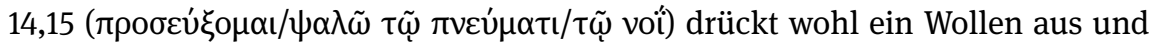
nicht eine schlichte Ankündigung. ${ }^{79}$ Das erklärt wohl auch den (lautlich identischen) Konjunktiv Aorist $\pi \rho \circ \sigma \varepsilon u ́ \xi \omega \mu \alpha$ เ für die erste oder beide Formen des

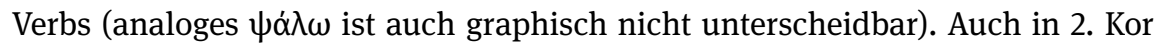
10-13 kommen einige Verbformen in Betracht, insbesondere die Aussagen zum „Rühmen werden/wollen“ in 2. Kor 10,13, 11,18, 12,5 (2x) und 12,9. ${ }^{80}$ In 2. Kor 12,1

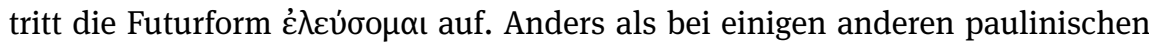

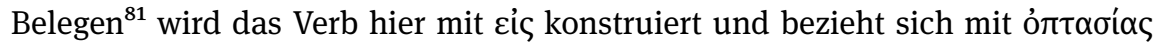

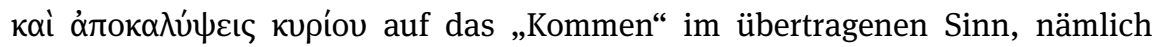

77 Schrage, Brief II, 19 kommentiert das Tempus nicht, sagt aber Paulus „warne“ hier vor etwas. 78 Deswegen muss man - immerhin außerhalb der 2. Person - natürlich noch lange nicht (Schrage, Brief II, 321) einen imperativischen Gebrauch (vgl. GGNT 202f) postulieren, da auch allgemeiner ein Sollen zum Bedeutungsspektrum des modalen Futurs gehört (GGNT 202d).

79 Vgl. GGNT 202c.

$80 \mathrm{Zu}$ 2. Kor 12,5 und der Evidenz im Kontext vgl. aber auch unten, Abschnitt 5.2.2.3 und bereits Kapitel 8, Abschnitt 5.2.2.1.

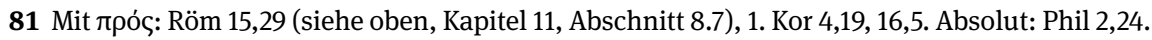
In Röm 9,9 erscheint es innerhalb eines Zitats (siehe oben, Kapitel 13, Abschnitt 2.2.2). 
auf die Thematisierung der Erscheinungen und Offenbarungen des Herrn im Diskurs. Ebenfalls von Interesse ist die Aussage in 2. Kor 11,9, wonach Paulus sich in allem so „gehalten“ habe, dass er den Korinthern nicht zur Last gefallen wäre

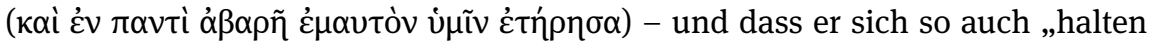

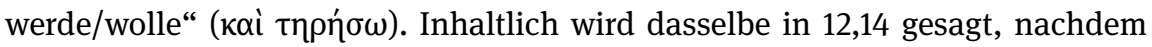
Paulus anmerkt, dass er „bereit“ für einen weiteren, den dritten, Besuch wäre

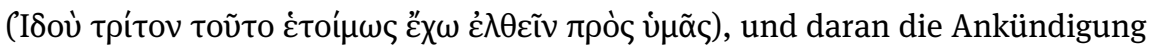

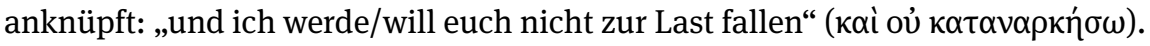

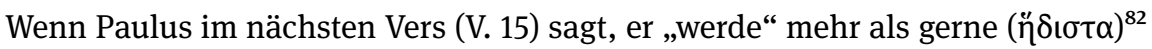

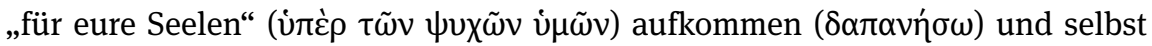

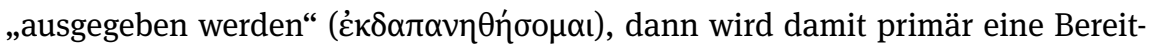
schaft ausgedrückt.

Im Rest des corpus Paulinum erscheint ein modaler Gebrauch des Futurs insgesamt seltener, tritt aber doch an manchen Stellen auf. $\mathrm{Zu}$ diskutieren wäre etwa das „Auslöschen“ ( $\pi \varepsilon \pi \nu \rho \omega \mu \varepsilon ́ v \alpha)$ der Pfeile des Bösen in Eph 6,16 im Sinne lediglich eines Vermögens. In Phil 4,4 folgt auf die Aufforderung zum allzeitigen

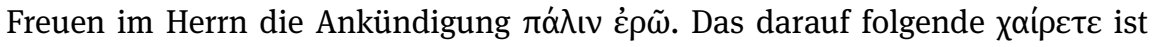
sowohl Inhalt als auch Implementierung dieser Ankündigung. Gerade im Vergleich zum präsentischen $\pi \alpha \dot{\lambda} \iota v$ $\lambda \dot{\gamma} \gamma \omega$ in Gal 1,9 scheint das Futur hier einen Schwerpunkt auf den Willen zu legen. ${ }^{83}$

\subsection{Adverbiale Abschwächung}

\subsubsection{Hinführung}

Es wurde bereits auf 1 . Kor 15,35 verwiesen und darauf aufmerksam gemacht, dass hier das Indefinitpronomen ein Verständnis des Futurs im Sinn einer bloßen Möglichkeit unterstützt, also analog zum (bei Paulus nicht belegten) potenzialen Optativ. ${ }^{84}$ Dies ist nur eines von vielen Beispielen dafür, wie auch lexikalisch eine Ankündigung im Futur explizit abgeschwächt werden kann - dies schließt auch den Gebrauch von Adverbien ein, ${ }^{85}$ wobei diese nicht isoliert zu betrachten sind, sondern im Zusammenhang mit anderen Textsignalen zu verstehen sind.

82 Vgl. ர் $\delta \dot{c} \omega \varsigma$ für das so bereitwillige Ertragen der Toren in 2. Kor 11,19.

$83 \mathrm{Zu} \pi \alpha \dot{\lambda} \iota \mathrm{v} \lambda \varepsilon \dot{y} \omega$ vgl. auch 2. Kor 11,16.

84 Zur Konstruktion von tıc mit der 2. Person Signular siehe GGNT 255f. Zum potenzialen Optativ allgemein siehe GGNT 211d. Siehe CGCG 34.13 für den klassischen Gebrauch „to describe actions that might hypothetically occur, or to make a weak assertion.“

85 Siehe Dik, „Degree Adverbs“ für das klassische Griechisch. 


\subsubsection{Der Reiseplan in 1. Kor 16,2-8}

Es lohnt sich, vor diesem Hintergrund den oben bereits angesprochenen, in 1 . Kor 16,5-8 vorgestellte Reiseplan (einschließlich der Verse 2-4) näher zu betrachten, da man an dieser Passage erkennen kann, wie ein modales Verständnis des Indikativs des Futurs mal mehr und mal weniger durch ergänzende Textsignale unterstützt wird.

Das Kommen des Paulus wird zunächst lediglich in zwei temporalen Neben-

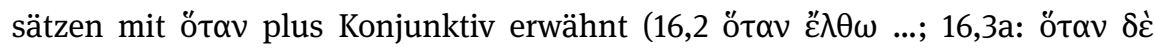
$\pi \alpha \rho \alpha \varepsilon^{\prime} v \omega \mu \alpha \iota$...). Das Senden von bewährten Mittelsmännern wird dann (16,3b) allerdings futurisch formuliert $(\pi \varepsilon \dot{\varepsilon} \mu \omega)$, während der Zweck dieser Sendung

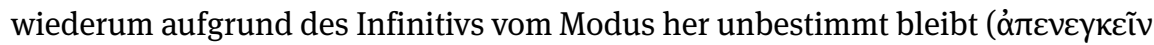

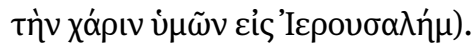

Im nächsten Vers $(16,4 b)$ qualifiziert Paulus deren Reise durch den Indikativ

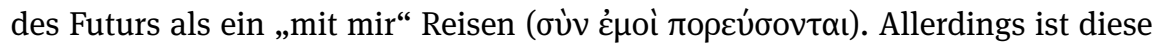
Ankündigung nun explizit von einer Bedingung abhängig gemacht (16,4a): „Wenn

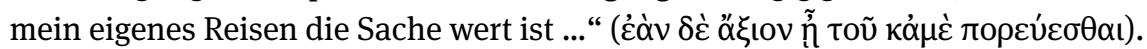

Eine indikativische Ankündigung des Kommens „zu euch“ findet sich dann

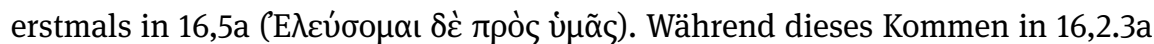
den temporalen Bezugspunkt für andere Ereignisse darstellte, ist es hier wiederum selbst an eine vorangehende Episode geknüpft $(16,5 b)$ : „... wenn ich Maze-

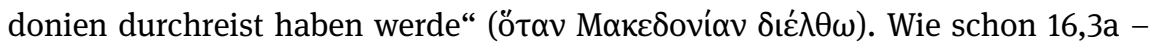
aber eben gerade nicht wie 16,4b - hat die Ankündigung des Besuchs in 16,5a also lediglich eine temporale Bedingung, deren Eintreffen im Text selbst nicht in Zweifel gezogen wird.

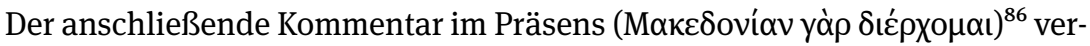
weist dabei nicht auf die sich bereits im Verlauf befindliche Durchreise, sondern betont deren vorübergehenden Charakter als Kontrastfolie für den Besuch in

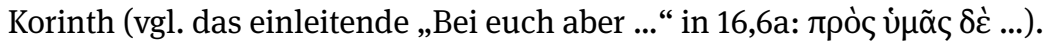

Interessant ist nun allerdings, dass Paulus zwar in 16,8 ebenfalls ohne

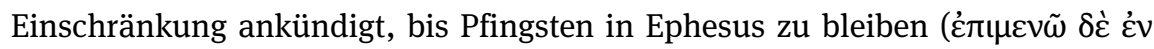

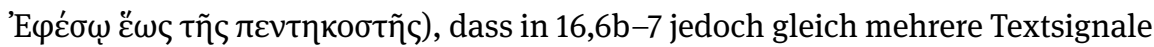
erscheinen, welche den Rezipienten helfen, den „Status“ der Ankündigung von 16,5a besser einzuordnen.

Das betrifft einerseits die Dauer des anvisierten Besuchs. Er soll nicht nur auf der Durchreise geschehen, wie dies für Mazedonien geplant ist (16,5b), ein Kurzbesuch wie er bei sofortiger Abreise auch jetzt schon möglich wäre (so 6,7a:

86 Siehe dazu Kapitel 13, Abschnitt 1. 


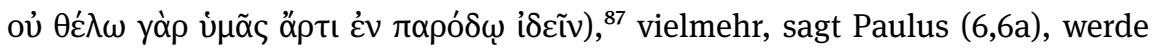

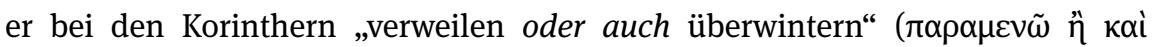

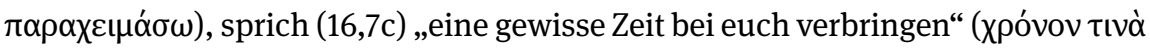

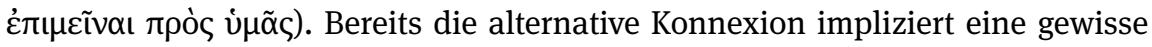
Unsicherheit. Der Bezug zur Wirklichkeit wird aber noch durch weitere Textelemente näher bestimmt: Die indikativischen Futurformen aus 6,6a werden einge-

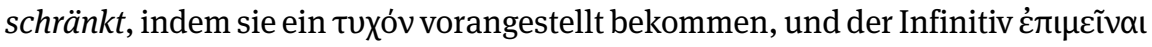
in 16,7c wird einerseits durch das einleitende Verb als „Hoffnung“ charakterisiert (16,7b: $\dot{\varepsilon} \lambda \pi i \zeta \omega)$ und ist andererseits von einem Konditionalsatz abhängig (16,7d):

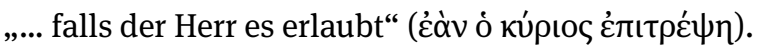

Auch wo keine explizite Einschränkung vorgenommen wird (das betrifft in diesem Text die drei Futurformen in 16,3b und 16,5a), ist natürlich nicht gesagt, dass im Umkehrschluss der Autor hier keinerlei Zweifel am Eintreten dieser Ereignisse hegt. Für die Analyse von Protonarrativen ist aber auf jeden Fall bedeutsam, festzustellen, wo durch zusätzliche explizite Einschränkungen Handlungsfortsetzungen betont als nur potenziell markiert werden - wobei hier der zugemessene Grad an Wahrscheinlichkeit natürlich je nach Situation ganz unterschiedlich ausfallen kann.

\subsubsection{Weitere paulinische Vorkommnisse}

túxov wird von Louw-Nida als Marker der Möglichkeit eingeordnet ${ }^{88}$ und steht

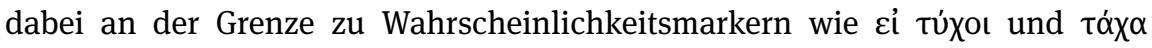
(„wahrscheinlich“) oder auch dem stärker abschwächenden l゙ $\sigma \omega \varsigma$ (,vielleicht“), ${ }^{89}$ welche jedoch bei Paulus selten sind und nicht in Verbindung mit zukünftigen Ereignissen auftreten.

Einen ganz ähnlichen Effekt hat jedoch $\mu$ ó $\lambda$ ı in Röm 5,7: Dass jemand für

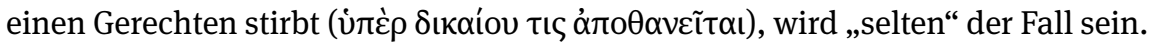
An die Stelle der Unwahrscheinlichkeit eines individuellen Ereignisses tritt hier eine statistische Aussage über ein Bündel an Ereignissen derselben Klasse. ${ }^{90}$

87 Siehe hierzu unten, Abschnitt 4.2.2.1.

88 LN 71.6.

89 LN 71.11-13.

90 Man kann hier - analog zum Präsens in der Erwägung des nächsten Satzes (7b: „für

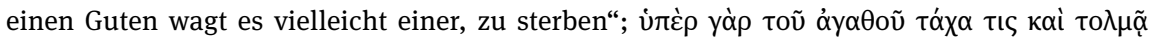

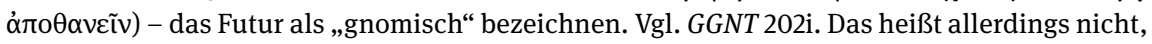
dass hier die futurische Referenz völlig aufgegeben wäre. Wallace, Grammar, 571 meint: „The idea is not that a particular event is in view, but that such events are true to life." Dies gilt freilich für sämtliche gnomischen Ausdrucksweisen. Dennoch gibt es keinen Grund zur Annahme, dass sich aoristische, präsentische und futurische Formen überhaupt nicht in der Per- 
Auch $\mu \eta \dot{~(+\mu \eta ́ \pi о \tau \varepsilon ~ e t c .) ~ k a n n ~ z u w e i l e n ~(i m ~ c o r p u s ~ P a u l i n u m ~ e v e n t u e l l ~ i n ~ 2 . ~ T i m ~}$

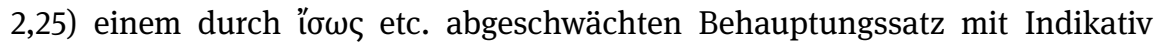
Futur nahekommen. ${ }^{91}$

\subsection{Abschwächung abhängiger Behauptungssätze durch epistemische Ausdrücke}

\subsubsection{Hinführung}

Durch adverbiale Modifikation lässt sich das in einem Hauptsatz angekündigte zukünftige Geschehen als mehr oder weniger sicher erwartet kennzeichnen. Dabei ist diese Einschätzung natürlich immer von der subjektiven Perspektive des Sprechers abhängig (beziehungsweise von der gewählten Fokalisierungsinstanz). Derselbe Effekt kann daher auch erreicht werden, indem eine Proposition als Gegenstand einer geistigen Einstellung beschrieben wird, welche ein differenziertes Maß an Überzeugung auszudrücken vermag. Das eine Ende des Spektrums bildet die Konstruktion mit oĩ $\delta \alpha / y ı v \omega \sigma \kappa \omega$, welche zukunftsgewisse Vorausdeutungen einleitet (siehe oben, Kapitel 13, Abschnitt 3.1).

Doch die Erwartung kann auch abgeschwächt werden, wenn der ötı-Satz von anderen epistemischen Ausdrücken abhängt, die nicht dasselbe Maß an Sicherheit implizieren. In diesem Kontext bedeutet „etwas erwarten,“ dass man „denkt, dass ein Ereignis geschehen wird.“92 Eine Bewertung des zukünftigen Geschehens als Hoffnung (also als sehnsuchtsvolles Erwarten) liegt dabei nicht vor. ${ }^{93}$ Dieser wertende Bezug auf Unsicheres in der Zukunft wird unten (Abschnitt 3.4) noch besprochen werden.

spektive auf die ins Auge gefasste Regelmäßigkeit unterscheiden. Vgl. deutsch: „Du wirst es kaum einmal erleben, dass ...“ Zur unterschiedlichen Konzeptualisierung von gnomischem Ind. Aor. im Vergleich zum Ind. Präs. vgl. oben, Kapitel 6, Abschnitt 3.4.2.4.

91 GGNT 267. Siehe unten, Abschnitt 3.5.2 zu Befürchtungssätzen.

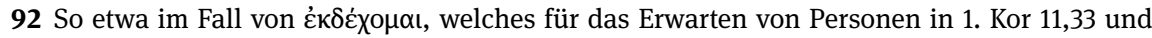

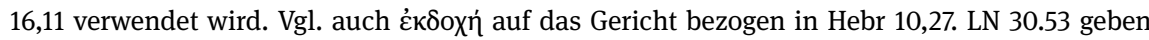
die Bedeutung als ,to expect something to happen“ an. Da „to expect“ ebenso wie „erwarten“ missverständlich ist, ist die von ihnen vorgeschlagene Entsprechung „to think that it will happen that" trotz ihrer Umständlichkeit für Übersetzungen zu erwägen.

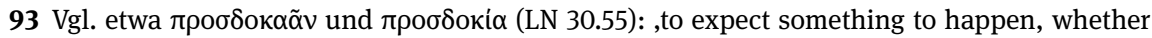
good or bad.' 


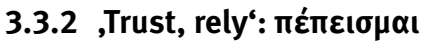

Ein wichtiges Verb, das in diesem Kontext berücksichtigt werden muss, ist $\pi \varepsilon i \theta \omega$. Das Verb hat im Perfekt Präsens-Bedeutung ${ }^{94}$ und die (1) starken Perfekt-Aktiv-Formen, $\pi \dot{\varepsilon} \pi$ o $\theta \alpha$ etc., haben den semantischen Inhalt ,to be so convinced that one puts confidence in someth. ${ }^{95}$ Die Rede vom „Vertrauen“ scheint allerdings bereits eine positive Einstellung vorauszusetzen. Wenn also durch ein AcI ein Geschehen vom Verb abhängig gemacht wird, so ist ein solcher Beleg am besten unter der Kategorie der Hoffnung zu besprechen (siehe dazu unten, Abschnitt 3.4). ${ }^{96}$

Dieses Problem scheint im (2) medial-passivischen Fall nicht zu bestehen. ${ }^{97}$ Dort ist dafür die Abgrenzung vom „Wissen“ nicht ganz einfach: Handelt es sich dabei überhaupt um eine Abschwächung, ${ }^{98}$ oder wird lediglich die Art und Weise betont, mit welcher ein Wissensstand erreicht wurde? ${ }^{99}$ Betrachtet man jedoch die Verwendungsweise von $\pi \varepsilon \dot{\pi} \varepsilon\llcorner\sigma \mu \alpha \iota$ in griechischen Texten Seite an Seite mit oĩ $\delta \alpha$, so fällt auf, dass die jeweils abhängigen Inhalte zwar mit derselben Sicherheit vertreten werden können, in der Regel durch die Zusammenstellung gerade der erstgenannte Teil als Meinung gekennzeichnet werden soll. ${ }^{100}$ Auch wird

94 GGNT 200b.

95 BDAG 5754,2. Zur intransitiven Bedeutung starker Perfekt-Aktiv-Formen bei einigen transi-

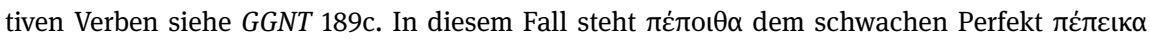
gegenüber, d.h., ,ich habe überredet.“

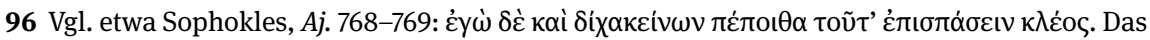
Erhoffte kann freilich, von einem anderen Standpunkt aus betrachtet, gräulichen Inhalt haben.

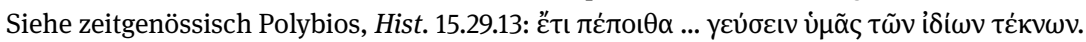

97 Vgl. Platon, Resp. 368a.

98 So wohl BrDAG 1602: ,to be convinced, give credence, believe.

99 Vgl. etwa LN 31.46: ,to come to believe the certainty of something on the basis of being convinced.

100 Vgl. etwa Galenos, Nat. fac. 1.1 zur Beobachtung, dass manche Leute im Hinblick auf Charakteristika von Pflanzen auf der einen Seite und Tieren auf der anderen Seite nicht von

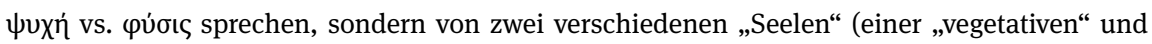

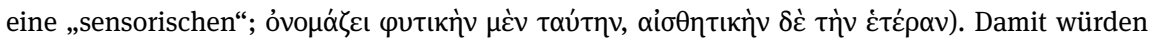
sie letztlich nichts anderes aussagen, sondern lediglich ungewöhnliche Wörter verwenden. Die

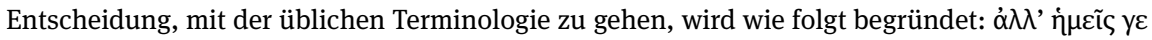

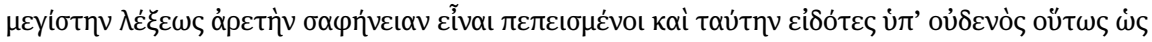

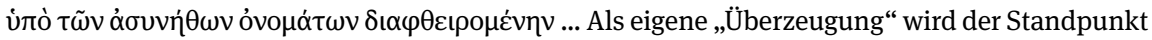
gekennzeichnet, dass Klarheit der höchste Wert der Sprache sei. „Gewusst“ wird hingegen, dass nichts dem abträglicher sei, als ungewöhnliche Ausdrücke. Der erste Teil des Arguments wird zwar ebenso vehement vertreten, wie der - wohl durch die Erfahrung gut gestützte - zweite Teil. Doch wird durch die Art der Formulierung gerade die Unterstellung ermöglicht, dass die „anderen“ die besagte Überzeugung nicht teilen. 
teilweise durchaus der Unterschied zwischen einer „bloßen“ Überzeugung und einem fundierteren Wissen markiert. ${ }^{101}$

Vor diesem Hintergrund ist die Koordination mit oĩo $\alpha$ in Röm 14,14 äußerst

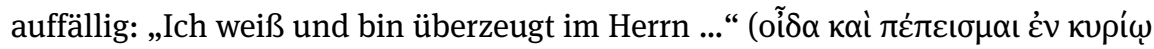
'Inбoṽ). Hier wird das Wissen letztlich dadurch aufgewertet - beziehungsweise in seinem Status als Wissen und nicht nur persönliche Meinung überhaupt erst bestätigt - dass es sich dabei um eine Überzeugung handelt, die auf den Herrn selbst ${ }^{102}$ zurückgeführt werden kann. ${ }^{103}$ Der Anspruch eines - etwa schöpfungstheologisch fest verankerten - vermeintlichen Wissens ist letztlich umstrittener, als der Verweis auf eine überlieferte Überzeugung, die jedoch am Herrn Jesus selbst festgemacht werden kann.

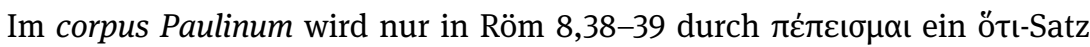
eingeleitet, welcher Zukunftsbezug hat. Dabei ist der eschatologische Kontext klar durch 8,30.33 markiert. Die Einführung von Elementen des irdischen Lebens (Bedrängnis, Angst, Verfolgung, Hungersnot, Blöße, Gefahr, Schwert) in V. 35 ändert an dieser Perspektive nichts: Während V. 37 im Präsens die Überwin-

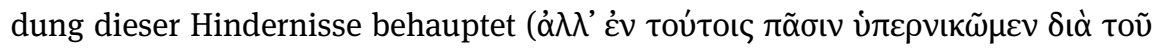

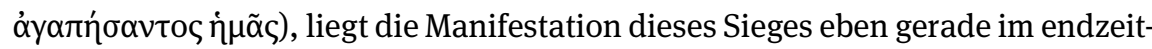

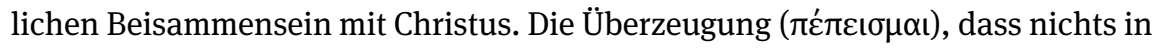
der Lage sein wird, ${ }^{104}$ uns von der Liebe Gottes in Christus Jesus, unserem Herrn,

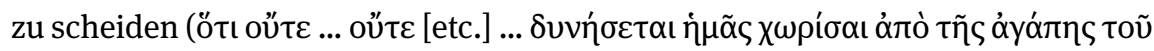

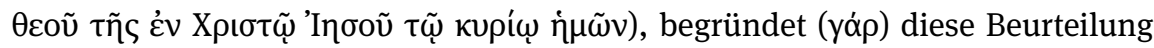
der Lebenswirklichkeit des Christen.

Interessant ist im Vergleich 2. Tim 1,12. Wie schon in Röm 14,14 erscheint

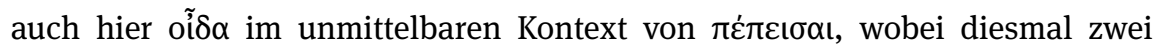

101 Äußerst instruktiv ist Platon, Leg. 804e. Der Einwand, Reiten und Turnen sei nur etwas für

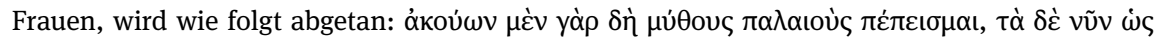

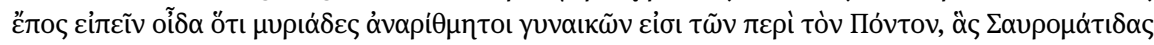
$\kappa \alpha \lambda$ oṽ kampferprobten Sauromatinnen aufgrund des Hörens alter Sagen. Dem steht mittlerweile, eindeutig als Steigerung erkennbar, ein „Wissen“ (wenn auch nur ein „sozusagendes“) gegenüber. Vgl. ,and I know now of my own observation“ (Bury; Platons Quelle ist vermutlich Herodot). So wohl auch Polybios, Hist. 4.23.8.

102 Bezieht man die Präpositionalphrase auf beide Verben, lässt sich keine sinnvolle Lösung finden.

103 So etwa mit Recht Zahn, Brief, 578, der einen Bezug zum irdischen Jesus herstellen möchte. 104 In Röm 8,35 ist ein modales Verständnis von $\chi \omega \rho i \zeta \omega$ erwägenswert (vgl. NSS 926-927: „,wer kann trennen ... [?]“). Demgegenüber ist bemerkenswert, dass der Aspekt des Vermögens in 8,39 durch ein zusätzliches Verb realisiert wird. 
verschiedene Objekte abhängig sind: „Ich kenne ${ }^{105}$ (den,) dem ich mein Vertrauen

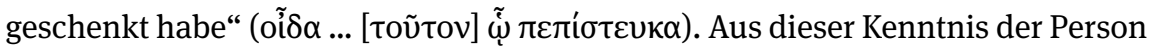

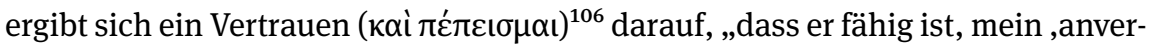

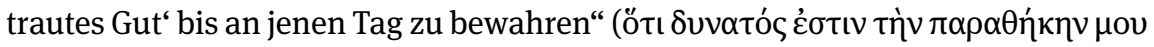

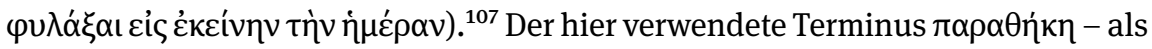
technischer Begriff für ein „Depositum“ - wird in der exegetischen Literatur sehr ausführlich diskutiert. ${ }^{108}$ Das hat vor allem den Hintergrund, dass Timotheus in 1. Tim 6,20 ebenfalls zum bewahren „des anvertrauten Gutes“ und in 2. Tim 1,14

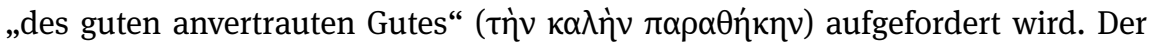
Genitiv in 2. Tim 1,12 wird in der Regel so verstanden, dass hier Paulus nicht das ihm Übergebene, sondern das von ihm Übergebene beschreibt. ${ }^{109}$ Da Timotheus

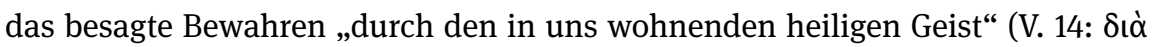

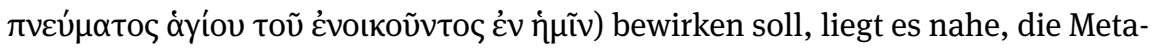
phern zusammen zu denken und inhaltlich durch die in V. 13 beschriebene paulinische Lehre bestimmt zu sehen. Dadurch, dass auch Timotheus wiederum dazu aufgefordert wird, die von Paulus empfangene Lehre weiterzugeben (2. Tim 2,2), besteht eine Kontinuität der Lehre bis zu ,jenem Tag,“ dem Tag der Parusie und des Gerichtes (vgl. 2. Tim 1,18; siehe auch 2. Thess 1,10 zu $\varphi v \lambda \alpha ́ \sigma \sigma \omega)$.

Allerdings wird gerade durch diesen eschatologischen Horizont eine andere Auslegung plausibilisiert: Auch in 2. Tim 4,8 ist die Rede von ,jenem Tag“: An diesem „,wird mir der Herr ... den Siegeskranz der Gerechtigkeit geben ... der

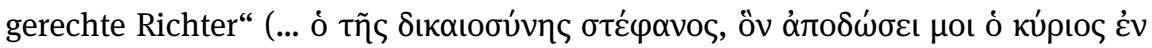

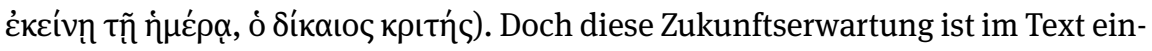
gebunden in die gegenwärtige Situation des Paulus: Er ist jetzt einer, der „den

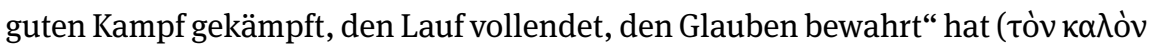

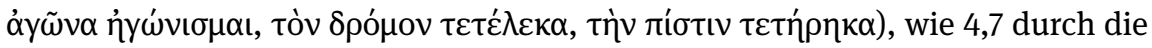
drei resultativen Indikative formuliert. Während für Paulus die Zeit des Abschieds

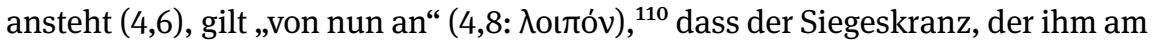

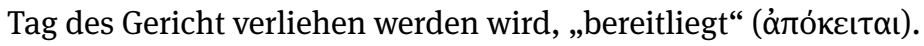

105 Vgl. Menge: „denn ich weiß, wer es ist, auf den ...“

106 Es ist zweifelsohne eine konsekutive Konnexion anzunehmen (vgl. GGNT 334a zum kai consecutivum).

107 Vgl. zum Infinitiv in Röm 8,38-39 auf der einen Seite und 2. Tim 1,12 auf der anderen Seite GGNT 218e mit GGNT 219.

108 Vgl. Wolter, Pastoralbriefe, 115-130 und Weiser, Brief, 125-129.

109 Das Insistieren in der Literatur auf dieser Unterscheidung wirkt mit Blick auf die Sachebene etwas übertrieben, denn es wird wohl vorausgesetzt sein, dass auch Paulus wiederum letztlich nur Empfänger war. Vgl. auch die analoge Rede des „Bewahrens“ ( und als Aufforderung an Timotheus in 1. Tim 6,14. Mit göttlichem Agens in 1. Thess 5,23.

110 BDAG 4612,3a $\alpha$. 
Es ist daher naheliegend, auch in 2. Tim 1,12 einen solchen Hintergrund anzunehmen: Weil Paulus denjenigen kennt, auf den er sein Vertrauen gesetzt hat - weil dieser sich stets als treu erwiesen hat - ist er auch überzeugt, dass er auch künftig bewahrt werden wird - unabhängig von seinem bevorstehenden Tod (vgl. 2. Tim 1,10 zum zunichte gemachten Tod). ${ }^{111}$ Es ist dabei nicht notwendig, Paulus selbst als das „Übergebene“ anzusehen, auch wenn die Parallele zum mit dem Abschied verbundenen Trankopfer in 4,6 eine solche Überlegung durchaus begründen würde. Auf jeden Fall darf der Aspekt des Leidens ( $\pi \alpha ́ \sigma \chi \omega)$ in 1,12 nicht übersehen werden. Denn vor diesem Hintergrund äußert Paulus überhaupt erst, dass er sich „nicht schäme,“ und begründet dies mit der hier diskutierten Phrase. Das irdische Leiden, das bald im Tod enden wird, ist kein Grund zur Scham, da der Tod keine Macht mehr hat $(1,10)$, Gott jedoch die Macht hat, das zu bewahren, was ihm von Paulus „in Gewahrsam“ gegeben wird. ${ }^{112}$ Dabei gehört zum abgeschlossenen irdischen Werk natürlich auch die endzeitliche, aber schon bereitliegende, Entlohnung (vgl. 4,8). Das schließt nicht aus, dass zum Akt des Bewahrens des „Verdienstes“ (im Sinne des Werks und der entsprechenden Entlohnung) auch die Befähigung der den Apostel ablösenden Mitarbeiter zählt ${ }^{113}$ und dass sich daher eine weitere Metapher in 1,14 anschließt. Dennoch kann der Akt des Bewahrens durch Gott in 1,12 nicht einfach mit dem des Timotheus in 1,14 gleichgesetzt werden. Die beiden Fälle unterscheiden sich nicht nur dadurch, dass zwei unterschiedliche „Bewacher“ genannt sind, ${ }^{114}$ sondern auch darin, dass das Bewachte in 1,14 die bereits übergebene Tradition ist, in 1,12 die „Übergabe“ selbst aber wohl noch aussteht und mit dem Abschied (4,6) zusammenfallen wird, wenn Paulus nicht mehr selbst wird wirken können.

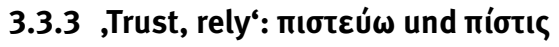

Das Verb $\pi \varepsilon ́ \pi \varepsilon ı \sigma \alpha$ bietet für die Analyse von Protonarrativen mit Zukunftsbezug bei Paulus also durchaus ein gewisses Potenzial. Aus derselben semantischen

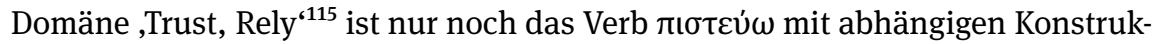
tionen mit Zukunftsreferenz verbunden. ${ }^{116}$

111 So richtig Fee, Timothy, 232.

112 Vgl. BrDAG 1548.

113 Paulinisch kann das mit göttlicher Unterstützung vollbrachte Werk dennoch gefährdet sein. Vgl. oben, Kapitel 11, Abschnitt $6.3 \mathrm{zu} \mathrm{Gal} \mathrm{2,2.}$

114 Richtig Fee, Timothy, 232.

115 LN 31.82-101.

116 Vgl. zur hier gebotenen Diskussion jetzt auch Frey, Schliesser und Ueberschaer, Hg., Glaube. 
Und auch wenn das Verb 54 Mal im corpus Paulinum erscheint und das

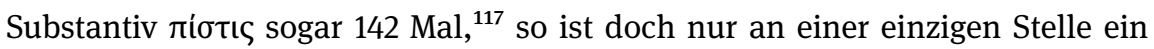
(1) ötı-Satz mit Indikativ Futur zur Markierung der Nachzeitigkeit angefügt. Es handelt sich dabei um die Aussage in Röm 6,8: „Wir glauben, dass auch wir mit

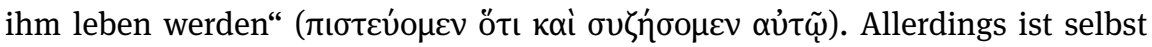
diese Aussage Teil eines indefiniten Kondtionalgefüges und abhängig vom „Mit-

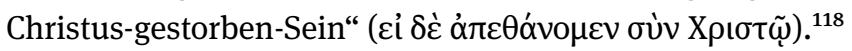

Was die mögliche Anbindung eines nachzeitigen Geschehens durch einen (2)

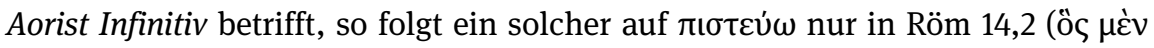

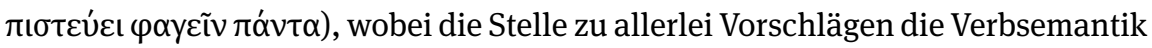
betreffend geführt hat. ${ }^{119}$

Auch was Substantive aus dieser semantischen Domäne angeht, so beziehen sie sich recht selten auf die Zukunft. Ein dem Deutschen („der Glaube, dass ...“)

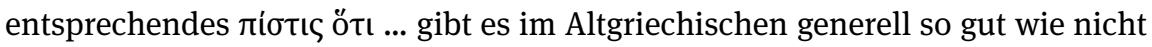

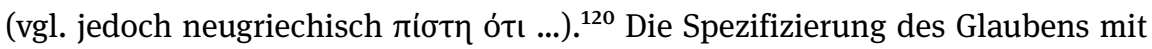

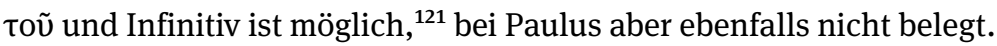

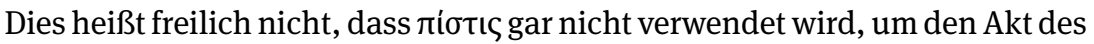
Glaubens an zukünftiges Geschehen zu bezeichnen. An einigen Stellen wird dies auch auf der syntaktischen Ebene deutlich. So betont der „Berge versetzende“

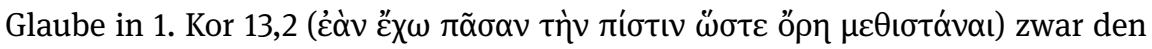

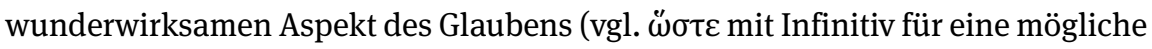
Folge), ${ }^{122}$ beinhaltet sicherlich aber auch den Glauben an das noch ausstehende Geschehen. Das Haben „allen Glaubens“ selbst ist hier freilich Teil eines Konditionalsatzes.

In diesem Kontext ist wohl auch Kol 2,12 zu bedenken: Aus dem Begra-

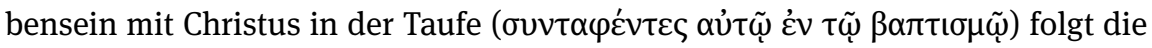

117 Es ist freilich nicht die Gesamtheit der Vorkommnisse dieser semantischen Domäne zuzuordnen, der angesprochene Akt erfüllt also nicht immer die Definition, to believe to the extent of complete trust and reliance' (LN 31.85).

118 Zur Passage siehe die Diskussion der Konditionalsätze im Römerbrief, Abschnitt 5.2.

119 Vgl. etwa Zahn, Römer, 569: „Der eine getraut sich, alles zu essen ...“ BDAG 5939,5 bietet für Röm 14,2, Joh 9,18 und Apg 9,26 die mögliche Bedeutung ,think/consider (possible). Vgl. zur syntaktisch ähnlichen Situation in 1. Tim 3,5 (auch dort wohl mit lexikalisch-semantischer Klärung) oben, Kapitel 13, Abschnitt 3.1.

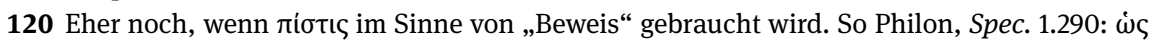

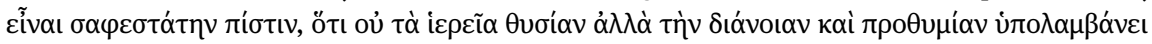

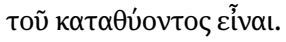

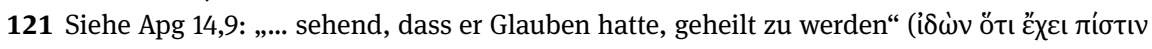

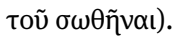

122 Zur Konstruktion vgl. GGNT 279a. 


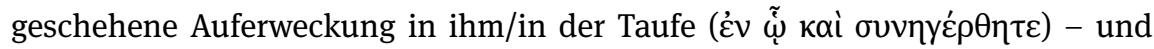
zwar, wie eine lange Präpositionalphrase spezifiziert, „durch den Glauben der

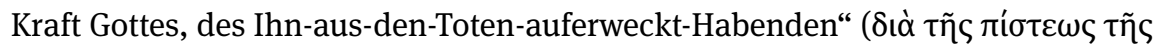

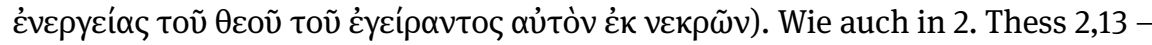

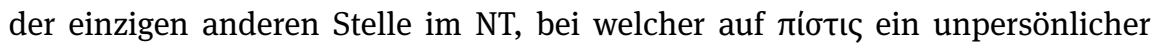
Genitiv folgt (dort von $\alpha \lambda \eta \theta \varepsilon i ́ \alpha$ ) - drückt der Genitiv hier wohl den Gegenstand des Glaubens aus. Der Glaube „an“ die Macht Gottes ist hier durch die Apposition zu $\theta$ cós nicht zuletzt als auf das aus diesem Vermögen hervorgegangenen Handeln in der Vergangenheit gerichtet qualifiziert. Zugleich wird damit aber wohl auch der Glaube an Gottes Vermögen, auch den Getauften neues Leben schenken zu können, gemeint sein. So gesehen läge hier durchaus ein Verweis auf die Zukunft der Täuflinge vor - und die Parallelität mit der Auferweckung Jesu macht zudem wahrscheinlich, dass dabei nicht nur an die Befähigung zu einem neuen Leben gedacht ist (so vermutlich Röm 6,4), sondern auch - die Metapher begründend - an die Auferweckung am Ende der Zeiten (so eventuell Röm 6,8, sicher Röm 6,23). ${ }^{123}$

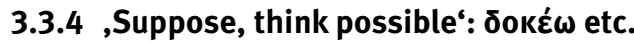

Eine eindeutigere epistemische Abschwächung liegt bei Verben der semantischen Domäne ,Suppose, Think Possible‘ vor, ${ }^{124}$ einer Unterkategorie der grammatisch als Verben des ,Meinens“ $\mathrm{zu}$ bezeichnenden Verben, ${ }^{125}$ die im klassischen Griechisch in der Regel mit Infinitiv konstruiert werden, im NT vermehrt mit abhängigem ötı-Satz. ${ }^{126}$ Das wird etwa deutlich, wenn man sich die Bedeu-

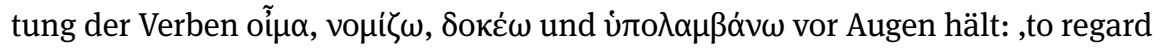
something as presumably true, but without particular certainty. ${ }^{\text {‘27 }}$

123 Gegenüber zu Röm 6 nimmt insbesondere die deutschsprachige Exegese an dieser Stelle eine deutliche Verschärfung des präsentischen Aspektes der Eschatologie wahr (vgl. das indikativische $\sigma u v \eta \varepsilon^{\prime}(\theta \eta \tau \varepsilon$ ). Als weitere Differenz wird zudem teilweise das Fehlen des Motivs des Glaubens in Röm 6 angeführt. So erwägt Schweizer, Brief, 113, dies könne sein, „weil der Glaube der Taufe vorausgeht. “Interessant ist aber, dass der von ihm eingeräumte Verweis auf den Glauben „an die künftige Auferstehung“ in Röm 6,8 der Formulierung in Kol 2,12 durchaus nahezustehen scheint, wenn man der Stelle im Römerbrief einen eschatologischen Horizont zuschreibt: Dem Glauben an Jesu Auferweckung korrespondiert hier implizit sicher auch der Glaube an die eigene zukünftige Auferstehung - der im Kolosserbrief keinesfalls ganz entfällt (siehe Wright, Resurrection, 236-240).

124 LN 31.29-34.

125 GGNT $218 \mathrm{f}$.

126 GGNT 218h.

127 LN 31.29. 
Im Kontrast zu Verben, die stärkere Sicherheit einer inneren Einstellung zu einem Sachverhalt ausdrücken, erlauben diese Lexeme dem Sprecher, Überzeugen zu diskutieren, welche er nicht teilt. Paulus macht auch in der Tat großzügig Gebrauch von dieser sprachlichen Ausdrucksmöglichkeit, jedoch vor allem, um bestehende Meinungen, die auf Gegenwärtiges oder Vergangenes bezogen sind, in ihrer Unsicherheit aufzugreifen.

Dabei kann die rhetorische Funktion je nach Kontext variieren. So verwendet Paulus ein Lexem dieser Kategorie für sich selbst in 1. Kor 7,40, um bewusst

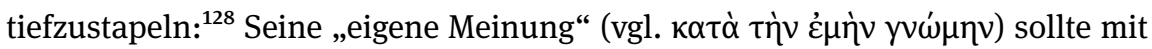
derjenigen der aufgrund des Geist absolut Sprechenden ${ }^{129}$ doch wohl zumindest

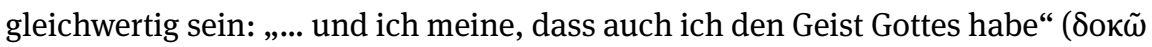

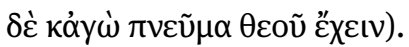

Auch andere auffällige Verwendungsweisen lassen sich bei Paulus identifizieren: So nutzt er insbesondere im 1. Korintherbrief die Verbsemantik, um in indefiniten Konditionalsätzen ${ }^{130}$ die Möglichkeit aufzugreifen, dass eine bestimmte Position vertreten wird - die dann jedoch als ungenügend qualifiziert wird.

So geschieht dies etwa in 1. Kor 3,18: „Wenn jemand unter euch meint, weise

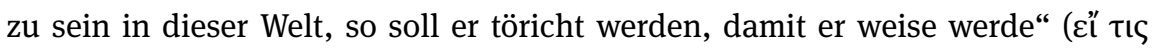

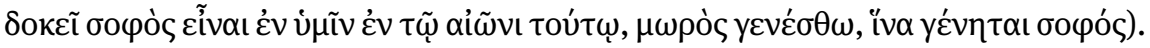
$\mathrm{Zu}$ meinen, man sei weise, ohne das Kreuz mit einzubeziehen (also ohne für die Welt $\mu \omega \rho$ ó zu sein), ${ }^{131}$ ist eine Selbsttäuschung. Wer den sachlichen Zusammenhang bestreitet, welcher dem Konditionalgefüge zugrunde liegt, der täuscht sich selbst. Entsprechend stellt Paulus dem Konditionalsatz die Aufforderung voran:

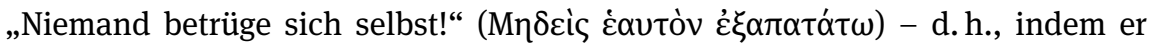
diesen Zusammenhang leugne. Ein Satz wie 1. Kor 3,18 würde mit oĩ $\alpha \alpha$ („Wenn jemand weiß,...") nicht funktionieren.

Besonders deutlich wird dies auch in 1. Kor 8,2: „Wenn jemand meint, etwas

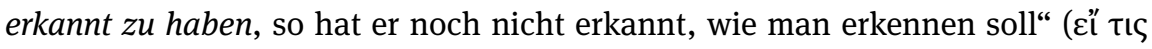

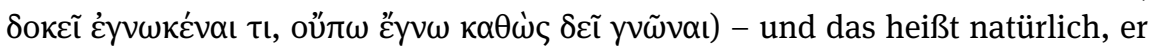
hat noch nicht wirklich erkannt.

Geschickt ist auch 1. Kor 14,37 konstruiert: „Wenn jemand meint, ein Prophet oder Geistbegabter zu sein, so muss er erkennen, dass das, was ich schreibe, ein

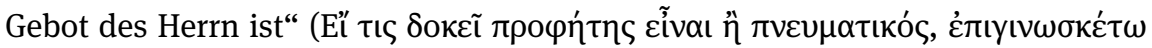

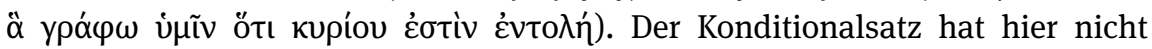
einfach die Funktion, wie etwa ein Relativsatz (őбoı ...), eine bestimmte Gruppe

128 Zur Litosis siehe GGNT 296h.

129 Schrage, Brief I, 206.

$130 \mathrm{Zu}$ diesen siehe unten, Abschnitt 5.2.1.

131 So richtig Schrage, Brief I, 312. 
$\mathrm{zu}$ identifizieren, an welche dann eine Aufforderung ergeht. Vielmehr impliziert der notwendige Zusammenhang von Apodosis mit der Protasis, dass eine Person mit solchem Selbstbild der Aufforderung auch nachkommen muss, da sonst der Umkehrschluss gilt: Sie ist nicht, was sie zu sein meint.

Die Möglichkeit, dass die vertretene Meinung unzutreffend ist, wird in Gal 6,3 explizit durch ein konzessives Partizip angesprochen: „Denn wenn jemand

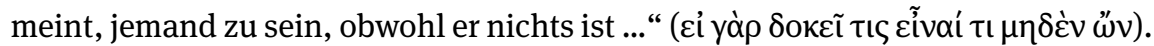
In diesem Fall ist die Meinung notwendigerweise eine Selbsttäuschung (,,... dann

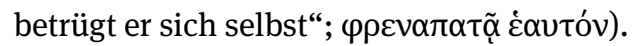

Als akzeptable - wenn auch aus Sicht des Paulus nicht vorzuziehende Meinung wird in 1. Kor 7,36 von Paulus die Überzeugung bewertet, man handle besser an seiner eigenen Jungfräulichkeit, wenn man sie in der Blüte der Jahre „in die Ehe gebe." ${ }^{132}$

Es ist vor dem Hintergrund dieser Textstellen offenkundig, dass jene Ausdrucksformen großes Potenzial bieten, um auch zukünftige Handlungsstränge lediglich tendenziell anzudeuten, ohne sich als Sprecher auf deren Eintreten festzulegen. Umso auffälliger ist, dass Paulus davon keinen Gebrauch macht. Eine gewisse Zukunftsperspektive könnte im corpus Paulinum allenthalben für 1. Tim 6,5 diskutiert werden: Diejenigen, die „meinen, Frömmigkeit sei ein

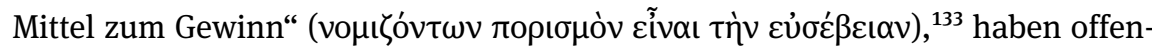

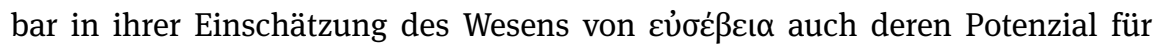
die Zukunft bedacht. Grundsätzlich muss jedoch festgehalten werden, dass die bei den Verben des Meinens überwiegend gegebene relative Zeitbeziehung zwischen Hauptverb und abhängigem Infinitiv ${ }^{134}$ sonst durchgehend nur Verweise auf die Gegenwart ${ }^{135}$ oder Vergangenheit ${ }^{136}$ erkennen lässt. In der Umwelt ist der

132 Dieses Verständnis von 1. Kor 7,36 setzt ein Verständnis des substantivierten Adjektivs $\pi \alpha \dot{\alpha} \theta \varepsilon v 0 \varsigma$ im Sinne von $\pi \alpha \rho \theta \varepsilon v i ́ \alpha$ voraus. Siehe Caragounis, Development, 299-316.

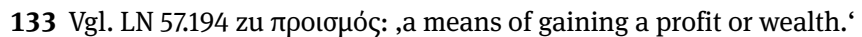

134 Vgl. GGNT 206.

135 Besonders interessant ist 1. Kor 7,26 mit vo $\mu$ i $\zeta \omega$, wo durch die kausale Angabe „wegen der

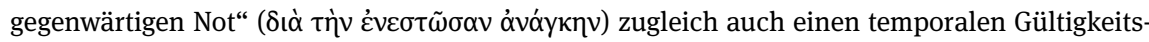
rahmen für die als „gleichzeitig“ markierte Überzeugung liefert. Syntaktisch auffällig ist, dass der folgende Präsens Infinitiv mit Demonstrativpronomen durch einen Nominalsatz mit ötı ex-

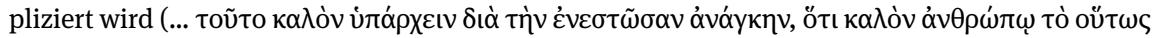
عĩval).

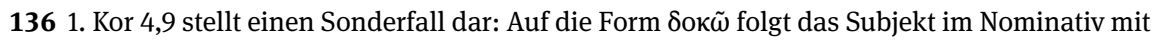
Verb im Indikativ Aorist. Das Verb ist somit als parenthetisch aufzufassen (GGNT 292d). Dies ist aufgrund der Frontstellung im Text äußerst auffällig, da so die Realisierung einer abhängigen Konstruktion aus Schreiber-Perspektive problemlos möglich gewesen wäre - was nicht der Fall ist, wenn ein Schreiber einen bereits begonnen Hauptsatz durch die Einfügung eines parenthe- 
Zukunftsbezug - auch mit Infinitiv Futur - demgegenüber gut belegt. ${ }^{137}$ Gerade wenn man bedenkt, welche Bedeutung in der Paulusforschung der Auseinandersetzung mit alternativen eschatologischen Entwürfen für die Veranlassung von Briefen des Apostels zugeschrieben wird, ist diese Konstellation äußerst bemerkenswert.

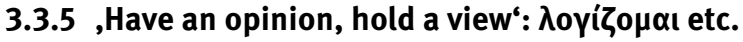

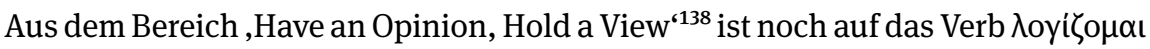
$\mathrm{zu}$ verweisen, welches in Röm 2,3 als Kontrast auf eine Aussage des Wissens ${ }^{139}$ folgt: Der „richtende Mensch“ wird mit der Frage angesprochen: „Denkst Du aber

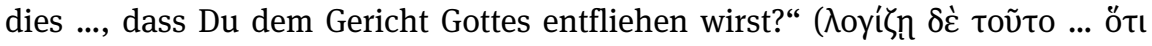

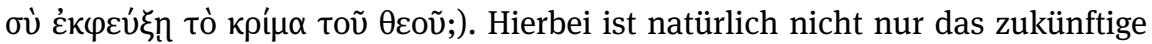
Ereignis des Entfliehens als bloße Meinung markiert, sondern durch die Frage ${ }^{140}$ auch insgesamt nicht als tatsächlich vertreten ausgesagt. Interessant ist auch

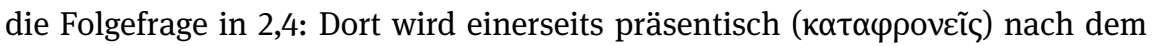
Verachten des Reichtums der Güte und Geduld Gottes gefragt. Andererseits wird durch eine modale/kausale Partizipialkonstruktion auch nach der eventuellen „Unkenntnis“ gefragt, welche letztlich ein zukünftiges Geschehen betrifft: „...

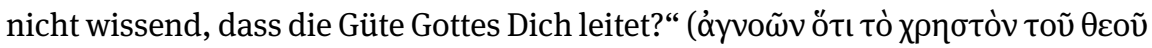

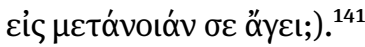

tischen Kommentars abschwächen möchte. Eventuell liegt hier also der umgekehrte Fall vor: Paulus beginnt die Äußerung einer als bloßen Meinung gekennzeichneten Aussage, entscheidet sich dann jedoch dafür, durch einen unabhängigen Behauptungssatz die Einschränkung fallen zu lassen. Eventuell ist auch für die Verben in GGNT 218 mit einem „elliptischer ǒtı-Satz“ zu rechnen (analog zum Deutschen „Es scheint mir, [dass] Du kommst.“). Die Unsicherheit in der Textüberlieferung scheint hierfür zu sprechen. Die Übersetzungen tragen dieser Besonderheit leider keine Rechnung.

137 Recht häufig etwa in der Makkabäer-Literatur (z. B. 2. Makk 7,19 und 3. Makk 5,5). Häufig bei Josephus (z. B. A. J. 2.89). Bei Philon vgl. Legat. 25 und Prob. 132. Eine falsche solche Zukunftserwartung wird bei Philon, Fug. 38 als „Einfältigkeit“ ( $\varepsilon \cup ̉ n ́ \theta \varepsilon ı \alpha)$ bezeichnet.

138 LN 31.1-7.

139 Röm 2,2: „Wir wissen aber, dass das Gericht Gottes entsprechend der Wahrheit ist über die-

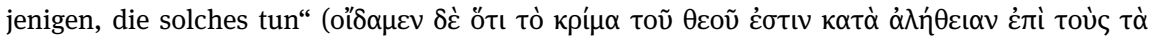

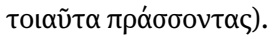

140 Siehe zu Fragesätzen allgemein oben, Abschnitt 2.

141 Das Präsens ist konativ aufzufassen. Siehe GGNT 197a. Vgl. aber auch oben, Kapitel 6, Abschnitt 3.4.1.1. Robertson, Grammar, 880 spricht von ,inchoative or conative present“ und ordnet die Stelle der Kategorie zu, in welcher „an act just beginning“ beschrieben wird. Zur „inchoativen“ Verwendung von Formen des Durativ-Stamms siehe die kritische Diskussion bei GGNT 298e. Siehe auch oben, Kapitel 6, Abschnitt 3.3.3.4. 
Für die Suche nach Ereignisbezügen in der Zukunft, die als Elemente „potenzieller“ Erzählungen gelten können, sind bei Paulus hingegen diejenigen Fälle gut belegt, bei denen zusätzlich zum epistemischen Vorbehalt auch noch eine Wertung kommuniziert wird. Es handelt sich dann entweder um „erhoffte“ oder „befürchtete“ Ereignisse. Diese sollen im Folgenden besprochen werden.

\subsection{Erhoffte Ereignisse}

\subsection{1 ,Hope, look forward to': $\dot{\varepsilon} \lambda \pi i$ í und $\dot{\varepsilon} \lambda \pi i \zeta \zeta \omega$}

Der unsichere Status zukünftiger Ereignisse kann auch dadurch expliziert werden, dass das Geschehen im Text als Hoffnung oder Befürchtung charakterisiert wird, ${ }^{142}$ also durch die Nennung einer Haltung gegenüber noch ausstehenden Ereignissen, welche einerseits damit rechnet, dass das Ereignis mit einer gewissen Wahrscheinlichkeit auch eintreten wird und andererseits eine klar Bewertung dieser Prognose vornimmt.

Während abhängige Befürchtungssätze einen eigenen Satzbauplan aufweisen (siehe die Behandlung im nächsten Abschnitt 3.5), wird das hoffnungsvolle Erwarten eines Ereignisses nach demselben Muster ausgedrückt, wie das „Erwarten“ in neutraler Bedeutung (siehe oben, Abschnitt 3.3), nur dass die entsprechenden abhängigen (ötı-/Infinitiv-)Konstruktionen eben spezifisch von Ausdrücken des Hoffens abhängig sind. Der Unterschied zu den bisher besprochenen abhängigen Behauptungssätzen (und alternativen abhängigen Infinitiv-Konstruktionen) ist also primär ein lexikalisch-semantischer: Durch die Anknüpfung an einen Ausdruck des Hoffens, wird einerseits die positive Einstellung des Sprechers gegenüber dem noch ausstehenden Ereignis ausgedrückt. Zugleich zeichnet die Kennzeichnung von Ereignissen als erhofft aus, dass dadurch in der Regel der Anspruch erhoben wird, sie seien nicht einfach „Wunschdenken,“ sondern es gäbe Gründe, davon auszugehen, dass sie in einer passiven Prognose erreicht würden. ${ }^{143}$ Diese semantischen Besonderheiten rechtfertigen die Diskussion erhoffter Ereignisse unter einer separaten Überschrift.

Das Erhoffen von zukünftigem Geschehen kann sprachlich sehr unterschiedlich ausgedrückt werden. Im Römerbrief begegnet uns eine Hoffnung direkt

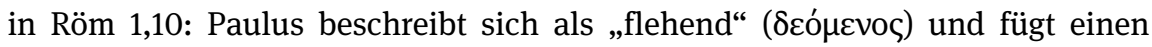
abhängigen Fragesatz an, der seine an Gott gerichtete Bitte in indirekter Rede

142 Siehe oben, Kapitel 11, Abschnitt 4 zu Lämmerts Kategorien von „Wunsch“ und „Angst.“ $143 \mathrm{Vgl}$. LN $25.50 \mathrm{zu} \dot{\varepsilon} \lambda \pi i \zeta \omega$ : to look forward with confidence to that which is good and beneficial. 
formuliert: „... flehend, ob ich vielleicht endlich einmal durch den Willen Gottes

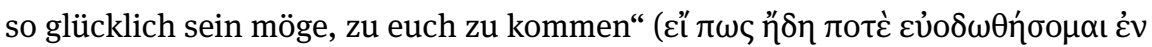

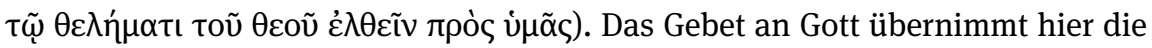
Funktion einer die Verwirklichung des $\varepsilon \dot{i}-N e b e n s a t z e s$ vorantreibenden Handlung, ${ }^{144}$ sodass der Nebensatz selbst eine Hoffnung oder Erwartung ${ }^{145}$ ausdrückt.

Dieselbe Konstruktion liegt auch in Röm 11,14 vor: ${ }^{146}$ Das Preisen seines Amtes als Apostel für die Heiden (Röm 11,13) geschieht „in der Hoffnung, dass ${ }^{147}$ ich vielleicht mein Fleisch reizen“ und (als Folge daraus; d. h. kaí consecutivum)

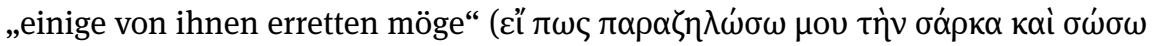

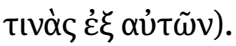

Noch eine weitere Futurform im Römerbrief scheint nicht direkt eine Ankündigung darzustellen, sondern Gegenstand des Hoffens zu sein: In Röm 8,21 kann

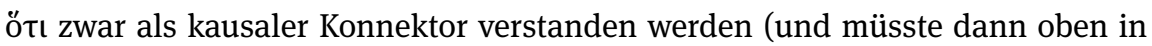
Kapitel 13 eingeordnet werden). Kontextuell plausibler ist jedoch die Anknüpfung an દ̇ $\lambda \pi$ í in V. 20: Die Unterwerfung der Schöpfung unter die Nichtigkeit „auf Hoffnung“ hin, würde dann expliziert werden als ein Hoffen, „dass auch die Schöpfung selbst befreit werden wird von der Knechtschaft der Vergänglichkeit“

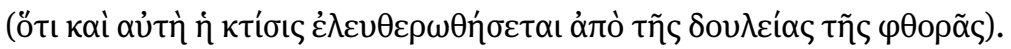

Dieses Verständnis von Röm 8,20-21 wird auch durch eine andere Stellen im corpus Paulinum bestätigt, in welcher das Substantiv غ̇ $\lambda \pi$ í eine auf die Zukunft ausgerichtete Hoffnung einleitet. Exakt demselben Muster folgt nämlich Phil

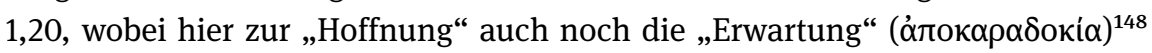
tritt. Beide Substantive werden vom selben Artikel regiert und durch einen ötı-

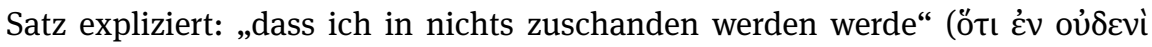

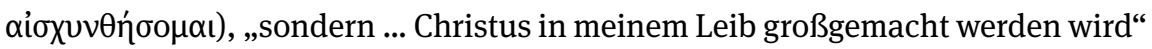

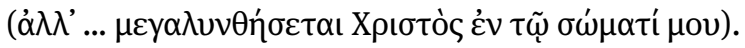

Vor diesem Hintergrund erscheint es zudem sehr erstaunlich, dass bezüglich der Konnexion von 1. Thess 5,8 und 5,9 unter Kommentatoren (und wohl auch

144 GGNT $273 \mathrm{f}$.

145 So BDR 375.

146 Mit Konjunktiv Aorist in Phil 3,11 für die Hoffnung auf das „Gelangen“ zur Auferstehung von den Toten. Caragounis, Development, 191 führt diese Form auf die Verdrängung von ćóv durch cí zurück, welche den umgekehrten Effekt hatte, ,whereby authors who aimed at a more literary

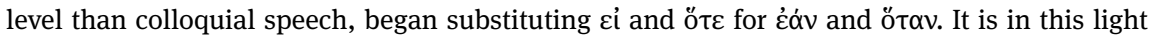
of morphological evolution that the NT use of these conjunctions with the subjunctive is to be understood.“

147 Vgl. NSS 936.

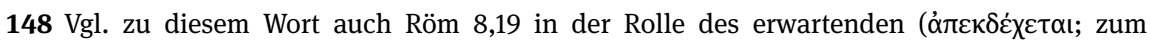

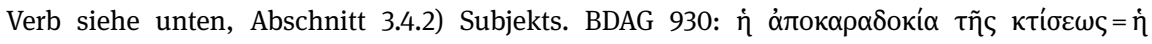

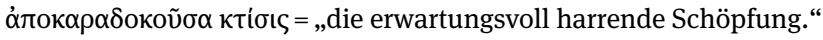


in den frühen Manuskripten, sofern diese graphisch Satzgrenzen markierten) ${ }^{\mathbf{1 4 9}}$ nahezu universelle Einigkeit zu bestehen scheint, dass ǒ $\tau$ in V. 9 eine Begründung einleitet. Man kann natürlich einwenden, dass hier im Gegensatz zu Röm 8,20-21 und Phil 1,20 das Substantiv bereits durch ein anderes Attribut näher

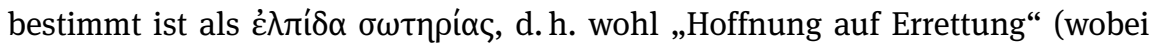
die Hoffnung in Phil 1,20 zumindest auch weiter als „meine“ näher bestimmt ist). Auch fährt V. 9 im Aorist und nicht im Futur fort. Eine Hoffnung muss sich allerdings wohl auch nicht notwendigerweise auf die Zukunft beziehen (vgl. mit resultativem Infinitiv in 2. Kor 5,11: „Ich hoffe aber, auch in euren Gewissen offenbar

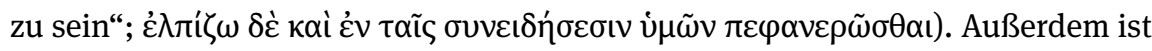

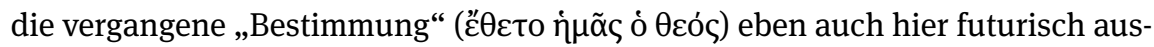
gerichtet: nicht „zum Zorn“ (عiৎ̧ ópyńv), sondern „zum Erlangen des Heils“ ( $\alpha \lambda \lambda$ ”

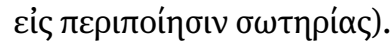

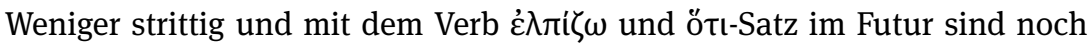
einige andere Verse bei Paulus formuliert. So wird in 2. Kor 1,10 durch einen Relativsatz Gott aus V. 9 aufgegriffen und beschrieben als derjenige, „der uns aus

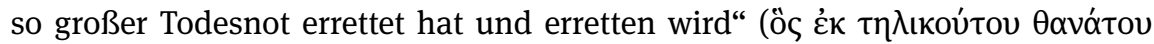

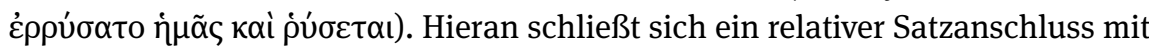

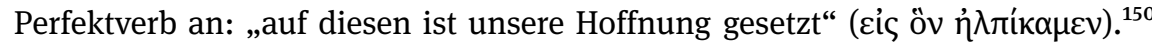
Der Fortgang ist textkritisch umstritten. Entweder fügt sich nun wieder eine mit кaí koordinierte futurische Aussage an, sodass ein neuer Satz beginnen würde:

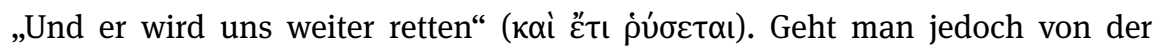
Ursprünglichkeit des ö ı aus (was aus stilistischen Gründen wahrscheinlicher ist), ist dieses weiterhin stattfindende Erretten hier als Hoffnung qualifiziert. Schon aufgrund unserer Unkenntnis der genauen Problematik in Asia ${ }^{151}$ ist es schwierig, festzustellen, was der Unterschied zwischen dem uneingeschränkt postulierten und dem erhofften Erretten ausmacht. ${ }^{152}$ Wenn man dies für mit der Semantik von ع̈tı vereinbar hält, könnte in dieser zweiten Aussage durchaus ein eschatologischer Bezug erkannt werden. ${ }^{153}$

Nur wenige Verse später, in 2. Kor 1,13 wird abermals durch $\dot{\varepsilon} \lambda \pi i \zeta \omega+$ ö $\tau$ + Indikativ des Futurs eine Hoffnung geäußert (diesmal mit Präsens im Matrixsatz),

149 Dass der Codex Boernerianus eine ganz analoge Trennung auch innerhalb von Phil 1,20 vornimmt, ist wohl schlicht auf die Stichoi der Vorlage zurückzuführen (ich danke Seth Ehorn für diesen Hinweis).

150 Vgl. dieselbe Form in 1. Tim 4,10, wo jedoch kein Objektsatz abhängig ist.

151 Vgl. zum generellen Gedankengang Heilig, Paul's Triumph, 158.

152 Aus dieser Schwierigkeit dürfte sich auch die Anpassung ans Präsens oder völlige Auslas-

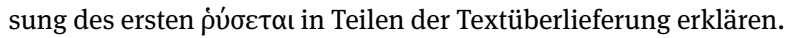

153 So von Schmeller, Brief I, 73 erwogen. 
nämlich die das „,öllige/bis ans Ende der Zeit reichende“ Erkennen der Korinther

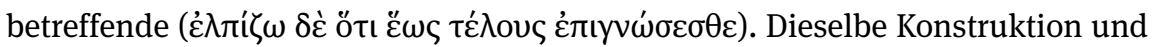
ein ähnlicher Gedanke liegt auch in 2. Kor 13,6 vor, wobei hier der Gegenstand des erhofften Erkennens ( $\gamma \nu \omega ́ \sigma \varepsilon \sigma \theta \varepsilon)$ spezifiziert wird: „dass wir nicht untüchtig

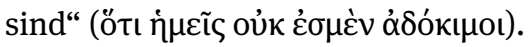

Auch Phlm 22 folgt diesem Muster: Nachdem noch in V. 21 vom „Wissen“ über zukünftiges Handeln die Rede war - dass nämlich Philemon mehr tun „wird,“

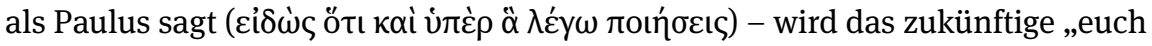

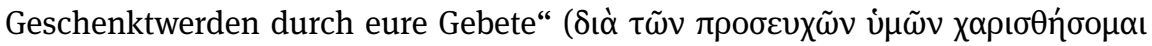

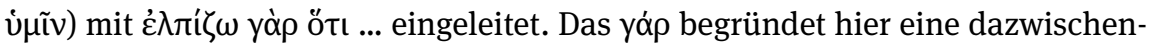
geschaltete (nicht weiter eingeschränkte) Aufforderung, ${ }^{154}$ Paulus eine Herberge

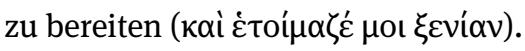

Anstelle des Indikativs des Futurs tritt bei Paulus an einigen Stellen auch die Konstruktion mit dem Infinitiv nach $\dot{\varepsilon} \lambda \pi \hat{i} \zeta \omega$, wobei die futurische Referenz dennoch völlig zweifelsfrei ist. So etwa im Fall des schon besprochenen (siehe Kapitel 11, Abschnitt 8.5) Falls von Röm 15,24, wo das „Sehen“ und „Geleitetwerden“ von غ̇ $\lambda \pi i \zeta \omega$ abhängig ist.

Auch der zweite Beleg innerhalb des paulinischen Briefkorpus entspringt einem Reiseplan im Briefschluss und ist uns bereits bekannt (siehe dazu oben, Abschnitt 3.2.2), denn in 1. Kor 16,7 ist das ,eine gewisse Zeit bei euch Verwei-

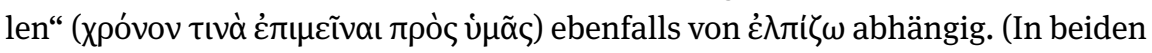
Fällen ist interessanterweise ein Konditionalsatz mit ćáv angefügt, auch wenn die Funktion nicht genau deckungsgleich ist). ${ }^{155}$

Auch das erhoffte „Senden“ des Timotheus, welches in Phil 2 zwei Mal

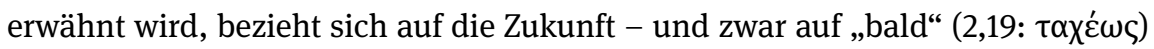
beziehungsweise etwas genauer „wenn ich meine Lage übersehe“ (2,23: $\dot{\omega} \varsigma$ âv

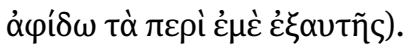

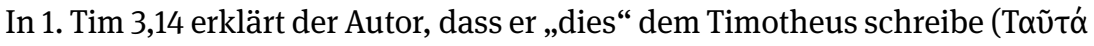
бо үра́ $\varphi \omega . .$. ; gemeint sind die Anweisungen in 1. Tim 2,1-3,13), wobei er hoffe

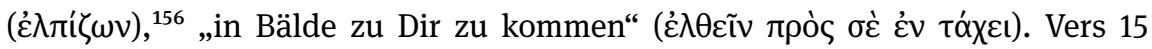
rechnet dann aber explizit mit der Möglichkeit, dass sich diese Hoffnung auf ein

154 D. h., die Begründung hat Äußerungsbezug: „Ich fordere Dich dazu auf, weil ich vorhabe, zu kommen.“ Zum Imperativ siehe oben, Kapitel 5, Abschnitt 3.3.

155 Siehe dazu Kapitel 5, Abschnitt 3.4 und Kapitel 7, Abschnitt 4.2.4.

156 Neben einer kausalen oder modalen Sinnrichtung wäre natürlich auch ein konzessives Verständnis möglich. Das $\delta \varepsilon ́$ im Konditionalsatz in 3,15 scheint den ins Auge gefassten Fall jedoch gerade als von der vorübergehenden - im Partizip ausgedrückten - Annahme von V. 14 abweichend zu charakterisieren. Bei einem konzessiv gemeinten Partizip wäre in V. 15 vielmehr ein yó $\rho$ zu erwarten. 
baldiges Wiedersehen zerschlagen könnte: „Falls ich mich aber verspäte“ ( $\dot{\varepsilon} \alpha \mathbf{~} \delta \dot{\varepsilon}$ $\beta \rho \alpha \delta u ́ v(\omega)$, so hätte das Schreiben den Zweck, Timotheus über die richtige Leitung des Hauses Gottes zu informieren. ${ }^{157}$

Neben $\dot{\varepsilon} \lambda \pi i \zeta \omega+$ ötı oder Infinitiv und $\dot{\varepsilon} \lambda \pi i \zeta$ + ötı gibt es noch einige alternative Möglichkeiten, Hoffnungen einzuleiten, die hier nur kurz angerissen werden sollen. So zeigt die Konstruktion in 2. Kor 10,15 eine weitere Option auf: Hier ist das Substantiv $\dot{\varepsilon} \lambda \pi i \zeta \zeta$ mit Infinitiv kombiniert. Das „Haben“ der Hoffnung ( $\dot{\varepsilon} \lambda \pi^{\prime} \delta \alpha$...

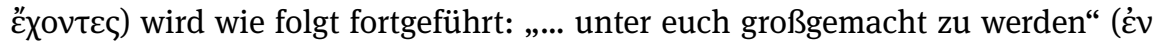

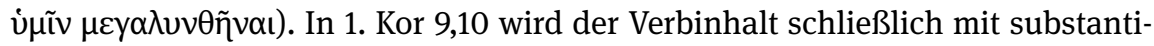
viertem Infinitiv als Genitivattribut als Hoffnung qualifiziert: Der Dreschende soll

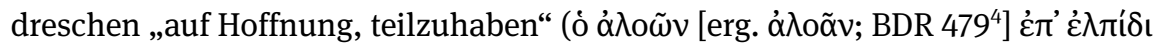

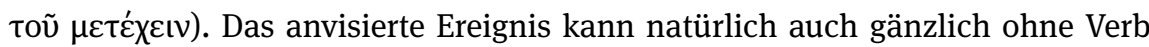
zum Ausdruck kommen, wenn ein entsprechendes Substantiv (in der Regel ein Aktionsnomen) diese Rolle des Genitivattributs übernimmt: So etwa in Röm 5,2

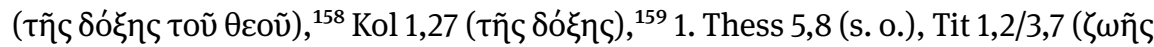

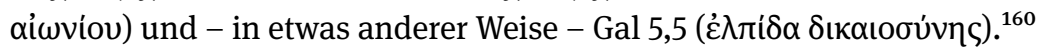

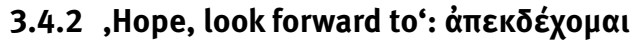

Anders als im Fall von $\dot{\varepsilon} \lambda \pi i \zeta \omega$ ist es bei $\alpha \dot{\pi} \varepsilon \kappa \delta \dot{\varepsilon} \chi 0 \mu \alpha$ (dem zweiten wichtigen Verb aus der semantischen Domäne des Hoffens und Erwartens) ${ }^{161}$ üblich, das erwartete Ereignis durch ein Substantiv anzufügen. So folgt auf das Partizip in

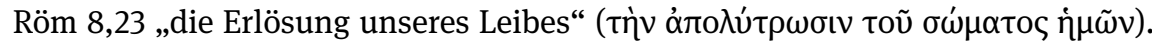
Ebenso wird in 1. Kor 1,7 „die Offenbarung unseres Herrn Jesus Christus“ ( $\tau \grave{\eta} v$

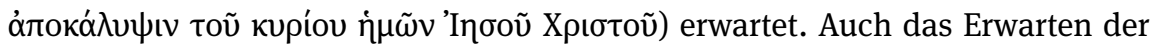
„Hoffung der Gerechtigkeit“ in Gal 5,5 (siehe oben) fällt in diese Kategorie. In

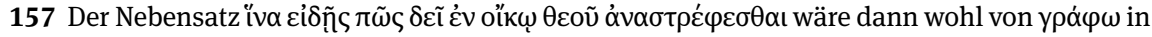
V. 14 abhängig. Alternativ wäre auch die Möglichkeit zu erwägen, dass hier ein imperativischer Gebrauch vorliegt (siehe oben, Abschnitt 4.2.1). Dann wäre der Konditionalsatz zu V. 14 zu ziehen und das Partizip konzessiv aufzufassen (vgl. letzte Fußnote): „Ich schreibe Dir dies - obwohl ich bald zu Dir zu kommen hoffe - für den Fall, dass ich mich verzögere.“

158 D. h. die von Gott gegebene/zu gebende Herrlichkeit. Durch den zusätzlichen genitivus auctoris muss der von غ̇ $\lambda \pi$ í̧ abhängige objektive Genitiv keine Handlung bezeichnen.

159 Die Einordnung hier ist vom Verständnis der Referenz des Substantivs abhängig. Vgl. die letzte Fußnote zu Röm 5,2, wo ein zusätzlicher Genitiv das Gemeinte näher spezifiziert.

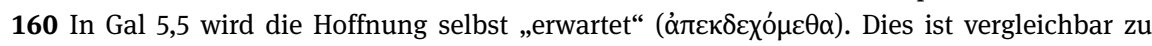

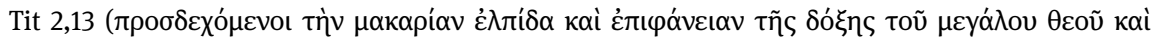

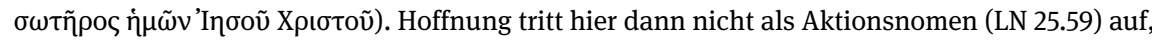
sondern bezeichnet den Inhalt der Hoffnung (LN 25.61).

161 LN 25.59-64. 
Phil 3,20 ist es eine Person ${ }^{162}$ - und damit implizit ihr Kommen - welche erwartet

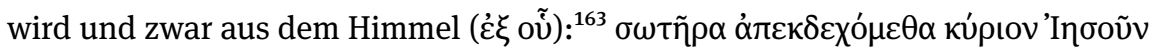

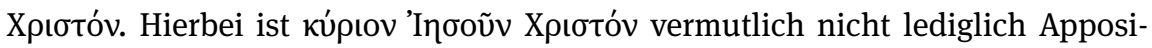
tion zu $\sigma \omega \tau \tilde{\eta} \rho \alpha$ (,,... den Retter, den Herrn Jesus Christus“). Vielmehr wird wohl $\sigma \omega \tau \tilde{\eta} \rho \alpha$ die Funktion einer Objektartergänzung haben: Erwartet wird der Herr Jesus Christus (AkkO) „als Retter“ (wohl im Kontrast zum „Verderben“ in V. 19 hervorgehoben).

\subsection{3 ,Trust, rely': пв́по $\theta \alpha$}

Es wurden bereits die medial-passivischen Perfekt-Formen von $\pi \varepsilon i \theta \omega$ besprochen, welche eine Überzeugung einleiten können (siehe oben, Abschnitt 3.3.2 zu

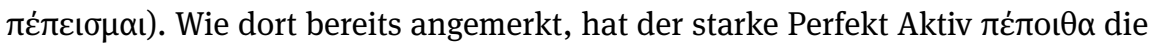
Funktion, ein Vertrauen auszudrücken. Dieses kann sich auch auf das Eintreten zukünftiger Ereignisse beziehen. ${ }^{164}$

So bringt Paulus in Gal 5,10a ein Vertrauen zum Ausdruck, das auf die Galater

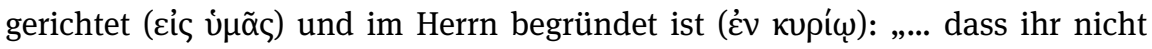

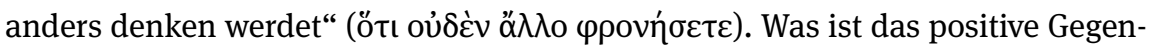
stück? Es kann hier nicht, wie an anderen Stellen, ${ }^{165}$ der Schwerpunkt auf dem

162 Ebenso Hebr 9,28. Vgl. auch oben, Abschnitt 3.2.1 zum die Bewertung des „Erwarteten“ be-

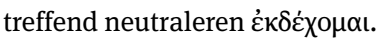

163 Das Relativpronomen ist dem Sinn nach konstruiert. Vgl. GGNT 265.

164 Vgl. das Zitat von Machon bei Athenaios, Deipn. 581c: Es wird geschildert, wie die Prostituierte $\Gamma v \alpha \theta \alpha i$ in $\varsigma$ mit einem wohlhabenden Interessenten um den Preis feilscht. Letztlich gibt sie

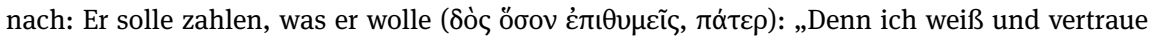

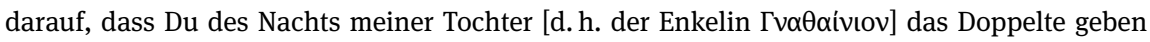

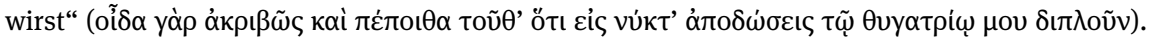
Hier legt sich natürlich ein Vergleich zur ganz ähnlichen Formulierung in Phil 1,25 nahe: kaì

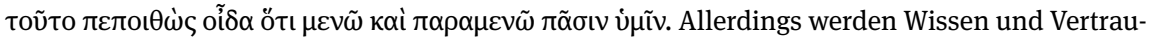
en in beiden Fällen sehr unterschiedlich aufeinander bezogen. Im obigen Zitat von Machon ist

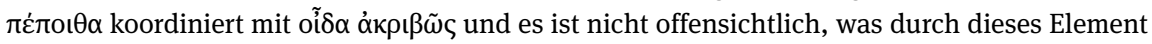

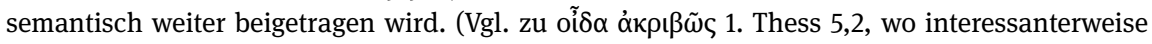

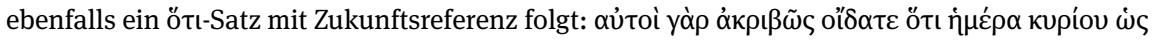

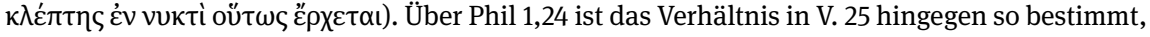
dass das Wissen des Paulus über sein zukünftiges Schicksal aus dem Vertrauen darauf erwächst,

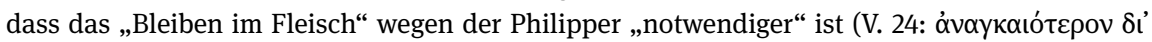
vं $\mu \tilde{\alpha} \varsigma$ ). (Im einen Fall verweist das Demonstrativpronomen also voraus auf das zukünftige Geschehen, im anderen Fall zurück im Text.) Auch diese Formulierung ist ungewöhnlich, würde man doch eher erwarten: „und weil ich davon überzeugt bin/weil ich das weiß, vertraue ich darauf/hoffe ich ....

165 Z. B. Phil 2,2. Vgl. auch 1. Petr 4,1. 
Haben derselben Gesinnung innerhalb der Gemeinde(n) liegen. ${ }^{166}$ Vergleichbar

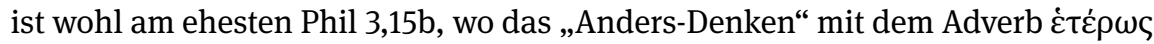
(nur hier bei Paulus verwendet) ausgedrückt wird und sich auf eine Einschät-

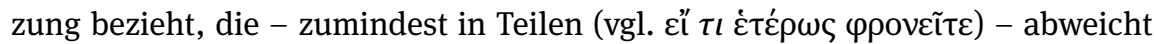
von dem, was der Apostel geschrieben hat. Der Bezugspunkt ist hierbei vermutlich das in 3,13-14 gegebene Beispiel der richtigen Einstellung, ${ }^{167} \mathrm{zu}$ der in 3,15a explizit aufgefordert wird („So viele nun vollkommen sind, lasst uns so

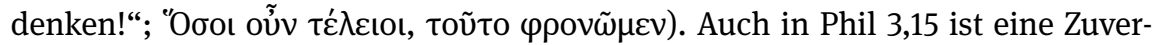
sicht geäußert, dass letztlich Übereinstimmung zwischen dem Apostel und den Adressaten bestehen wird. Denn der im indefiniten Konditionalsatz angedachte Fall einer Meinungsunterschiedenheit, wird in der Apodosis durch die selbstbewusste Behauptung ergänzt: „... dann wird euch Gott auch dieses [d.h. das $\tau$,

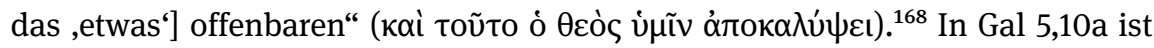
also wohl ähnlich zurückverwiesen auf die in 5,8-9 zum Ausdruck kommende Meinung über die „Unruhestifter“ in Galatien. Dies wird auch dadurch bestätigt, dass in 5,10b zugleich eine Gerichts-Ankündigung in deren Richtung erfolgt, also eine Zweiteilung zwischen der erhofften Einheit des Paulus mit den Galatern und der gemeinsamen Front der Eindringlinge aufgemacht wird. Dass mit der „nicht

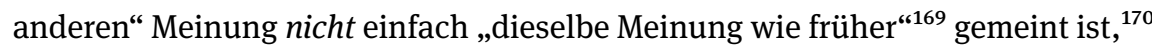
wird zudem dadurch klar, dass Paulus V. 10a durch ein Wortspiel mit $\pi \varepsilon i \theta \omega$ an

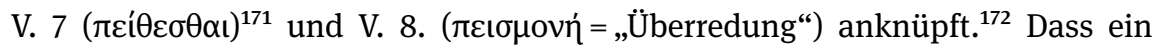
solches Wortspiel beabsichtigt ist, zeigt sich am auffälligen Gebrauch des Per-

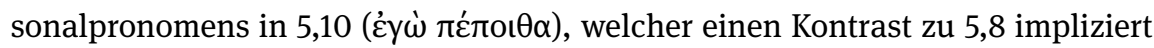

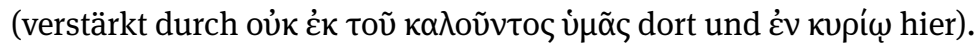

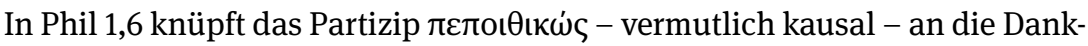

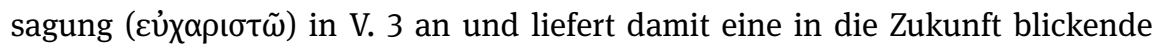
Begründung für den Dank, die neben die rückwärtsgewandte Begründung in V. 5 tritt (,... wegen eurer Teilnahme am Evangelium vom ersten Tag bis jetzt“; غ̇ $\pi \grave{~}$

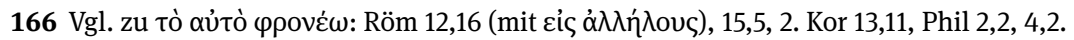

167 Richtig bereits Franke, Briefe, 197.

168 Vgl. auch 2. Kor 1,13.

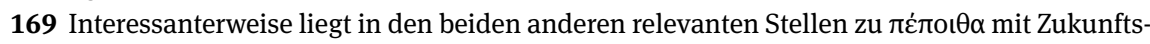
referenz bei Paulus in der Tat eine Korrelation zu vergangenem (Phil 1,6) und die Gegenwart einschließendem (2. Thess 3,4) Geschehen vor. Siehe die Diskussion zum Ende dieses Abschnitts. 170 So Lightfoot, Epistle, 287.

171 Die Bedeutung des Passivs (außerhalb des Perfekts) ist ,to be won over as the result of persuasion. Mit Dativ ist dann das „Gehorchen“ bzw. „Folgen“ gemeint. Vgl. BDAG 5754.

172 Vgl. den Versuch einer Paraphrase bei Moo, Galatians, 334-335: „Who has cut in on you with the purpose of persuading you not to follow the truth? Such persuasion is not from God ... I myself, however, am persuaded about you that ..." 


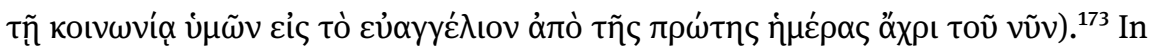

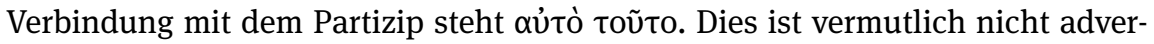
bial zu verstehen im Sinn von „eben darum, “174 sondern ist als Objekt zum Verb zu ziehen: „... weil ich eben darauf vertraue. “175 Es verweist katadeiktisch ${ }^{176}$ auf den folgenden ö $\tau$-Satz: ${ }^{177}$ Paulus vertraut eben darauf, dass „der, der ein gutes Werk in euch begonnen hat, es vollenden wird bis zum Tag Christi Jesu“ (ö $\tau$ ı ò

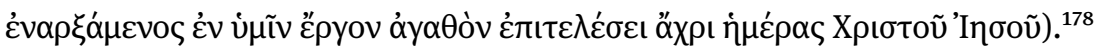

Wie schon in Gal 5,10 ist das in Phil 2,24 geäußerte Vertrauen ,im Herrn“ ('́v кupí( ) verankert. Das anvisierte Geschehen besteht darin, „dass ich bald selbst

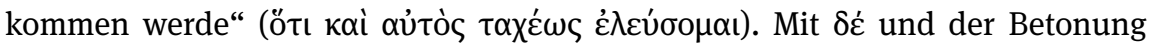
des eigenen Kommens wird dabei an 2,23 angeknüpft, wo von der Sendung des Timotheus die Rede ist. Dessen Sendung wird dort interessanterweise explizit als

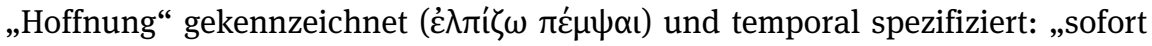
sobald ich meine Lage übersehe“ (灾 ầ ả natürlich auch die Möglichkeit erwogen, dass die Entwicklung der Umstände die Hoffnung auch zunichte machen könnte. ${ }^{180}$ Umso mehr fällt die Zuversicht in Phil 2,24 auf. Die starke Ausdrucksweise steht in Kontinuität zu Phil 1,21-26, mit der klimatischen Formulierung „... darauf vertrauend weiß ich, dass ich bleiben und

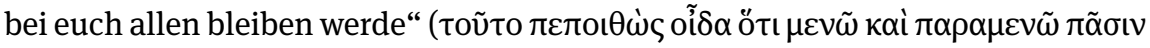
ن่ $\tilde{i} v) .{ }^{181}$ Auffällig ist schließlich, dass „im Herrn“ Erwartungen formuliert werden können, die im Hinblick auf die Gewissheit durchaus divergieren können: દ’v

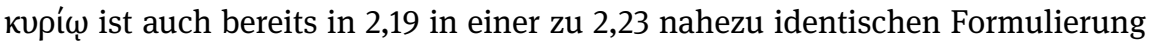

173 Franke, Briefe, 33 sieht eine Anknüpfung an die „Gebetsfreudigkeit“ in V. 4. Doch die in V. 5 gebotene Begründung ist doch wohl eher auf die Hauptaussage in V. 3 zu beziehen.

174 Vgl. BDR 290,56.

175 Vgl. BDR $154^{3}$.

176 Es ist nicht zutreffend, dass „der im Folgenden ausgesprochene Inhalt des Vertrauens keineswegs das eben in Rede stehende ist, “ wie Franke, Briefe, 33 mit Verweis auf die Verwendungs-

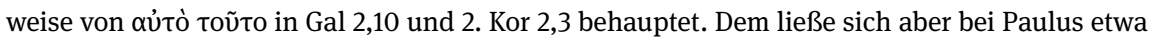
Röm 9,17 (wenn auch im Zitat) und 2. Kor 7,11 entgegenhalten. Auch die Konstruktion mit हiৎ (rückverweisend gebraucht in Röm 13,6 und 2. Kor 5,5) wird in Eph 6,22 so gebraucht (mit explizierendem ivo-Satz). Ebenso auch Kol 4,8.

177 So auch GGNT 141d. Vgl. dort allgemein zum Demonstrativpronomen vor Objektsätze. Für

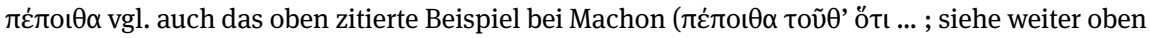
in diesem Abschnitt).

178 Vgl. zum Tempus GGNT 202a. Das Bezeichnete ist als andauerndes Geschehen gemeint, auch wenn das Griechische dies (zu dieser Zeit noch) nicht aspektuell ausdrücken kann.

179 Vgl. GGNT 276f zum vorzeitigen, auf die Zukunft bezogenen Temporalsatz.

180 Vgl. Franke, Briefe, 115.

181 Vgl. dazu oben, Kapitel 13, Abschnitt 3.1. 


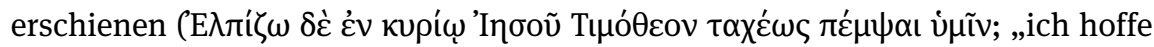
aber im Herrn Jesus, Timotheus bald zu euch zu senden“).

In Phil 1,6 wird die Zukunftserwartung durch die partizipiale Umschreibung des als Handelnden erwarteten Subjekts in der Vergangenheit verwurzelt:

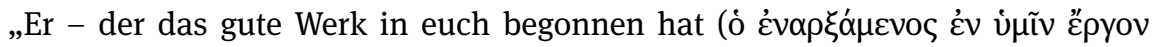
'́y $\alpha \theta$ óv) - wird es vollenden. “ In 2. Thess 3,4 liegt eine ähnliche Koordination von erwartetem Geschehen und bereits Vollbrachtem vor. „Unser Vertrauen“

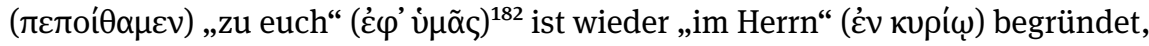

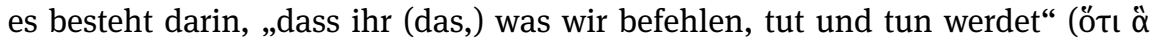

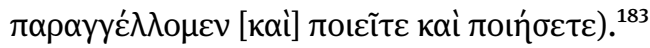

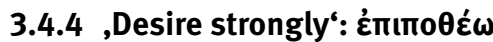

Verwandt mit dem Gedanken des Erhoffens sind auch Verben, die ein „Sehnen“ ausdrücken, ${ }^{184}$ also wie im Fall des Hoffens ein einerseits noch ausstehendes Geschehen andererseits als positiv bewerten. Dabei steht in der Regel diese Bewertung im Mittelpunkt und nicht so sehr die Prognose über das tatsächliche Eintreffen.

Die starke emotionale Involviertheit wird in 2. Kor 5,2 im Kontext noch zusätzlich lexikalisch ausgestaltet: Hier richtet sich die Sehnsucht auf die noch ausstehende „Überkleidung“ mit der Behausung aus dem Himmel und wird wohl als Ursache (oder Begleitumstand) für das gegenwärtige Seufzen angeführt

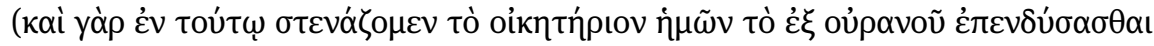

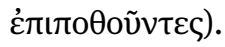

Das Verhältnis von „bloßem“ Hoffen und intensivem Sehnen kann in Röm 1,11 auch in der direkten Gegenüberstellung erkannt werden: Hier motiviert die

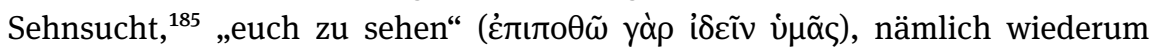
die - zum Gebet führende - Hoffnung, Gott möge es fügen, dass Paulus nach Rom kommen werde (Röm 1,10; vgl. obe, Abschnitt 3.4.1).

Wie hier steht das ersehnte „Sehen“ auch in 1. Thess 3,6 („,... wobei ihr euch

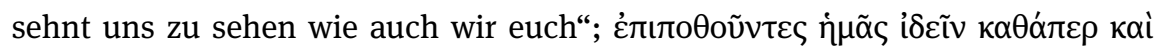

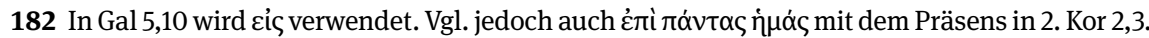
183 Siehe zu $\pi \alpha \rho \alpha y=\dot{\varepsilon} \lambda \lambda \omega$ an dieser Stelle und im 2. Thessalonicher insgesamt unten, Abschnitt 4.4.3.6. Siehe dort auch für den mit ćv kupí $\omega$ hergestellten Zusammenhang mit 2. Thess 3,3. 184 Vgl. LN 25.12-32.

185 Man könnte auch sagen „der Wunsch.“ Diese Terminologie soll jedoch hier vermieden werden, um „Wünsche“ im Sinne von Sprechakten klar abgrenzen zu können. Siehe dazu unten, Abschnitt 4.1. Ihnen entspricht auf der Ebene der Satzart meistens (aber nicht notwendigerweise) ein Desiderativsatz. Siehe dazu unten, Abschnitt 4.3. 


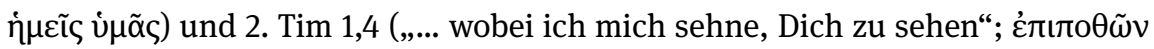

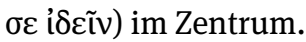

Das damit gemeinte Aufeinandertreffen ist auch das Ersehnte, wenn als Akku-

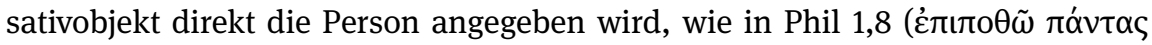

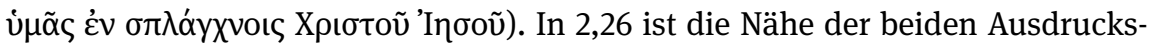
weisen auch durch die Variante mit $ı \varepsilon \_v$ belegt. Hier wird das durch periphrastischen Ipf. ausgedrückte Sehnen des Epaphroditus, „nach euch allen“ (Var.: „euch alle zu sehen“) auch explizit im Kontext mit dem tatsächlichen Senden verbunden (V. 25.28). Zum Zeitpunkt des Schreibens des Briefes ist das Ersehnte noch zukünftig (und auch das Sehnen entsprechend noch anhaltend), bei der Lektüre dann bereits in Erfüllung gegangen.

Während Phil 2,26 ein in der Vergangenheit eingesetzt habendes Sehnen aufgreift, ist in 2. Kor 9,14 eines im Blick, welches noch gar nicht angefangen hat. Vielmehr legt Paulus bereits in den Versen 11-13 dar, wie die Gabe der Korinther für die Gemeinde in Jerusalem dort als positive Folge an Gott gerichtete Danksagungen nach sich ziehen wird. Die zukünftige Verherrlichung Gottes erscheint

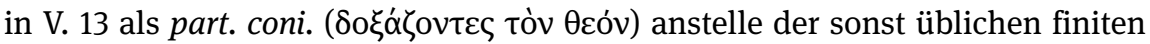

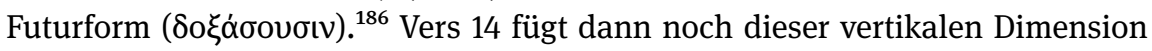
die Beziehungsebene zu den Gebern hinzu: ,und in ihrem Gebet für euch werden

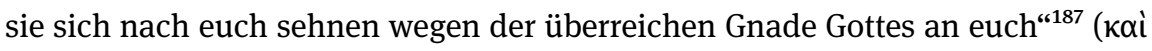

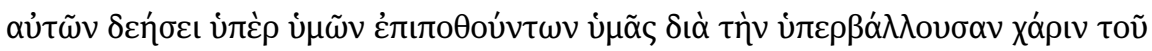

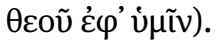

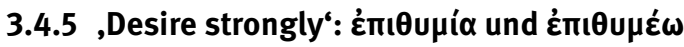

Auch wenn $\dot{\varepsilon} \pi \imath v \mu i \dot{\alpha}$ und $\dot{\varepsilon} \pi \imath v \mu \varepsilon \dot{\varepsilon} \omega$ überwiegend in negativer Bedeutung auftreten, ${ }^{188}$ kann damit auch neutral eine tiefe Sehnsucht zum Ausdruck gebracht werden. ${ }^{189}$ Zumindest das Substantiv begegnet in dieser Bedeutung an zwei Stellen bei Paulus und drückt dabei ein im Kontext klar positives Verlangen aus.

So sagt Paulus in Phil 1,23, er „habe Sehnsucht“ und zwar danach (Eis

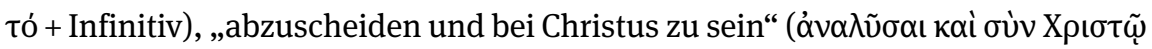
हiv $\alpha \mathrm{l})$. Und in 1. Thess 2,17 ist aoristisch von einem „Bemühen, euer Gesicht zu

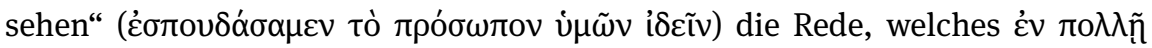

186 Vgl. GGNT 231j und NSS 1021.

187 Für die verschiedenen Interpretationsmöglichkeiten siehe Schmeller, Brief II, 103.

$188 \mathrm{Vgl}$. LN 25.20 ,to strongly desire to have what belongs to someone else and/or to engage in an activity which is morally wrong.

189 LN 25.12: ,to greatly desire to do or have something." 


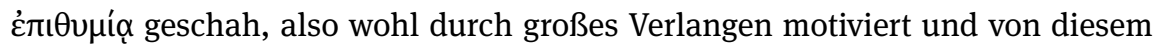
begleitet war. ${ }^{190}$

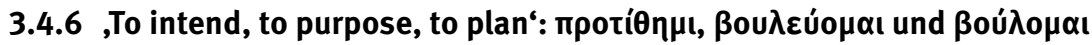

Die in diesem Kapitel schon mehrfach in Erscheinung getretene Rede von den Reiseplänen des Paulus macht noch auf einen weiteren Aspekt aufmerksam, der mit der Semantik des Hoffens verbunden ist. Das „Schmieden“ eines Planes ist einerseits natürlich eine mentale Handlung, welche aufgrund der Diskrepanz zwischen passiver und aktiver Zukunftsprognose nötig wird, wenn ein Individuum also realisiert, dass „his desire will [not] be fulfilled by the actions of another individual“ und es daher in der aktiven Prognose spezifiziert „which steps he should take to realize his goal." ${ }^{191}$ Ist dieser Weg aber erst mal eingeschlagen, so ist das dann gegenwärtige Handeln auf diese zukunftsgerichtete Intention ausgerichtet. Wenn Paulus daher sagt, dass er zum Zeitpunkt des Schreibens etwas „vorhat,“ so ist hier wieder auf eine erhoffte - eventuell bereits durch Taten einer Realisierung näher gebrachte - Zukunft ins Auge gefasst. Daher sind in der Rekonstruktion von Protonarrativen auch die Lexeme aus der semantischen Domäne des Intendierens, Vorhabens, Planens zu berücksichtigen. ${ }^{192}$ Dies gilt natürlich unabhängig vom Tempus in dem sie erscheinen, da sie in der Regel immer mit „embedded narratives“ verbunden sind. ${ }^{193}$ Für Vorausdeutungen auf die Zukunft des Erzählers sind jedoch diejenigen Fälle von Interesse, in denen ein Geschehen als zukünftiger Teil eines gegenwärtigen Plans kenntlich gemacht wird.

Dies kann durchaus auch bei Augmenttempora der Fall sein. ${ }^{194}$ In Röm 1,13 etwa „erzählt“ Paulus den Römern indirekt über seine eigenen mentalen Protonarrative, indem er dem Wunsch Ausdruck verleiht, dass ihnen eine bestimmte Sache nicht „unbekannt“ sein möge: ${ }^{195}$ „... dass ich mir oft vorgenommen ${ }^{196}$ habe,

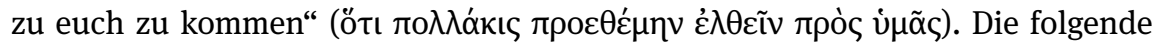

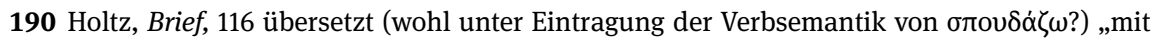
starkem Einsatz" und verschiebt dadurch den Fokus unnötig weg von der inneren Einstellung.

191 Ryan, „Embedded Narratives,“ 323. Siehe dazu oben, Kapitel 10, Abschnitt 3.

192 LN 30.56-74: „To Intend, To Purpose, To Plan.“

193 Siehe dazu oben, Kapitel 10, Abschnitt 3.

194 Siehe grundsätzlich oben, Kapitel 6, Abschnitt 3.4.1.2 für das Ipf. im Griechischen, wo das Deutsche eher den Ind. Präs. hat.

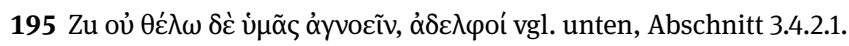

196 Für einen in der Vergangenheit lokalisierten, erfüllten Plan siehe Eph 1,9. In Röm 3,25 ist

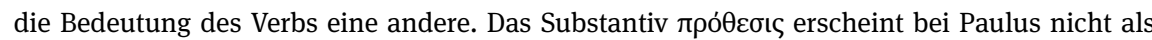
Einleitung eines durch ein Verb näher spezifizierten Vorsatzes. Zum „Umsetzen“ eines Vorsatzes im NT siehe Apg 27,13. 


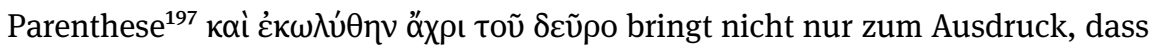

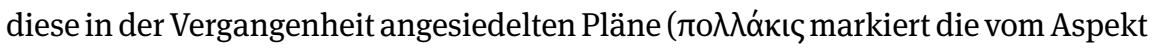
nicht ausgedrückte iterative Nuance) erfolglos blieben (,ich wurde gehindert“).

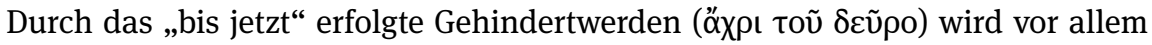
auch die Fortwirkung des Planes bis in die Gegenwart kommunziert (bzw. besser: seine Reaktivierung), ${ }^{198}$ dass also das „oft“ auch das ,jetzt“ miteinschließt.

Explizit gesagt wird dies dann in Röm 1,15, wo Paulus seinen Willen bekundet

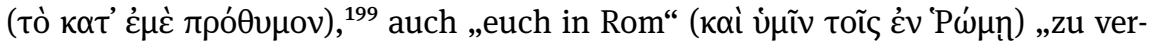

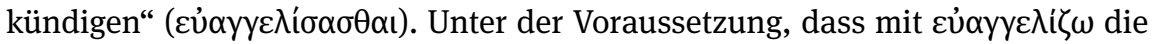
Missionspredigt gemeint ist und da es sich hier um einen Nominalsatz handelt

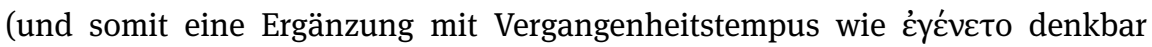
wäre), wurde öfter vorgeschlagen, dass hier ein ehemaliger - mittlerweile durch

197 Vgl. GGNT 292d.

198 Durch den perfektiven Aspekt wird die Aktserie als begrenzt dargestellt (und eventuell als eine konkrete Anzahl Planungs-Ereignisse konzeptualisiert; vgl. oben, Kapitel 6, Abschnitt 3.3.3.2 zu Armstrong, „Aorist“ und generell zur „boundedness“ von Situationen), aber nicht gesagt, dass in der realen Welt nicht auch noch in der Gegenwart (oder Zukunft) Ereignisse desselben Typs (siehe oben, Kapitel 3, Abschnitt 4.2 zur Frequenz) angesiedelt sein könnten. Vgl. dazu auch oben, Kapitel 6, Abschnitt 3.4.2.3 zum „Gedankenzusammenhange“ (KG 386), der das Fortbestehen in der Gegenwart impliziert. Vgl. allerdings Phil 2,12, das in der Tat zunächst schwierig

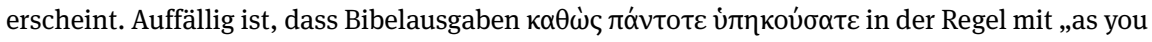

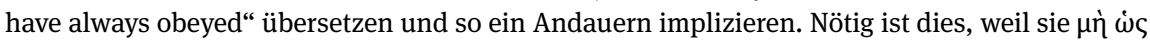

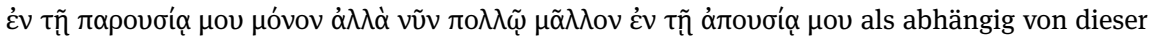
Situations-Schilderung betrachten. Die Situationszeit würde dann ein Zeitintervall umfassen, in welches sowohl die (je nach Datierung des Briefes) 5 bzw. 10 Jahre zurückliegende „Gegenwart“ als auch die gegenwärtige Abwesenheit - also das Jetzt des Briefschreibens - integriert sein müsste. Entsprechend kann die rechte Grenze der Situation aus Schreibersicht noch nicht erreicht worden sein. Paulus würde das Geschehen also sowohl als „,bounded“ als auch als „unbounded“ zugleich darstellen. Diese paradoxe Lesweise setzt etwa die Einheitsübersetzung ganz deutlich voraus: „Darum, meine Geliebten, - ihr wart ja immer gehorsam, nicht nur in meiner Gegenwart, sondern noch viel mehr jetzt in meiner Abwesenheit -: Wirkt mit Furcht und Zittern euer Heil!“

Eine ganz einfache Lösung ergibt sich, wenn man $\mu \eta \dot{~ . . . ~} \alpha \lambda \lambda \dot{\alpha}$... als Angaben zum Imperativ auffasst. Paulus würde dann zunächst von einem allzeitigen Gehorsam erzählen. (Nach

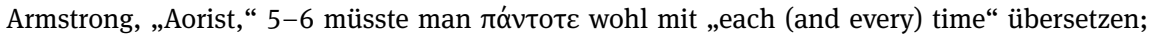
vgl. oben, Kapitel 6, Abschnitt 3.3.3.2.) Im Anschluss würde er dazu auffordern, (in Zukunft) auf das Heil mit Furcht und Zittern hinzuwirken. Dies sollen die Philipper auf eine bestimmte Weise tun: nicht nur, wie sie es ja auch in der Vergangenheit bereits taten (das „Wirken“ '̇v $\tau \tilde{n}$

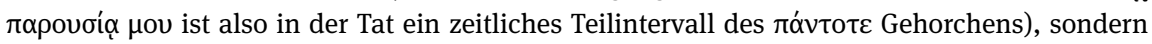
„nun“ (also im Jetzt, in welchem sie den Befehl erhalten, während Paulus nicht zugegen ist und nicht persönlich den Fortschritt überwachen kann) sogar noch mit vermehrten Anstrengungen.

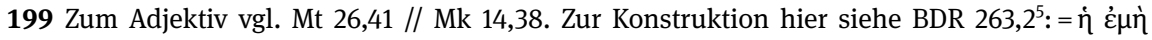

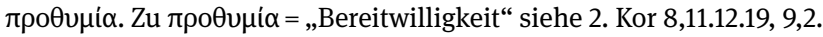


die Bekehrung der Römer überholter - Plan bezeichnet wäre. Das wäre zwar freilich mit dem schon „viele Jahre“ anhaltenden Verlangen nach Rom zu kommen

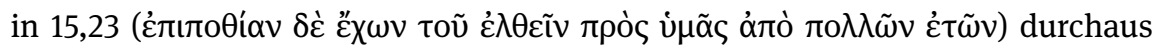
vereinbar, ignoriert aber den in jenem Vers genannten ausschlaggebenden

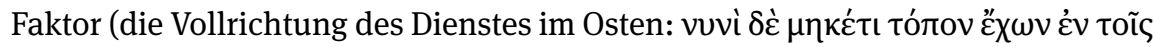

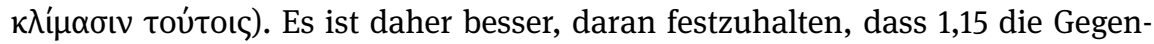

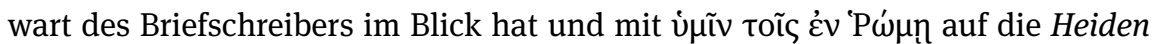
Bezug nimmt. ${ }^{200}$ Diese Auflösung legt sich nahe, wenn man die häufige Absicht in 1,13, nach Rom zu kommen, angemessen berücksichtigt: Dort ist nämlich durch einen Ǐvo-Satz der Zweck dieser Pläne spezifiziert: „damit ich auch unter euch eine gewisse Frucht haben möge, wie unter den anderen Heiden“ (İv $\alpha$ tı $\alpha$

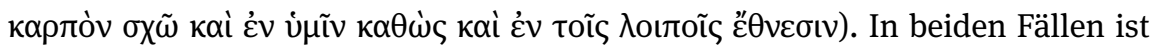
also wohl schlicht an ,in Rom“ als Kontext der Verkündigung gedacht.

Auffällig ist freilich, dass in 15,24 nur das „Genießen“ der Glaubensge-

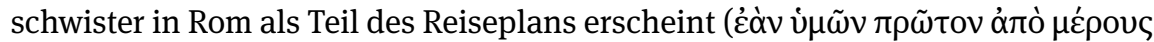
$\dot{\varepsilon} \mu \pi \lambda \eta \sigma \theta \tilde{\omega})$ und keine Evangelisationstätigkeit erwähnt wird. Auch in Röm 15,29,

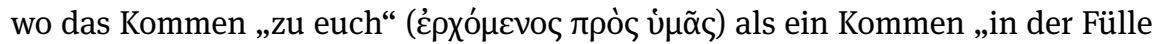

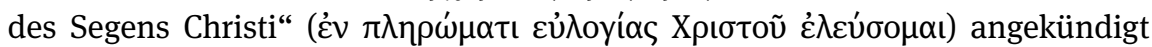
wird, scheint vor allem die Gemeinschaft mit den Christen in Rom im Blick zu sein. Geht man davon aus, dass Paulus in Röm 1,13 und 15 auf denselben Plan verweist (mit „Frucht“ in V. 13 also die erfolgreiche Verkündigung aus V. 15 gemeint ist und nicht etwa die in 1,11 angesprochene Mitteilung der Gnadengaben) und dass dieser zum Zeitpunkt des Schreibens noch aktuell ist, so entsteht freilich auch eine gewisse Spannung zum in Röm 15,20 angezeigten Grundsatz

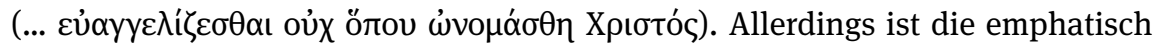
betonte Bereitwilligkeit in 1,15 kaum erklärbar, wenn man darin lediglich eine Aussage über die Vergangenheit sieht. ${ }^{201}$ Während man das Präsens in 1,14 noch anders erklären könnte, ${ }^{202}$ scheint außerdem das Präsens in 1,16 dafür zu sprechen, dass 1,15 an eine gegenwärtige Bereitschaft gedacht ist: Paulus wäre gerne bereit, in Rom zu verkündigen, weil er sich des Evangeliums nicht schämt (Oủ

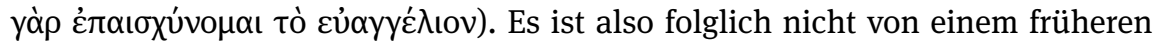
(Röm 1,13.15) Plan einer Evangelisationsreise nach Rom und einem späteren ,innerkirchlichen“ (Röm 15,24.29) Besuch auszugehen (oder 1,13 zu 1,11 zu ziehen und

200 So richtig Wolter, Brief, 114.

201 Zum einen könnte man dann fragen, ob denn dann zu folgern sei, dass Paulus nicht mehr bereit sei, in Rom das Evangelium zu verkündigen (vgl. Wolter, Brief I, 113), zum anderen würde diese aufwändige Rückblende dann sehr abrupt unterbrochen; es bliebe die Frage: „Und warum kamst Du dann nie?“

202 Gegen Wolter, Brief I, 113. 
so von 1,15 zu trennen), sondern lediglich von unterschiedlichen Schwerpunktsetzungen in den beiden (Proto-)Erzählungen. Ebenso erklärt sich auch die unterschiedliche Fokussierung auf die Erfahrung der Römer in 1,11 einerseits (,damit ich euch etwas an geistlicher Gnadengabe zu eurer Stärkung vermittle“; ǐv $\alpha \mathrm{\tau}$

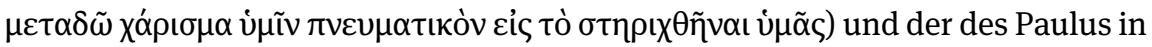
15,24 (wobei hier in 15,29 der Gedanke von 1,11 zumindest anklingt).

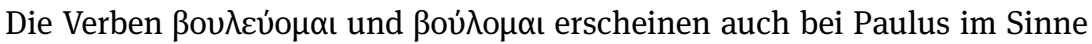
des Planens. ${ }^{203}$ In 2. Kor 1,15-17 wird der Doppelbesuch zunächst in V. 15 als

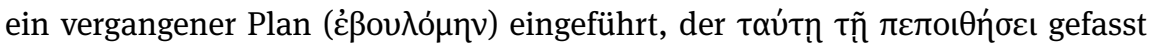
worden sei, also mit oder wegen besagter Zuversicht. In V. 17 nimmt das Partizip ßou入ó $\mu \varepsilon v o \varsigma$ nochmals auf diese Intention Bezug und geht der Frage nach, ob es sich hierbei um ein leichtfertiges Verhalten gehandelt habe - wobei auch hier wieder durch den Indikativ Aorist ein Bezug auf das vergangene Planen

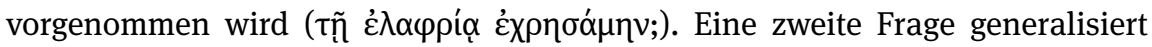
das Thema durch den Wechsel ins Präsens, wobei der ebenfalls stattfindende Wechsel des Verbs wohl lediglich stilistische Gründe hat: ${ }^{204}$ „[Oder] nehme ich

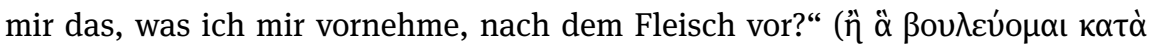

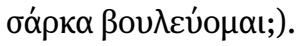

\subsection{Befürchtungssätze}

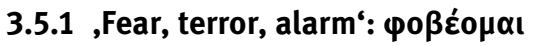

Hauptsätze mit prohibitivem Konjunktiv (Aorist) in der 2. und 3. Person ${ }^{205}$ mit $\mu \eta$ ' wurden schon früh in der griechischen Sprachgeschichte ,als abhängige verneinte Absichtserklärungen und als Befürchtungssätze empfunden,“206 sodass $\mu$ ' zur subordinierenden Konjunktion wurde. An Verben des Fürchtens, die mit abhängigem Befürchtungssatz ${ }^{207}$ konstruiert werden können, erscheint in den Paulusbriefen ${ }^{208}$ lediglich $\varphi$ oßźo $\mu \alpha$ und auch dieses nur 9 Mal.

203 LN 30.56: ,to think, with the purpose of planning or deciding on a course of action. ' Für

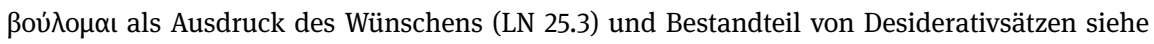
unten, Abschnitt 4.3.2.

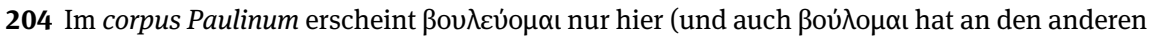
Stellen nicht dieselbe Bedeutung wie hier). Vgl. im NT Luk 14,31, Joh 11,53, 12,10, Apg 27,39.

205 Siehe dazu oben, Abschnitt 4.2.1.

206 Schwyzer-Debrunner II, 674.

207 Formal gehört der abhängige Befürchtungssatz zur Kategorie der abhängigen Behauptungssätze. Siehe GGNT 271e.

208 Siehe LN 25.251-269: „Fear, Terror, Alarm.“ Zur Terminologie in den Evangelien siehe

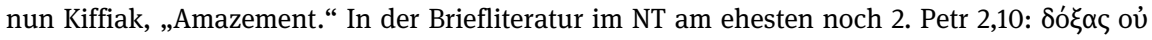


Teilweise wird auch die Konstruktion nach Verben wie $\beta \lambda \varepsilon ́ \pi \varepsilon \tau \varepsilon$ im Kontext von Befürchtungssätzen angeführt. ${ }^{209}$ Das betrifft dann etwa 1. Kor 8,9 ( $\beta \lambda \varepsilon ́ \pi \varepsilon \tau \varepsilon$

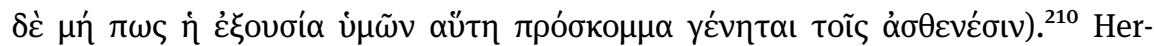
kömmlich wird hierin jedoch ein prohibitiver Konjunktiv in einem selbstständigen Begehrsatz gesehen, der asyndetisch an einen Imperativ angeschlossen ist („Achtung! ... !“). ${ }^{211}$ Dann wäre die Konstruktion eher unten (Abschnitt 4.2) im Rahmen befohlener und verbotener Handlungen $\mathrm{zu}$ diskutieren.

Eingewendet wird zuweilen, dass die in Frage stehende Konstruktion in 1. Kor 16,10 mit ǐv $\alpha$ vorliegt, womit sich die Frage aufdränge, ob nicht auch die anderen Konstruktionen als abhängige Sätze aufzufassen seien. ${ }^{212}$ Allerdings ist hier wohl eine verbsemantische Erklärung angebracht, die 1. Kor 16,10 als Son-

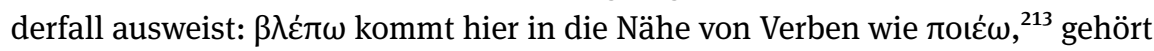
in dieser Bedeutung also zu den Verben des Bewirkens, welche der übergeordneten Kategorie der Verben des Begehrens und Wünschens zuzuordnen sind - die standardmäßig mit AcI, aber auch mit ìv $\alpha$-Satz stehen. ${ }^{214}$ Dementsprechend ist der Ǐv $\alpha$-Satz in 1. Kor 16,10 als abhängiger Begehrsatz aufzufassen.

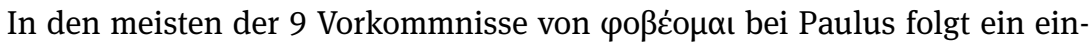
faches Akkusativobjekt, sodass ein etwaiger Ereignisbezug meist recht unklar bleibt. Dies gilt insbesondere, wenn es sich bei dem Gefürchteten um eine Person handelt. ${ }^{215}$

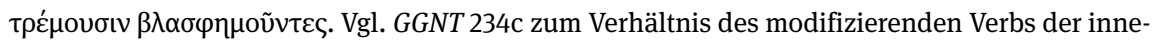
ren „Haltung der Subjektgröße“: Im Fokus steht das Lästern der Herrlichkeiten, welche durch das finite Verb näher bestimmt wird. Man könnte einerseits übersetzten als „sie lästern ohne Scheu“ oder auch als „,sie scheuen sich nicht ... zu lästern“ (NSS 1234). $\tau \rho \dot{\mu} \mu \omega$ würde im letzteren Fall dann auf gewisse Weise ein (nicht) „gefürchtetes“ Geschehen im Partizip einleiten. Nicht relevant sind die bei LN 25.223-250 aufgeführten Verben („Worry, Anxiety, Distress“), von denen keine Befürchtungssätze abhängig sind. Der Sache nach kann diese Terminologie allerdings natürlich durchaus auf Sorgen des Apostels Bezug nehmen. Vgl. etwa „die Sorge um alle Gemein-

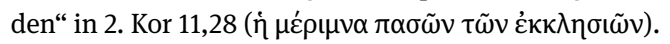

209 So Wallace, Grammar, 477: ,watching out for.“

210 Mit Indikativ Futur statt Konjunktiv in Kol 2,8.

211 So sowohl BDR $364^{3}$ und GGNT 210e.

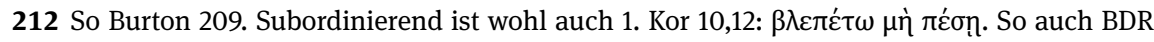
$462^{2}$.

213 LN 13.134: ,to take responsibility for causing something to happen.' Zu übersetzen wäre im Fall von 1. Kor 16,10 also mit „Sorgt dafür, dass ...“

214 GGNT 218ab.

215 Für Eph 5,33 (mit ỏvńp als Akkusativobjekt) und Kol 3,22 (mit kúpıoৎ als Akkusativobjekt) wird zudem in der Regel eine die Ehrfurcht ausdrückende Bedeutung angenommen. 
Im Römerbrief erscheint das Verb außer in 11,21 216 auch noch in Röm 13,3 und 4. In V. 3 folgt ihm schlicht der einfache Akkusativ: „Willst Du die Macht nicht

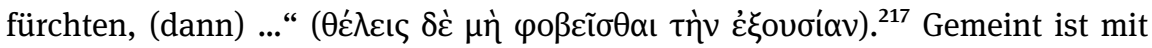
dieser Furcht, wie unten (Abschnitt 5.2.3.2) erläutert, genauer das Ereignis des von der staatlichen Autorität ausgehenden „Urteils,“ das in 13,2 erwähnt wird.

In Röm 13,4 erscheint - in einer vermeintlichen Parallele zur Konstruktion in 11,20-21 - wieder der Imperativ $\varphi$ oßov, diesmal mit (vorausgestelltem prospek-

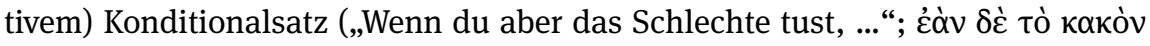
$\pi о$ п๊̃, $\varphi \circ \beta$ о̃). Der Inhalt dieses Fürchtens wird durch den nächsten Satz spezifiziert: das „Schwert“ ( $\mu \alpha \dot{\chi} \chi \alpha \iota \rho \alpha){ }^{218}$

Mit personalem direkten Objekt ist auch Gal 2,12 konstruiert. Hier begründet Paulus den Rückzug des Paulus von der Mahlsgemeinschaft mit den Heiden(chris-

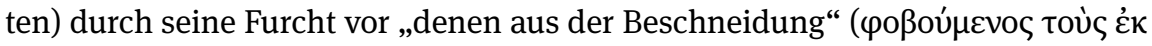

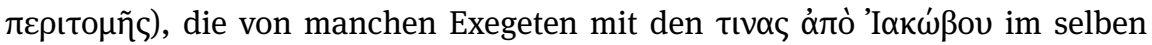
Vers gleichgesetzt werden (vorausgesetzt es ist nicht $\tau ı v \alpha$ zu lesen), von anderen auf jüdische Kreise bezogen werden, welche gerade auch für den Jerusalemer Kreis bedrohlich gewesen sein dürften. Entsprechend unterschiedlich hat man sich wohl auch den genauen Inhalt dieser unterstellten Furcht vorzustellen. ${ }^{219}$

Auch in der Formulierung von Gal 4,11220 folgt auf $\varphi \circ \beta$ ṽ $\mu \alpha$ z zwar zunächst ein Pronomen im Akkusativ (ن் $\mu \tilde{\alpha} \varsigma$ ), doch Paulus fürchtet natürlich nicht die Galater. Vielmehr liegt wohl eine Prolepse vor, ${ }^{221}$ wobei sich der Nebensatz in

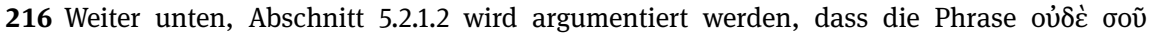

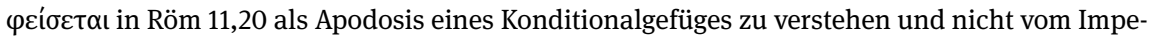
rativ $\varphi \circ \beta 0 \tilde{~ i n ~ 11,20 ~ a b h a ̈ n g i g ~ i s t . ~}$

217 Vgl. GGNT 331b. Zur Stelle siehe auch unten, Abschnitt 4.4.2 und Abschnitt 5.2.3.2.

218 Mit yó $\rho$ wird zudem eine Begründung mit Äußerungsbezug geliefert: Die Aufforderung zur

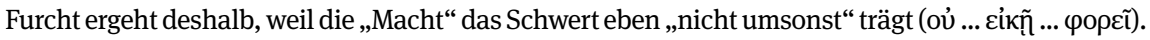
219 Im Kontext dient das Motiv der Furcht vor allem dazu, das Verhalten des Petrus als nicht seiner eigentlichen Überzeugung entsprechend zu kennzeichnen. Im Hinblick auf die Situation in Galatien ergibt sich durch den Finalsatz in Gal 6,12, wonach die Unruhestifter die Beschneidung in den galatischen Gemeinden durchsetzen wollen, „nur damit sie nicht wegen des Kreu-

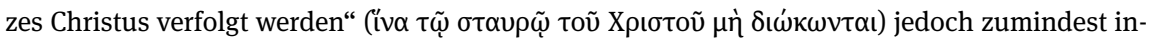
direkt eine Fokussierung auf das Motiv der Furcht. Abhängig davon, wie man die Erzählung des Paulus in Gal 2,11-21 versteht (siehe zu den verschiedenen „Verständnisweisen“ oben, Kapitel 5, Abschnitt 5.2.2), wird man daher den - hier natürlich ebenfalls recht offen bleibenden - Inhalt der Furcht auch in Gal 2,12 eintragen dürfen.

220 Vgl. zum literarischen Kontext oben, Kapitel 9, Abschnitt 5.

221 BDR $477^{4}$. Allerdings wird hier nicht wirklich etwas aus der abhängigen Konstruktion ,vor-

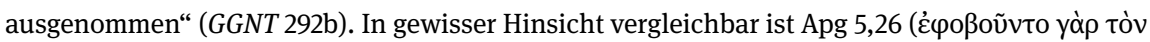

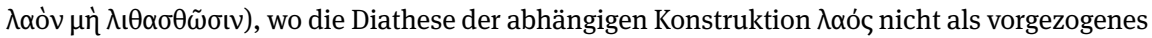

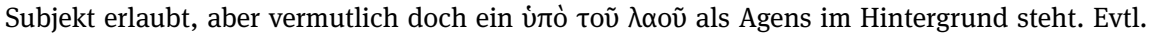


diesem Fall auf die Vergangenheit bezieht, was durch den Indikativ angezeigt wird. ${ }^{222}$ Damit bleiben nur zwei Stellen im corpus Paulinum, in denen eine Furcht des Paulus durch $\varphi$ oßoũ $\mu \alpha \mathrm{t}+\mu \eta \dot{n} \pi \omega \varsigma^{223}+$ Konjunktiv ausgedrückt wird. ${ }^{224}$

In 2. Kor 11,2 begründet Paulus sein präsentisch ausgedrücktes und im göttli-

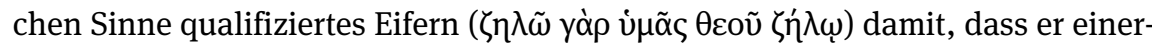
seits auf die durch ihn durchgeführte „Verlobung“ der Gemeinde zurückblickt

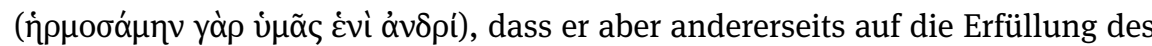
Zwecks dieser Verlobung vorausschaut, ${ }^{225}$ welcher darin besteht, die Korinther

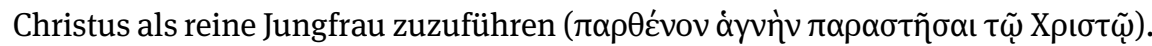

Es ist der durch dieses Bild aufgespannte zeitliche Rahmen, welcher das Präsens $\varphi$ ○оũ $\mu \alpha$ in 11,3 erklärt: Paulus sieht das durch den Infinitiv in 11,2 ausgedrückten Ziel gefährdet. Interessanterweise wählt Paulus für die Formulierung des Inhalts dieser Furcht kein Indikativ-Verb sondern den Konjunktiv: Er fürchte, „dass euer Sinn verführt werden könnte, weg von der auf Christus ausgerichte-

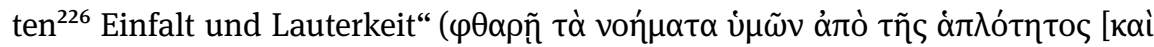

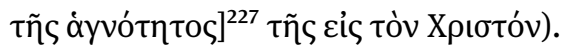

Dass die Befürchtung mit der Verführung Evas verglichen wird ( $\dot{\omega} \varsigma$ ò ő $\varphi$ เs

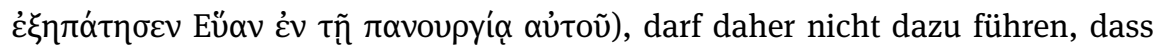
man sie gleichermaßen auf die Vergangenheit bezieht. Verlockend ist eine solche Deutung auch aufgrund des Folgeverses V. 4: Nimmt man an, dass das yó $\rho$ eine begründende Konnexion mit der Befürchtung in V. 3 eingeht und nicht auf die Aufforderung in V. 1 zurückverweist, ${ }^{228}$ so scheint die Sorge des Paulus hier in der Protasis bereits gegebenem Einfluss der Überapostel vorauszusetzen: „Wenn der Kommende $^{229}$ einen anderen Jesus verkündigt, den wir nicht verkündigt haben,

wäre es doch angemessener, hier von einem Akkusativ der Beziehung auszugehen (vgl. GGNT 154d), auch wenn dieser grundsätzlich in der Exegese inflationär postuliert wird (vgl. Heilig,

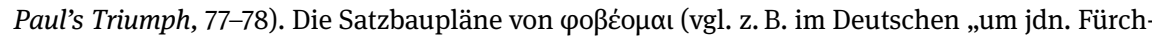
ten“) scheinen zumindest noch mehr Aufmerksamkeit zu verdienen.

222 Siehe daher zur Stelle oben, Kapitel 12, Abschnitt 7.2.

223 GGNT 247b verweist auf $\mu$ ń und ov̉ $\mu$ ń nach Ausdrücken des Fürchtens. Siehe jedoch auch Schwyzer-Debrunner II 674-675 zu $\mu$ '́ $\pi \omega \varsigma$.

224 GGNT 210h. Vgl. zum Satztyp auch 271e.

225 Siehe unten, Abschnitt 5.3 bei den finalen Konnexionen zu dieser Stelle.

226 Die Annahme von Stegman, Character, 196-202, dass عis tòv Xpıotóv auf das in Christus Bestehende Bezug nimmt, hat wenig Evidenz für sich.

227 Vgl. Kurek-Chomycz, „Sincerity“ zur Textkritik.

228 Vgl. Schmeller, Brief II, 203-204 für eine Übersicht über die Positionen. In der Regel wird dann das yó $\rho$ in V. 5 wieder gemeinschaftlich auf V. 1 bezogen (vgl. etwa Bultmann, Brief, 203 und 205 für einen Bezug auf V. 1 in beiden Fällen).

229 Der Bezug auf eine Einzelperson, etwa einen Schlichter aus Jerusalem, ist in der Exegese weitestgehend aufgegeben. Vgl. bereits Bultmann, Brief, 203. 
oder ihr einen anderen Geist empfangt, den ihr nicht empfangen habt, oder ein

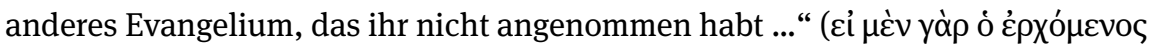

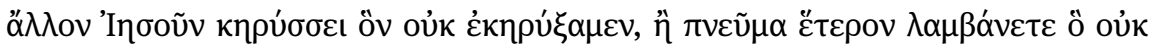

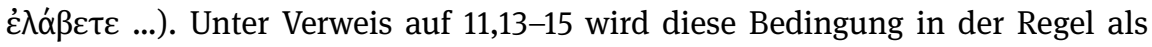
bereits erfüllt angesehen. Ob man in V. 4 aber in der Apodosis „... dann ertragt

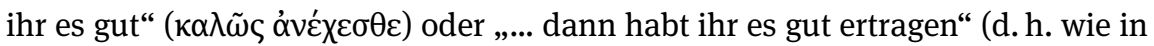
einem Teil der Überlieferung mit Augment) liest, ist vollkommen gleichgültig für die Beurteilung der Protasis - so oder so wird die Periode nicht zu einem irrealen Fall. ${ }^{230}$ Wie später in 11,20 wird man daher aus der Periode in 11,4 nicht viel darüber ableiten dürfen, was in Korinth zu diesem Zeitpunkt bereits geschehen ist. Vielmehr formuliert die Periode durch den Präsens in beiden Teilen wie auch sonst oft bei Paulus einen regelhaften Zusammenhang. ${ }^{231}$ Deswegen erscheint das substantivierte Partizip ó દ́pxó $\mu \varepsilon v o \varsigma$ hier auch ohne Indefinitpronomen (d. h.

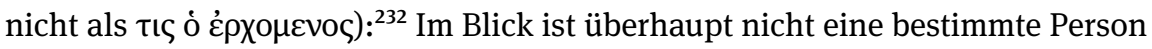
oder Gruppe an Personen, die aus rhetorischen Zwecken anonymisiert würde. Vielmehr drückt das Partizip den generellen Fall aus, ${ }^{233}$ dass jemand kommt (also

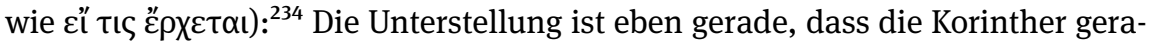
dezu automatisch einen jeden Kommenden bereitwillig ertragen, selbst wenn dieser sich noch so absurd verhält. ${ }^{235}$ Diese umfassende Aussage hat sicherlich einen Grund im bereits an den Tag gelegten Verhalten, im Kontext erfüllt sie jedoch vor allem die Funktion, die in V. 3 auf die Zukunft gerichtete Sorge des Paulus zu begründen.

230 Das öv kann zwar im NT ausfallen (GGNT 284a), doch das ändert absolut nichts am fehlen-

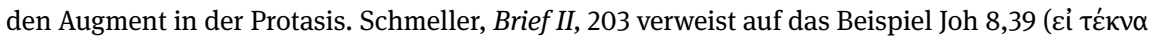

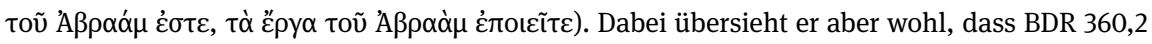
hier die Variante mit $\tilde{\eta} \tau \varepsilon$ für $\dot{\varepsilon} \sigma \tau \varepsilon$ anführen. Folgt man dem Standardtext, dann liegt auch dort keine irreale Konditionalperiode vor, sondern eine Mischform (GGNT 285a; zur Stelle vgl. auch NSS 1025-1026). Hier ist aufgrund der indikativischen Präsens-Formen aber ohnehin von einem prototypischen indefiniten Fall zu sprechen. Vgl. demgegenüber die Variante $\alpha v \varepsilon ı \chi \varepsilon \sigma \theta \varepsilon$ (vgl. kurz zuvor - als Irrealis - in 2. Kor 11,1; vgl. zur Stelle oben, Kapitel 12, Abschnitt 8.4).

231 Vgl. etwa Röm 7,16-20, 8,25, 1. Kor 14,38, Gal 5,18. In 1. Kor 9,17 ist die Sache etwas anders gelagert, weil das präsentische „Lohnhaben“ eine futurische Referenz hat. Vgl. unten, Abschnitt 5.2.1.1.

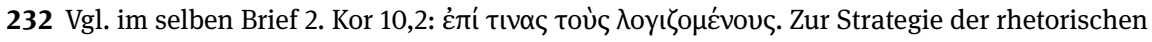

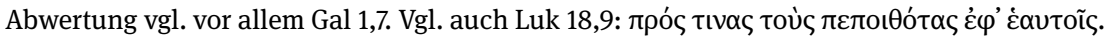

233 Vgl. zum so gebrauchten substantivierten Partizip im Konditionalsatz (mit prospektivem Fall) 1. Sam 11,3: ćàv $\mu$ ì

234 Vgl. Luk 14,26 und 2. Joh 10.

235 Ein inhaltlich sehr ähnlich gelagerter Fall wird in 1. Tim 6,3-4 durch eine indefinite Konditionalperiode ausgedrückt: Hier steht im Konditionalsatz тıৎ für die falsch verkündende Person. Die Apodosis in 6,4 ist hier durch den Indikativ Perfekt ausgedrückt, da hier das Wesen des sich so Verhaltenden bewertet und nicht das Verhalten der „Empfangenden“ beschrieben werden soll. 
In 2. Kor 12,20 sind auch allerlei exegetische Fragen $\mathrm{zu}$ klären, die den genauen Inhalt des Befürchteten betreffen. Allerdings ist der futurische Bezug hier aufgrund des Kontextes völlig unstrittig. So wird der Zeitpunkt, auf den sich die Befürchtung bezieht, durch das temporale Partizip غ่ $\lambda \theta \dot{\omega} v$ markiert: „wenn ich komme ..."

Das Befürchtete wird dann recht umfassend geschildert: „... dass ich euch nicht in dem Zustand finde, wie ich es wünsche, und ich nicht von euch so gefun-

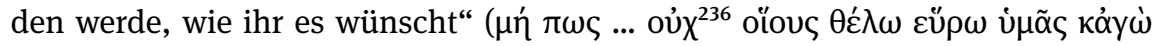

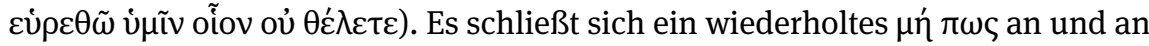
dieses knüpft ein das Moment der Konfrontation hervorhebender Lasterkatalog an: „... dass (es geben wird) Streit, Eifersucht, Wutausbrüche, Rivalitäten, Verleumdungen, Geläster, arrogantes Verhalten, Durcheinander ( $\mu \eta \dot{\prime} \pi \omega \varsigma$ [erg. $\tilde{\omega} \sigma \mathrm{t}]^{237}$ ع̋ Auch V. 21 führt den Inhalt der Befürchtungen fort, wobei diesmal einfach $\mu$ ń verwendet wird. Erneut wird durch ein temporal verwendetes Partizip - diesmal als

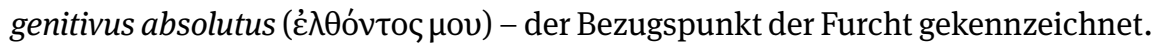

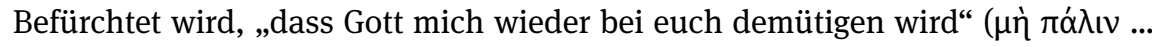

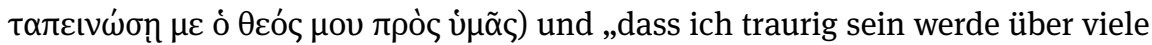
der Zuvor-gesündigt-und-nicht-Buße-getan-Habenden wegen der Unreinheit und

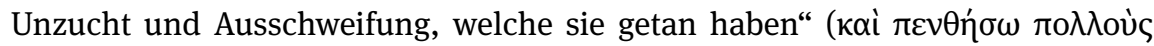
$\tau \tilde{\omega} \nu \pi \rho{ }$

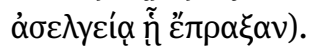

Auffällig ist hier zum einen, dass das $\pi \alpha ́ \lambda ı v$ - sofern man es nicht mit dem Partizip verbindet ${ }^{239}$ - indirekt nun auch den Zwischenbesuch als „Demütigung“ charakterisiert. ${ }^{240}$ Was das befürchtete Geschehen angeht, ist die Konstruktion in

236 Es steht hier nicht $\mu$ ń vor dem Konjunktiv, um die Verwechslung des Partikels als Konjunktion auszuschließen. Siehe Robertson, Grammar, 1161.

237 Vgl. NSS 1032.

238 Zum Plural vgl. GGNT S. 182. Teilweise wird die These vertreten, die ersten beiden Bestandteile, die im Singular auftreten, bezeichneten ,allgemein das Gebiet der Verfehlungen, das dann durch sechs Glieder im Plural illustriert wird“ (Schmeller, Brief II, 357). Aus dem Numerus wird man das allerdings nicht ableiten dürfen. Siehe etwa Gal 5,20, wo beide Lexeme ebenfalls im

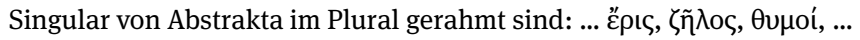

239 Und dagegen sprechen gute Gründe. Siehe Schmeller, Brief II, 359-360.

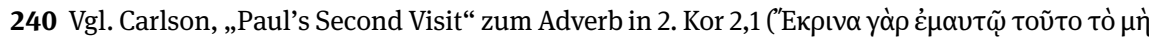

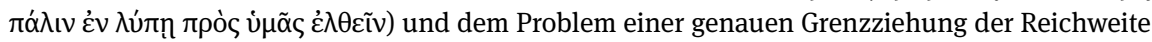
des Adverbs. Dasselbe Problem stellt sich freilich hier, wobei man aber zumindest argumentieren kann, dass von V. 20 her kommend, eine Spezifierung des Wiederkommens nicht nötig erscheint,

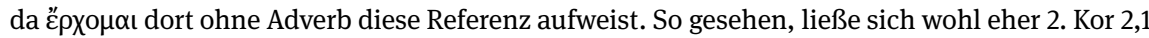
vor dem Hintergrund von 2. Kor 12,20-21 entsprechend der Mehrheitsmeinung auslegen (wobei 
zweifacher Hinsicht von Bedeutung: Erstens kann man fragen, weshalb Paulus lediglich befürchtet ,viele“ der durch die Genitive gekennzeichneten Gruppe betrauern zu müssen. Zweitens kann man diskutieren, welchen zeitlichen Bezug die substantivierten Partizipien haben. Semantisch beinhaltet $\pi \rho \alpha \mu \alpha \rho \tau \alpha \dot{v} \omega$ bereits einen Vergangenheitsbezug. Es erscheint außer in 2. Kor 12,21 nur noch in 2. Kor 13,2 und auch dort als Perfektpartizip. ${ }^{241}$ Die Vermutung liegt nahe, dass Paulus hiermit auf ein früheres Stadium seiner Interaktion Bezug nimmt, also auf diejenigen, die sich bereits beim Zwischenbesuch als „Sünder“ erwiesen hatten. In der Regel wird das den Akt der Buße betreffende Aorist-Partizip dann auf eine ausbleibende Umkehr in der gesamten sich anschließenden, bis zum angekündigten Besuch reichenden Zeitspanne bezogen.

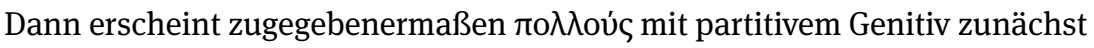
„sinnlos“242 - zumindest, wenn man annimmt, dass aus einer solchen Datierung folgt, dass die Trauer in einem solchen Fall nur einem Teil der bis zum Eintreffen des Paulus uneinsichtigen Sünder gelten würde. Die diesem Urteil zugrundelie-

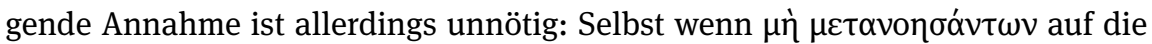
Zeit nach dem Zwischenbesuch Bezug nehmen würde, dann wird man doch wohl zumindest einige von ihnen den laut 2. Kor 7,9-10 in Reaktion auf den Tränenbrief

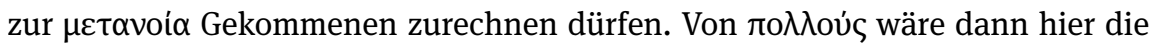
Rede, weil Paulus die Möglichkeit erwägen würde, dass einige der zum Zeitpunkt des Verfassens des Tränenbriefes noch Uneinsichtigen (d. h. nicht aufgrund des Zwischenbesuches Umgekehrten) noch immer in ihrem Sündersein verharren

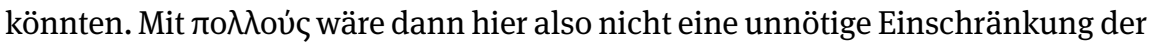
Trauer vorgenommen, sondern vielmehr eine erstaunliche Verschärfung gegenüber 2. Kor 7,9-12 ausgedrückt, wo die Reaktion der Gemeinde als Ganzer auf den Tränenbrief noch sehr positiv dargestellt wurde.

Es bietet sich allerdings noch eine weitere Deutungsoption der temporalen Beziehung an. Denn es wäre von der Grammatik her auch ohne Weites möglich, das Unterlassen der Umkehr noch weiter zurückzudatieren: Die Unterscheidung im Stamm der Partizipien impliziert schließlich keine Zeitenfolge ${ }^{243}$ und

natürlich dabei die Integrität des Briefes vorausgesetzt werden müsste). Carlson (persönliche Mitteilung) würde das Adverb in 2. Kor 12,21 analog zu seiner Diskussion in Carlson, „Polysemy,“ 65-68 als „reframing“ einordnen: Es zeige lediglich an, dass das Thema des Befürchtens wieder aufgegriffen werde. Paraphrasierend wäre also wohl zu übersetzen: „Ich befürchte wiederum (=außerdem), dass ....“

241 Die passive Form für „frühere Verfehlungen“ ist bei Josephus, B. J. 1.481 belegt.

242 Bultmann, Brief, 241.

243 Kontextuell leicher erschließbar ist diese in 2. Kön 23,17, wo eine erstaunlich ähnliche Konstruktion (Perf. Ptz. + Aor. Ptz. + Rel.-Satz mit Ind. Aor.) vorliegt: „Dies ist der Mann, der aus Judäa 
es wäre durchaus denkbar, dass Paulus mit den beiden Partizipien eine Gruppe umschreibt, die er beim Zwischenbesuch in exakt dieser doppelten Verfassung antrifft: zum damaligen Zeitpunkt uneinsichtige Sünder. Das Aorist Partizip des (unterlassenen) Umkehrens könnte sich also ebenso auf diese vorausgehende Zeit beziehen, wie der zum Zustand des Sünderseins führende Akt, welcher im selben

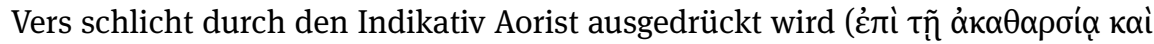

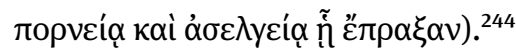

Dafür spricht nun vor allem das $\pi \dot{\alpha} \lambda \iota v$, das, wie bereits erwähnt, verwundern muss, wenn man es nicht auf das Partizip bezieht (also im Sinn von: „wenn ich erneut komme"): Die für die Zukunft befürchtete Demütigung ist dieselbe, wie die einst schon erlittene, als der Gemeindegründer beim erneuten Besuch erkennen musste, dass die vormals von ihm Bekehrten noch immer an ihren alten Sünden festhielten. ${ }^{245}$

\subsubsection{Ohne Ausdrücke des Fürchtens}

An einigen Stellen ist bei Paulus ein Ausdruck des Fürchtens zu ergänzen, da sich die den abhängigen Befürchtungssatz einleitende Konjunktion „an irgendein Verbum anhäng[t], um das begleitende und bestimmende Gefühl der Besorgnis auszudrücken. “246 Einige dieser Fälle werden im Abschnitt zu auf die Vergangenheit bezogenen Befürchtungen besprochen (siehe Kapitel 12, Abschnitt $7.2 \mathrm{zu}$ Gal 2,2 und 1. Thess 3,5).

An dieser Stelle ist jedoch darauf hinzuweisen, dass es bei auf die Zukunft bezogenen Befürchtungen, die ja durch den Konjunktiv ausgedrückt werden, aufgrund des fehlenden lexikalischen Hinweises leicht zu Abgrenzungsproblemen mit Finalsätzen kommen kann: Denn in diesen kann statt ĩvo $\mu$ auch lediglich $\mu \eta ́$ (mit anschließendem Konjunktiv) stehen. ${ }^{247}$

Fehlt der Ausdruck des Befürchtens, dann wird manchmal - auch schon klassisch (vgl. etwa Platon, Gorg. 462e) - auch lediglich eine Vermutung oder

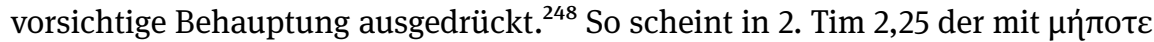

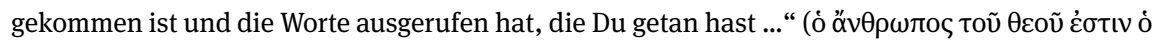

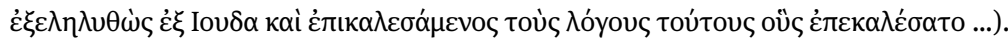

244 Zum Attribut siehe GGNT 265c.

245 In alternativen Auslegungen bleibt das $\pi \alpha \dot{\lambda} ı v$ immer nur teilweise angebracht. Vgl. Schmeller, Brief II, 359-360.

246 BDR 270,2. Vgl. GGNT 247b. Zu $\mu$ ń $\pi \omega$ ç in Röm 11,21 siehe ausführlich unten, Abschnitt 5.2.1.2. 247 GGNT 278a. Vgl. etwa 1. Kor 9,27. Siehe auch GGNT 335 zur modal-instrumentalen Konnexion mit dem dortigen Verweis auf 2. Kor 2,7. Kein Problem ergibt sich freilich, wenn stattdessen ein finaler Infinitiv (GGNT 210) verwendet wird.

248 Vgl. CGCG 34.10: „... to express an anxious, cautious or tentative assertion.“ 
eingeleitete Satz generell eine positive - aber eben unsichere - Entwicklung in den

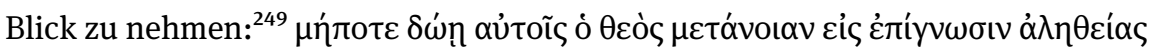
wäre dann zu übersetzen als ,Vielleicht wird Gott ihnen Buße zur Erkenntnis der Wahrheit geben. ${ }^{\text {250 }}$ Funktionell sind solche Konstruktionen daher Behauptungssätzen mit Indikativ Futur, die durch ľowৎ etc. abgeschwächt sind, nicht unähnlich. ${ }^{251}$

\section{Begehrsätze}

\subsection{Hinführung}

Zukünftiges Geschehen spielt auch noch in einer weiteren großen Kategorie eine Rolle - im Hinblick auf Ereignisse, deren Eintreten dadurch als unsicher markiert wird, dass es überhaupt erst vom Sprecher als $z u$ tun thematisiert wird. Die Ereignisse, auf welche auf diese Weise Bezug genommen wird, werden so als vom unausweichlichen Lauf der Dinge abgehoben gekennzeichnet: Es muss aktiv gehandelt werden, um sie zu verwirklichen. ${ }^{252}$ Damit ist zugleich auch klar, dass diese Ereignisse im Regelfall gerade nicht in der passiven Zukunftsprognose enthalten sind. ${ }^{253}$

Es wurde am Beispiel von Gal 4,12 $2^{254}$ bereits illustriert, wie die Aufforderung zu einer Handlung einen Knotenpunkt innerhalb eines größeren narrativen Ereigniszusammenhangs darstellen kann, von welchem aus verschiedene Handlungsstränge möglich sind. Doch auch wenn ein zukünftiges Ereignis isoliert als erwünscht charakterisiert wird, liegt hierin bereits das Potenzial für eine minimale Protoerzählung, da eine solche Äußerung immer auch in Bezug zum

249 Burton 225 rekonstruiert mit einigem Umweg eine tatsächliche Sorge: Die Zurechtweisung solle in Sanftmut erfolgen, weil andernfalls die Möglichkeit besteht, dass Gott den besagten Personen in seiner Gnade die Umkehr schenke und man selbst sich dadurch etwas zu Schulden hätte kommen lassen, dass man denen, denen Gott mit Gnade begegnet, mir Härte statt Sanftmut entgegen getreten wäre.

250 So etwa die NET: „Perhaps God will grant them repentance and then knowledge of the truth.“ Weiser, Brief, 235 spricht ähnlich vom „Ziel, zu dem die Zurechtweisung die Irrlehrer und die von ihnen Verführten bringen soll,“ versäumt es jedoch, diese Auslegung in der Syntax zu verankern. Siehe auch GGNT 247d.

251 Siehe dazu oben, Abschnitt 3.3.3.

252 Vgl. zu Lämmerts Rede von der „Zukunftsgestaltung“ oben, Kapitel 11, Abschnitt 4.

253 Vgl. oben, Kapitel 10, Abschnitt 3 zu Ryans eingebetteten mentalen Narrativen.

254 Kapitel 9, Abschnitt 5.4.4; vgl. auch zu Röm 12,19 in Kapitel 13, Abschnitt 2.2.4 und unten, Abschnitt 5.2.3.2 zu Röm 13,3-4. 
Ist-Zustand auf der einen Seite und dem Resultat der passiven Prognose auf der anderen Seite steht. In der großen Mehrheit der Fälle wird in den Texten jedoch auf diese anderen Bezugspunkte zumindest andeutungsweise Bezug genommen (siehe etwa unten, Abschnitt 4.2.2 zu Röm 6,13.19), was die Identifikation von potenziellen Erzählungen sehr erleichtert.

Ereignisse, welche die Zukunftsgestaltung betreffen, können in Texten auf sehr verschiedene Weisen ausgedrückt werden, da die „Pläne, Entschlüsse, Anordnungen und Verabredungen“ “255 von Lämmerts Liste nicht auf bestimmte syntaktische Konstruktionen oder Satztypen festgelegt sind. An dieser Stelle kann daher nur auf einige wenige Optionen hingewiesen werden, die jedoch einen großen Teil der relevanten Texte abdecken. Die Pragmatik des Textes kann selbstverständlich auch oft auf Motivation zum Handeln ausgerichtet sein, wenn der Text ausschließlich aus Aussagesätzen besteht (vgl. „Es ist laut hier drinnen“ - kann in manchen Kontexten etwa eine sogenannte indirekte Sprechhandlung sein, wobei sich hinter der augenscheinlichen Feststellung eigentlich der illokutionäre Akt einer Bitte oder Rüge verbirgt). ${ }^{256}$

\subsection{Selbstständige Begehrsätze: Aufforderungssätze}

\subsubsection{Hinführung}

Dabei sind natürlich in erster Linie die selbstständigen Begehrsätze zu nennen. ${ }^{257}$ In der großen Mehrheit der Fälle bringen sie Aufforderungen zum Ausdruck, die entweder an den Sprecher selbst (in der 1. Person Singular), an die Angesprochenen (in der 2. Person Singular/Plural), an beide (1. Person Plural), oder Dritte gerichtet sind (3. Person Singular/Plural). Dabei tauchen die Verben entweder im Imperativ (2./3. Person) auf oder erscheinen im voluntativen Konjunktiv als adhortativer Konjunktiv für die 1. Person ${ }^{258}$ oder als prohibitiver Konjunktiv $^{259}$ als alternative Ausdrucksmöglichkeit der negativen Aufforderung, also des Verbots.

255 Lämmert, Bauformen, 177.

256 Siehe grundsätzlich CGCG 38.1-3 für die Differenzierung zwischen „sentence types and communicative functions “ mit Beispielen des Griechischen. Vgl. bei Paulus etwa die Feststellung in Gal 4,31, die im Kontext klar appellativen Charakter hat. Siehe oben, Kapitel 8, Abschnitt 4.4.4. 257 Für eine Übersicht über die selbstständigen Begehrsätze siehe GGNT 268ab.

258 GGNT 210c.

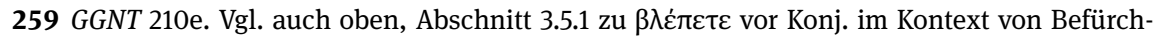
tungssätzen. 
Neben diesen den Imperativ ergänzenden Ausdrucksmöglichkeiten, gibt es in der Koine auch einige Ersatzkonstruktionen für den Imperativ, ${ }^{260}$ die hier aus Platzgründen nur ganz knapp angerissen werden können:

(1) $\mathrm{Zu}$ nennen ist hierbei natürlich der verneinte Indikativ Futur zum Ausdruck eines strikten Verbots, ${ }^{261}$ wie es etwa im Zitat „Du sollst nicht begehren!“ aus Ex 20,17/Dtn 5,21 in Röm 7,7 vorliegt. Dasselbe gilt für die Ex 20,13-15, 17, Dtn 5,17-19.21 und Lev 19,18 entnommenen Verbformen in Röm 13,9.

(2) Wie in der petrinischen Briefliteratur taucht auch bei Paulus - etwa in Röm 12,9-19 - das Partizip gelegentlich imperativisch auf. ${ }^{262}$

(3) Ebenso anzutreffen ist an manchen Stellen in den paulinischen Briefen (1. Kor 7,29, 2. Kor 8,7, Gal 2,10, Eph 5,33) ein imperativischer Konjunktiv nach selbstständigem ǐvo, welcher die spätere standardmäßige Konstruktion mit vo antizipiert. ${ }^{263}$

(4) Der Gebrauch des Infinitivs zur Formulierung einer Aufforderung hat sich im Attischen gegenüber der Dichtung Homers bereits deutlich zurückgebildet und erscheint im NT lediglich zwei Mal, wobei beide Vorkommnisse paulinisch sind und eines im Römerbrief vorliegt (Phil 3,16 und Röm 12,15). ${ }^{264}$

Im Folgenden sollen nun jeweils an einem Beispiel die beiden relevantesten Kategorien - der Imperativ und der voluntative Konjunktiv - besprochen werden.

\subsubsection{Der durative Imperativ in Röm 6,13}

Im Römerbrief drückt Paulus 62 Mal eine Aufforderung durch einen Imperativ aus - wobei allerdings mehr als 25\% (nämlich 16 Vorkommnisse) auf die Auffor-

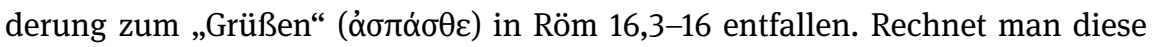
heraus, tritt auch die für Paulus typische Bevorzugung des durativen Imperativs deutlicher hervor (34:12 statt 34:28). ${ }^{265}$

260 Für einen Überblick siehe GGNT 212g. Auch das modal gebrauchte Futur kann einige dieser Funktionen übernehmen, wurde jedoch oben, Abschnitt 3.1 bereits besprochen und soll daher nicht weiter in die Diskussion mit aufgenommen werden.

261 GGNT 202f.

262 GGNT 231j. Die Verwendungsweise fällt unter den prädikativen Gebrauch, da hier von einer

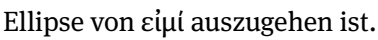

263 Vgl. jedoch die sprachgeschichtlich ebenfalls naheliegende Einordnung von Caragounis, Development, 226 zu 1. Kor 7,29. Zur Konstruktion mit va und der Vorläuferform im NT siehe Caragounis, Development, 220.

264 GGNT 218d.

265 Dieser „korrigierte“ Anteil von ca. 74\% entspricht ziemlich genau dem auf den gesamten corpus Paulinum bezogenen Wert von ca. 77\%: Auf 425 Imperative kommen hier nur 97 im AoristStamm und 327 im Durativ-Stamm (sowie in Eph 5,5 der resultative Imperativ l̈otع vom der Bedeutung nach präsentischen oî $\delta \alpha)$. 
Da im Zusammenhang mit Gal 4,12 (Kapitel 9, Abschnitt 5.4.4) bereits ausführlich auf die Auswirkungen von Imperativ-Formen auf implizite Handlungsstränge hingewiesen wurde, soll hier ein einziges weiteres Beispiel für diese Kategorie genügen.

Bei diesem Imperativ aus Röm 6,13 handelt es sich um das erste Auftreten der unmarkierten ${ }^{266}$ Aorist-Form eines Imperativs im Römerbrief. Hier werden die

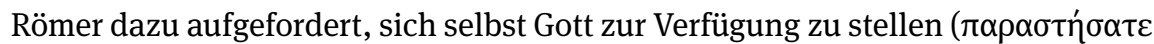
$\dot{\varepsilon} \alpha u \tau o u ่ \varsigma ~ \tau \tilde{\omega} \theta \varepsilon(\tilde{\omega})$. Dieselbe Form von $\pi \alpha \rho i ́ \sigma \tau \eta \mu$ erscheint auch einige Verse weiter in 6,19 im Hinblick auf die „Glieder“ (ergänzt durch die Objektsartergän-

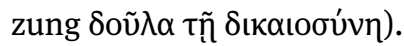

An diesem Beispiel zeigt sich mustergültig, wie ein von Paulus „begehrtes“ Ereignis mit Vergangenheit, Gegenwart und alternativer (d.h. passiv prognostizierter) Zukunft zusammenhängt. Denn mit demselben Verb ist im Ind. Aor. in einer Komparativperiode in V. 19 auf das frühere Verhalten der Angesprochenen Bezug genommen: Der Dienst der Gerechtigkeit zur Heiligung soll nun (vṽv) erfolgen, „wie ihr eure Glieder in den Dienst der Unreinheit und Ungesetzlich-

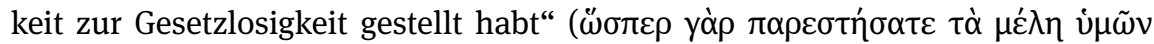

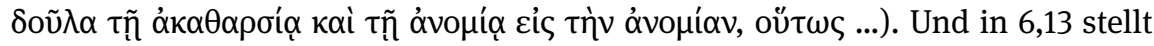
die Aufforderung zur Verfügbarkeit gegenüber Gott das positive Gegenstück zu einem negierten Durativ-Imperativ dar, in welchem das Zurverfügungstellen der Glieder (das Objekt ist hier dasselbe wie in 6,19) für die Sünde als Werkzeuge der

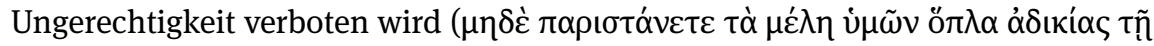
$\dot{\alpha} \mu \alpha \rho \tau i ́ \alpha)$.

In welcher Beziehung stehen die Sachverhalte zueinander, die hier durch Formen desselben Verbs ausgedrückt werden? Es wird in der Diskussion der bedingten Ereignisse (Abschnitt 5.2.1.2) noch zu zeigen sein, wie Paulus hier mit Hilfe von Konditionalsätzen ein Netz an möglichen Handlungssträngen aufspannt. Es stellt sich dabei heraus, dass diese Passage in Röm 6 sich dadurch auszeichnet, dass sie die Angesprochenen dazu ermutigt, die Plots verschiedener Protonarrative miteinander abzugleichen, um so zu einer möglichst guten „Fortschreibung“ ihres Lebens als Christen zu gelangen. ${ }^{267}$ An dieser Stelle soll nun schon einmal vorbereitend zur dortigen Diskussion auf die Funktion des Imperativs innerhalb dieses Ereignisgefüges eingegangen werden.

266 Im Hinblick auf den Imperativ scheint diese Bezeichnung überwiegend angezeigt. Siehe GGNT 212cd.

267 Vgl. Ryan, „Narrative,“ 345 zur Idee „that living one’s life and reflecting upon it is like writing one's life story.“ 
Der Rückgriff in die Vergangenheit der Römer in 6,13 durch den Aorist sagt zunächst nichts darüber aus, wie lange dieses Verhalten an(ge)dauert (hat), ${ }^{268} \mathrm{da}$ nicht die Aktivität des Sündigens selbst als temporal beschränkt dargestellt wird, sondern lediglich auf den zum - so oder so in der Vergangenheit zum Abschluss gekommenen - Übergang zum sündigen Verhalten selbst Bezug genommen wird. Paulus wünscht sich sicherlich einen Bruch, der bereits mit der Taufe stattfand denn in dieser verortet er den Herrschaftswechsel (vgl. 6,4.6). Zugleich trägt seine Formulierung des Textes der Tatsache Rechnung, dass die bisherige „Glaubensgeschichte“ der einzelnen Gläubigen nicht in dem Maß vom Protonarrativ ihrer unveränderten Lebensweise als Unbekehrte abweicht (also von der „passiven Prognose“ zum Zeitpunkt vor der Bekehrung), wie dies in Paulus' Augen angebracht beziehungsweise möglich wäre. Das motivierende Potenzial des Texts steckt daher nicht zuletzt in dem Umstand, dass das jetzt (vṽv) zu Tuende ${ }^{269}$ einerseits in aller Deutlichkeit betont wird, die Aussagen über die Vergangenheit und Gegenwart der Angesprochenen jedoch eine Offenheit aufweisen, die diesen ermöglicht, eine kritische Selbstreflexion im Abgleich der Protonarrative vorzunehmen.

Entsprechend werden manche Römer den aoristischen Dienst an der Gesetzlosigkeit in 6,19 schuldbewusst bis an die Gegenwart heran fortwirkend in ihrem Leben erkannt haben. Für sie wird in $\mu \eta \delta \varepsilon \dot{~} \pi \alpha \rho \iota \tau \tau \alpha ́ v \varepsilon \tau \varepsilon$ (...) in 6,13 eine Aufforderung ausgesprochen gewesen sein, mit ihrem Handeln aufzuhören. ${ }^{270}$ Dieses Verständnis eines verneinten durativen Imperativs als STOP-DOING-X-Anweisung (anstelle einer „general prohibition against a particular behavior“271) erfordert (in der exegetischen Literatur so nahezu nie berücksichtigt) eine sehr spezifische Konstellation im Text, die hier allerdings tatsächlich gegeben zu sein scheint. ${ }^{272}$

\section{GGNT 199b.}

269 Es ist nicht unproblematisch (wie etwa Wolter, Brief I, 389), davon zu sprechen, dass der Aorist ein ,ingressives“ Element beisteure. Vielmehr ist es wohl so, dass das Aktionsartpotenzial telisch verstanden werden kann (BDAG 5697,1a), sodass sich die Aufforderung gut mit einem singulären transformativen Akt verbinden lässt. (Vom Beginn eines Handelns kann deswegen aber eigentlich nicht die Rede sein.) Römische Christen, die Röm 6,19 ausschließlich auf ihre vorchristliche Vergangenheit bezogen haben, dürften andererseits wiederum hier in V. 13 wohl eher an ein weniger punktuelles ,Präsentieren` (vgl. BDAG 5697,1bo) gedacht haben. Für sie hätte das Aufgeforderte in der Fortsetzung einer bereits aufgenommenen Aktivität bestanden.

270 Richtig Cranfield, Epistle II, 317.

271 Aubrey, „Prohibitions,“ 504.

272 Die Darstellung bei GGNT 212e ist im Lichte der Forschung von Aubrey, „Prohibitions“ zu präzisieren: Notwendig für eine „Aufforderung, mit der (bereits begonnenen) ,Handlung ‘ ... aufzuhören“ ist (Aubrey, „Prohibitions, “ 534) ,imperfective aspect, imperative mood, nuclear-scope negation, a specific, referential event.“ Am problematischsten ist wohl der letzte Punkt, welcher 
Wer wiederum die in 6,19 mit indikativischem $\pi \alpha \rho \varepsilon \sigma \tau \eta ́ \sigma \alpha \tau \varepsilon$ bezeichnete Handlung als weit in der Vergangenheit - bei der Bekehrung - zum Ende gekommen ansieht, wird denselben Imperativ in V. 13 als Aufforderung, grundsätzlich nicht nach einem solchen Maßstab zu handeln, verstanden haben. ${ }^{273}$

Diese grundsätzliche Offenheit eines verneinten durativen Imperativs ist zumindest bei Verben mit einem Aktionsartpotenzial des Typs „activity“ möglich. ${ }^{274}$ Die Aufforderung in Röm 6,13 ist also wohl tatsächlich und bewusst polyvalent $^{275}$ und erlaubt auf Rezipientenseite ein unterschiedliches Verständnis, abhängig davon, wie sich der einzelne Leser im Geschehenszusammenhang verortet, der in Kapitel 6 des Briefes zur Sprache kommt. Es zeigt sich hier erneut, dass der Verzicht auf eine rein rezeptionsorientierte Auffassung mentaler Narrative heuristisch wertvoll ist (vgl. Kapitel 10, Abschnitt 4 gegen die Adaption von Ryans Konzeption durch Finnern und Rüggemeier): Die individuellen Protonarrative der Rezipienten sind Teil der komplexen Kommunikationsintention des Autors, welcher sowohl das Ereignisnetz bereitstellt, innerhalb dessen die

auch mit der Aktionsart zusammenhängt. Vgl. zu Aktivitäten die Diskussion zu Eph 4,26 ( $\mu$ ท̀

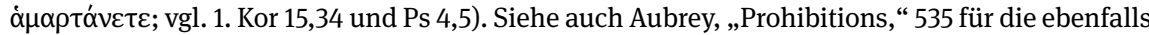
spezifischen notwendigen Bedingungen für die DO-NOT-START-X-Bedeutung.

273 Wilckens, Brief II, 20 geht von diesem Verständnis aus - kombiniert es jedoch mit einer Überinterpretation des aoristischen Aspekts: „Sie sollen ihre Glieder nicht als Waffen der Ungerechtigkeit der Sünde, sondern sich selbst Gott zur Verfügung stellen. Der Aorist ... in V13b aktualisiert, was das Präsens ... in V13a generell sagt. In der ganzen Zeit christlichen Lebens im sterblichen Leib soll es grundsätzlich keinerlei Dienstleistung für die Sünde in Taten der Ungerechtigkeit geben, sondern in jedem Augenblick geht es darum, Gott in Taten der Gerechtigkeit zu dienen.“

274 Vgl. Aubrey, „Prohibitions,“506-507.

275 Thesen zu intentionaler Ambiguität sind grundsätzlich sehr problematisch. In den meisten Kontexten widersprechen sie den Konversationsannahmen (GGNT 314). Für die gezielt eingesetzte Mehrdeutigkeit (als Stilfigur der „Amphibolie“) siehe GGNT 296g. Hier läge jedoch eher ein Fall von bewusster „Mehrdeutbarkeit“ vor: Röm 6,13 weist mehrere mögliche Lesarten auf, ist aber zugleich für verschiedene Äußerungskontexte zugleich verfasst. In jedem der beiden Äußerungskontexte kommt es zu einer Disambiguierung, sodass jeweils nur eine mögliche Äußerungsbedeutung übrig bleibt. Vgl. Option (b) bei Löbner, Semantik, 65. Auch wenn man mit solchen Thesen sparsam umgehen sollte, erscheint die hier vorgeschlagene Klassifizierung aufgrund weiterer Indizien im Kapitel möglich. Alternativ stellt sich die Frage, was die hier postulierten „Vorzeigechristen“ wohl zum durativen Imperativ in 6,13 gesagt hätten. Geht man von der Stoßrichtung „Hört auf zu sündigen!“ als einzig möglicher Leseweise aus, müssten sie eine solche Aufforderung doch als recht verwirrend empfunden haben - einerseits als Affront, andererseits aber auch als reichlich unerwartet angesichts dessen, was Paulus bisher selbst (z. B. in V. 10) gesagt hatte. Eher noch wäre bei monosemer Auslegung zu erwägen, dass das generelle Verbot die einzige intendierte Lesart war. Allerdings ist der explizite Wechsel des Aspekts innerhalb von V. 13 dann schwer verständlich. 
Konstruktion der eigenen „Geschichte“ zu erfolgen hat, als auch die polyvalenten narrativen Weichenstellungen anregt.

\subsubsection{Der voluntative Konjunktiv in Röm 13,11-14}

Neben den Imperativ-Formen sind wie eingangs erwähnt auch die voluntativen konjunktivischen Verben im Hauptsatz $\mathrm{zu}$ beachten. Sie können einerseits (meist in der 2. Person) mit $\mu$ '́ verneint in (a) prohibitiver Funktion als Ersatz des nicht gebräuchlichen verneinten Aorist Imperativs auftreten. ${ }^{276} \mathrm{Im}$ Römerbrief liegt dieser Gebrauch nur in Röm 10,6 vor: „Sprich nicht in Deinem Herzen ...“ ( $\mu$ tion der Aufforderung für die 1. Person Singular/Plural im (b) adhortativen Gebrauch. ${ }^{277}$

Im Römerbrief sind nur wenige Verbformen dieser Kategorie zuzuordnen. ${ }^{278}$ Ein Beispiel soll wiederum genügen, um zu verdeutlichen, wie das zukünftige Ereignis, welches angestrebt wird, in einen größeren Ereigniszusammenhang eingefügt ist. Die besprochene Passage Röm 13,11-14 ist insbesondere auch deswegen äußerst interessant, weil sich hier tatsächlich eine „narrative Substruktur“ im Sinn von Hays nahezulegen scheint: Die Verse 12-14 werden dadurch zusammengehalten, dass sie auf einen bestimmten Abschnitt eines zugrunde gelegten Narrativs (V. 11a) fokussieren. Auch die Imperative in V. 14 und eben die adhortativen Konjunktive in V. 12 und 13 erhalten ihren Zusammenhang durch diesen narrativen Rahmen.

\footnotetext{
${ }^{11}$ Und das tut, weil ihr die Zeit erkannt habt, dass die Stunde da ist, aufzustehen vom Schlaf, denn unser Heil ist jetzt näher als zu der Zeit, da wir gläubig wurden.

${ }^{12}$ Die Nacht ist vorgerückt, der Tag ist nahe herbeigekommen. So lasst uns ablegen

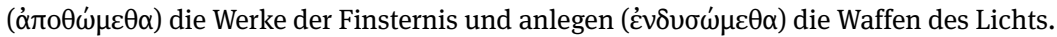

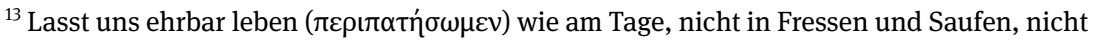
in Unzucht und Ausschweifung, nicht in Hader und Neid;

${ }^{14}$ sondern zieht an den Herrn Jesus Christus und sorgt für den Leib nicht so, dass ihr den Begierden verfallt. ${ }^{279}$
}

In Röm 13,12b fordert Paulus zum „Ablegen der Werke der Finsternis“

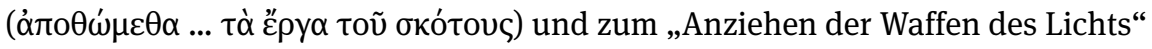

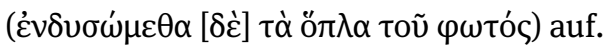

276 GGNT 210e.

277 GGNT 210c.

278 Und zwar in Röm 13,12 (2x), 13,13, 14,13, 14,19.

279 Luther 2017. 
Interessanterweise wird diese metaphorische Aufforderung $\mathrm{zu}$ einer Transformation des Lebenswandels durch oũv eingeleitet, wodurch eine konsekutive Konnexion des Typs Grund-AUFFORDERUNG markiert wird, ${ }^{280}$ wobei mit Äußerungsbezug die an „uns“ ergehende Aufforderung als Folge eines Geschehens dargestellt wird. Dieser Grund besteht nun in einer kleinen Miniaturerzählung, die in Röm 13,11a beginnt: Das Römer sollen das Liebesgebot befolgen, ${ }^{281}$ weil sie

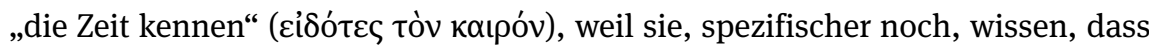

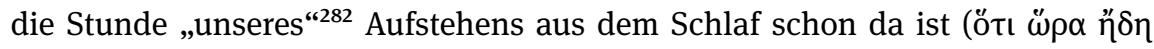

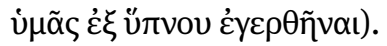

Zunächst wird dieses Bild in V. 11b auf die nahende Errettung bezogen. Nach dieser Übertragung, fährt V. 12a asyndetisch fort und greift dadurch zurück auf das aufgerufene Bild des Tagesanbruchs: „Die Nacht ist vorgerückt und der Tag

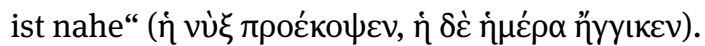

Die auf dieser temporalen Verortung fußende doppelte Selbstaufforderung in V. 12a greift dann den Licht-Dunkel-Dualismus auf: Es ist Zeit, in den Tag zu starten (V. 11a und 12a), und die „Ausstattung“ soll dem entsprechen. Damit wird die Erzählung aufgegriffen, wenn auch nicht fortgeführt, da die Narration auf der Stelle tritt. Wie können dann die zwei Imperative in V. 14, die das erwünschte Verhalten spezifizieren, integriert werden?

Festgehalten werden muss zunächst, dass auch sie nicht völlig losgelöst von der im Hintergrund stehenden Erzählung zu verstehen sind. Das zeigt eindeutig die in V. 13a eingeschobene Formulierung auf, wo Paulus die Situation auffälligerweise nochmals explizit durch einen weiteren adhortativen Konjunktiv auf-

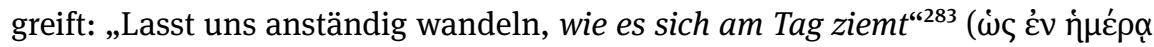

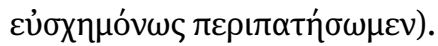

Vor diesem Hintergrund betrachtet, scheint es doch sehr bemerkens-

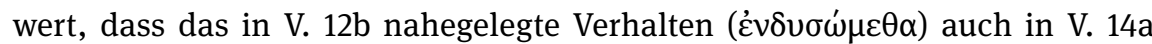

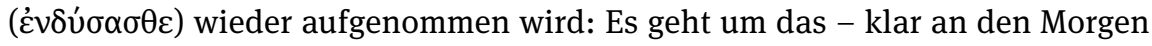
erinnernde - „Anziehen.“284 Auch der verneinte Imperativ in V. 14b lässt sich schließlich vor diesem Hintergrund angemessen integrieren, auch wenn die

280 Vgl. GGNT 333a und 334a und oben, Kapitel 5, Abschnitt 3.4 zur hier vorgenommenen Abgrenzung von Konsekutivität gegenüber Kausalität.

281 Vgl. NSS 942-943 zur verblosen Anknüpfung an 13,8-10.

282 Zur Textkritik der Stelle siehe Caragounis, Development, 521.

283 Vgl. NSS 943.

284 Diese Aussage gilt natürlich nicht bei bestimmten ausgefallenen kriegerischen Taktiken. Pausanias, Periegesis 10.1.11 hält beispielsweise folgende Episode fest, welche faszinierende Berührungspunkte mit dieser Passage aufweist: Eine Gruppe Phoker richtet ein Blutbad unter Thessaliern

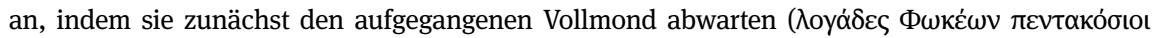

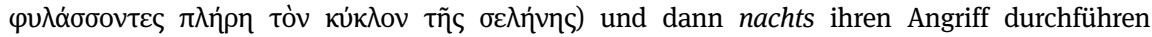


Mahnung, „nicht Vorsorge für das Fleisch zu treiben, um Begierden zu erregen“

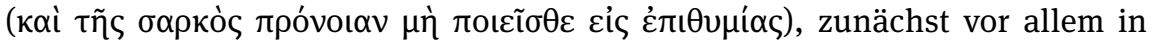
der Lebenswirklichkeit der Christen verankert $\mathrm{zu}$ sein scheint. Am ehesten noch erinnert die Rede vom „wach werden“ der Begierden in manchen Übersetzungen (z. B. Elberfelder) an die Situation des morgendlichen Starts in den Tag - wobei dann allerdings gerade die sorgfältig um die Erzählfigur herum konstruierte Konstellation ins Gegenteil verkehrt wird. Denn das konkret gemeinte Verhalten, das abgelehnt wird und in den modalen Dativen in V. 13b

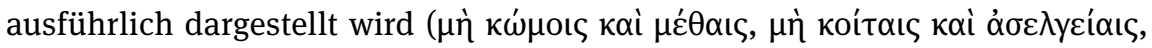
$\mu \eta ่$ werden: Es handelt sich dabei um Handlungen, die der Schändlichkeit wegen eigentlich nur im „Dunkel“ ausgeführt werden. Es sind eben, wie V. 12b sagt,

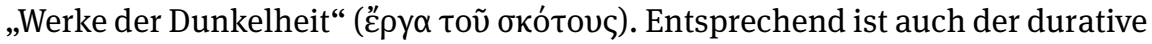
Imperativ ${ }^{285} \pi$ тогा $\sigma \theta \varepsilon$ in V. 14b zu verstehen: So wie in V. 12b dazu aufgefordert wurde, die entsprechenden Werke „abzulegen,“286 wird hier darauf insistiert: „Beschäftigt euch nicht länger damit, wie ihr die Begierden eurer eigenen Natur zufrieden stellen könnt.“287

Unabhängig von der traditionsgeschichtlichen Herleitung von V. 11-12 aus einem Tauflied ${ }^{288}$ und dem eschatologischen Sachbezug der Metapher des „Aufstehens“289 ist folglich bei näherer Betrachtung doch sehr auffällig, dass Paulus die „erzählte Welt“ in den Aufforderungen nicht verlässt, sondern die Situation einer den Morgen erwartenden Erzählfigur weiter voraussetzt.

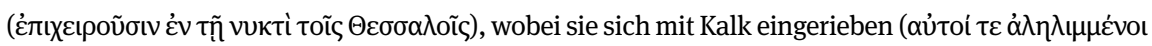

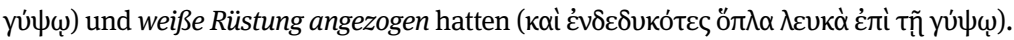

285 Siehe den vorangehenden Abschnitt 4.2.2 zu den Bedingungen des STOP-DOING-X-Musters. 286 Für ein unterlassenes Ablegen der Waffen bei Nacht mit demselben Verb siehe Plutarch,

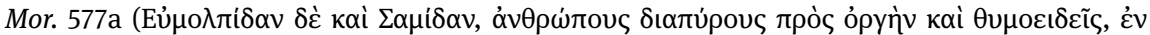

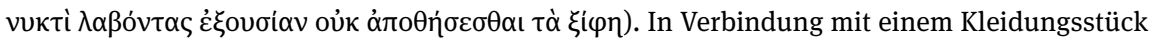

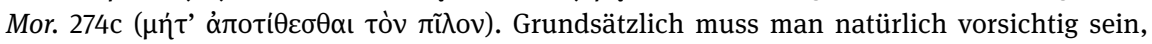
hier keine anachronistischen Vorstellungen über das morgendliche Ablegen von „Nachtwäsche“ einzutragen. Siehe etwa Hld 5,3, wo die abendliche Situation des Ausziehens des Un-

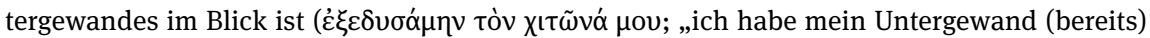
ausgezogen“). Immerhin setzt Lk 9,3 (anders als Mk 6,9, wo wohl Schutz vor Kälte im Blick ist)

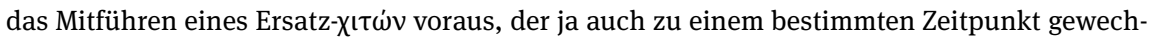
selt werden musste. Siehe für Nachtwäsche und die Morgenroutine aber auch Dickey, Stories, 23-25.42.

287 NGÜ.

288 Wilckens, Brief III, 75.

289 Wilckens, Brief III, 76. 


\subsection{Selbstständige Begehrsätze: Desiderativsätze}

\subsubsection{Kupitiver Optativ}

Als zweite große Klasse innerhalb der Begehrsätze treten Wunschsätze (Desiderativsätze) neben die Kategorie der Aufforderungen. ${ }^{290}$ Den „als erfüllbar hingestellten Wunsch“291 drückt dabei der (sogenannte kupitive) Optativ aus. Im

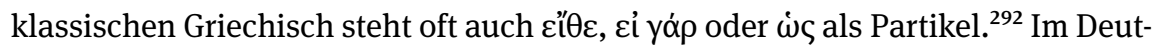
schen erscheint in analogen Situationen der Konjunktiv I in selbstständigen Verbzweitsätzen (mit dem Verb in der 3. Person; z. B. „Er lebe hoch!“‘), ${ }^{293}$ wobei zwischen der Funktion des Wunsches, der Bitte und der Aufforderung nicht klar unterschieden werden kann. ${ }^{294}$

Primär erscheint diese Verwendungsweise im Römerbrief in der Abweisungs-

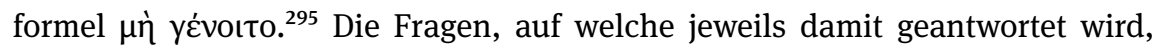
weisen dabei recht große Unterschiede auf. So wird in Röm 7,7 etwa eine Klassifizierungsfrage aufgeworfen: „Ist das Gesetz Sünde?“ (ó vó $\mu$ o $\dot{\alpha} \mu \alpha \rho \tau i ́ \alpha)$. An anderen Stellen sind jedoch keine Konstellationen oder Definitionen im Blick, sondern tatsächlich Ereignisse. So wird in 6,2 auf das in 6,1 durch die Frage ${ }^{296}$ aufgeworfene Protonarrativ eines Verharrens in der Sünde mit dem Zweck des

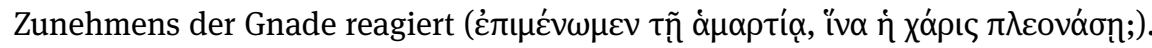
In 6,15 wird ebenfalls ein zukünftiger Handlungszusammenhang ausgeschlossen, der sich aus dem gegenwärtigen Zustand ergeben könnte: das Sündigen aufgrund der Tatsache, dass man sich nicht unter Gesetz, sondern unter Gnade befindet

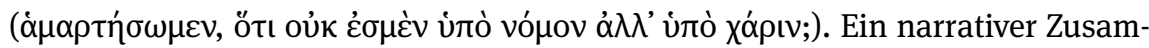
menhang findet sich auch in 7,13 und 11,1.11, wobei hier eine andere Perspektive

$290 \mathrm{Zu}$ den formalen Gestaltungsmöglichkeiten für Wünsche in selbstständigen Begehrsätzen siehe GGNT 268b. Die Terminologie wurde hier etwas abgeändert (vgl. Duden 1403), da die Rede von einem „Wunsch“ auch schlicht eine innerliche Einstellung implizieren kann und auf diesem Wege leicht auch auf Äußerungen ausgeweitet wird, in denen ein abhängiger Behauptungssatz auf Verben des Sehnens etc. folgt. Vgl. oben, Abschnitt 3.4 zu den „erhofften“ Ereignissen.

291 GGNT 211c.

292 Vgl. GGNT 211c: „typischerweise“ (wohl vor dem Hintergrund von BR: „meist“). CGCG 38.38 formulieren nun: „sometimes,“ ohne jedoch explizit auf die Hintergründe einzugehen.

293 Duden 780. Die 1. Person Plural ist in Erststellung ebenfalls möglich, wird dann aber als sprechereinschließende „höfliche Form der direkten Aufforderung“ verstanden (z. B. „Seien wir doch vernünftig.“).

294 Duden 779.

295 Röm 3,4.6.31, 6,2.15, 7,7.13, 9,14, 11,1.11. Zur Einordnung unter den kupitiven Optativ vgl. GGNT 211c.

$296 \mathrm{Zu}$ Fragen und Protonarrativen vgl. oben Abschnitt 2. 
eingenommen wird: Bei diesen Stellen geht es um die Interpretation der bisher geschehenen Ereignisse. ${ }^{297}$

Semantisch garantiert die Formulierung - anders als es oủ $\mu \eta \dot{~ \gamma \varepsilon ́ v \eta \tau \alpha ı ~ t a ̈ t e ~-~}$ gerade nicht den (behaupteten) Erfolg des Wunsches. ${ }^{298}$ Auch wenn die pragmatische Funktion im Kontext der rhetorischen Fragen durchaus einem „Auf keinen Fall!“ nahekommt, ist für die Rekonstruktion von Protonarrativen also durchaus bedeutsam, dass hier mögliche zukünftige Handlungsabläufe aufgerufen und von Paulus als inakzeptabel qualifiziert, damit aber nicht automatisch vollständig ausgeschlossen sind. ${ }^{299}$

Der kupitive Optativ erscheint auch an zwei weiteren Stellen im Römerbrief, außerhalb dieses spezifischen Gebrauchs, in auf die Adressaten bezogenen Wünschen: Der Gott „der Geduld und des Trostes,“ so formuliert Paulus in Röm 15,3 als Wunsch, möge den Römern geben ( $\delta \omega \hat{\eta}$ ưñ̃v), Jesus Christus gemäß ,unterei-

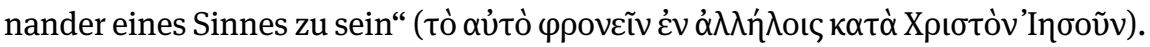

Auch in 15,13 ist wieder Gott derjenige, dessen Handeln das Erwünschte ist und wieder wird er durch ein Genitivattribut näher beschrieben („Der Gott der

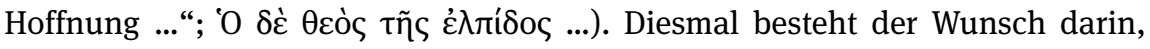
dieser Gott möge die Römer „mit aller Freude und jedem Frieden erfüllen durch

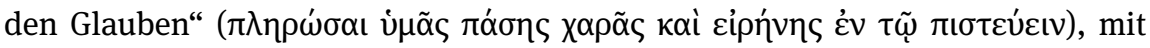
dem Ziel, dass sie überreich in der Hoffnung würden durch die Kraft des Heiligen

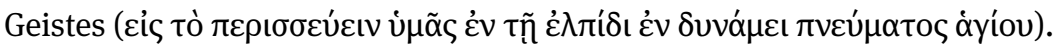

Das Attribut qualifiziert Gott in beiden Fällen als die Quelle von Geduld und Trost beziehungsweise Hoffnung. Es erfüllt damit eine ähnliche Funktion wie Aussagen über Gottes Vermögen in Bezug auf futurische Ankündigungen an anderer Stelle. ${ }^{300}$

Neben solchen allgemeinen Segenswünschen tritt der kupitive Optativ in 1. Thess 3,11 auch zum Ausdruck eines recht konkreten Wunsches in Erscheinung: Während in 3,10 noch ein in die Frage 3,9-10 eingekleideter Bericht darüber vorliegt, dass Paulus und seine Mitarbeiter (vgl. 1,1) Tag und Nacht Gott darum bitten, er möge es ihnen ermöglichen, die Thessalonicher wiederzusehen

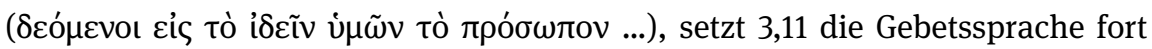

297 Es handelt sich also um eine besonders schwache Variante des in Kapitel 12, Abschnitt 6 diskutierten Fall eines „graduellen“ Diserzählens vergangener Ereignisse, wobei es primär um die Bewertung von Geschehnissen geht (siehe dazu auch Kapitel 8, Abschnitt 4).

298 Vgl. etwa Lk 20,16.

299 Vgl. Wallace, Grammar, 481-482.

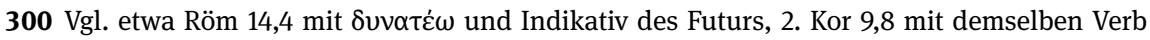
und einer Finalangabe. 
und formuliert den Wunsch „unser Gott und Vater selbst“ und „unser Herr Jesus“

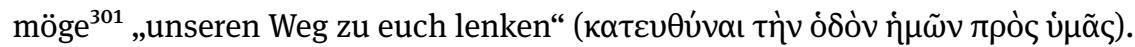

Ein konkretes Verhalten ${ }^{302}$ ist zweifellos auch in dem Wunsch ins Auge genommen, den Paulus in Phlm 20 für sich selbst formuliert - dass er nämlich

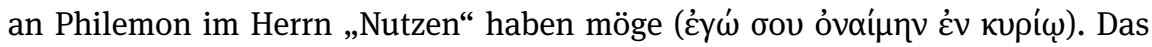
Verb óvíva $\mu \alpha$ เ bedeutet konkret ,to be the recipient of help or favor. ${ }^{303}$ Nebenbei bemerkt sei, dass es nur schwer vorstellbar ist, dass hierbei keine Anspielung auf

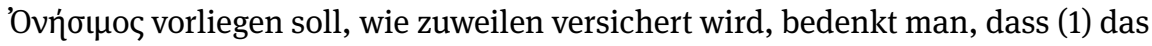
Verb im gesamten biblischen Kanon kein zweites Mal belegt ist, (2) das Wortspiel in V. 11 einen ganz ähnlichen Gedanken voraussetzt und (3) die Lautkombination /oni/ (ganz zu schweigen von /one/) als Beginn des Stamms eines Wortes sowieso äußerst selten ist. ${ }^{304}$

\subsubsection{Augmentindikativ ohne öv}

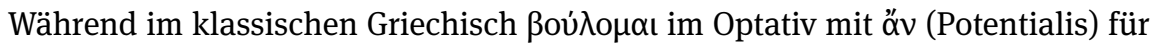
erfüllbare Wünsche verwendet werden konnte und Augmenttempus mit őv (Irre-

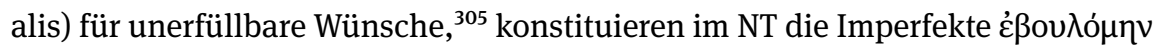

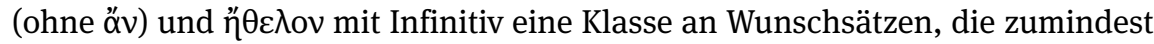
teilweise als erfüllbar angesehen werden können (siehe aber oben, Kapitel 12, Abschnitt 8.4 für unerfüllbare Wünsche).

Bei Paulus wird diese Verwendungsweise für Phlm 13 diskutiert, wobei hier wohl eher eine Aussage über ein in der Vergangenheit anzusiedelndes Verlangen gemacht wird (siehe jedoch die Diskussion der Stelle unten, Abschnitt 5.3.2.4). Auch die Imperfekt-Form nủxó $\mu \eta v$ in Röm 9,3 gehört wohl in diese Kategorie: Es handelt sich hierbei um einen Wunsch, den Paulus zumindest äußern könnte. ${ }^{306}$

301 Zum Singular vgl. Wallace, Grammar, 482.

302 Recht allgemein gehaltene Wünsche, die jedoch in sehr spezifischem Verhalten begründet werden, finden sich in 1 . Tim 1,16.18 und 2. Tim 4,16.

303 LN 35.4 .

304 Bedenkt man sämtliche den Lautwandel berücksichtigenden Kombinationen, so bleibt im NT neben Mk 9,42/Mt 18,6 (óvıkós) und Kol 4,9 und 2. Tim 1,16; 4,19 - dort die Namen 'Ovńбuos

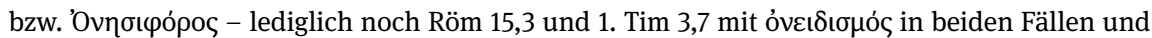

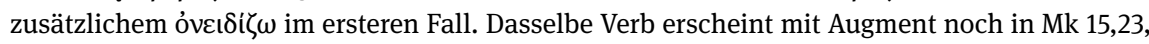

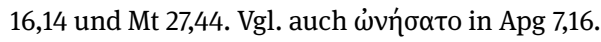

305 GGNT 268b.

306 Vgl. Caragounis, Development, 163 mit Verweis auf das neugriechische Äquivalent $\theta \alpha$

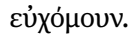




\subsection{Abhängige Begehrsätze und Infinitiv-Konstruktionen}

\subsubsection{Hinführung}

Der oben (Abschnitt 4.2.1) genannte Sonderfall eines ,imperativischen“ Infinitivs ist auf das Ausfallen einer übergeordneten Konstruktion mit einem Verb des Begehrens zurückzuführen. ${ }^{307}$ Auch die hier als selbstständig klassifizierten Desiderativsätze (Abschnitt 4.3) weisen zumindest eine partikelhafte finite Verbform in der Konstruktion auf. Dies führt uns nun zur überblicksartigen Betrachtung der abhängigen Begehrsätze als potenzielle Quellen für Erzählfragmente in den Paulusbriefen. ${ }^{308}$

Denn auch wenn im NT der klassische Gebrauch ${ }^{309}$ des Infinitivs als Akkusativobjekt nach Verben des Begehrens und Wünschens noch üblich ist, ${ }^{310}$ führt die nachklassische Ausbreitung von ǐv $\alpha$ als Ersatz für andere Konstruktionen ${ }^{311} \mathrm{zu}$ einer nahezu vollständigen Austauschbarkeit mit dem Infinitiv im Neuen Testament ${ }^{312}$ und somit auch zu vielen Fällen, in welchen ein Objektsatz mit iv $\alpha$ einen abhängigen Begehrsatz einleitet. ${ }^{313}$ Diese beiden grundsätzlichen Konstruktionsmöglichkeiten treten bei den verschiedenen Verben in sehr unterschiedlicher Verteilung auf. ${ }^{314}$

Beim im Folgenden gebotenen Durchgang durch die relevanten Verben des „Begehrens und Wünschens“315 muss natürlich einerseits berücksichtigt werden, dass die semantische Abgrenzung zu Verben des Hoffens, Sehnens etc. (siehe oben, Abschnitt 3.4) nicht immer eindeutig ist. Teilweise ist die Entscheidung zwischen unterschiedlich relevanten Bedeutungen polysemer Ausdrücke auch

307 GGNT 218d.

308 Für einen Überblick, siehe GGNT 272.

309 CGCG 51.8.

310 GGNT 218a.

311 Vgl. Caragounis, Development, 218-226.

312 Siehe GGNT 272a.

313 Siehe GGNT 272b für eine Übersicht über die rekonstruierten selbstständigen Begehrsätze, die im Hintergrund stehen. Sehr selten begegnet im NT auch die Ersatzkonstruktion mit ö $\pi \omega \varsigma$, die hier nicht weiter diskutiert werden soll.

314 Eine gute Übersicht für die jeweiligen Konstruktionsmöglichkeiten mit Begehrverben findet sich bei BDR 392. Auch die hier exemplarische aber für die besprochenen Verben ausführlichere Diskussion gibt einen Eindruck von der unterschiedlichen Verteilung der syntaktischen Auflösungen.

315 Der folgende Abschnitt geht von der Liste in GGNT 218a aus. Da diese nicht vollständig ist, wurden zudem in einigen Fällen Suchen mit den entsprechenden Louw-Nida-Kategorien durch-

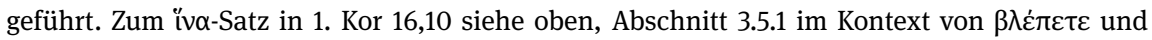
Befürchtungssätzen. 
schwierig. So könnte $\theta \varepsilon \dot{\lambda} \omega$ in Kol 1,27 sowohl einen Begehren zum Ausdruck bringen als auch ein „Planen.“316

Grundsätzlich lassen sich die hier diskutierten abhängigen Konstruktionen als Umformulierungen selbstständiger Begehrsätze verstehen, ${ }^{317}$ wobei die Sprechhandlung durch ein übergeordnetes Verb im Text selbst spezifiziert wird. Dabei ist natürlich zu beachten, dass die pragmatische Funktion von der expliziten Benennung abweichen kann: Eine als Hoffnung qualifizierte Aussage kann in einer konkreten Gesprächssituation durchaus als Appell fungieren. ${ }^{318}$ Andersherum kann eine der Form nach als Aufforderung erscheinende (d.h. etwa mit einem Verb wie „befehlen“ konstruierte) Aussage auch lediglich eine Hoffnung ausdrücken, ohne durch den Sprechakt auf die Situation einwirken zu wollen. ${ }^{319}$

Demgegenüber ist zu beachten, dass sich die folgende Übersicht primär an einer lexikalisch-semantischen Klassifikation orientiert: ${ }^{320}$ Die Verben des „Wün-

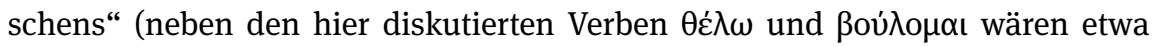

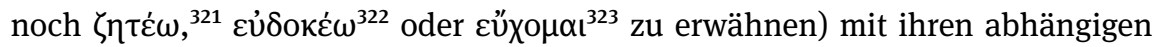
Konstruktionen würden umformuliert in selbstständige Begehrsätze wohl in der

316 Vgl. LN 25.1 (,desire`) und 30.58 (,purpose‘).

317 Siehe GGNT 272b für eine beispielhafte Gegenüberstellung von selbstständigen und abgeleiteten abhängigen Begehrsätzen.

318 Siehe oben, Abschnitt 4.1 in der Hinführung zu Begehrsätzen.

319 Vgl. etwa im Deutschen ein bloß zu sich oder einer dritten Person gesagtes/gezischtes: „Er soll endlich still sein!“ oder sogar „Halte doch endlich die Klappe!“

320 Danove, New Testament Verbs konnte für diese Arbeit leider nicht mehr berücksichtigt werden, wäre hier generell aber zweifelsohne ein sehr relevanter Gesprächspartner.

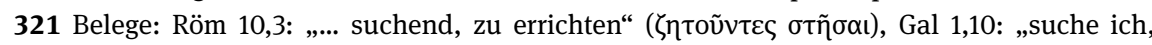

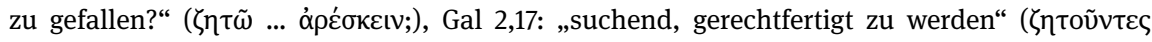
$\delta เ \kappa \alpha \iota \omega \theta \tilde{\eta} v \alpha \mathrm{l})$. Die Definition ,to desire to have or experience something, with the probable implication of making an attempt to realize one's desire (LN 25.9) ist manchmal nicht deutlich von anderen Bedeutungen des Verbs abzugrenzen. Dies gilt insbesondere auch für die beiden Belege

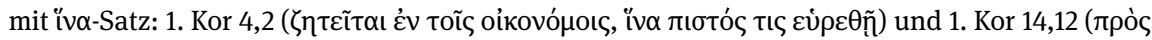

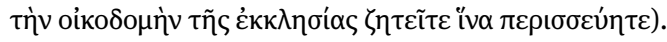

322 Präsentisches $\varepsilon \dot{\delta} \delta$ ok $\varepsilon \dot{\omega}$ erscheint in 2. Kor 5,8 und 2. Kor 12,10. (1. Thess 2,8 ist wohl als Imperfekt zu lesen). In 2. Kor 12,10 ist das Verb jedoch intransitiv gebraucht (mit év). Siehe zum Kontext unten, Abschnitt 5.2.2.3. In 2. Kor 5,8 wird durch $\mu \tilde{\alpha} \lambda$ Aov mit Infinitiven ein Vorzug markiert: „,... und wir möchten lieber ausheimisch vom Körper und einheimisch beim Herrn

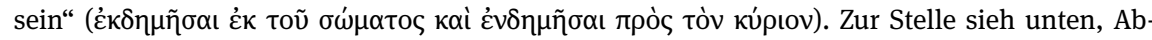
schnitt 4.4.2.1.

323 Zur Unterscheidung der das Wünschen betreffenden Bedeutung und der spezifischer auf das Gebet bezogenen vgl. LN 33.178 und 25.6. Einziger Beleg eines Wunsches bei Paulus könnte

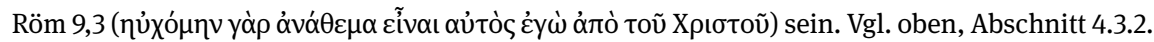

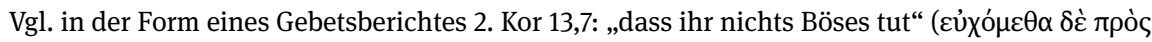

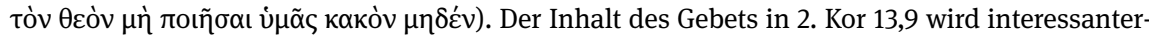


Regel schlicht im Modus des Imperativs stehen. ${ }^{324}$ Ein (1) Verb des Wünschens kann also durchaus eine Sprechhandlung einführen, die vom Kontext her als eine autoritative Instruktion zu verstehen ist, wie dies bei der Großkategorie der (2) „Verben des Aufforderns“ standardmäßig der Fall ist. Dieser Bereich des Aufforderns lässt sich wiederum in (2a) „Verben des Bittens“ und (2b) „Verben des Befehlens “ unterteilen, ${ }^{325}$ wobei hier die abhängige Konstruktion gegenüber dem erwartbaren Imperativ (bzw. Konjunktiv) in der selbstständigen Konstruktion eine Differenzierung in der Beziehung zwischen Sprecher und Angesprochenem im Hinblick auf das Aufgeforderte vornehmen kann. ${ }^{326}$

\subsubsection{Verben des Wünschens}

\subsubsection{1 ,Desire, want, wish': $\theta$ ź̀}

Durchsucht man das corpus Paulinum nach entsprechenden Verben des Wünschens, ergibt sich folgendes Bild: Beim häufigsten Verb des Wollens - $\theta \dot{\varepsilon} \lambda \omega-$ tritt der Inhalt in der überwiegenden Anzahl der Fälle im Infinitiv auf. Die Konstruktion mit iv $\alpha$ findet sich lediglich in 1 . Kor 14,5, interessanterweise par-

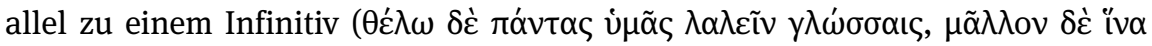

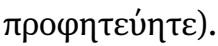

Wichtig ist für die Auswertung der paulinischen Texte auf Protonarrative hin, dass durchaus nicht immer ein bestimmtes Ereignis in der Zukunft ins Auge gefasst wird. Vielmehr kann durch die Verneinung des Infinitivs natürlich auch ein Ereignis diserzählt werden. Dass Paulus mit diesem Verb den Willen ausdrückt, dass etwas nicht geschehen möge, ist allerdings ausgesprochen selten. Ansatzweise ist dies in Röm 13,3 zu sehen, wo in dem als Wenn-Proposition fungierenden Fragesat ${ }^{327}$ die Formulierung ,Willst Du Dich nicht fürchten = Wenn

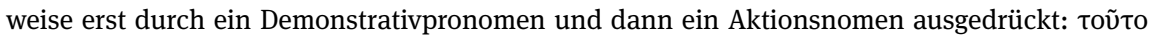

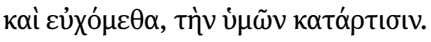

324 Der analoge selbstständige Begehrsatz wäre in den diskutierten Beispielen im NT wohl kaum ein als erfüllbar hingestellter Desiderativsatz im kupitiven Optativ (GGNT 211c). Der Optativ in der 2. Person Sg./Pl. ist im NT (im potenzialen Konditionalsatz) ausschließlich in 1. Petr 3,14 belegt. Vgl. klassisch aber Xenophon, Hell. 4.1.38 bei CGCG 38.38.

325 Vgl. die Auflistung in GGNT 218a. Siehe GGNT 272b für die im Hintergrund stehenden selbstständigen Begehrsätze.

326 Eine gewisse Differenzierung ist auch im Hinblick auf die entsprechenden Hauptsätze möglich. Die Funktion des Deutschen „bitte“ bzw. „please“ übernimmt im Griechischen wohl eine flektierte Verbform von $\delta \varepsilon \dot{\varepsilon} \mu \alpha \mathrm{s}, \dot{\varepsilon} \rho \omega \tau \alpha \dot{\alpha} \omega, \pi \alpha \rho \alpha \kappa \alpha \lambda \dot{\varepsilon} \omega$ etc., an welche der Imperativ asyndetisch

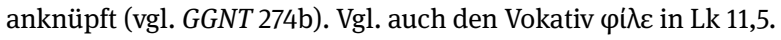

327 Röm 13,3 liefert hierfür einen paulinischen Beleg zu GGNT 331e. 


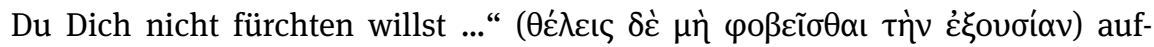
taucht. ${ }^{328}$ Im Weitesten Sinne könnte man Phlm 14 auch hier anführen, denn dort

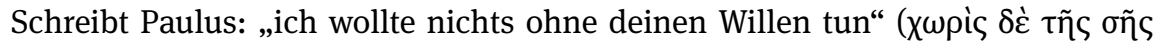

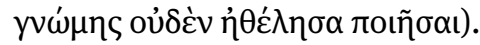

Häufiger ist der Fall, dass der Wille zu einer Handlung selbst verneint wird..$^{329}$ So versichert Paulus etwa in Röm 1,13: „ich will nicht, dass euch unbekannt ist“

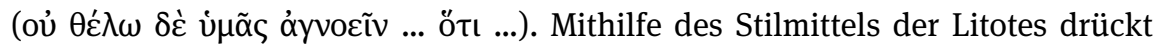
Paulus hier natürlich den positiven Wunsch aus, die Angesprochenen wollten doch eine bestimmte Sache wissen. ${ }^{330}$ Dabei ist nicht an die Möglichkeit einer in ferner Zukunft liegenden Erkenntnis gedacht. Vielmehr fungiert diese Phrase als Einleitungsformel für den darauf folgenden Inhalt. Dieselbe Konstruktion findet sich auch in Röm 11,25 (mit Nomen als Akk0), 1. Kor 10,1 (ötı), 1. Kor 12,1 (intransitiv; Inhalt folgt; V. 2 ruft zuvor nochmal bereits Bekanntes auf), 2. Kor 1,8 (ن் $\dot{\rho} \rho$...) und - im Plural - in 1. Thes 4,13 (gefolgt von $\pi \varepsilon \rho i$ ). ${ }^{331}$

Teilweise wird allerdings durch negiertes $\theta \varepsilon \dot{\lambda} \lambda \omega$ durchaus ein Bezug auf ein konkretes in der potenziellen Zukunft liegendes Ereignis Bezug genommen und als unerwünscht qualifiziert. So etwa in 1. Kor 10,20: „Ich will aber nicht, ${ }^{332}$

328 Im Deutschen kann statt dem Konnektor (v. a. „wenn“) auch schlicht asyndetisch die Inversion stehen: „Wenn Du Dich nicht fürchtest, dann ...“= „Fürchtest Du Dich nicht, dann ...“ Vgl. GGNT 280a. Dieselbe Satzstellung mit finitem Verb in erster Position weist auch die entsprechende Entscheidungsfrage auf: „Fürchtest Du Dich nicht?“ (vgl. Duden 1343). Im Griechischen bietet der Fragesatz somit eine ganz ähnliche syntaktische Lösung für asyndetische Konnexionen des konditionalen Typs.

329 Verneintes $\theta \varepsilon \dot{\lambda} \lambda \omega$ drückt - wie das deutsche „Ich will/möchte nicht, dass ...“ - nicht einfach die Abwesenheit eines positiven Wunsches, sondern das Vorhandensein eines negativen Begehrens aus.

330 GGNT 296h. Vgl. oben, Kapitel 8, Abschnitt 5.2.2 zur narrationsspezifischen Aufgabe des Thematisierens.

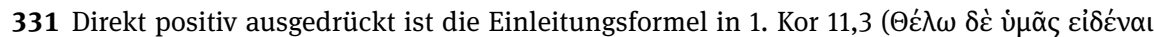

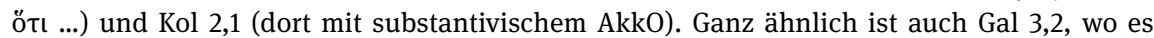

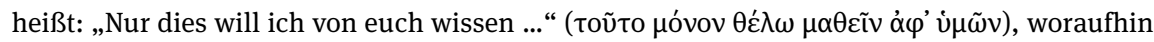

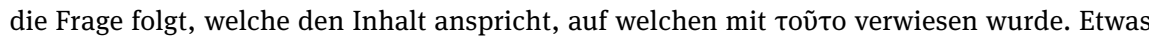
umständlicher, aber doch vergleichbar, ist Kol 1,27, wo in einem Relativsatz an die Heiligen aus V. 26 angeknüpft wird: „die wollte Gott wissen lassen, was der Reichtum ... [ist]“ (oî́ ñ $\theta \varepsilon ́ \lambda \eta \sigma \varepsilon v$

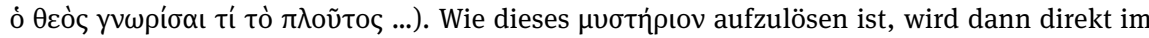

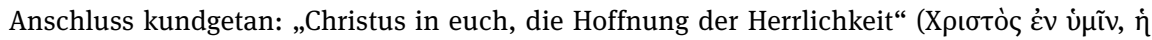

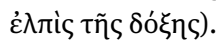

332 Es stimmt schon, dass dies eine „nachdrückliche Forderung“ ist (Schrage, Brief II, 445). Die nicht-negierten Belege, die hierfür aber in der Regel angegeben werden, sind wenig aussagekräftig. Zumindest kontextuell (V. 21) ist das Gemeinte klar. Die Negation im Griechischen bleibt aber bisher zu wenig untersucht. 


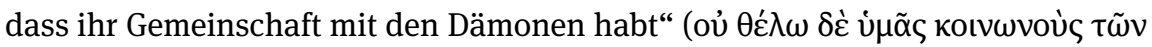

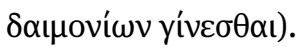

Und in 1. Kor 16,7 erklärt Paulus, weshalb er vorhat (V. 6), sich einige Zeit in Korinth aufzuhalten: „denn ich will euch jetzt nicht auf der Durchreise sehen“

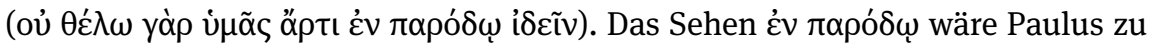
wenig, wie der Fortgang zeigt, in welchem ein alternatives - aus seiner Sicht bes-

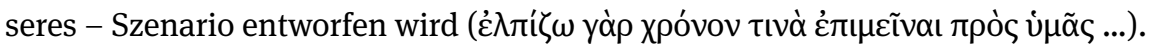
Man könnte daher mit gutem Recht übersetzen: ,ich will nicht, dass ich euch nur

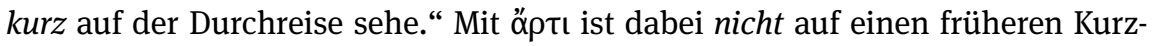
besuch hingewiesen. ${ }^{333}$ Vielmehr ist gemeint: „Wenn ich euch jetzt sehen wollte, so ginge dies nur auf der Durchreise - und das möchte ich nicht." Es ist also hier durchaus ein recht komplexes Protonarrativ ent- und dann verworfen. Die NIV löst das Gemeinte gut auf: „For I do not want to see you now and make only a passing visit.“

Ein recht konkreter zukünftiger Bezug dürfte auch in 2. Kor 5,4 vorliegen, wo in einem Kausalsatz gesagt ist: „... weil wir nicht entkleidet werden möchten“

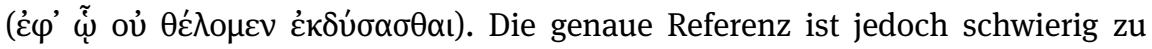
bestimmen, da diese Aussage in einem der ,schwierigsten [Texte] im 2Kor und im ganzen Neuen Testament“ erscheint. ${ }^{334}$

Wie man die verwobene Metaphorik von Behausung und Bekleidung interpretiert, hängt hier zu großen Teilen vom gewählten Grundansatz in der Auslegung des Briefes ab. ${ }^{335}$ Ein plausibler Ansatzpunkt bietet sich jedoch in 5,2 wo das gegenwärtige Seufzen im irdischen Zelt aus V. 1 mit dem Sehnen danach, „unsere

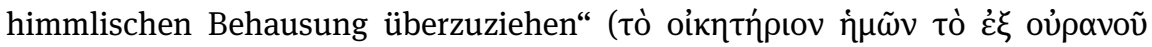

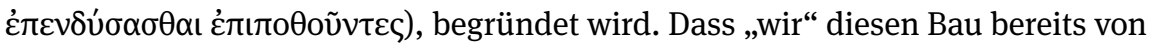

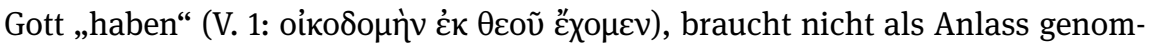
men zu werden, eine Vordatierung der Verleihung des Auferstehungsleibes (etwa auf den Todeszeitpunkt) vorzunehmen, sondern betont schlicht die bei Gott bereits bestehende Realität des eschatologisch erwarteten neuen Leibes. ${ }^{336}$

Entsprechend ist auch 2. Kor 5,3 am plausibelsten vor dem Hintergrund eines Kontrastes von gegenwärtigem und zukünftigem Leib zu lesen: Mit dem himmlischen Bau erst einmal angekleidet, werde man nicht „nackt“ befunden werden. Unabhängig davon, wie genau man die Stoßrichtung des Verses in der konkreten Situation mit den Korinthern verortet, wird man hier doch am ehesten eine

333 Richtig Schrage, Brief IV, 439.

334 Schmeller, Brief I, 285.

335 Für eine Übersicht über die Optionen siehe Schmeller, Brief I, 285.

336 So ganz richtig Wright, Resurrection, 368. 
Wertschätzung für den Zustand als Auferstandene gegenüber einem leiblosen und damit unvollständigen Zwischenzustand herauslesen dürfen. ${ }^{337}$

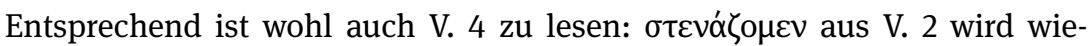
derholt und die dort zum Ausdruck kommende Sehnsucht des Überkleidetwer-

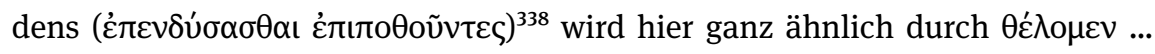

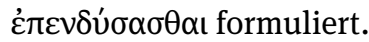

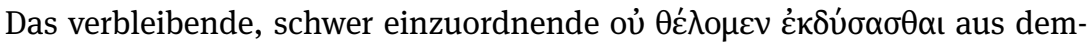
selben Vers muss dann wohl in der Tat auf den Tod vor der Parusie bezogen werden. Das Argument, dass die darin zum Ausdruck kommende Todesfurcht kaum eine angemessene Interpretation darstelle, erübrigt sich, wenn man hier einen effektiven Aorist annimmt und so einen Fokus auf die Nacktheit wie in V. 3 erhält: Es ist nicht so das Sterben, vor welchem sich Paulus fürchtet, sondern das unangenehme Resultat, dann eines Körpers entledigt zu sein. Paulus blickt also voraus auf den Zeitpunkt des Sterbens ${ }^{339}$ und bringt zum Ausdruck, dass die direkte Transformation in den erwarteten Auferstehungsleib vorzuziehen wäre. Dass die „Zerstörung“ der gegenwärtigen Behausung vor der Parusie jedoch durchaus eine Möglichkeit ist, bringt der Konditionalsatz in 5,1 bereits zum Ausdruck (

In diesem Abschnitt stehen sich somit zwei Protonarrative gegenüber, deren Handlungen jeweils in derselben Endsituation aufgehen, wobei Paulus die Verwirklichung einer der beiden potenziellen Erzählungen dennoch deutlich vorziehen würde, da diese der gegenwärtigen Leidenserfahrung im irdischen Leib eine unmittelbare Herrlichkeitserfahrung im himmlischen Leib entgegensetzen würde.

Nachdem ein kurzer Blick auf das verneinte Wollen bei Paulus geworfen wurde, soll jetzt ein verwandtes Phänomen betrachtet werden - Verben des Wollens in Konditionalsätzen. Hier ist es jedoch eindeutig der mentale Zustand des Wollen selbst, dessen Tatsächlichkeit in Frage steht. Deutlich wird dies etwa in 1. Kor 10,27, wo Paulus den Fall erwägt, dass die Korinther von nichtchristlichen Mitbürgern zu einer Feierlichkeit mit Mahl eingeladen werden. ${ }^{340}$ Für diesen im Alltag durchaus erwartbaren Fall würde man eigentlich einen prospektiven

337 Vgl. Wright, Resurrection, 367. Ebenso Schmeller, Brief I, 296, auch wenn die Rede von einem durch Paulus „gefürchteten“ Zwischenzustand dem Vers zu viel Gewicht beimisst.

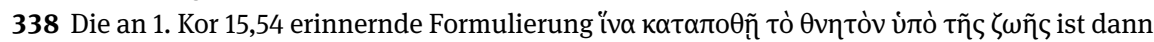
wohl eher konsekutiv zu verstehen.

339 Die Frage danach, wer hier in der ersten Person Plural alles (mit)gemeint ist, kann hier nicht erörtert werden. Zur Annahme eines rein „apostolischen“ Plurals im Zweiten Korintherbrief siehe jedoch Heilig, Paul's Triumph, Kapitel 6, Abschnitt 1.

340 Vgl. zum Abschnitt die von Arzt-Grabner, „Idol Meals“ zusammengestellte Evidenz. 


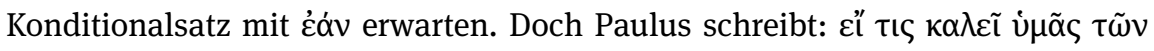

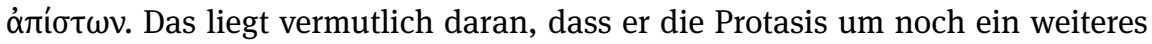
Verb ergänzt: „Wenn ihr von einem der Ungläubigen eingeladen werdet und ihr

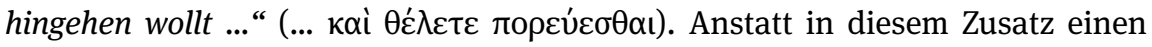
impliziten Ratschlag zu sehen, die Einladung auszuschlagen, ist es wohl eher so, dass Paulus hier die Entscheidung ganz beim Eingeladenen sieht und entsprechend auch für die gesamte Protasis den indefiniten Fall wählt. ${ }^{341} \mathrm{Ganz}$ ähnlich

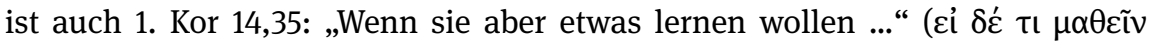
$\theta \dot{c} \lambda o v \sigma ı v)$. Inwiefern das jeweilige Protonarrativ hier eine für die Angeschriebenen relevante Handlung aufweist, liegt ganz an ihrem jeweiligen Wollen.

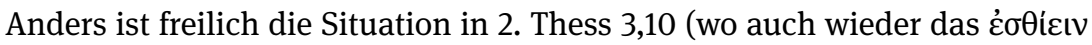
eine Rolle spielt, wenn auch unter ganz anderen Vorzeichen). Betont wird hier der notwendige Zusammenhang aus dem hypothetischen Fall des „Nicht-

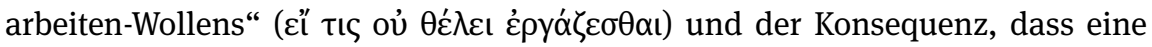

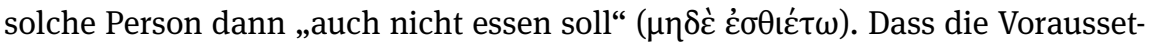
zung für dieses Protonarrativ tatsächlich erfüllt ist, wissen die Angesprochenen natürlich, und auch der Autor lässt dies unauffällig durchscheinen, wenn er im nächsten Vers $11 \mathrm{im} \mathrm{Hinblick} \mathrm{auf} \mathrm{tatsächlich} \mathrm{Gehörtes} \mathrm{( \alpha ُ \kappa oúo \mu \varepsilon v} \mathrm{...)} \mathrm{zwischen}$

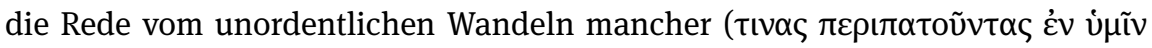

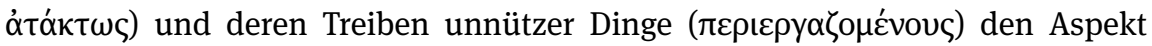
des unterlassenen „Arbeitens“ mit demselben Verb miteinfließen lässt ( $\mu \eta \delta \dot{\varepsilon} v$

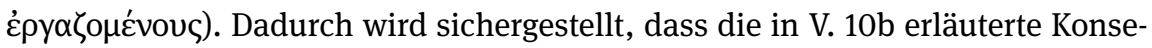
quenz als notwendige Fortsetzung der Handlung nun nicht mehr nur von einem hypothetischen Fall, sondern von der tatsächlichen Situation abhängig wird, ohne dass der Autor eine direkte Anordnung geben müsste. Das Protonarrativ stützt hier also eindeutig eine auf das Wollen und daraus folgende Handeln abzielende Kommunikationsintention. ${ }^{342}$

Die Möglichkeit, ein bestimmtes Handeln $\mathrm{zu}$ wollen, erscheint bei Paulus auch in einem prospektiven Konditionalsatz in 2. Kor 12,6: „Denn wenn ich

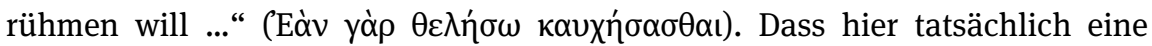
konkrete Absicht im Blick ist, deren Verwirklichung dann später im Vers explizit diserzählt wird ( $\varphi \varepsilon^{\prime} \delta$ o $\mu \alpha \iota \delta$ ś), wird in der Exegese in der Regel übersehen, ist aufgrund des Konditionalsatzes jedoch notwendig (siehe ausführlich unten, Abschnitt 5.2.2.3). Mit $\theta \varepsilon \dot{\lambda} \omega \omega+$ Infinitiv wird in 2. Kor 12,6 also im Konditionalsatz

341 Demgegenüber setzt 1. Kor 10,28 die Szene des Mahls bereits voraus und entsprechend kann Paulus hier wieder prospektiv den durchaus erwartbaren Fall erörtern, dass bei manchen dieser Einladungen das Wesen des Fleisches als ípó $\theta v \tau o v$ thematisiert werden könnte.

342 Vgl. auch die Analysen des Abschnitts 2. Thess 3,7b-8 in Kapitel 11, Abschnitt 2 und Kapitel 12 Abschnitt 2.3.2. 
ein potenzieller Handlungszusammenhang eingeleitet, der im Kontext wieder verworfen wird.

Der vom Wollen abhängige Verbinhalt wird auch an anderen Stellen ${ }^{343}$ kontextuell in Frage gestellt. So ist in 1. Tim 1,7 idiomatisch ${ }^{344}$ vom Wunsch

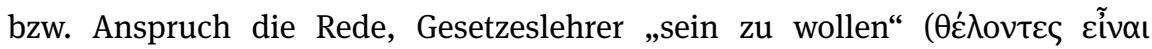

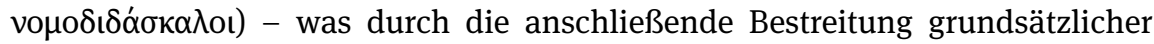

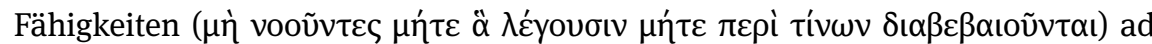
absurdum geführt wird.

Jedoch: In der Mehrzahl der Fälle, in welchen $\theta \varepsilon \dot{\lambda} \omega$ bei Paulus mit abhängigem Infinitiv erscheint, wird auf diese Weise - anders als in den bisher erläuterten Fällen - freilich ein tatsächlicher Wunsch zum Ausdruck gebracht. Dabei muss allerdings auch nicht immer ein konkretes zukünftiges Ereignis im Blick sein.

So fungiert in Röm 7,21 das Partizip von $\theta \varepsilon \dot{\lambda} \omega \omega$ als Attribut zum Personalpronomen und leitet einen die generelle Praxis betreffenden Wunsch ein: „... mir,

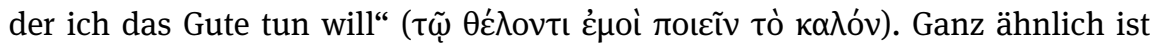
auch Röm 16,19, wo sich der allgemeine Wunsch allerdings auf den Lebenswandel anderer bezieht: ,ich will aber, dass ihr weise seid zum Guten, einfältig aber

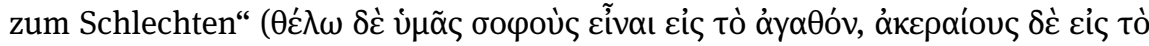
какóv).

Ähnlich allgemein scheint Paulus auch im Hinblick auf die Christen in Korinth $\mathrm{zu}$ formulieren, wenn er in 1. Kor 7,7 zum Ausdruck bringt, dass er „will,“ dass

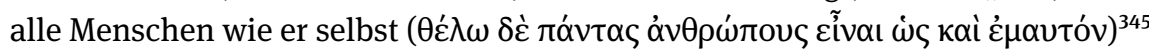

343 Zu Röm 9,22-23, wo $\theta \varepsilon ́ \lambda \omega$ als Partizip von einem finiten Verb im Aorist abhängig ist, welches zudem wohl Teil eines irrealen Konditionalsatzes ist, siehe oben, Kapitel 12, Abschnitt 8.3.

344 BDAG 3521,4 definiert ,to have an opinion“ und ergänzt die Glosse „maintain“ mit „,contrary to the true state of affairs." Der einzige neutestamentliche Beleg (2. Petr 3,5) scheint aber auch gut mit dem deutschen „wollen“ als intersubjektiv-referierend vereinbar (vgl. „Mein Freund will schneller laufen können als der Landesmeister“; Duden 826). Ausgedrückt wird dann lediglich, „dass der Sprecher den Inhalt des Inf. als eine Behauptung des Subj. hinstellt, deren Richtigkeit er bezweifelt“ $(e W D G)$.

345 Paulus fordert hier nicht in einem Desiderativsatz zu einem unverheirateten Leben auf. Er „wünscht“ dies nicht in diesem Sinn. Am ehesten noch nach Menge: „Ich möchte freilich wünschen ..." Eine Abschwächung im Sinn eines kontrafaktischen Desiderativsatzes ist nicht angemessen („Ach, wären doch nur alle Menschen wie ich!“). Denn es handelt sich hier in der Tat um ein Wollen, das Paulus auch in der Gegenwart antreibt - das Gewollte ist zumindest im

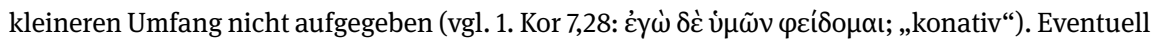
ist hier auch an einen zeitlich begrenzten Wunsch zu denken: Für den Moment (d. h., solange die „gegenwärtige Not“ anhält) will Paulus in der Tat ein entsprechendes Handeln umgesetzt sehen (und hätte dies nicht nur ,am liebsten, “ wie die NGÜ formuliert). Vgl. grundsätzlich zum Präsens Robertson, Grammar, 923. 


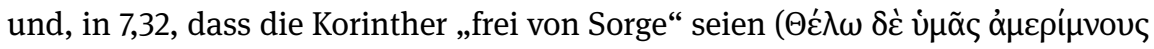

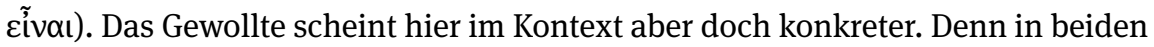
Fällen steht schließlich die recht konkrete Eheproblematik im Hintergrund. Im ersten Fall ist, wie der Fortgang des Verses zeigt, konkret an das $\chi \alpha \dot{\alpha} \rho \sigma \mu \alpha$ gedacht, welches Paulus erlaubt, unverheiratet zu bleiben (nicht aber an die Ehelosigkeit selbst). ${ }^{346}$ Und im zweiten Fall steht die Erläuterung in 7,28 im Hintergrund, wo den Heiratenden $\theta \lambda \tilde{\imath} \psi \mathrm{s}$, angekündigt wird. Zudem ist in V. 33 spezifischer die Rede davon, dass der Verheiratete um die Dinge der Welt besorgt sei ( $\mu \varepsilon \rho \mu \nu$ ạ $\tau \dot{\alpha}$

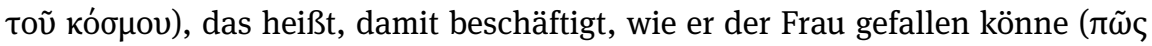

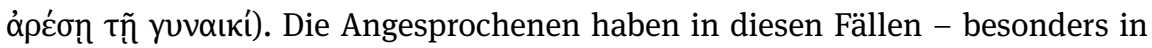
V. 32 - die genaue Referenz aus ihrer eigenen Erfahrung heraus zu ergänzen. Auch wenn die so gebildeten Protonarrative daher recht konkret gewesen sein dürften, könnte man, was das (fragmentarische) Erzählen des Paulus angeht, von einer recht großen „narrativen Distanz“ sprechen: Der Text selbst liefert recht wenige konkrete Hinweise, welche den Leser in seiner Imaginationstätigkeit einschränken würden. ${ }^{347}$

Ähnliches ließe sich auch über 2. Tim 3,12 sagen, wo vom „gottesfürchti-

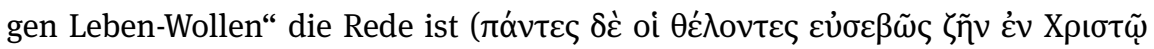

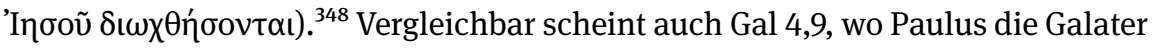
verwundert fragt: „Wie kann es sein, dass ihr euch wieder den schwachen und armseligen Elementen zuwendet?“ In einem Relativsatz unterstellt er ihnen im gleichen Zug: $:^{349}$ „... denen wollt ihr wieder von neuem dienen“ (oís $\pi \alpha \dot{\alpha} \lambda \iota$

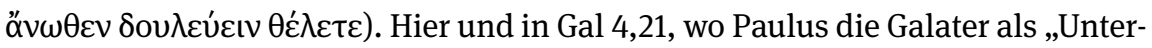

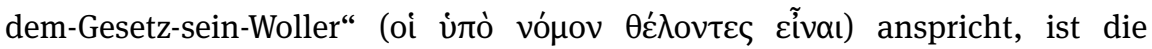
sprachliche Ausdrucksweise recht allgemein, auch wenn das ins Auge gefasste Geschehen sehr konkrete Ereignisse betrifft (vgl. etwa Gal 4,10 und mit Zukunftsbezug vor allem 6,12). An ein recht konkretes Geschehen dürfte auch in Gal 4,17 gedacht sein, wo Paulus behauptet, die Gegner wollten die Galater ,ausschlie-

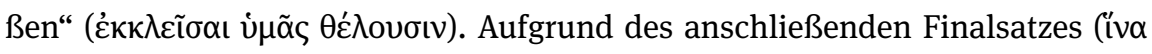

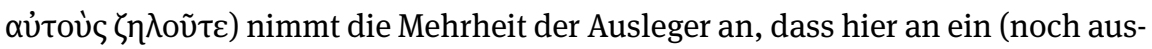
stehendes) völliges Abkapseln von Paulus und seinen Mitarbeitern gedacht ist,

346 Richtig Schrage, Brief II, 72.

347 Vgl. Köppe und Kindt, Erzähltheorie, 195. Vgl. etwa oben, Kapitel 6, Abschnitt 3.3.3.3. Zur Einordnung der Kategorie in den größeren Kontext des narratologischen „Werkzeugkastens“ siehe oben, Kapitel 3, Abschnitt 4.3.

348 Zur Abhängigkeit des futurischen Geschehens vom generell gebrauchten substantivierten Partizip siehe unten, Abschnitt 5.3.2.3.

349 In der NGÜ wird dies abgeschwächt zu einer weiteren Frage: „Wollt ihr ihnen wirklich von neuem dienen und ihre Sklaven sein?“ 
sodass die Galater stattdessen um die Gunst der Gegner - und nicht mehr um die der Gemeindegründer - buhlten. ${ }^{350}$

Der Grad an Konkretheit des Erzählten und die Anschaulichkeit des Erzählens müssen also nicht unbedingt korrelieren. Vielmehr kann Paulus in diesen Fällen voraussetzen, dass die Angesprochenen aufgrund ihres Welt- und Kontextwissens in der Lage sind, eigenständig sehr spezifische Protonarrative auszubilden. Dies erlaubt ihm, auf sehr allgemeine - zugleich aber stark wertende - Weise auf von sich oder anderen gewolltes zukünftiges Geschehen Bezug zu nehmen.

Relativ betrachtet anschaulicher ${ }^{351}$ sind wohl Texte wie etwa 1. Kor 14,19, wo Paulus mit $\theta \varepsilon \dot{\lambda} \lambda \omega$... n̆ ... ausdrückt, was er trotz seiner Befähigung zur Zungenrede

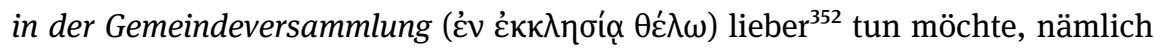
„fünf Worte“ mit dem Verstand reden, um andere zu unterweisen, als „zehntausend Worte“ in Zungenrede.

Vermutlich kann man eine ähnliche Einordnung auch für 1. Tim 2,4 vornehmen, wo der Retter-Gott aus V. 3 mit einem Relativsatz näher beschrieben wird: „welcher will, dass alle Menschen gerettet werden.“ Mit kaí wird dann ein weiterer Infinitiv angehängt, der aber wohl eher das Mittel spezifiziert, mit welchem dieser gewollte Zustand erreicht werden kann: „... und zur Erkenntnis der Wahr-

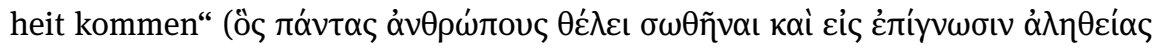
$\dot{\varepsilon} \lambda \theta \varepsilon \tilde{\imath} v)$.

Eine weniger weit gefasste Perspektive liegt in 1. Tim 5,11 vor, wo vom Abwei-

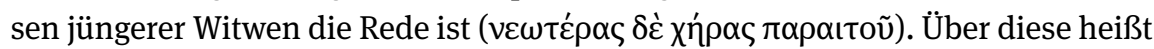

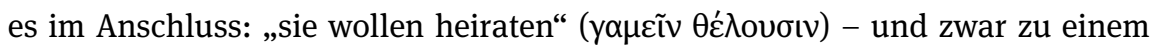
bestimmten, wenn auch verklausuliert ausgedrückten Zeitpunkt: „wenn sie Christus zuwider ihren sinnlichen Trieben folgen/wenn ihr sinnliches Verlangen

350 Vgl. NGÜ: „Sie wollen einen Keil zwischen euch und mich treiben, damit ihr euch dann um sie bemüht.“ Diese Interpretation ist jedoch nicht ohne Probleme. Denn der Wunsch des Ausschließens wird nicht mit einem (unerwünschten) Eifern der Galater für Paulus und seine Mitarbeiter begründet. Der Satzakzent in der deutschen Übersetzung (siehe oben, Kapitel 4, Abschnitt 8.1.3 zur Informationsstruktur) sollte vermutlich eher auf dem ,ihr“ liegen: Die Gegner bemühen sich zwar, aber eigentlich geht es ihnen nur darum, dass die Galater sich bemühen. Der Versuch, die Galater zu überzeugen, dass sie als Unbeschnittene nicht vollwertige Erben Abrahams seien, wird nach Paulus zwar mit viel Aufwand betrieben. Doch mit dieser Mühe wird den Galatern nichts abgenommen, sondern etwas aufgebürdet: Nun müssen nämlich sie etwas tun, sich beschneiden lassen.

351 Vgl. zum relativen Charakter der „Anleitung oder Einschränkung der sinnlichen Qualitäten von Vorstellungen“ Köppe und Kindt, Erzähltheorie, 195.

352 Vgl. BDR 480 ${ }^{5}$ und GGNT 252,26. 


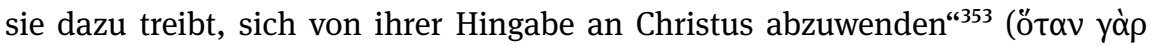

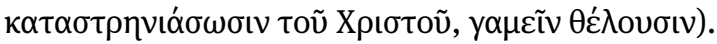

Man vergleiche auch 1 . Thes 2,18, wo Paulus versichert, dass er und seine

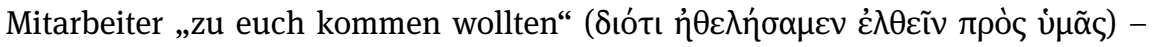

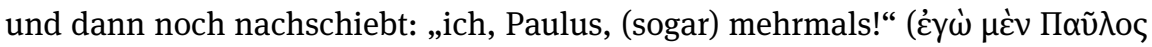

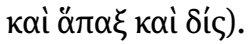

Auf sehr spezifisches Begehren, das auch einigermaßen anschaulich geschildert wird, wird in Gal 1,7 und Gal 6,13 Bezug genommen. Dabei wird im letzten Fall eigentlich sehr knapp formuliert: „sie wollen, dass ihr beschnitten werdet“

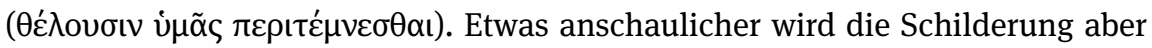
durch den angehängten Finalsatz: „damit sie sich eures Fleisches rühmen

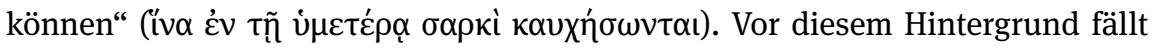
auf, dass im vorangehenden V. 12 dasselbe Geschehen durch das Relativpro-

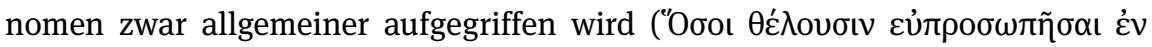

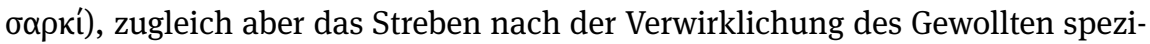

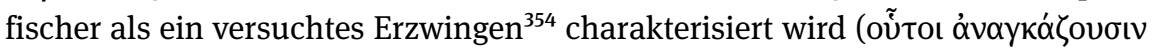

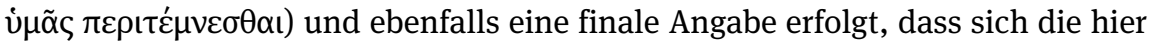
anonym Angeführten nämlich vor der Verfolgung um des Kreuzes Christi wegen

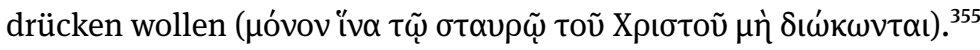

Eine ähnlich detaillierte Ausgestaltung findet sich in Gal 1,7, wo Paulus zunächst in Abrede stellt, dass die den Galatern von den Gegnern überbrachte Botschaft überhaupt ein „Evangelium“ sei: ${ }^{356}$ Alles was es gäbe, so meint Paulus

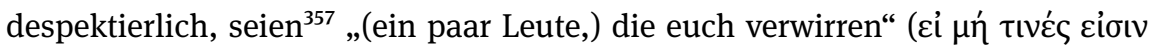

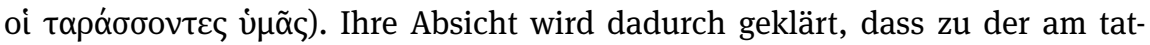
sächlich erzielten Effekt orientierten Charakterisierung ein weiteres Partizip

353 NSS 1119.

354 Vgl. oben, Kapitel 6, Abschnitt 3.4.1.1 zur „konativen“ Aspektnuance.

355 Auch hierbei ist natürlich wieder an spezifische soziale Konsequenzen gedacht, deren genauer Charakter allerdings von der Rekonstruktion des Konflikts in Galatien insgesamt abhängig ist. Vgl. für einen Vorschlag im Rahmen der kleinasiatischen Herrscherverehrung des römischen Kaisers Hardin, Galatians.

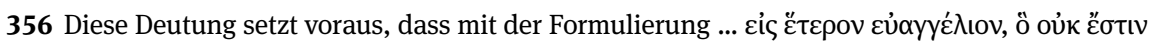
$\alpha \lambda \lambda$ o in Gal 1,6-7 keine Unterscheidung zwischen einem ,alternativen“ aber eben letztlich gar nicht „andersartigen“ Evangelium gemacht wird (Schröter, Jesus, 147-169), sondern der Gebrauch von

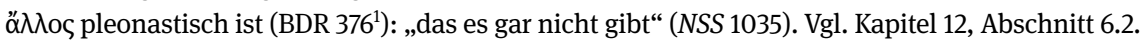

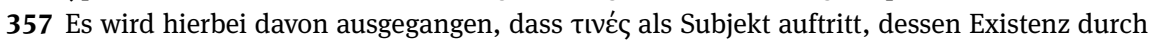
das Prädikat ausgesagt wird. Das Verb عijú hat also wohl keine Kopulafunktion (in der 3. Person Plural wohl kaum durch Akzentuierung angezeigt; persönliche Mitteilung von Heinrich von Siebenthal; zur 3. Person Sg. siehe GGNT 125b). Zum Artikel bei attibutiver Wortstellung und nicht determiniertem Bezugswort siehe GGNT 136a. 
angehängt wird: „... und (die) das Evangelium Christi verkehren wollen“ (... к $\alpha \grave{~}$

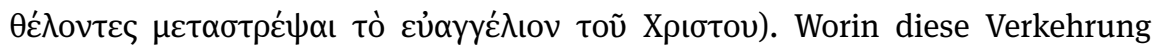
genau besteht, wann also der Wunsch zu seinem Ziel gekommen wäre, bleibt an dieser Stelle unerwähnt. Sehr ausführlich setzt sich Paulus aber in 1,7-8 mit diesem Protonarrativ auseinander: Gleich zwei Mal wird Gottes Verfluchung in Anspruch genommen, um jeglichen Handlungsstrang, der sich auf eine vom paulinischen Evangelium abweichende Botschaft stützt (also umso mehr auch deren Verdrehung in 1,6), im Keim zu ersticken.

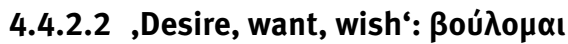

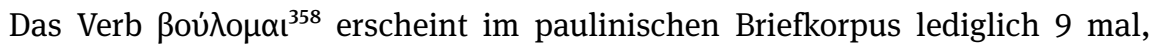

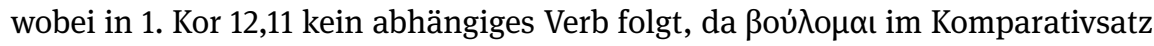

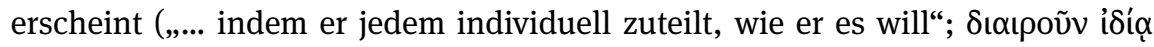

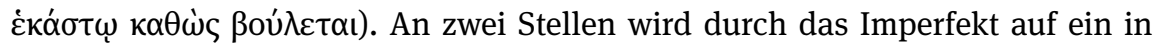
der Vergangenheit $\mathrm{zu}$ verortendes (und wie der Kontext zeigt: nicht erfülltes) Begehren verwiesen. ${ }^{359}$ In Phil 1,12 liegt eine auffällige Abwandlung der ansons-

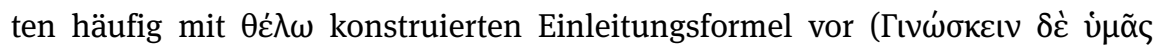

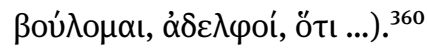

Die verbleibenden vier Formen entstammen allesamt den Pastoralbriefen. Ein durch den Durativ-Stamm als anhaltend markiertes „Insistieren“ wird in Tit

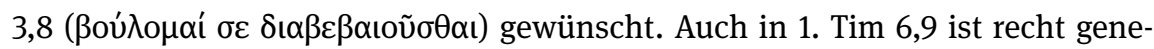
rell vom „Reichsein“ die Rede. Noch dazu wird dort (anders als in den anderen Belegen) kein Wollen des Briefschreibers ausgedrückt, sondern vielmehr zeitlos

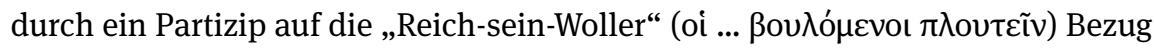
genommen. Ihr Schicksal wird ausführlich im Präsens beschrieben: Sie fallen in Versuchung, Verstrickung und viele und schändliche Begierden. Letztere werden wiederum im Präsens als „die Menschen“ in Verderben und Untergang versenkend beschrieben.

In einem zweifachen Schritt werden hier also jeweils umfassendere Ereignisbündel zusammengeschnürt und in einem grundlegendes Protonarrativ zusammengefasst. Dennoch ist auch hier durchaus an gegenwärtige und potenzielle zukünftige Ereignisse im erweiterten Leserkreis gedacht: Die allgemeine Schilderung dient als Hintergrund für die im Kontext aufscheinenden Verweise

358 LN 25.3: ,to desire to have or experience something, with the implication of some reasoned planning or will to accomplish the goal.

359 2. Kor 1,15.17 und wohl auch Phlm 13. Siehe hierzu Abschnitt 5.3.2.4.

$360 \mathrm{Vgl}$. für die verschiedenen Varianten Abschnitt 4.4.2.1. Zur Konstruktion mit verneintem

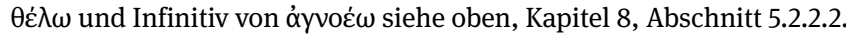




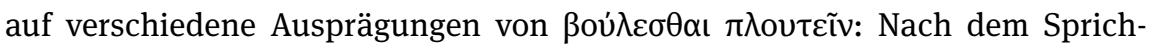
wort über die „Geldliebe“ in 6,10 ist von „manchen“ die Rede, die nach dieser

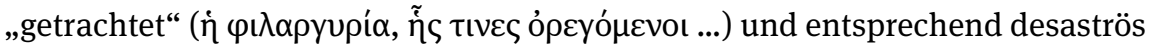
geendet hätten. Im Kontrast dazu soll Timotheus $(6,11)$ als „Mensch Gottes“361 sich von solchem Verhalten - und natürlich Trachten - fernhalten ( $\Sigma \dot{v} \delta \dot{\varepsilon}, \tilde{\omega}$

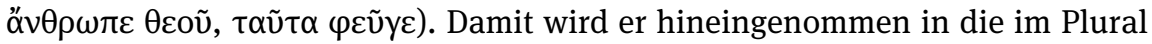
formulierte Absicht, ${ }^{362}$ es sich an Nahrung und Kleidung genügen $\mathrm{zu}$ lassen $(6,8$; vgl. die weitere narrative Stütze dieser Ebene in 6,7). Und implizit wird ihm dadurch auch der wahre „Profit“ aus V. 6 versprochen. Damit sollte jedoch auch klar sein, dass in V. 11 nicht einfach nur etwas über eine anonyme Menschengruppe oder spezifischer die damit angeblich gemeinten Irrlehrer gesagt ist. ${ }^{363}$ Der Gebrauch des Partizips erlaubt vielmehr jedem Leser individuell, sich innerhalb des Verhaltens zu verorten, das vom „Uns“ (V. 7-8), den „solchen“ (V. 10) und (potenziell) dem „Du“ (V. 11) aufgespannt wird. Den jeweiligen Handlungsfortgang ihres eigenen Protonarrativs können sie sich dann ohne Weiteres aus diesem Netz an Handlungsoptionen erschließen.

Ein konkretes Verhalten ist in 1. Tim 2,8 im Blick, wo der Wunsch geäußert

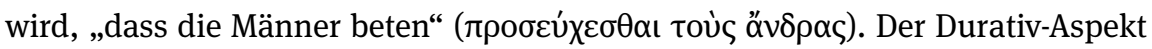
bringt hier eine iterative Aspektnuancierung hervor, ${ }^{364}$ welche durch die lokale

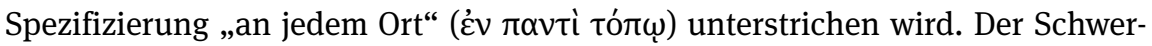
punkt des Wünschens liegt freilich auf der Art und Weise $e^{365}$ dieses Betens, welche durch ein modal gebrauchtes Partizip zum Ausdruck gebracht wird: ,indem sie

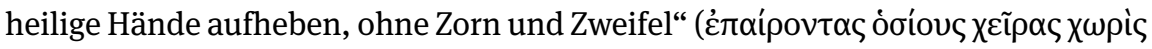

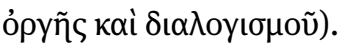

In 1. Tim 5,14 liegt dem dreifachen Wunsch für die „Jüngeren“ (d. h. jüngeren Witwen) vermutlich auch eine Ereignisfolge zugrunde: Sie mögen „hei-

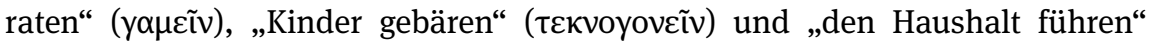

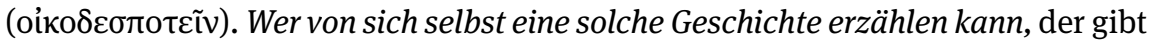
dem kritischen heidnischen Beobachter ${ }^{366}$ keine Gelegenheit, für „Lästerung“

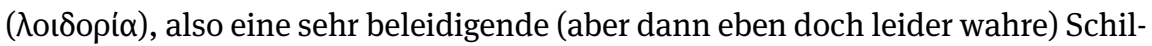
derung des unsozialen Verhaltens der christlichen Witwen.

361 Vgl. aber mit Recht Dibelius, Pastoralbriefe, 67 zur letztlich wohl breiteren Referenz.

362 Siehe hierzu bereits oben, Abschnitt 3.1.1.

363 Gegen Roloff, Brief, 337.

364 Vgl. NSS 1113.

365 Das zeigt die sehr ausführliche Gestaltung der an die Frauen gerichteten indirekten Auffor-

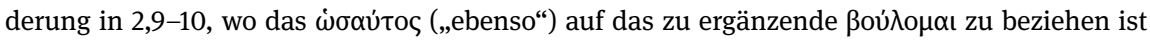
(NSS 1113).

366 Vgl. Roloff, Brief, 299-300. 


\subsubsection{Verben des Aufforderns (also Bittens und Befehlens)}

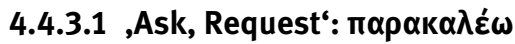

Im Folgenden sollen nun Verben des Aufforderns besprochen werden. Was konkreter die Verben des Bittens angeht, sind die Lexeme relevant, die von Louw-

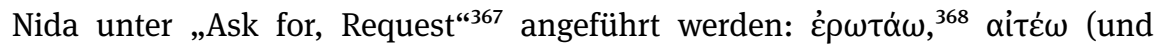

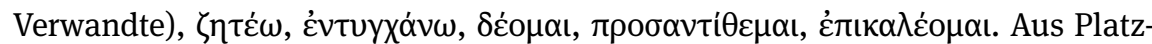
gründen beschränkt sich die Diskussion hier auf einige Beispiele zum Gebrauch von $\pi \alpha \rho \alpha \kappa \alpha \lambda \varepsilon \dot{\varepsilon} \omega$. Der Gebrauch mit Infinitiv auf der einen Seite und mit ivv $\alpha$-Satz zur Bezeichnung des Erbetenen auf der anderen Seite ist hier relativ ausgeglichen. ${ }^{369}$

Mit (a) Infinitiv konstruiert werden ${ }^{370}$ die eindringlichen Bitten, ${ }^{371}$ „die Körper

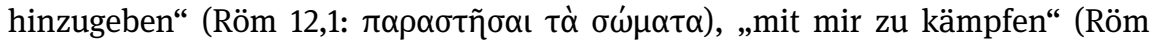

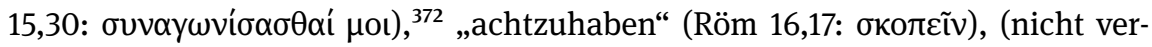

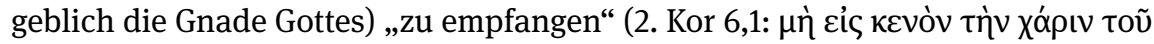

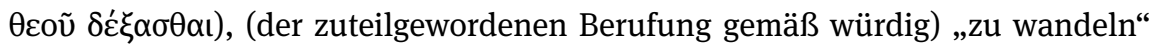

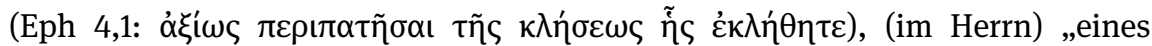

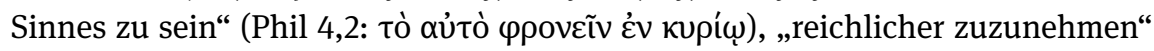

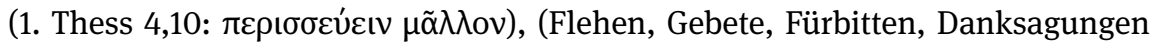

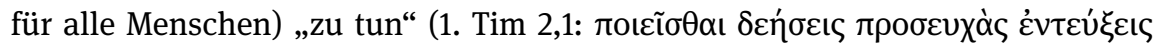

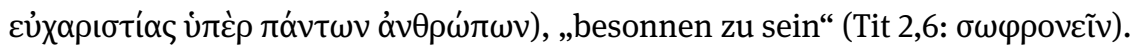

Der Gebrauch in 2. Kor 2,8 fällt vor diesem Hintergrund besonders auf, da hier kein generelles Verhalten im Blick zu sein scheint, sondern vielmehr ein einmaliger Beschluss in der Zusammenkunft der Gemeinde: Der Beschluss, der gefällt

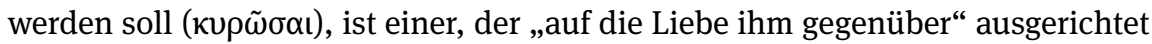

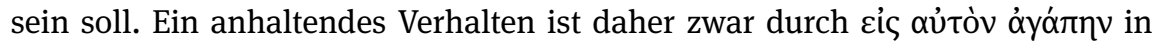

367 LN 33.161-177.

368 Siehe dazu den kurzen Exkurs weiter unten in diesem Abschnitt.

369 Die Konstruktion mit ö $\pi \omega \varsigma$ ist bei Paulus nicht belegt. Siehe aber Mt 8,34. Für die Einleitung eines selbstständigen Begehrsatzes (d. h. eines asyndetisch folgenden Imperativs; vgl. oben, Abschnitt 4.4.2.1) siehe 1. Kor 4,16 und Eph 4,1. Siehe auch 1. Thess 5,14 im Plural. Vgl. auch Hebr 13,22 und 1. Petr 5,1.

370 Vgl. außerdem die in der Vergangenheit lokalisierten Wünsche in 1. Thess 2,12 und 1. Tim 1,3. Vgl. hierzu grundsätzlich oben, Kapitel 11, Abschnitt 6 zu Vorausdeutungen auf die Vergangenheit des Erzählers.

371 Die Semantik des im Deutschen als Äquivalent oft verwendeten „Ermahnens“ ist nicht wirklich passend, um den Gedanken ,to ask for something earnestly and with propriety` (LN 33.168) zum Ausdruck zu bringen.

372 Siehe dazu oben, Kapitel 11, Abschnitt 8.8. 
den Blick gefasst, der aoristische Infinitiv bezeichnet jedoch erst einmal nur die von Paulus angeregte gemeinschaftliche Entscheidung für diesen Versöhnungsprozess. $^{373}$

Zur Konstruktion (b) mit abhängigem îvo-Satz sind folgende Bitten zu zählen: ${ }^{374}$ In 1. Kor 1,10 wird positiv gefordert, die Korinther mögen alle „dasselbe

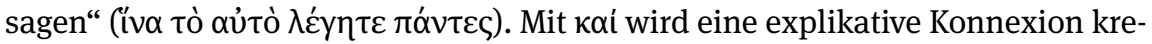
iert. ${ }^{375}$ Diese wiederum besteht aus einem Gefüge aus zwei Propositionen nach dem Muster Negativum-POSITIVUM: „Spaltungen“ soll es unter ihnen nicht

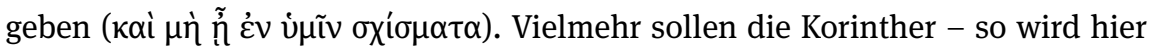
durch den seltenen Konjunktiv Perfekt betont - in den Zustand versetzt sein, in welchem sie „dieselbe Gesinnung und dieselbe Überzeugung“ aufweisen ( $\tilde{\eta} \tau \varepsilon \delta \dot{\varepsilon}$

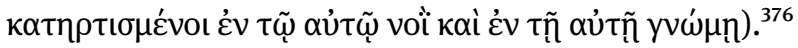

Was die Thessalonicher-Briefe betrifft, ist es in 2. Thess 3,12 das Essen des eigenen Brotes, welches durch ruhiges Arbeiten erworben werden soll, welches von $\pi \alpha \rho \alpha \kappa \alpha \lambda$ ũ $\mu \varepsilon v$ abhängig ist - und welches wiederum koordiniert ist mit dem gebieterischeren $\pi \alpha \rho \alpha \gamma \gamma \varepsilon \dot{\lambda} \lambda o \mu \varepsilon v$ (vgl. unten, Abschnitt 4.4.3.6). Ein weite-

373 Natürlich ist es nicht der Aspekt selbst, der ein „punktuelles“ Verständnis fordert. Dass eine solche Auffassung unhaltbar ist, lässt sich auch sehr schön an den hier zusammengestellten Beispielen zeigen. Röm 12,1 und 2. Kor 6,1 könnten eventuell noch in einen solchen Rahmen gepresst werden. In Röm 15,30 ist der komplexive Charakter des Aorists jedoch unleugbar, da

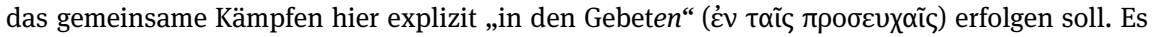
ist vielmehr die perfektive Betrachtung der telischen Situation, die ein solches Verständnis nahelegt. LN 70.6 grenzen eine weitere Bedeutung ab (,to cause someone to recognize the reality of something'). Es wäre dann eventuell nicht an den gemeinschaftlichen Beschluss gedacht, nun Liebe walten zu lassen (vgl. NSS 1002-1003), sondern an die eigentliche Ausübung dieser Liebe, die zwar ebenfalls auf ein Ziel hinauslaufen würde, jedoch durativer aufgefasst werden könnte. Die Rede von der mehrheitlichen Bestrafung in 2,6 scheint jedoch eher gegen diese Auffassung zu sprechen. Vgl. auch 1. Kor 5,3 (auch wenn nicht davon auszugehen ist, dass von derselben Person die Rede ist); siehe dazu Heilig, Paul's Triumph, Kapitel 4 und bereits oben, Kapitel 8, Abschnitt 3.3). Grundsätzlich ist die Existenz der von LN angenommenen Bedeutung (für die nur 2. Kor 2,8 als Beleg angegeben wird) zweifelhaft. Ein duratives Verständnis ließe sich also höchstens dann ableiten, wenn man von einem komplexiven Aorist und einer Serie an Entscheidungen für die Liebe ausgeht. Auffällig ist auf jeden Fall, dass im NT und der LXX das Verb nie im Durativ erscheint, um ein solches iteratives Entscheiden explizit zu kommunizieren.

374 Für Bitten, die in der Vergangenheit liegen, siehe grundsätzlich oben, Kapitel 11, Abschnitt 6 zu Vorausdeutungen auf die Vergangenheit des Erzählers. Siehe etwa 1. Kor 16,12 und vgl. die etwas anders gelagerte Situation in 2. Kor 8,6.

375 Vgl. GGNT 341a.

376 Zum umschriebenen resultativen Konjunktiv vgl. GGNT 203a unter c). Für die Paulusbriefe wird hier an Vergleichsstellen angeführt: 2. Kor 1,9, 9,3, 2. Tim 3,17. 
rer paulinischer Beleg für $\pi \alpha \rho \alpha \kappa \alpha \lambda \varepsilon \dot{\varepsilon} \omega$ mit îv $\alpha$ findet sich in 1. Thess 4,1. ${ }^{377}$ Auch hier tritt $\pi \alpha \rho \alpha \kappa \alpha \lambda \varepsilon \dot{\varepsilon} \omega$ nicht alleine auf, sondern in der Verbindung $\varepsilon \rho \omega \tau \tilde{\omega} \mu \varepsilon v \dot{v} \mu \tilde{\alpha} \varsigma$

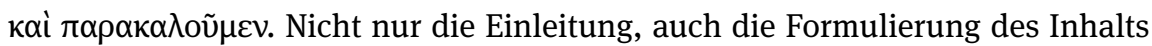
der Bitte in 1. Thess 4,1 ist bemerkenswert: Der iv $\alpha$-Satz wird sofort von einem Komparativsatz unterbrochen: „damit - wie ihr von uns empfangen habt ... “ (iv $\kappa \alpha \theta \dot{\omega} \varsigma \pi \alpha \rho \varepsilon \lambda \alpha \dot{\beta} \varepsilon \tau \varepsilon \pi \alpha \rho$ ' sierten Empfangens ist nun ein indirekter Fragesatz: ${ }^{378}, \ldots$ wie ihr wandeln und

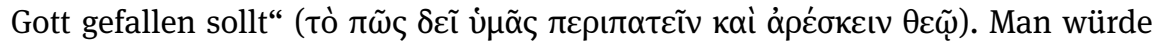
nun erwarten, dass sich das Korrelat anschließt (,Wir bitten euch ... dass ihr wie ihr empfangen habt ... - so jetzt auch ..."). Stattdessen aber wird ein weite-

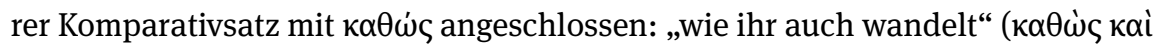
$\pi \varepsilon \rho ı \pi \alpha \tau \varepsilon \tau \varepsilon)$. Dann erst schließt sich die eigentliche Aufforderung an: „dass ihr mehr und mehr zunehmt“ ('iv $\alpha \varepsilon \rho \rho \sigma \sigma \varepsilon u ́ \eta \tau \varepsilon \mu \tilde{\alpha} \lambda \lambda o v)$.

Die Aufforderung ist also eingebettet in ein Protonarrativ, welches folgende Ereignisse beinhaltet:

(1) Eine direkte, an Paulus und seine Mitarbeiter gerichtete Frage.

(2) Die im dafür typischen Lexem $\pi \alpha \rho \alpha \lambda \alpha \mu \beta \alpha \dot{\alpha} \nu \omega$ ausgedrückte Instruktion.

(3) Damit einhergehend natürlich den in der Vorvergangenheit zu lokalisierenden Wunsch, die Thessalonicher möchten entsprechend handeln.

(4) Die sich anschließende Verwirklichung dieses Wunsches.

(5) Eine neue Bitte, deren Inhalt durch die pleonastische Formulierung ${ }^{379}$ mit $\pi \varepsilon \rho ı \sigma \sigma \varepsilon v ́ \omega+$ $\mu \tilde{\alpha} \lambda \lambda$ ov als Fortführung und Steigerung des bisherigen Verhaltens qualifiziert wird. ${ }^{380}$

377 Das Verb ع́ $\rho \tau \tau \alpha \dot{\omega} \omega$ (LN 33.161: ,to ask for, usually with the implication of an underlying question') erscheint außer an dieser Stelle in den Paulusbriefen nur noch an drei anderen Stellen: In Phil 4,3 bittet Paulus den anonymen Gefährten ( $\sigma u ́ \zeta$ ıуo ) den in 4,2 genannten Frauen „beizustehen.“ Dieser Akt wird interessanterweise durch einen neu einsetzenden medialen Imperativ zum Ausdruck gebracht ( $\sigma v \lambda \lambda \alpha \mu \beta \alpha$ ovov) und nicht durch eine abhängige Infinitiv- oder iva-Konstruktion. Bei der (im Plural formulierten) Bitte um das „Anerkennen“ der „unter euch Arbeitenden, unter euch Vorstehenden im Herrn und euch Ermahnenden“ in 1. Thess 5,12 ist hin-

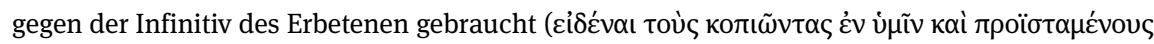

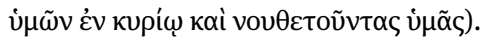

Das $\dot{\varepsilon} \rho \tau \tilde{\omega} \mu \varepsilon v$ in 2. Thess 2,1 wird im Folgevers V. 2 durch das Akkusativobjekt ergänzt. Während der Infinitiv mit عiৎ tó in der Regel eine konsekutive oder finale Umstandsangabe/-ergänzung liefert (GGNT 226), liegt hier der seltene Fall vor, dass die Konstruktion (hier verneint durch $\mu$ '́) das Objekt bereitstellt: „dass ihr euch nicht schnell in eurem Sinn erschüttert werdet

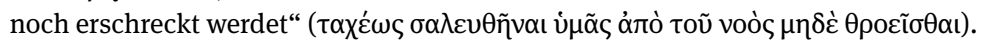

378 GGNT 273b.

379 Siehe GGNT 138b.

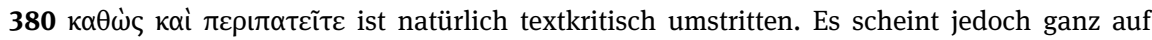

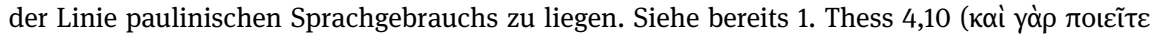




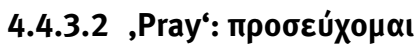

Bevor wir uns den Verben des Befehlens zuwenden können, muss im Zusammenhang mit dem Themenkomplex der Verben des Bittens noch ein weiterer Aspekt kurz angesprochen werden: das Gebet. Als im weitesten Sinne „Reden mit Gott“381 schließt es natürlich auch ihm gegenüber geäußerte Bitten ein. Dementsprechend kann etwa $\pi \alpha \rho \alpha \kappa \alpha \lambda \varepsilon \dot{\varepsilon} \omega$ auch mit Gott/dem Herrn als direktem Objekt auftreten. ${ }^{382}$

In Kol 1,9 haben wir eine Kombination aus einem primär die Äußerung selbst ausdrückenden Verb $(\pi \rho \circ \sigma \varepsilon v ́ \chi 0 \mu \alpha \mathrm{s})^{383}$ und einem spezifischer die Erwar-

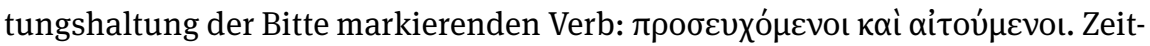
lich wird diese Bitte in die Gegenwart geholt, indem die Autoren versichern,

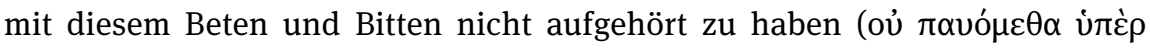

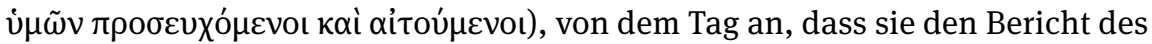

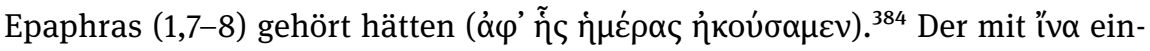
geleitete Inhalt wird dann passivisch wiedergegeben: „dass ihr erfüllt würdet

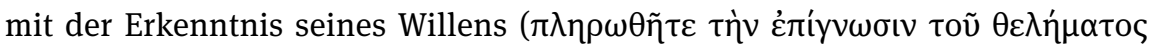

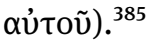

Nun ist es so, dass auch allgemeine Verben des „Sagens“ wie $\lambda \dot{\varepsilon} \gamma \omega$ zum Teil in bestimmten Kontexten abhängige Begehrsätze einleiten können. ${ }^{386} \mathrm{Im}$ corpus Paulinum geschieht Ähnliches auch einige Male mit dem auf die Äußerung

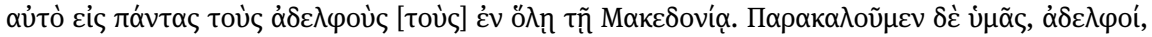

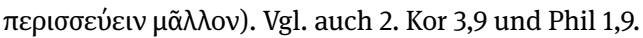

381 Vgl. LN 33.178.

382 Vgl. 2. Kor 12,8, wo auf eine in der Vergangenheit liegende - und abgewiesene - Bitte Bezug genommen wird. Siehe dazu oben, Kapitel 12, Abschnitt 2.3.2.

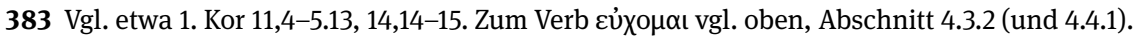
384 Auch Eph 1,15 greift temporal auf dieses „Hören“ als Anfangspunkt des Gebetes zurück (al-

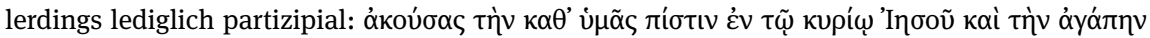

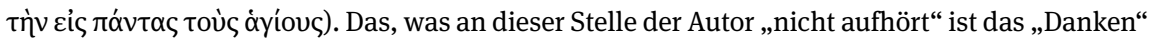

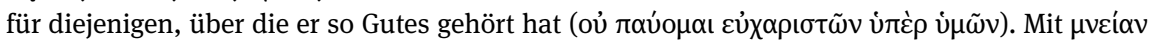

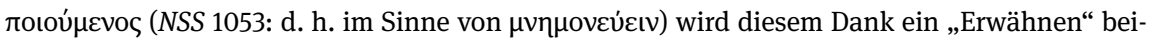

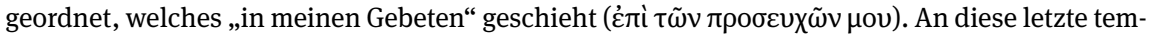
porale/modale Angabe knüpft dann wohl das ǐv $\alpha$ an, welches V. 17 und letztlich den ganzen Satz bis V. 23 einleitet. Eine noch losere Anbindung eines abhängigen Begehrsatzes mit ìv findet sich

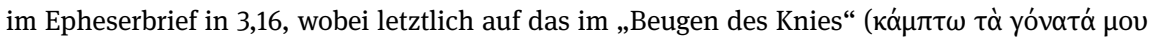

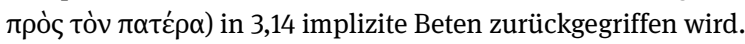

385 Es ist daher nicht ganz korrekt, hier ohne Einschränkung von einer „Fürbitte“ zu sprechen (Schweizer, Brief, 40). Es ist ein Gegenwartsbezug gegeben, doch was den sprachlichen Akt des Briefschreibens angeht, liegt hier lediglich ein Gebetsbericht vor.

386 Vgl. GGNT 218a und f. 
fokussierenden $\pi \rho \circ \varepsilon \varepsilon u ́ x о \mu \alpha$, wenn dieses nicht (anders als in Kol 1,9) durch ein weiteres, die Sprechhandlung betonendes Verbs des Bittens hervorgehoben wird.

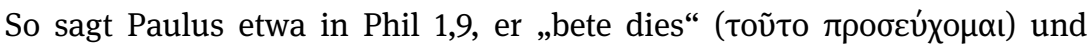
fügt dann mit ǐva an: „dass eure Liebe noch mehr und mehr überreich werde in

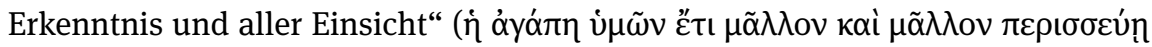

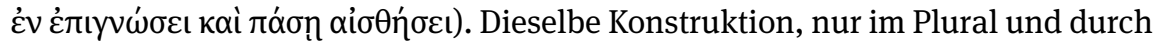

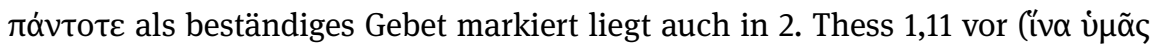

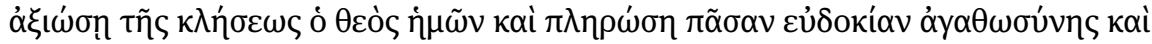

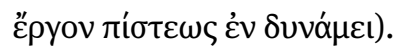

Nicht selten ist der Akt des Betens in solchen Fällen zukünftig oder poten-

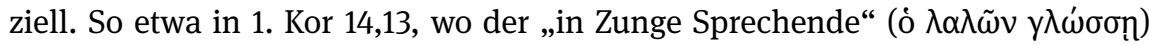
durch einen Imperativ zu einem Gebet aufgefordert wird, welches den Inhalt hat,

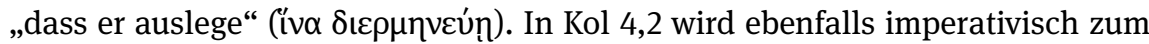

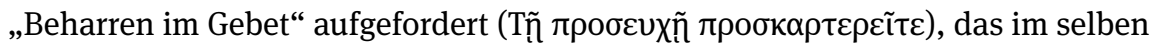

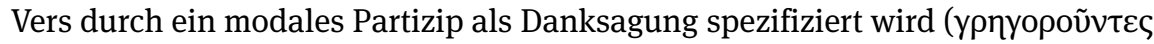

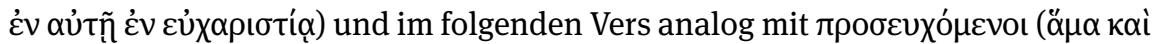

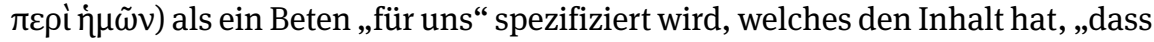

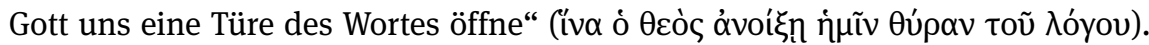

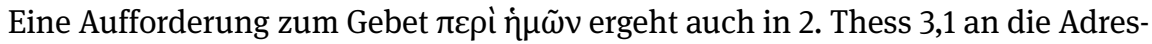
saten, welches zum Gegenstand die Bitte hat, „dass das Wort des Herrn laufe und

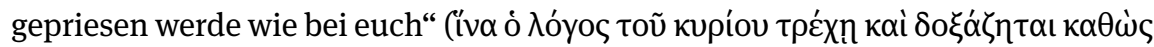

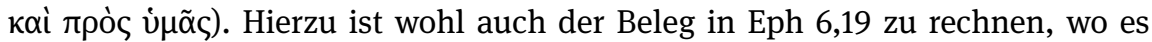

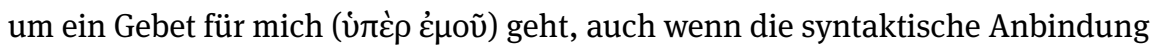
etwas lose ist: ${ }^{387}$ „dass mir Rede gegeben werde, wenn ich den Mund öffne“ (iv

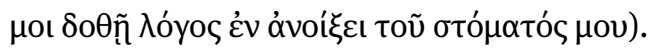

\subsubsection{3 ,Command, order': Allgemeines}

Nach diesem Überblick über abhängige Begehrsätze und Infinitive nach Verben des Bittens, soll nun noch ein schlaglichtartiger Überblick über vergleichbare Konstruktionen nach Verben des Befehlens gegeben werden.

Natürlich ist zu beachten, dass auch Verben, deren Semantik dem Bitten zuzuordnen ist, in bestimmten Kontexten durchaus die Bestimmtheit eines

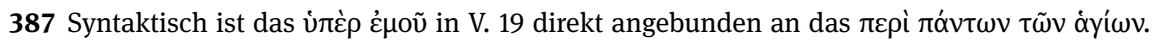

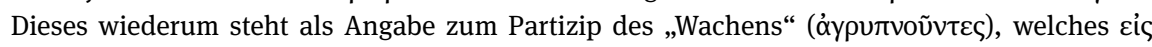

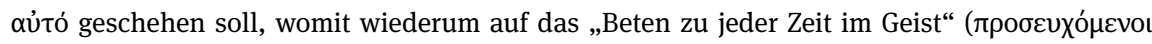

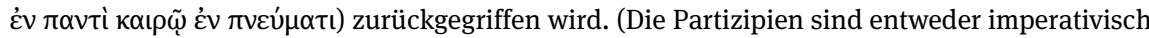
gebraucht oder von $\delta \varepsilon \dot{\varepsilon} \xi \sigma \theta \varepsilon$ in V. 17 abhängig.) 
,Befehls‘ aufweisen können. ${ }^{388}$ Andererseits sind „Empfehlungen,“ die als solche schwächer als die Befehle sind, bei Paulus nicht durch Lexeme mit dieser einleitenden Funktion belegt. ${ }^{389}$

Auch Verben, die lediglich den Aspekt der Äußerung kommunizieren (wie

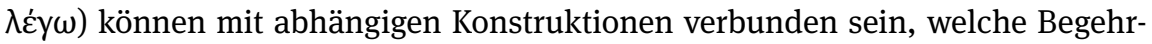
sätzen entsprechen. ${ }^{390}$ Dies gilt auch für Verben, die einen zusätzlichen Aspekt zum Ausdruck bringen, der mit Befehlen häufig verbunden, aber diesen nicht inhärent ist. Dies ist wohl der Fall bei $\delta \iota \alpha \mu \alpha \tau u ́ \rho o \mu \alpha$ in 1 . Tim 5,21, denn im folgenden Vers 22 wird mit einem normalen Imperativ fortgefahren. ${ }^{391}$

Hier sollen allerdings diejenigen Verben im Zentrum stehen, die von LouwNida tatsächlich unter der Sub-Domäne „Command, Order“392 gelistet werden:

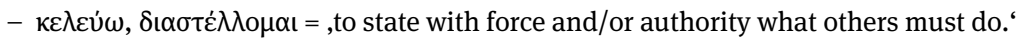

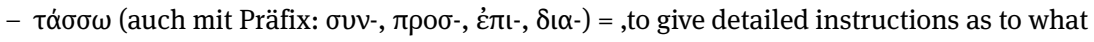
must be done.'

- $\dot{\alpha} \pi \alpha y=\dot{\varepsilon} \lambda \lambda \omega, \pi \alpha \rho \alpha y=\dot{\varepsilon} \lambda \lambda \omega=$,to announce what must be done.

- $\varepsilon \dot{\varepsilon} \tau \dot{\varepsilon} \lambda \lambda \mathrm{o} \mu \alpha \mathrm{l}=$,to give definite orders, implying authority or official sanction.

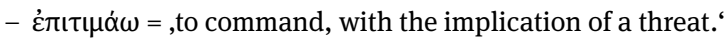

388 Trotzdem ist die semantische Unterscheidung wahrzunehmen, gerade um die rhetorische Funktionalisierung in diesen Fällen angemessen herausarbeiten zu können.

389 Vgl. LN 33.343-346: „Recommend, Propose.“

390 Vgl. GGNT 218f. Von diesem Gebrauch zu unterscheiden sind natürlich Fälle, in denen $\lambda \varepsilon ́ y \omega$ einen selbstständigen Begehrsatz einleitet. So etwa in 2. Kor 11,16: „Wiederum sage ich“ (Пá $\lambda$ เv

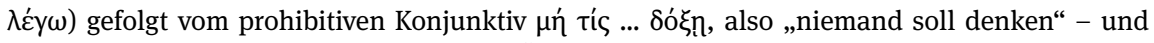
zwar: „dass ich ein Tor bin“ ( $\mu \varepsilon$... ö $\varphi \rho o v \alpha$ عĩvol).

391 Zugleich verdeutlicht dieses Beispiel, dass die vorgenommene definitorische Grenzziehung bei den betreffenden Verben nicht immer einfach ist. Der mit i ivo eingeleitete Nebensatz beinhaltet das „Befolgen“ ohne Vorurteil, was konkret auch beinhaltet, niemanden zu begünstigen (ivv $\alpha$

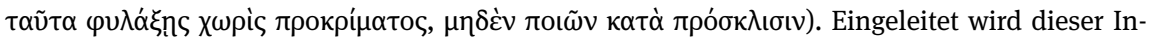

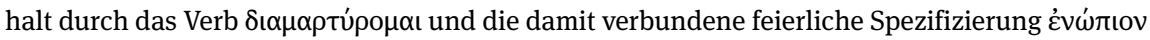

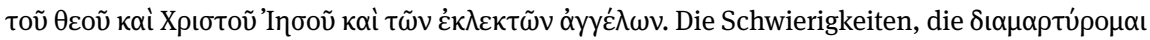
Lexikographen bereitet, lässt sich bereits daran erkennen, dass englische Übersetzungen mehrheitlich „to charge“ (d. h. „beauftragen“) als Äquivalent wählen, welche die meisten deutschen Übersetzungen vom „Ermahnen“ sprechen. Die Glossen von BA, „beschwören, dringend zureden“ (BA 273) welche vor allem die Eindringlichkeit der Äußerung zu betonen scheinen, werden bei BDAG 1878,2 zu einer recht spezifischen Definition umgestaltet (,to exhort with authority in matters of extraordinary importance, freq. w. ref. to higher powers and/or suggesiton of peril'), welche jedoch nicht so recht zur Bandbreite der dafür gebotenen Glossen passen möchte (,solemnly urge, exhort, warn“). Die von BDAG angegebene Grundbedeutung (,to state something in such a way that the auditor is impressed with its seriousness') ist andererseits gut kompatibel mit der von Louw-Nida angegebenen Unterbedeutung ,to be emphatic in stating an opinion or desire' (LN 33.319).

392 LN 33.323-332. 


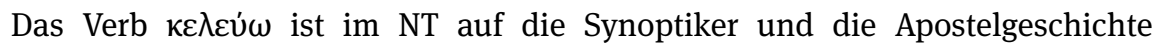
beschränkt. ${ }^{393}$ Ähnliches gilt für $\delta ı \alpha \sigma \tau \varepsilon ́ \lambda \lambda o \mu \alpha \iota,{ }^{394}$ welches in den Briefen nur noch in Hebr 12,20 (als Einleitung für das Gebot in Ex 19,13) erscheint. Das Verb

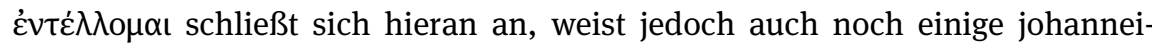

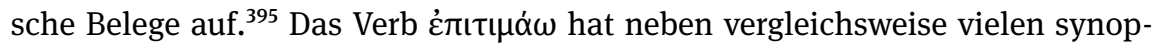
tischen Belegen in der Briefliteratur auch einen Auftritt in Jud 9 - allerdings in anderer, das „Schelten“ ausdrückender, Bedeutung. ${ }^{396}$ Dies gilt wohl auch für das einzige Auftreten im paulinischen Briefkorpus, für 2. Tim 4,2, wo es in einer Reihe von Imperativen erscheint: кń $\pi \alpha \rho \alpha \kappa \alpha ́ \lambda \varepsilon \sigma o v$...

Wie ist die Situation bei đó $\sigma \sigma \omega$ mit und ohne präpositionalem Vorderglied? ${ }^{397}$ Die Form mit ouv- erscheint im NT überhaupt nur bei Matthäus. ${ }^{398}$ Die Form mit

393 Es taucht mit auffälliger Häufigkeit im matthäischen Sondergut auf (z. B. Mt 18,25). Vgl. jedoch auch Mt 14,18, wo bei Mk 6,39 غ̇лıтó $\sigma \sigma \omega$ steht und Lk 9,14-15 den direkten Befehl umschreibt. Vgl. auch Mt 14,9 // Mk 6,26 und Mt 8,18 / Mk 4,35 (keine Entsprechung bei Lukas). An einer Stelle (Lk 18,40) hat Lukas dieses Verb, während bei Mt 20,32 das markinische „er sprach:

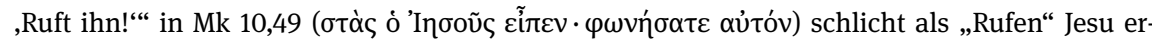

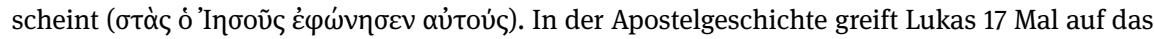
Verb zurück.

394 Die synoptischen Belege sind wiederum interessant. Auffallend ist Mt 16,20 für ein Gebie-

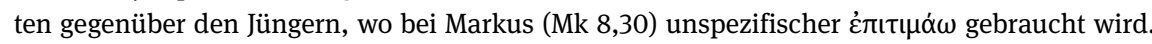
Auch Lukas verstärkt den markinischen Ausdruck, jedoch nicht durch Ersatz des Verbs, sondern durch zusätzliches $\pi \alpha \rho \alpha y y \dot{\lambda} \lambda \omega$ (Lk 9,21). Abgesehen von diesem matthäischen Gebrauch gegen Markus, liegt sonst markinischer Vorzug von $\delta \iota \alpha \sigma \tau \dot{\varepsilon} \lambda \lambda$ o $\mu \alpha$ เ vor: Das Schweigegebot aus Mk 5,43

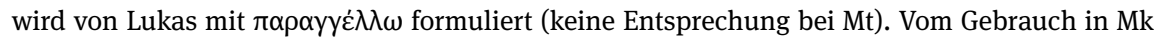
8,15 mit modalem $\lambda \varepsilon ́ \gamma \omega \nu$ wird lediglich das letztere Verb bei Mt 16,6 und Lk 12,1 übernommen. Andersherum wird das Verb aus Mk 9,9 in Mt 17,9 durch das die direkte Rede einleitende Partizip

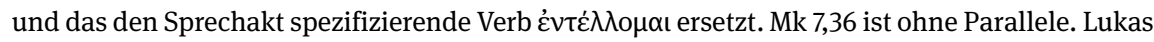
gebraucht das Verb jedoch in Apg 15,24.

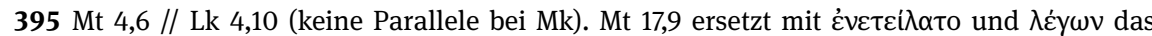

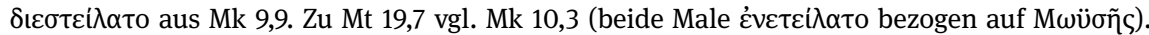
Mt 28,20 gehört zum matthäischen Missionsbefehl. Siehe jedoch die interessante Parallele in Apg 1,2 (das einzige weitere Vorkommnis in der Apostelgeschichte ist Apg 13,47). Neben den drei Vorkommnissen in Joh 14-15 (14,31, 15,14.17) erscheint das Verb - ebenfalls auf Mose bezogen innerhalb der Ehebrecherin-Perikope in 8,5. Die beiden Vorkommnisse im Hebräerbrief haben alttestamentliche Bezüge: In Hebr 9,20 erscheint es im Zitat aus Ex 24,8, wo jedoch $\delta ı \alpha i ́ \eta \mu$ verwendet wird. Hebr 11,22 paraphrasiert Gen 50,25, wo ò $\kappa$ кi $\zeta \omega$ verwendet wird.

396 LN 33.149: ,to express strong disapproval of someone.' Zur Traditionsgeschichte siehe Frey, Brief, 84-85.

397 Vgl. GGNT 369.

398 In Mt 21,6 ist Mk 11,4-6 verkürzt wiedergegeben. Dort und in Lk 19,32-34 wird die Ausführung des „Befehls“ selbst geschildert und nicht nur das Vorfinden des Angekündigten. Der Auftrag Jesu wird lediglich in der Rechtfertigung der Jünger innerhalb der Erzählung ausgedrückt 
$\pi \rho о \sigma-$ ist kaum ergiebiger: Sie ist auf wenige Vorkommnisse in den Synoptikern und in der Apostelgeschichte beschränkt. ${ }^{399}$ Ergiebiger, was paulinische Belege angeht, scheint auf den ersten Blick tó $\sigma \sigma \omega$ ohne präpositionales Vorderglied zu sein. Denn neben zwei synoptischen Belegen ${ }^{400}$ und vierfacher Verwendung in der Apostelgeschichte ${ }^{401}$ erscheint das Verb auch zwei Mal bei Paulus, in Röm 13,1 und 1. Kor 16,15. Allerdings ist dort - wie auch bei den anderen Belegen mit Ausnahme von Mt 28,16 - nicht von einer Anordnung die Rede, sondern eine

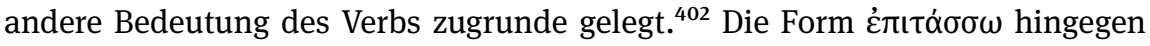
erscheint einerseits einige Male bei Markus und ein einziges Mal in der Apostelgeschichte ${ }^{403}$ - darüber hinaus aber auch an einer Stelle im corpus Paulinum, nämlich in Phlm 8.

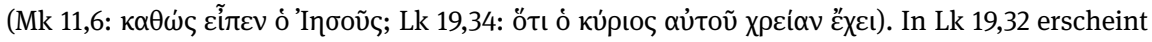

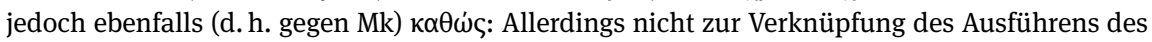
Befehls mit dem Auftrag, sondern zur Verbindung des Vorfindens des Angekündigten. Anders ist die Situation beim Beleg in Mt 26,19, wo Lk 22,13 mit (dem etwas ausführlicheren) Mk 14,16 das Motiv des Vorfindens des Angekündigten gemein hat, im matthäischen Text aber die Ausführung durch die Jünger mit dem Auftrag durch Jesus verbunden wird. Mt 27,10 schließlich ist Teil des Mischzitates in der Perikope zum Ende des Judas.

399 Mt 1,24 bezieht sich auf das Befehlen des Engels an Josef. Mt 1,24/Mk 1,44/Lk 5,14 haben alle dieselbe Form des Verbs, auch wenn es jeweils unterschiedlich (geschickt) eingebunden ist. In der Apostelgeschichte erscheint es im Mund des Paulus in Apg 17,26. Interessant ist zudem der Gebrauch in 10,48 - der einzigen Stelle im NT, wo tatsächlich ein Infinitiv von diesem Verb

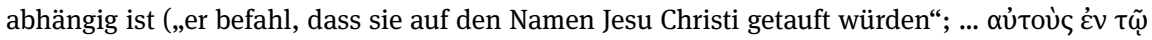

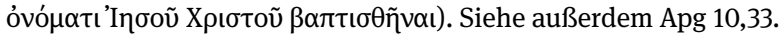

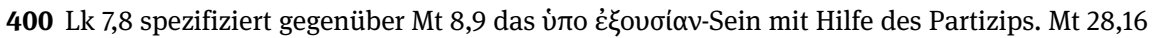
eröffnet den Abschnitt zum Missionsbefehl, wobei das Verb hier durch den Dativ der Empfänger der Anordnung ergänzt ist.

401 Apg 13,48, 15,2, 22,10, 28,23.

402 Wenn man auch Apg 22,10 dieser Bedeutung zuordnen würde, so wäre hier immerhin vom Paulus „Befohlenen“ die Rede. Vgl. LUT: „Steh auf und geh nach Damaskus. Dort wird man dir sagen, was dir zu tun aufgetragen ist.“ Das, was für Paulus in Damaskus zu tun ist (vgl. Apg 9,6: ...

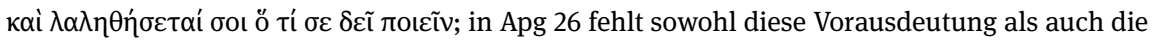
dann erzählte Handlung), sind für ihn „vorgesehene“ Handlungen, die ihm aber eben gerade noch nicht ,aufgetragen“ sind, sodass sie ihm erst „gesagt werden“ müssen ( $\lambda \alpha \lambda \eta \theta \dot{\sigma} \sigma \varepsilon \tau \alpha \mathrm{l})$.

403 Mk 1,27 // Lk 4,36 zum Befehligen der unreinen Geister (keine Entsprechung der Perikope bei Matthäus). Die Schilderung des Todes von Johannes des Täufers ist hingegen in der langen

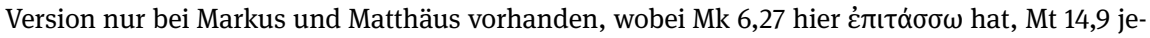

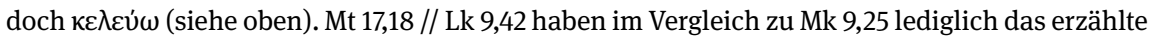

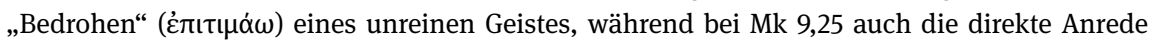

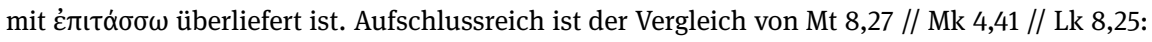
Auf die Sturmstillung folgt bei allen das Erstaunen darüber, dass Wind und Wasser Jesus gehorchen (ن் $\alpha \kappa о v ́ \omega)$, nur Lukas fügt jedoch den zuvor ergehenden Befehl ein (und ein konsekutives

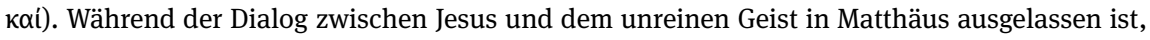




\subsubsection{4 ,Command, order': દ̇mıtó $\sigma \sigma \omega$ in Phlm 8}

Diese Stelle ist nun nicht dadurch signifikant für die hier verfolgte Fragestellung, dass hier durch einen Befehl ein - zukünftiges Geschehen einschließendes - Protonarrativ kreiert würde, sondern weil Paulus hier explizit auf das Befehlen zur Durchsetzung seines Wunsches verzichtet und zudem dieses Vorgehen auch noch reflektiert.

Zunächst versichert Paulus in Phlm 8, er hätte durchaus große Freimütigkeit

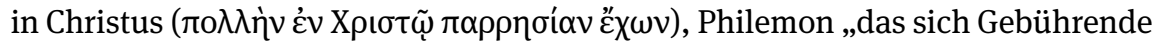

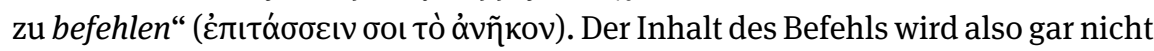
durch einen Infinitiv oder abhängigen Begehrsatz spezifiziert, sondern durch das unpersönlich gebrauchten Partizip zur „Selbstverständlichkeit,“404 die gar keine explizite Erwähnung bedarf. ${ }^{405}$

Paulus entwirft die Beziehung zu Philemon somit als ein Verhältnis, in welchem der Apostel die Autorität hat, bindende Anweisungen zu erteilen. ${ }^{406}$ Obwohl dies so sei ${ }^{407}$ möchte Paulus allerdings, wie V. 9, formuliert, „um der

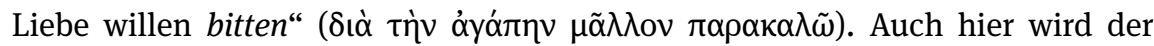
Inhalt jedoch bezeichnenderweise nicht erwähnt, da das Verb absolut gebraucht wird, um den Kontrast zum Modus des Befehlens aus V. 8 zu betonen. Stattdessen liefert der Vers eine ausführliche Beschreibung des Bittstellers. ${ }^{408}$

V. 10 setzt dann erneut mit $\pi \alpha \rho \alpha \kappa \alpha \lambda \varepsilon \dot{\varepsilon} \omega$ ein - und spezifiziert diesmal denje-

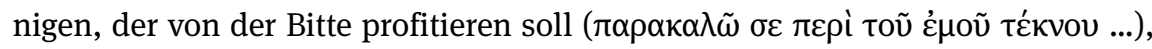
schiebt die Nennung des Erbetenen also immer noch auf. Erst in V. 13 wird dann der Wunsch zum ersten Mal angedeutet, indem auf einen vergangenen Wunsch Bezug genommen wird (siehe hierzu Abschnitt 4.4.2.2). ${ }^{409}$

Die Unterscheidung zwischen $\dot{\pi} \pi ı \alpha \dot{\sigma} \sigma \sigma \omega$ und $\pi \alpha \rho \alpha \kappa \alpha \lambda \dot{\varepsilon} \omega$ wird im Anschluss in V. 14 erneut aufgegriffen, indem nun die jeweils resultierenden Handlungen

hat Mk 5,10 die Bitte, Jesus möge ihn nicht aus der Gegend vertreiben, während Lk 8,31 dieses

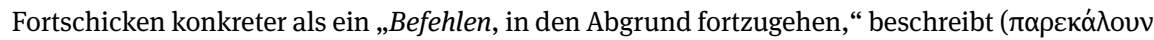

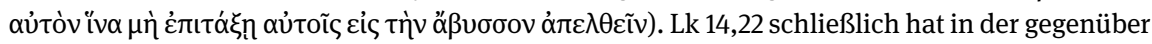
Mt 22,9-10 ausführlicheren, da doppelten Einladung von zusätzlichen Hochzeitsgästen einen Bericht des Dieners an den Herrn über die Ausführung des Befohlenen. In Apg 23,2 ist Paulus

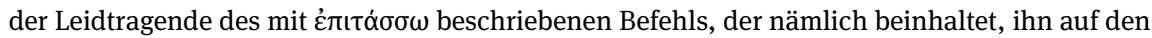

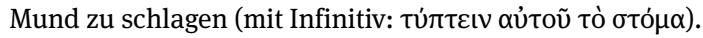

404 Vgl. auch V. 19 zur „Schuld“ des Philemon gegenüber Paulus.

405 Vgl. auch die Belege aus den Papyri bei Arzt-Grabner, Philemon, 200.

406 Vgl. Arzt-Grabner, Philemon, 66 und 199-200 für Belege aus Weberlehrverträgen.

407 Die Sinnrichtung des Partizips ist konzessiv aufzufassen.

408 Vgl. Arzt-Grabner, Philemon, 201 zur Funktion in antiken Briefen.

409 Siehe dazu unten, 5.3.2.4. 
angesprochen werden: Philemons „Wohltat“ (

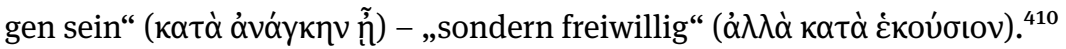

Der eigentliche Inhalt der Bitte wird dann erst in V. 17 spezifiziert - interessanterweise durch einen expliziten Imperativ (,nimm ihn auf wie mich!“; $\pi \rho \circ \sigma \lambda \alpha \beta o \tilde{~}$

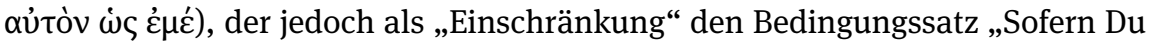

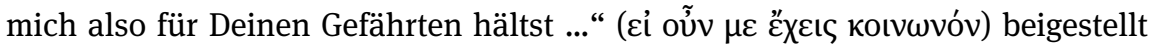
bekommt. Durch diesen wird auch der Form nach der Umstand gewahrt, dass

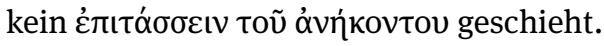

Das Protonarrativ der unterlassenen aber theoretisch möglichen verbindlichen Anordnung dient hier dazu, die freiwillige Befolgung der Bitte (V. 10.14) sicherzustellen: Es wäre ein Handlungszusammenhang möglich, der zum selben Ziel führen würde, für Philemon jedoch weniger angenehm wäre. Es liegt daher auf der Hand, dass er stattdessen den Weg wählen sollte, der für ihn nicht mit einem Gesichtsverlust einhergeht.

Ob die hier zur Wahl gestellten Protonarrative tatsächlich in der Handlung vergleichbar sind, kann natürlich hinterfragt werden: Könnte eine befohlene

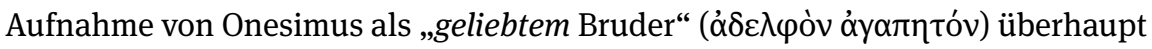
gelingen, hat Paulus diese alternative Option also überhaupt oder ist er nicht letztlich auf Philemons nicht erzwingbare Einsicht angewiesen? Und selbst wenn durch den unverblühmten Befehl derselbe Endzustand erreicht werden könnte, gäbe es nicht doch einen Unterschied für die Beziehung zwischen Paulus und Philemon, wenn die Umsetzung des paulinischen Willens nicht „in Liebe“ (V. 9) geschehen würde? Diese Schwierigkeiten zu überkommen, wäre aus der Sicht des Paulus freilich eben Philemons Aufgabe, da das im kontrafaktischen Hand-

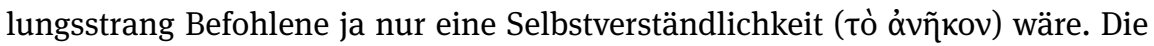
eventuell fehlende Schlüssigkeit eines solchen Narrativs hat also durchaus auch eine gelegen kommende rhetorische Funktion im konkreten Kontext: Zur Unannehmlichkeit des einen Handlungsstrangs kommt nämlich so noch die Schwierigkeit seiner Umsetzung hinzu. Auch wenn Paulus also nicht befiehlt, bewirkt das explizite Diserzählen des Befehls gerade eine Steigerung der Wahrscheinlichkeit des Eintretens der gewünschten Ereignisse.

Auch wo Paulus eine solche explizite Reflexion nicht vornimmt, dürfte nicht selten ein Kontrast zwischen der ihm möglichen und der tatsächlich gewählten Ausdrucksweise einen die Umsetzung motivierenden Faktor darstellen. Nicht immer freilich - besonders nicht im Römerbrief hat er dabei so viel Autorität auf seiner Seite wie im Fall seiner Beziehung zu Philemon (V. 19). Dementsprechend dürfte die Formulierung des Anliegens als Bitte auch unterschiedlich stark das Protonarrativ eines autoritativeren Alternativvorgehens mitschwingen lassen.

410 Zum Gebrauch des Adjektivs vgl. GGNT 137c. 
$\mathrm{Zu}$ beachten ist schließlich: Die (Nicht-)Anordnung in V. 17 leuchtet nicht nur vor dem Hintergrund des diserzählten Befehlens auf, sie erscheint auch inhaltlich als recht „kleine“ Bitte, weil im Hintergrund seit V. 13 ein ganz anderes Verlangen mitläuft: der Wunsch des Paulus, Onesimus für sich zu behalten.

Wie unten (bei den finalen Konnexionen, Abschnitt 5.3.2.4) gezeigt werden soll, wird durch V. 14 der Akt des freiwilligen Übergebens des Sklaven an Paulus nicht aufgegeben. Die Zuversicht (das „Wissen“!), ${ }^{411}$ dass Philemon mehr tun werde, als das, was Paulus „sagt“ (d.h.: wozu er in V. 17 explizit auffordert), wird dieses Anliegen implizit wieder aufnehmen. Für die Aufforderung in V. 17 steht jedoch erst einmal im Hintergrund, dass Paulus gerade nicht um diese größere - Sache explizit ersucht, wodurch die explizite Bitte zusätzlich gestützt wird.

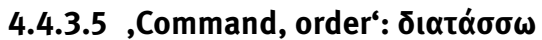

$\mathrm{Zu}$ betrachten ist nun nur noch die Verbindung von $\tau \alpha$ ó $\sigma \omega \omega$ mit $\delta \iota \alpha-$, welche matthäisch ${ }^{412}$ und lukanisch (auch 5 Mal in der Apostelgeschichte) bezeugt ist, darüber hinaus aber auch 6 Mal im corpus Paulinum. Bis auf Lk 3,13 ist hier auch in allen Fällen ${ }^{413}$ das anordnende Element in der Semantik enthalten.

In Apg 23,31 und 24,23 $3^{414}$ wird mit dem Verb spezifisch auf Befehle in einem militärisch/politischen Kontext Bezug genommen. Auch das Edikt des Claudius in Apg 18,2 wird mit diesem Verb umschreiben: „... weil Claudius befohlen hatte,

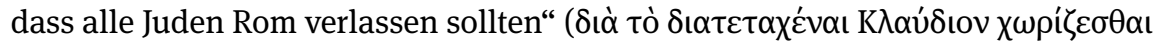

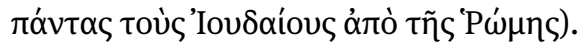

In den Paulusbriefen wird das Wort ohne abhängige Infinitiv- oder Nebensatz-Konstruktion für die Anordnungen gegenüber dem Mitarbeiter Titus

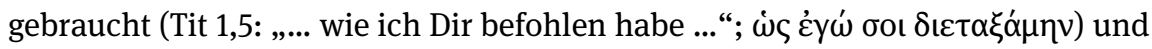
für die Anordnung des Gesetzes „durch Engel, durch die Hand eines Mittlers“

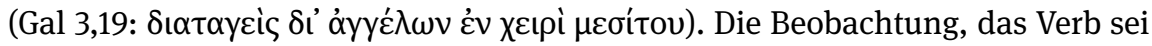

411 Siehe hierzu bei den abhängigen Behauptungssätzen zu Phlm 21, Kapitel 13, Abschnitt 3.1. 412 Mt 11,1 - der einzige matthäische Beleg - ist ohne Parallele. Die Ergänzung der Abschlussformulierung durch das „Befehlen“ geht über die sonst übliche Formulierung bei Matthäus hinaus. Lk 3,13 und 17,9.10 gehört zum lukanischen Sondergut. Anders Lk 8,55: Der Befehl, man

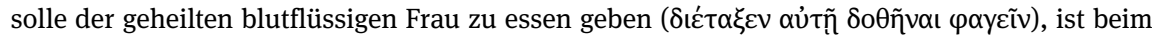
(stark kürzenden) Mt (9,18-26) und auch beim insgesamt in dieser Perikope ausführlicheren Mk (5,21-43) nicht enthalten. Für die Apostelgeschichte siehe Apg 7,44, 18,2, 20,13, 23,31, 24,23.

413 Diskutiert werden könnte lediglich noch die Anordnung/das „Arrangement“ in Apg 20,13. 414 Hier mit Infinitiven des Befohlenen. Die Konstruktion mit passivischem Infinitiv und Akku-

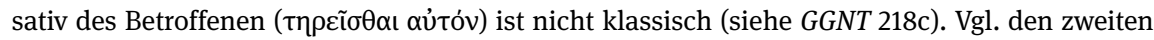
Infinitiv des AcI (čxદiv). 
„never used elsewhere in Biblical Greek to refer to the giving of the law,“ muss nicht bedeuten, dass es in Gal 3,19 ein „unusual verb“ sei. ${ }^{415}$ Es ist in der griechischen Literatur - auch bei Philon und Josephus ${ }^{416}$ - vielmehr eine gängige Wahl sowohl für den Erlass von Gesetzen als auch für das darin Gebotene. ${ }^{417}$

Tatsächlich „ungewöhnlich“ ist hingegen angesichts der in der bisherigen Untersuchung sich als so zurückhaltend eingesetzt zeigenden Terminologie des

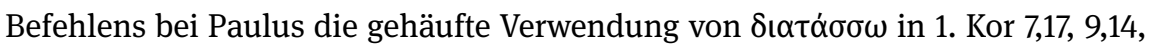
$11,34,16,1$.

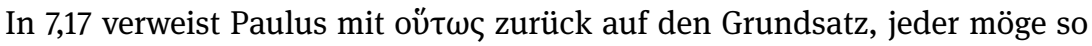
wandeln, wie der Herr es einem zugeteilt bzw. wie Gott einen berufen habe (Ei $\mu$ ì

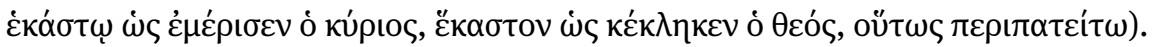

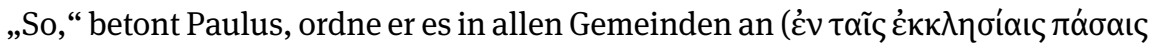
$\delta ı \alpha \tau \alpha ́ \sigma \sigma o \mu \alpha \iota)$. Auch in 16,1 liegt ein Vergleich vor: Bezüglich der Sammlung für die

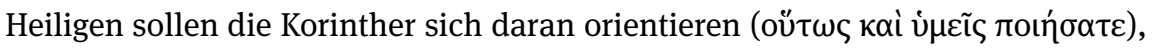

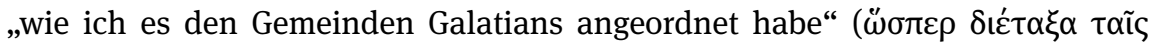

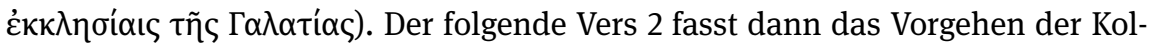
lekte zusammen. Woher die Korinther genau von der Beteiligung der Galater und von den an sie gerichteten Instruktionen des Paulus wissen, bleibt unklar. Auf jeden Fall wird aber deutlich, dass Paulus den Verweis auf die vergangene und vermutlich erfolgreiche ${ }^{418}$ Anordnung gezielt einsetzt, um seiner Aufforderung zum Handeln Gewicht zu verleihen. Neben dem überzeitlichen Anordnen in 7,17 und dem auf die Vergangenheit verweisenden Befehl in 16,1 liegt in 11,34 auch ein Verweis auf die Zukunft vor: „Wenn ich ankomme“ ( $\dot{\omega} \varsigma$ âv है $\lambda \theta \omega)$, „werde ich das

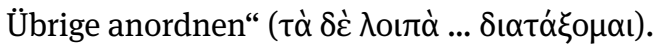

Ein von $\delta \_\alpha \tau \alpha ́ \sigma \sigma \omega$ abhängiges Verb findet sich nur in 1. Kor 9,14, wo Paulus auf ein Herrenwort verweist: „Der Herr hat den Verkündigern des Evangeliums

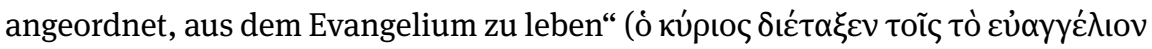

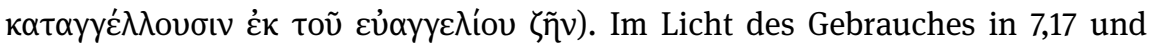
16,1 (vgl. Tit 1,5) macht es wenig Sinn, den Dativ abgeschwächt als einen dativus commodi aufzufassen, wodurch die Verkündigenden auch grammatisch zu Nutznießern der Anordnung würden.

Instruktiv ist der Vergleich der Sendungsanweisung, wie sie bei Mt überliefert

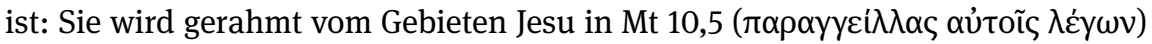

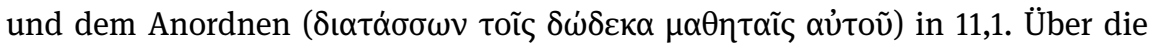

415 Moo, Galatians, 235.

416 Vgl. etwa Philon, Spec. 2.79 und Josephus, A. J. 4.249.

417 Vgl. etwa Hesiod, Op. 277 und Diodor, Bib. hist. 13.34.6.

418 Schrage, Brief IV, 427 ist skeptisch, was eine Vorildfunktion der Galater angeht. Da die genauen Hintergründe unbekannt sind, muss die genaue Stoßrichtung letztlich offen bleiben. 
Aufforderung zum Verzicht auf spezielle Vorkehrungen zur Deckung der Grundbedürfnisse in 10,10 wird auch die begründende Versicherung „Denn der Arbei-

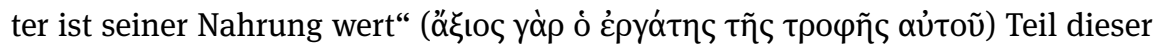
„Anordnung.“

Die auffällige Wortwahl bei Paulus in 1. Kor 9,14 wird daher auch am ehesten vor dem Hintergrund der Formulierung in der ihm vorliegenden Tradition zu erklären sein. So wird verständlich, wie er im Folgevers 9,15 - unter anderem auf dieses Gebot des Herrn zurückgreifend sagen kann, dass er davon keinen

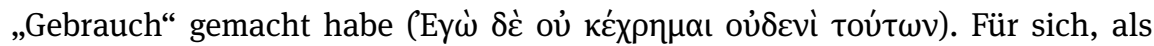
einen der Verkündigenden, übernimmt Paulus also wohl vor allem den Aspekt der absoluten Unhinterfragbarkeit des Rechts auf Versorgung im Dienst.

Man braucht folglich nicht anzunehmen, dass in Paulus' Darstellung sein eigenes Vorgehen gegen die Anordnung des Herrn verstoße ${ }^{419}$ oder auch, etwas abgeschwächt, zumindest über „ein verpflichtendes und nicht in die Beliebigkeit abzuschiebendes Gebot des Herrn“ hinausgehe. ${ }^{420}$ Wohl aber ist es zutreffend,

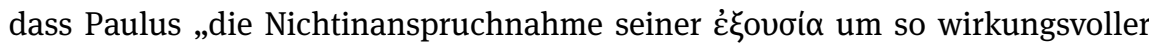
kontrastieren kann, “421 als dass es sich von dem Protonarrativ abhebt, das durch den Verweis auf den Herrn und seine autoritative Äußerung den eigentlich zu erwartende Handlungsstrang absteckt.

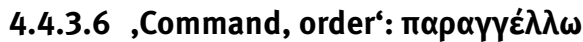

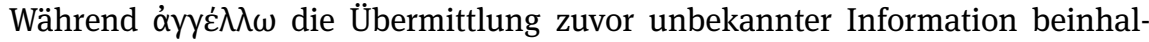
tet $^{422}$ und im Neuen Testament lediglich in Joh 20,18 erscheint, sind die Formen mit $\alpha$ ró und $\pi \alpha \rho \alpha ́$ semantisch für die hier verfolgte Frage bedeutsamer und auch häufiger.

Was $\alpha \dot{\pi} \alpha y=\varepsilon \dot{\lambda} \lambda \omega$ angeht, erscheint es zwar im NT $45 \mathrm{Mal}$ - darunter auch zwei Mal in den Paulusbriefen (1. Kor 14,25 und 1. Thess 1,9), doch ist in nahezu allen Fällen deutlich, dass die Bedeutung des Berichterstattens gemeint ist. ${ }^{423}$ In der Tat ist zweifelhaft, ob die semantische Bandbreite überhaupt über diesen Bereich hinaus ausgedehnt verstanden werden sollte. ${ }^{424}$

419 Die Annahme von Klauck, Herrenmahl, 66, Paulus greife den Vorwurf auf, er habe gegen ein Gebot Jesu selbst verstoßen, überzeugt daher im Kontext nicht.

420 Schrage, Brief II, 310.

421 Schrage, Brief II, 310.

422 LN 33.189: ,to provide otherwise unknown information.

423 Vgl. LN 33.198: ,to announce or inform, with possible focus upon the source of information. Vgl. dementsprechend den abhängigen ötı-Satz in 1. Kor 14,25.

424 Vgl. etwa LSJ 173. Bei BA 157/158 BDAG 787 ist als separate Bedeutung das öffentliche Bekanntmachen einer Sache, die auch in der Gegenwart oder Zukunft angesiedelt sein kann, ab- 
Das Verb $\pi \alpha \rho \alpha y y \varepsilon ́ \lambda \lambda \omega$ ist hingegen ganz auf die Übermittlung von Befehlen beschränkt. ${ }^{425}$ Während die synoptischen Belege vergleichsweise spärlich sind, ${ }^{426}$ erscheint das Verb immerhin $11 \mathrm{Mal}$ in der Apostelgeschichte und $12 \mathrm{Mal}$ im corpus Paulinum.

Die drei Vorkommnisse in den unumstrittenen Briefen sind 1. Kor 7,10, 11,17 und 1. Thess 4,11. Im letztgenannten Vers sind die Infinitive, welche ehrbare Arbeit umschreiben, nicht direkt abhängig von $\pi \alpha \rho \alpha y y \varepsilon ́ \lambda \lambda \omega$ sondern von der Bitte in V. 10: „Wir bitten euch aber, Brüder, mehr und mehr zuzunehmen und eure

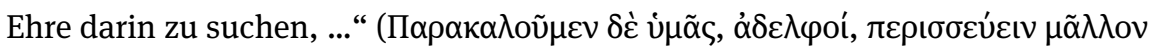

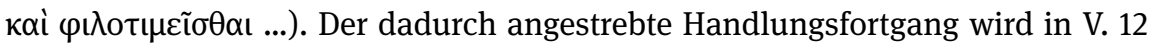
durch einen finalen îvo-Satz expliziert. Zuvor jedoch wird durch eine kurze Rückblende das gegenwärtige Bitten (vgl. oben, Abschnitt 4.4.3.1) mit einem bereits in der Vergangenheit ergangenen Gebieten in Verbindung gebracht: „... - wie

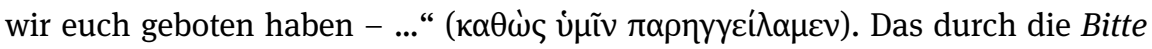
eröffnete Protonarrativ wird damit in Kontinuität mit der anfänglichen Unterweisung gestellt. Die terminologische Unterscheidung von $\pi \alpha \rho \alpha \kappa \alpha \lambda \varepsilon \dot{\varepsilon} \omega / \pi \alpha \rho \alpha \gamma \gamma \varepsilon \dot{\varepsilon} \lambda \lambda \omega$

gegrenzt. Die Konstruktion mit ïva wird dann als eine spezielle Verwendungsweise verstanden: „to introduce a command that is to be transmitted to another.“ Als einziges Beispiel im NT wird Mt 28,10 angeführt. Vgl. aber die Ballung des Verbs in dieser Passage (V. 8 und V. 11 für das Berichten) und die Anmerkungen von Robertson, Grammar, 993 zur Nähe von ïva zu ö $\tau$ in solchen Fällen. Eine allgemeinere Bedeutung - vergleichbar zum deutschen ,ausrichten“ - sollte daher

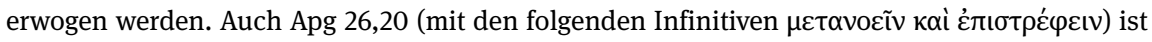
indirekte Rede. Ebenso in der v. 1. in Apg 17,30.

425 Vgl. auch BDAG 5560: ,to make an announcement about someth. that must be done.

426 Mt 10,5 ist in der Aussendung der Jünger ohne Parallele. Mt 15,35 fügt wie Mk 8,6 an $\pi \alpha \rho \alpha y \gamma \varepsilon \dot{\lambda} \lambda \omega$ (dort jedoch als finites Verb im historischen Präsens) den Infinitiv der Aufforderung an. Interessant ist Mk 6,8 // Mt 10,9 // Lk 9,3: Bei Markus folgt ein abhängiger ǐva-Satz, der im

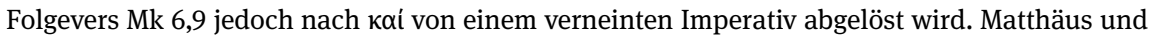
Lukas gehen im Detail unterschiedliche Wege, lösen aber beide auch diesen Teil als direkte Rede auf. Im Zusatz von Mk 16,8 wird das passive Perfekt-Partizip substantiviert verwendet, um auf die „Instruktionen“ zurückzuverweisen. Lukas lässt das „,bedrohliche“ ‘ $\mu \beta \rho \mu \alpha \dot{\alpha} о \mu \alpha$ เ aus Mk 1,43.

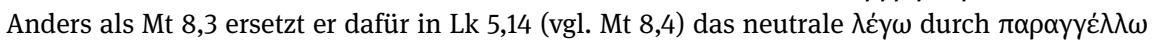
(und vermeidet so auch doppeltes $\lambda \varepsilon \dot{\gamma} \omega, \mathrm{d}$. h. sowohl als Redeeinleitung als auch als Inhalt der Rede). Eine ähnliche Spezifizierung findet gegenüber dem „Reden“ zum unreinen Geist in Mk

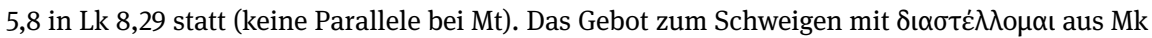

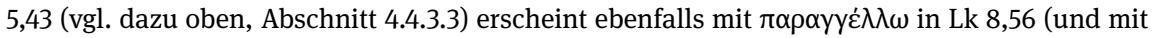

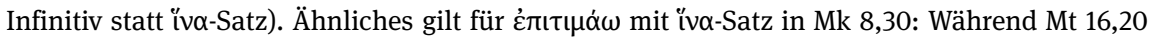

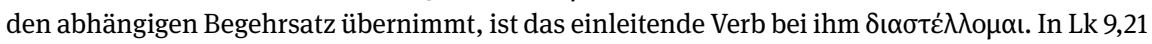

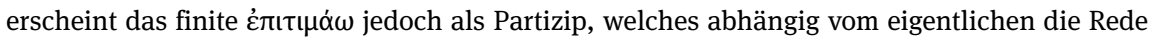
(mit Infinitiv) einleitenden Verb $\pi \alpha \rho \alpha y=\dot{\varepsilon} \lambda \lambda \omega$ ist. Nimmt man zudem die Belege in der Apostelgeschichte hinzu $(1,4,4,18,5,28,5,40,10,42,15,5,16,18,16,23,17,30,23,22)$, so wird man in diesem Fall tatsächlich berechtigter Weise von einem lukanischen „Vorzugswort“ sprechen dürfen. 
signalisiert dabei den in der Zwischenzeit bereits erzielten Fortschritt, sodass nun „nur noch“ sanfte Motivation vonnöten ist.

Ein Bezug auf die Vergangenheit liegt letztlich auch in 1. Kor 7,10 vor, wo mit dem Verb ein an die Verheirateten gerichtetes Gebot eingeleitet wird: „dass

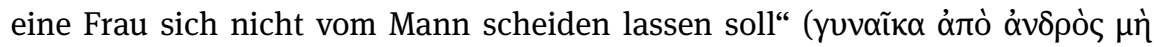

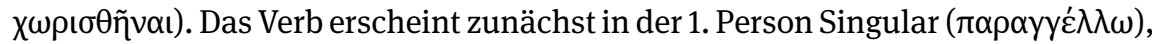
doch das Subjekt wird zugleich „korrigiert“: „Ich befehle - nicht ich, sondern der

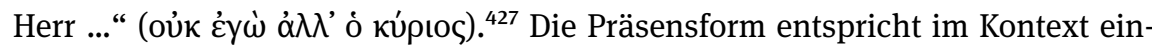
deutig den anderen Anweisungen mit $\lambda \varepsilon ́ \gamma \omega$ in 7,8 und 7,12 (hier mit „bestätigtem“ Subjekt: ćyc̀ oủx ó kúpıoৎ) und sollte nicht mit der dauernden „Geltung“ des Herrenwortes in Verbindung gebracht werden. ${ }^{428}$

Während Paulus in 1. Kor 11,2 Lob für die Korinther übrig hat, merkt er in 11,17 an, dass ihm dies angesichts der folgenden Ermahnungen nicht möglich sei

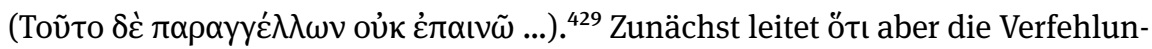
gen aus Sicht des Paulus ein, die bis V. 21 expliziert werden. Auf diese Erzählung folgend, skizziert V. 22 durch das Mittel der Fragesätze einen alternativen Handlungsstrang („Habt ihr denn nicht Häuser, um zu essen und zu trinken?“; $\mu$ 门̀ yò $\rho$

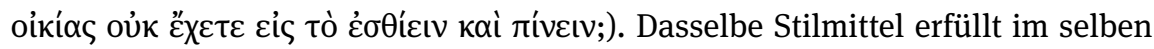
Vers noch zwei weitere Funktionen: Da die Korinther natürlich diese erste Frage nicht verneinen können, wirft Paulus die Frage auf, ob in der vorangegangenen Erzählung eventuell ein Element fehle, das ihre Motivation betreffen würde: „Oder verachtet ihr die Gemeinde Gottes und beschämt die, welche nichts haben?“

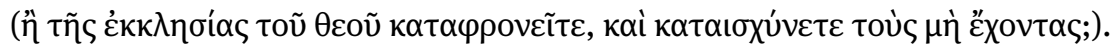

Paulus unterstellt derart Gravierendes nicht direkt, doch signalisiert die durch die abschließenden zwei Fragen zum Ausdruck kommende Ratlosigkeit

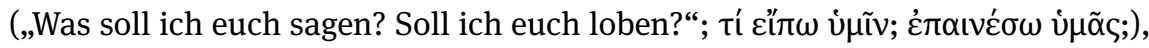
dass in seinen Augen das korinthische Verhalten kaum in einer kohärenten Erzählung wiedergegeben werden kann, die zugleich nicht schwerwiegendes Fehlverhalten bzw. eine derart verabscheuungswürdige Einstellung der Korinther beinhalten würde.

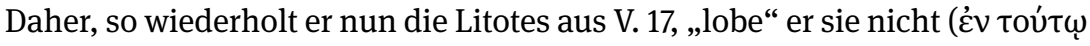

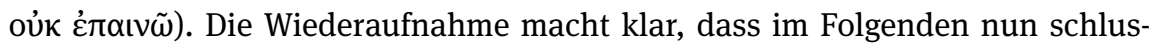
sendlich das angekündigte Befehlen eines alternativen, besseren Verhaltens erwartet werden darf. Eine eigentliche Aufforderung - die zur individuellen

427 Siehe dazu oben, Kapitel 12, Abschnitt 4.

428 Gegen Schrage, Brief II, 97. Siehe auch dessen leichte Einschränkung in Fußnote 256 auf S. 98.

429 Die ungewöhnliche Konstruktion mit vorausweisendem Demonstrativpronomen und vorwegnehmendem Verweis auf den Befehl hat zu zahlreichen Varianten geführt. 
Selbstprüfung - erfolgt allerdings erst in V. 28. Zuvor (V. 23-25) liefert Paulus in einer Rückblende eine Erzählung über die Einsetzung des Abendmahls durch Jesus sowie in V. 26-27 eine Interpretation der christlichen Abendmahlspraxis vor diesem Hintergrund.

Diese explizite Erzählung fungiert hier also nicht direkt „als Korrektiv und Norm, “430 stützt aber die Aufforderung in V. 28, indem sie deren Notwendigkeit herausarbeitet. Entsprechend verfolgt V. 29 mit Hilfe eines konditionalen Partizips ${ }^{431}$ das Protonarrativ, welches aus dem Nichtbefolgen dieses Grundsatzes

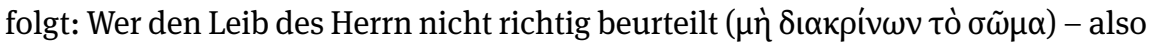
die in V. 26-27 erläuterte Tragweite außer Acht lässt und entsprechend die selbstkritische Prüfung der eigenen Person in V. 28 unterlässt - für den ist der an sich „profane“ Akt des Essens und Trinkens ein zum Gericht führendes Handeln („Der

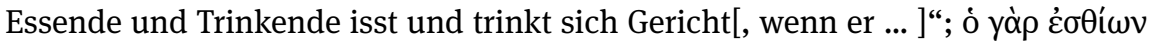

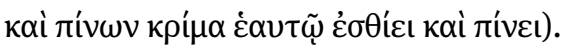

Vor diesem Hintergrund betrachtet, ist die im folgenden Vers 30 getroffene Aussage - „Deshalb sind viele unter euch schwach und krank und nicht

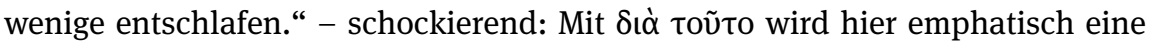
Grund-FOLGE-Konnexion hergestellt. ${ }^{432}$ In dieser Analyse der gegenwärtigen Situation der korinthischen Christen werden die entsprechenden negativen Erlebnisse kausal darauf zurückgeführt, dass das Protonarrativ in V. 29 eben nicht nur rein hypothetisch ist. Damit wird letztlich auch die verheerende, noch in V. 22 nur in Form einer Frage angedeutete, Analyse der bisherigen Handlung rückwirkend bestätigt.

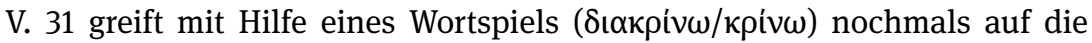
Aufforderung zur Selbstprüfung aus V. 28 zurück. Allerdings trägt die irreale Konditionalperiode (vgl. zur Stelle entsprechend oben, Kapitel 12, Abschnitt 8.3) eben der Tatsache Rechnung, dass dieser Grundsatz bisher nicht ausreichend befolgt wurde und sich das Protonarrativ des erfolgenden Gerichts letztlich als die tatsächliche Handlung herausgestellt hat. Es ist daher nun ironischerweise das Nichtgerichtetwerden, welches als hypothetisches Ereignis in einem alternativen Handlungsstrang erscheint. Entsprechend ist in V. 32 auch die im Partizip крıvó $\mu \varepsilon v o$ enthaltende Bedingung als erfüllt zu betrachten: „Wir“ erfahren gegenwärtig tatsächlich Gericht, denn das Protonarrativ von V. 28 - mitsamt dem dort genannten крí $\mu \alpha$ - entspricht den Ereignissen in Korinth.

430 Schrage, Brief III, 29.

431 Vgl. allgemein zur konditionalen Konnexion unten, Abschnitt 5.2.

432 Vgl. GGNT 333a. 
Damit könnte nun freilich die Erzählung, ganz ohne glücklichen Abschluss, zu Ende gehen. Paulus hingegen geht anders vor: Zwar hat sich das Protonarrativ der unwürdigen Korinther tatsächlich als wahr herausgestellt. Die heilvolle Alternativhandlung zu dieser Unheilsgeschichte lässt sich jedoch zumindest noch für die Zukunft erreichen. Ja, letztlich ist auch die göttliche Strafe als ein die Handlung nicht zum jähen Ende bringendes, sondern vorantreibendes Moment zu ver-

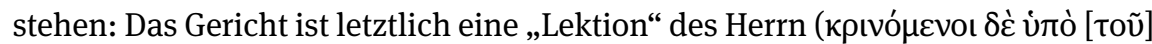
кupíov $\pi \alpha ı \varepsilon \varepsilon v o ́ \mu \varepsilon \theta \alpha$ ), welche mit Blick auf die Zukunft eben gerade den Zweck hat, die Christen auf einen Weg zu bringen, der nicht zur Strafe im Endgericht

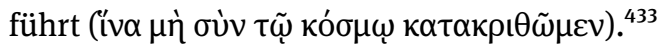

Wie schon in V. 27 wird daraufhin in V. 33 mit $\ddot{\omega} \sigma \tau \varepsilon$ ein selbstständiger Satz eingeleitet, welcher eine Schlussfolgerung aus dem zuvor Gesagten zieht: Jetzt erfolgt schlussendlich das angekündigte $\pi \alpha \rho \alpha \gamma \gamma \varepsilon \dot{\lambda} \lambda \varepsilon \iota v$ in Weiterführung von V. 28, begründet nicht nur durch eine vage Unzufriedenheit, sondern durch die Einordnung in einen komplexen narrativen Bezugsrahmen.

Die Vorkommnisse von $\pi \alpha \rho \alpha y \gamma \varepsilon \dot{\lambda} \omega \omega$ im Zweiten Thessalonicherbrief sind insbesondere durch ihre Häufung in Kapitel 3 auffällig. Das erste Mal begegnet das Verb in 2. Thess 3,4. Nach der Versicherung der Treue des Herrn in 3,3 wird nun der Verweis auf ein Gebieten von manchen Exegeten als diskontinuierlich empfunden. ${ }^{434}$ Dessen Beistand aber ist wohl gerade vorausgesetzt, wenn das in 3,4 geäußerte Vertrauen als „im Herrn“ geschehend qualifiziert wird ( $\pi \varepsilon \pi \operatorname{li}^{\prime} \theta \alpha \mu \varepsilon v$

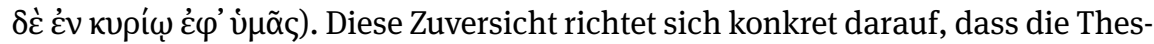

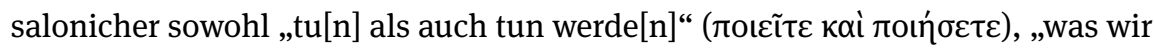

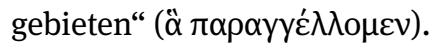

Auf diesen überzeitlichen Bezug auf die paulinische Instruktion folgt zunächst in V. 5 eine an den Herrn gerichtete Aufforderung, welche die Ausrichtung der Herzen der Thessalonicher betrifft. In V. 6 begegnet dann wieder $\pi \alpha \rho \alpha y \gamma \varepsilon \dot{\varepsilon} \lambda \omega \omega$ im Präsens, wobei diesmal ein direkter Bezug auf eine im Text folgende Anordnung vorliegt, nämlich „dass ihr euch zurückzieht von jedem Bruder, der unordentlich wandelt und nicht nach der Überlieferung, welche ihr von uns empfangen habt“

\footnotetext{
433 Auch hier ist freilich die Möglichkeit einer solchen Handlungsfortsetzung implizit gegeben, denn Paulus spricht eben nicht nur von der generellen Möglichkeit einer eschatologischen Verurteilung, sondern verweist vielmehr auf einen tatsächlich bereits existierenden parallelen Handlungsstrang.

434 Trilling, Brief, 137 spricht von einem „Sprung,“ den er auch nicht dadurch eliminiert sehen will, dass man „einen Zwischengedanken zugrunde legt ...: Der Herr wird das Seine in Treue tun - aber die Gemeinde muß auch das Ihre tun.“ Dieser Abweisung ist zuzustimmen, auch wenn die von Trilling behauptete Verschiebung des Fokus auf den „Apostel selbst“ ebenfalls kaum dem Text gerecht wird.
} 


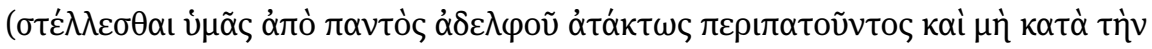

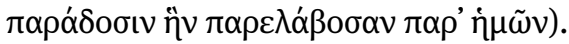

Diese $\pi \alpha \rho \alpha ́ \delta$ oбıৎ ist natürlich nicht nur dem Wortsinn nach eine Überlieferung, sondern dem Wesen nach spezieller eine Instruktion - wie sie in 1 . Thess 4,11 ja auch bereits mit $\pi \alpha \rho \alpha y \gamma \varepsilon \dot{\varepsilon} \lambda \omega$ zum Ausdruck gebracht wurde. Ebenso wird

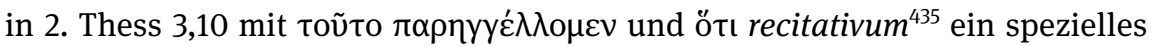
Gebot zitiert: „Wenn jemand nicht arbeiten will, so soll er auch nicht essen“ (عl

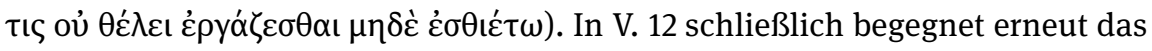

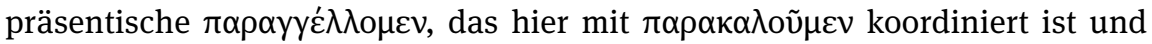
von einem ǐvo-Satz gefolgt wird, der das positive Gegenstück dieses Protonarrativs ausdrückt und für die Gegenwart aktualisiert: „... ruhig arbeitend, das eigene Brot $z u$ essen.“

Dass der ganze Abschnitt auf eine Verhaltensänderung abzielt, zeigt schon die Tatsache, dass hier eben nicht - der Apodosis in V. 10 entsprechend - die Aufforderung ergeht, einfach „nicht zu essen.“ Das vielmehr an „die solchen“

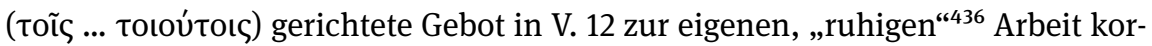

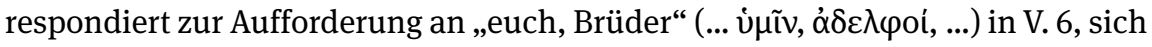
von jedem auf besagte Weise falsch verhaltenden Bruder abzusondern. Spezifischer dient die erste Handlung als Instrument dazu, den in V. 12 Angesprochenen keine andere Wahl zu lassen, als ebenfalls dem an sie ergehenden Gebot nachzukommen. ${ }^{437}$

Diese/s abschließende Bitte/Gebot ist, wie wir oben (Kapitel 11, Abschnitt 2; Kapitel 12, Abschnitt 2.3.2) bereits gesehen haben, freilich nicht nur durch diese lexikalischen Querverbindungen $\mathrm{zu}$ früherem Gebieten und dem generell gültigem Befolgen eben dessen (V. 4) gestützt, sondern auch durch ein komplexes Netzwerk narrativer Verknüpfungen. ${ }^{438}$

$435 \mathrm{Zu}$ einer alternativen Auflösung der Syntax siehe oben, Kapitel 7, Abschnitt 4.2.2.

436 Vgl. 1. Thess 4,11, wobei dort an die Außenwirkung gedacht ist (vgl. auch 1. Tim 2,2), während hier (vgl. etwa 3,8) wohl eher an einen zu vermeidenden Ausgleich innerhalb der Gemeinde gedacht ist, welcher durch die Untätigkeit mancher notwendig wird.

437 Unerklärlich ist daher die Einschätzung von Marxsen, Thessalonicherbrief, 101, der Verfasser könne in V. 12 ,auch nicht mehr das Motiv der Trennung aus V. 6 aufrechterhalten. Es kann ... nun nicht mehr die Rede davon sein, daß die Leser sich von denen, die unordentlich wandeln, zurückziehen sollen, sondern nun wird erst einmal das genaue Gegenteil von ihnen erwartet: eine Hinwendung zu gerade diesen Leuten.“

438 Das spricht freilich stark gegen die von manchen Exegeten (v. a. Marxsen, Thessalonicherbrief, 101) angenommene stark unsystematische Erörterung in diesem Abschnitt. Interessant

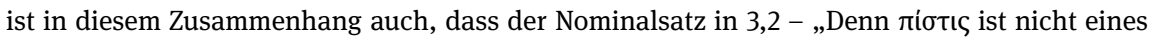

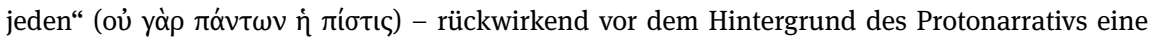
kommunikative Nebenfunktion bekommt. Es handelt sich hierbei nicht nur um ein Wortspiel, 
Was $\pi \alpha \rho \alpha y \gamma \varepsilon \dot{ } \lambda \lambda \omega \mathrm{im}$ 1. Timotheusbrief angeht, so ist bereits die erste Verwendung in 1. Tim 1,3 äußerst auffällig: Die in der Vergangenheit - nämlich im Zusammenhang der Abreise des Paulus nach Mazedonien (лорєvó $\mu \varepsilon v o \varsigma$

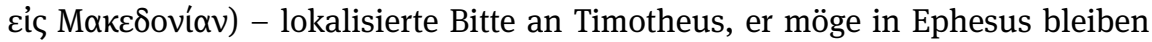

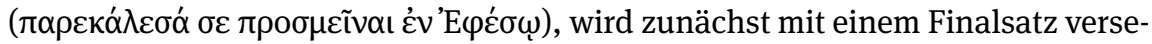

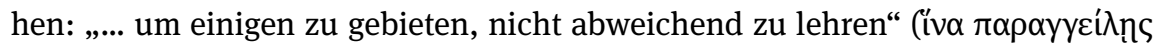

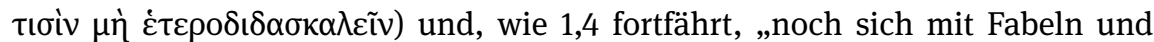

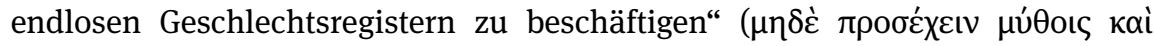

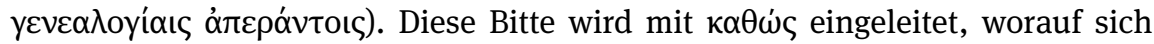
jedoch kein Gegenstück bezieht. ${ }^{439}$ Der damalige Auftrag zum Gebieten bleibt auf diese Weise auch für die Gegenwart zumindest unabgelöst. ${ }^{440}$

Eine gewisse Plausibilisierung der Auslegung, die in 1,3 eine explizite Betonung des anhaltenden Auftrags an Timotheus sieht, könnte der Gebrauch von

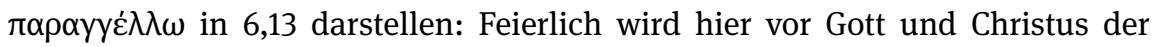

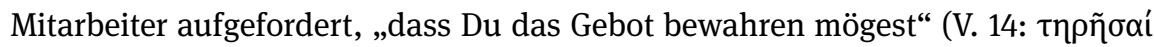

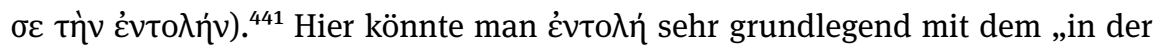

das halbwegs den Textfluss ermöglicht (so in etwa Trilling, Brief, 137). Vielmehr ist es so, dass es an dieser Stelle zwar einerseits die zuvor als Zweck markierte Errettung von den bösen und

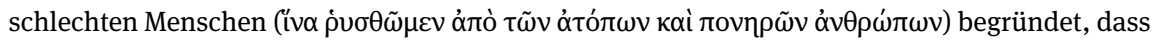

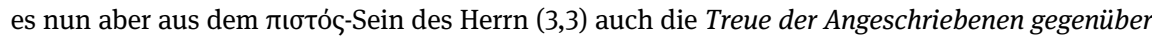
den apostolischen Befehlen folgert (3,4). Die Treue des Herrn in 3,3, „vor dem Bösen zu bewahren“

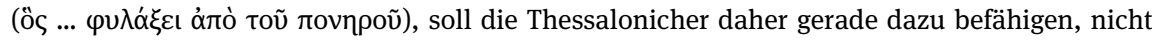
etwa selbst „untreu“ zu werden, sodass auch sie zu den „nicht allen“ in 3,2 gerechnet werden

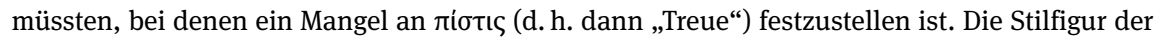
Litotes in 3,2 hat damit auch ein drohendes Potenzial, indem sie einen alternativen Ausgang des in dieser Passage skizzierten Protonarrativs aufwirft.

439 BDR $467^{2}$ meinen, die Konstruktion münde ,in ein reines Wirrsal ... infolge der unaufhörlichen Einschiebungen und Anhängsel." Das scheint nun allerdings wiederum etwas übertrieben, denn mit V. 5 beginnt schließlich schon wieder ein neuer Hauptsatz.

440 Roloff, Brief, 62 möchte wohl etwas zu viel aus diesem Anakoluth ableiten, wenn er meint, „indem“ die Vergleichskonjunktion ohne Korrelat bleibe, werde deutlich: „Nicht darum geht es, daß der damalige Befehl des Paulus durch einen neuen ergänzt und weitergeführt würde, sondern darum, daß der damalige Befehl weiter in Geltung bleibt.“ Während der erste Teil dieser Schlussfolgerung zweifelsohne gerechtfertigt scheint, geht die zweite Hälfte über die Evidenz hinaus. Entsprechend kritisch ist auch die Fortsetzung zu sehen, wonach $\kappa \alpha \theta \omega \dot{~}$ „,begründenen Sinn“ habe und Timotheus so „als der am Ort stehengebliebene Missionar“ skizziert werde, womit „V3 die Situation des gesamten 1Tim“ beschreibe.

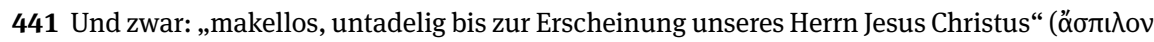

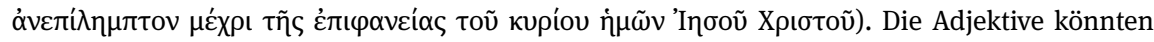
sowohl Objektsartergänzungen oder auf das Subjekt des AcI bezogen sein. Geht man vom erstge-

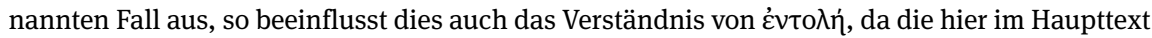
diskutierte Gleichsetzung mit den vorangehenden Aufforderungen dann schwerlich aufrechter- 
Ordination vermittelte[n] Amtsauftrag“ gleichsetzen. ${ }^{442}$ (Allerdings scheint ein Rückbezug auf die Imperative in 6,11-12 $2^{443}$ ebenso denkbar.)

Wie schon $\pi \alpha \rho \alpha y \gamma \varepsilon \dot{\varepsilon} \lambda \lambda \omega$ in 1 . Tim 1,3, so erscheint auch der durative Impe-

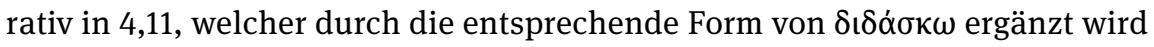

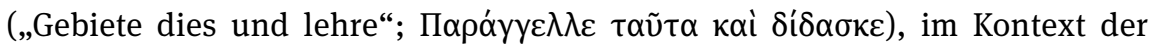
Außenandersetzung mit falschen Lehren. Worin das Gebieten genau besteht, bleibt im Abschnitt recht offen. Am ehesten findet man in der Anweisung, die in V. 7 an Timotheus selbst ergeht, wiederum ein Gebot, wie man es sich auch als von ihm ausgehend vorstellen könnte: Das „abweisen“ der „Mythen“ ( $\mu$ v́Өı) meint wohl nicht zuletzt auch das verbale Einschreiten gegen deren Verbreitung. ${ }^{444}$ Die Offenheit der Formulierung ist jedoch auch typisch für

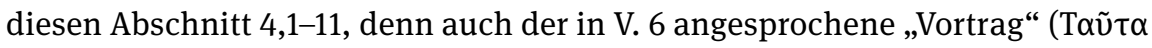

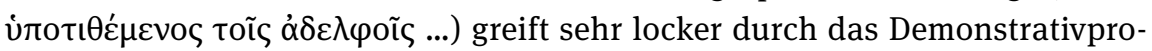
nomen auf die Verse 3-5 zurück, welche bis zu diesem Punkt lediglich als recht ausufernde Beschreibung der Speisen in V. 3 in Erscheinung getreten waren. Der genaue Inhalt der drei genannten verbalen Akte ist daher wohl auch allgemeiner dem „Material“ des Textes zu entnehmen.

Dies erklärt wohl auch den Imperativ in 1. Tim 5,7: „Und dies gebiete ...“ (каі $\tau \alpha \tilde{u} \tau \alpha \pi \alpha \rho \alpha ́ \gamma \gamma \varepsilon \lambda \lambda \varepsilon)$. Es ist nicht nötig, hier von einem allgemeineren „Kundtun“ zu sprechen, nur weil nicht unmittelbar Gebote expliziert werden. ${ }^{445}$ Der Finalsatz macht klar, wer die Empfänger der Äußerungen sind: „... damit sie untade-

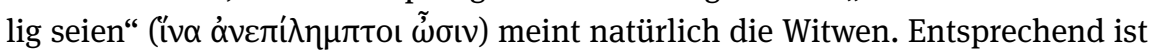
dabei wohl an solche Anweisungen gedacht, die zu einem Verhalten auffordern, wie es in V. 5 beschrieben wird, beziehungsweise gegen das in V. 6 genannte Führen eines üppigen Lebens abzielen. ${ }^{446}$

halten kann. Siehe in diesem Sinne Roloff, Brief, 352, der jedoch übersieht, dass im Falle eines Bezugs auf $\sigma \varepsilon$ ebenfalls ein Bezug auf „ein menschliches Verhalten“ gegeben wäre.

442 So Roloff, Brief, 352. Vgl. die letzte Fußnote.

443 Siehe oben, Abschnitt 4.4.2.2 zu diesem Text.

444 In diesem Sinne wohl auch Roloff, Brief, 248.

445 Vgl. die Übersetzung bei Roloff, Brief, 382: „Auch das verkündige ...“

446 Eventuell könnte man auch bereits in V. 4 eine explizite Aufforderung in Form eines Imperativs anklingen sehen. Das Subjekt sind hier aber doch wohl eher die Enkel und Kinder und nicht die Witwen. Ginge man doch vom letzteren Fall aus, so wäre wohl in V. 7 auch hierauf zurückgegriffen. Das ist freilich nicht der Fall in denjenigen Auslegungen, welche in V. 3 die „Besoldung“ und nicht allgemein die „Ehre“ im Blick haben und die in der Folge die „wirkliche“ Witwe vom Sonderfall derjenigen Witwe abgrenzen, die noch eine Familie hat. Dann würde mit V. 5 eine Zäsur erfolgen, über welche die Anweisung zum Gebieten in V. 7 nicht zurückgreifen würde. 
Eine Aufforderung zur Aufforderung ergeht auch in 1. Tim 6,17 an Timotheus. Der soll sich an die „im gegenwärtigen Zeitalter Reichen“ richten. ${ }^{447}$ Der

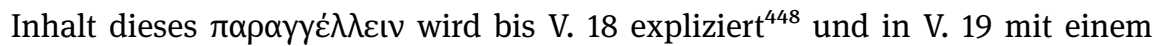
adverbialen Partizip versehen (,sich selbst einen guten Schatz sammelnd für

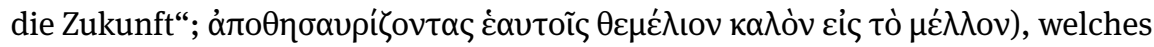
dann wiederum durch einen Finalsatz erläutert wird: „damit sie das wahre Leben

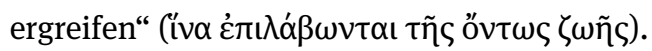

\subsubsection{Verneinte Verben des Müssens}

Bei Verben, die ein Sollen/Müssen ausdrücken, ist die Frage sehr wichtig, ob innere oder äußere Negation vorliegt. ${ }^{449}$ „Du musst nicht“ stellt keine Aufforderung dar, „you must not“ ist hingegen als negativer Befehl - als Verbot - zu verstehen. Interessanterweise scheint für ó $\varphi \varepsilon i \lambda \omega^{450}$ bei Paulus beides belegt: „Der

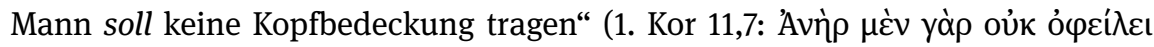

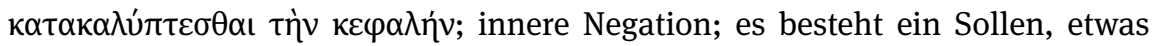
nicht zu tun), aber „die Kinder müssen nicht Schätze für die Eltern sammeln“

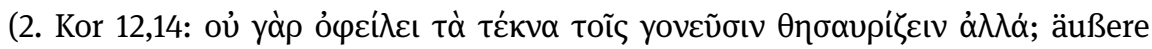
Negation; es besteht kein Sollen, etwas zu tun).

Was unpersönliche Ausdrücke der Verpflichtung angeht, so entspricht verneintes $\delta \varepsilon \tilde{~}$ dem englischen „must not“ (d. h.: „,man darf nicht“) und nicht dem deutschen „nicht müssen.“451 In 2. Tim 2,24 wird über den Knecht des Herrn daher ausgesagt, dass er die Verpflichtung hat, Streit zu unterlassen und stattdes-

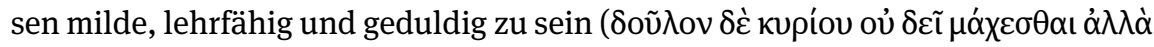

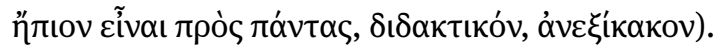

447 Zum fehlenden Artikel im Attribut siehe Robertson, Grammar, 783.

448 Und zwar:

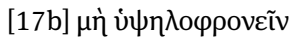

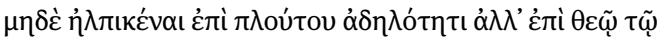

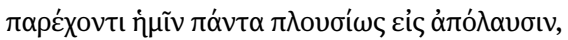

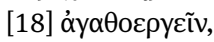

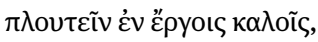

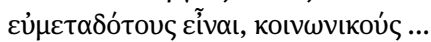

449 Im Deutschen wird die innere Negation etwa mit „sollen“ verwendet. Hier wird anders als z. B. bei „dürfen“ nicht das Hilfsverb selbst negiert, sondern der abhängige Sachverhalt. Entsprechend haben „Du sollst nicht töten“ und „Du darfst nicht töten“ beide die Funktion eines Verbots (Duden 821). Obwohl „sollen“ und „müssen“ Überschneidungen aufweisen, hat „Du musst nicht töten“ aufgrund der „äußeren Negation“ (Duden 819) eine ganz andere Stoßrichtung.

450 Siehe GGNT 218e für Verben des Müssens.

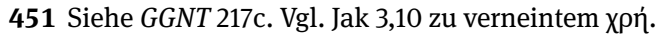




\subsubsection{Verneinte Verben des Erlaubens}

Abschließend muss noch kurz auf Verben des „Erlaubens“ eingegangen werden,

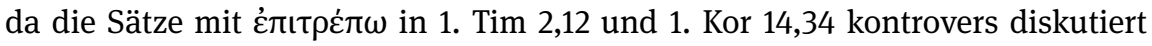
werden. Die Differenzierung zwischen äußerer und innerer Negation ist hier für unsere Fragestellung weniger relevant. Wenn Paulus in 1. Tim 2,12 sagt, er „erlaube[,] nicht[,] zu lehren/herrschen, “ so wäre hier bei innerer Negation lediglich das Zugeständnis erteilt, dass die Frauen nicht lehren/herrschen müssten.

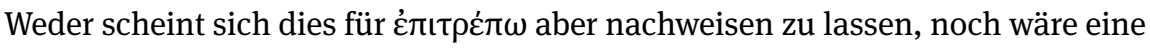
solche Aussage kontextuell angemessen. (Natürlich wäre damit auch noch keine Aufforderung ergangen, worum es uns in diesem Abschnitt ja geht.)

Allerdings ist bei Verben des Erlaubens zu beachten, dass bei entsprechender Fokus-Setzung ein verneintes Dürfen auch eine positive Aufforderung ausdrücken kann. ${ }^{452}$ Man vergleiche im Deutschen etwa den folgenden Dialog: „Darf ich den Keks noch essen?“ - „Du DARFST den Keks nicht essen, sondern ich bestehe vielmehr darauf, dass Du ihn isst.“ Auch dies ist für 1. Tim 2,12 aber sicher nicht der Fall $\left({ }^{\star}, \ldots\right.$ ich erlaube nicht, dass die Frauen herrschen(, sondern ich befehle es) ...").

Die Kategorie der Informationsstruktur scheint hier dennoch von großer Bedeutung für das Verständnis des Verses: Die - in der Exegese weitestgehend übergangene - Frage ist nämlich, ob der Fokus auf den Infinitiven liegt: Paulus würde dann betonen, dass es nicht das Lehren und Beherrschen sei, das er expli-

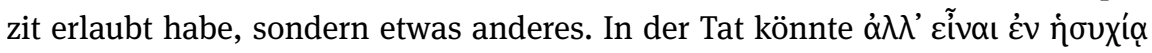
ein solches Verständnis nahelegen, da es am einfachsten als Klarstellung des

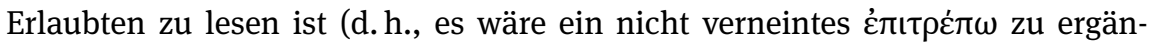
zen): ${ }^{453}$ „Es ist nicht das Lehren und Beherrschen, welches ich erlaube, sondern [ich erlaube = was ich erlaube, ist lediglich] ein Dasein in Ruhe. “454 Problematisch scheint hier vor allem die Frage, inwiefern der Imperativ in V. 11 ein solches Missverständnis eines so umfassenden Freifahrtsscheines hätte hervorrufen können. Im herkömmlichen Verständnis muss demgegenüber - auch nicht ganz

452 Siehe oben, Kapitel 4, Abschnitt 8.1.3 zur Informationsstruktur.

$453 \mathrm{Vgl}$. die oben, Abschnitt 4.4.3.7 angeführte Stelle 2. Kor 12,14, allerdings dort mit Subjekt-

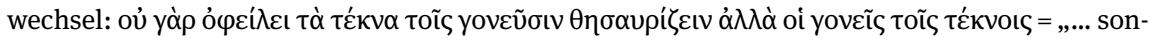
dern die Eltern [sind verpflichtet zum Sammeln von Schätzen] für die Kinder.“

454 Wright, Pastoral Letters, 22 übersetzt entsprechend: „I'm not saying that women should teach men, or try to dictate to them; rather they should be left undisturbed." Vgl. auch Wright „Women's Service“ noch paraphrasierender: „I don’t mean to imply that I'm now setting up women as the new authority over men in the same way that previously men held authority over women.“ 
unproblematisch - wohl ein Zeugma ${ }^{455}$ angenommen werden, also der Gedanke des „Sollens“ auch auf die zweite Vershälfe übertragen werden: „Eine Frau soll nicht lehren oder über einen Mann herrschen, sondern sie soll sich in der Stille aufhalten.“456

\section{Konnexionen}

\subsection{Einführung}

Das breite Spektrum der bisher besprochenen sprachlichen Gestaltungsmöglichkeiten, mit welchen Paulus zukünftiges Geschehen in sehr spezifischer Gradierung bewerten und im Hinblick auf die Sicherheit des Eintretens modulieren kann, folgte der Einteilung von Sätzen ${ }^{457}$ entsprechend ihrer kommunikativen Funktion. Dabei wurde entsprechend der syntaktischen Verwendbarkeit zwischen Hauptsätzen und Nebensätzen unterschieden, wobei im Zusammenhang mit letzterem Fall auch die oft anzutreffende alternative Konstruktion mit dem Infinitiv besprochen wurde.

Textgrammatisch betrachtet liegt bei den abhängigen Konstruktionen eine Verbindung von zwei Propositionen vor, deren inhaltliche Verbindung man als Einleitung-INHALT-Konnexion betrachten kann. ${ }^{458}$ Im Folgenden sollen zwei weitere Klassen an Konnexionen besprochen werden, die ebenfalls sehr wichtig für die Suche nach zukunftsungewissen Vorausdeutungen auf die Zukunft sind: konditionale und finale Konnexionen. ${ }^{459}$ Weshalb gerade diese beiden Konnexionen für

455 GGNT 293d. Z. B.: Elberfelder: „Ich erlaube aber einer Frau nicht zu lehren, auch nicht über den Mann zu herrschen, sondern [ich will], daß sie sich in der Stille halte.“ Alternativ könnte

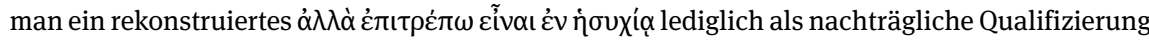
des Imperativs aus V. 11 als letztlich ja im Sinne der Frauen verstehen: „... aber immerhin erlaube ich ja, dass sie ihre Ruhe haben."

456 In 1. Kor 14,34 wird dies durch den Imperativ in der 3. Person Plural explizit gemacht: $\alpha \hat{\lambda} \lambda \grave{\alpha}$ ن்

457 Vgl. zu den unterschiedlichen Parametern in diesem Abschnitt GGNT 266a.

458 GGNT 323b. Siehe Nr. 6 in der Übersicht bei GGNT 352b.

459 Möchte man das nun in den Blick genommene Phänomen aus der Perspektive der Klassifizierung von Sätzen betrachten, stellt sich der Zusammenhang wie folgt dar: Die bisher besprochenen abhängigen Sätze können entsprechend ihrer syntaktischen Funktion als Gliedsätze eingeordnet werden (d. h. nicht als Attributsätze), da sie als Objektsatz der übergeordneten Konstruktion dienen (vgl. GGNT 270a). Der Kategorie der Gliedsätze sind außerdem noch die Subjektsätze und die Umstandsangabesätze zuzuordnen (GGNT 270a). Zwei Satztypen aus dem Bereich der Umstandsangabesätze werden im Folgenden besprochen: Konditionalsätze und Finalsätze. Diese Sätze nehmen eine adverbiale Bestimmung des Verbinhalts der übergeordneten 
die Analyse zukunftsungewisser Vorausdeutungen auf die Zukunft des Erzählers relevant sind, wird in den beiden folgenden Abschnitten jeweils einleitend dargelegt.

\subsection{Bedingte Ereignisse}

\subsubsection{Indefinite Konditionalsätze mit $\varepsilon i$}

\subsubsection{Hinführung}

Wie in der allgemeinen Einführung verschiedener Konnexionen bereits erwähnt (Kapitel 5, Abschnitt 3.2), benennt die „Wenn“-Proposition einer konditionalen Konnexion „eine sachliche Voraussetzung, “während „die ,Dann'-Proposition die damit fest $\mathrm{zu}$ verbindende sachliche Konsequenz“ ausdrückt. ${ }^{460}$

Konstruktion vor. Im ersten Fall wird aus textgrammatischer Sicht eine konditionale (GGNT 331), im zweiten Fall eine finale (GGNT 336) Konnexion hergestellt.

Für die Untersuchung der Konnexionen sind folgenden zwei Überlegungen zu beachten: Erstens, auch hier gilt - wie für andere abhängigen Sätze (s. o. zu den abhängigen Behauptungs- und Begehrsätzen) - dass Infinitiv- oder Partizipialkonstruktionen an dieselbe Stelle treten und die entsprechende Satzgliedfunktion (als adverbiale Umstandsangabe) übernehmen können. (Siehe allgemein GGNT 270d.) Entsprechend sind auch die alternativen satzwertigen Ausdrucksmöglichkeiten konditionaler (aufgeführt bei GGNT 259h) und finaler (aufgeführt bei GGNT 250j) Umstandsangaben zu berücksichtigen. Zweitens: Es muss die ebenfalls mögliche Unterteilung von abhängigen Sätzen entsprechend des verwendeten Einleitewortes in Konjunktionalsätze auf der einen Seite und Relativsätze auf der anderen Seite sorgfältig bedacht werden (vgl. zum Folgenden GGNT 270c). Offensichtlich ist, dass die Adverbialsätze aufgrund der konjunktionalen Einleitewörter (čáv, عỉ bzw. iv $\alpha$ etc.) ebenso zu den Konjunktionalsätzen zu rechnen sind, wie dies für Subjekt-Objektsätze (mit ötı bzw. İva im Fall von abhängigen Begehrsätzen) gilt. Dem steht die Kategorie der Relativsätze gegenüber, wo die einleitenden Relativpronomina und -adverbien den Nebensatz nicht nur einleiten, sondern zusätzlich auch die Rolle eines Satzglieds im Nebensatz innehaben. Syntaktisch entsprechen diese beiden Kategorien in der Regel Gliedsätzen auf der einen Seite und Attributsätzen auf der anderen Seite. Es gilt jedoch: Zum einen können auch Relativsätze teilweise als freie Umstandsangabe auftreten (GNT 289c). Zum anderen - und textgrammatisch bedeutsamer - können (attributiv verwendete) Relativsätze auch einen zusätzlichen „adverbialen Nebensinn“ aufweisen (GGNT 290). Dem entspricht auf der Gegenseite, dass etwa Finalsätze nicht immer Satzgliedfunktion haben müssen, sondern auch als Attribut auftreten können. Siehe GGNT 288b. Dasselbe gilt natürlich auch für Sätze mit ötı und ǐva: Die meisten der oben besprochenen Sätze hatten Satzgliedfunktion als Objekt (,Wen oder was weiß/will Paulus?“). D. h.: Auch der abhängige Behauptungsoder Begehrsatz kann die Funktion eines Attributs zu einem Bezugswort in der übergeordneten Konstruktion übernehmen, welches dort die Satzgliedfunktion als Objekt innehat. Vgl. GGNT 288a. An den entsprechenden Stellen wurde oben bereits darauf hingewiesen.

460 GGNT 331b. 
Weshalb ist dieser Relationstyp von Propositionen für die Suche nach zukunftsungewissen Vorausdeutungen auf die Zukunft des Erzählers bedeutsam? Handelt es sich bei besagter Konsequenz um ein aus Sprecherperspektive in der Zukunft liegendes Ereignis, so wird auf diese Weise einerseits (1) ein möglicher Handlungsfortgang einer expliziten oder impliziten Erzählung aufgegriffen, dieser aber andererseits (2) auch als lediglich potenziell - da abhängend von der Erfüllung einer Bedingung - dargestellt.

Es wurde oben auch bereits dafür argumentiert, dass der (I) indefinite Fall des Konditionalsatzes auch im Griechisch des NT ein unbestimmtes Verhältnis der Protasis zur Wirklichkeit zum Ausdruck bringt, also (entgegen der teilweise anzutreffenden Benennung als „Realis“) nicht einfach als Kausalangabe zu verstehen ist. Gerade vor dem Hintergrund der Kategorie des Protonarrativs ist die Relevanz dieser aufrechterhaltenen Unterscheidung offenkundig: Anders als der Kausalsatz markiert der Konditionalsatz die Möglichkeit einer Alternative - selbst wenn diese nur hypothetisch ist, da das in der Bedingung zum Ausdruck kommende Ereignis innerhalb des Kommunikationskontextes als tatsächlich vorausgesetzt werden kann. ${ }^{461}$ Der Konditionalsatz provoziert damit die Simulation eines alternativen Handlungsverlaufs, der als Kontrast zur nahegelegten Ereignisfolge diese auf vielfältige Weise stützen oder erläutern kann. ${ }^{462}$

Im Folgenden sollen die Futurformen im Römerbrief (zur Statistik siehe oben, Abschnitt 3.1) vor diesem Hintergrund besprochen werden, die innerhalb der Apodosis eines infiniten Konditionalgefüges erscheinen, also Fälle, in denen ein als unbestimmt sicher dargestellter gegenwärtiger oder vergangener Sachverhalt die Realisierung eines zukünftigen Geschehens beeinflusst. ${ }^{463}$

461 Die beiden Teile einer konditionalen Konnexion können natürlich nicht automatisch ausgetauscht werden (GGNT 331d). Der Fokus auf das „Wenn“ wirft jedoch zwangsläufig die Frage auf: „Und wenn nicht?“

462 Vgl. auch Wallace, Grammar, 680-712 - welcher diesen Typ Konditionalsatz dadurch charakterisiert sieht, dass in ihm die Bedingung „for the sake of the argument“ als gegeben vorausgesetzt würde - im Hinblick auf 1. Thess 4,14 (S. 694): „Many modern translations render the particle since. Although it is certainly true that Paul embraced this as true, to translate it as since keeps the audience at an arm's length. The sentence becomes a lecture rather than a dialogue. By translating it if, the audience is drawn into the argument of the apodosis. Their response would be something like, ,If we believe that Jesus died and rose again? Of course we believe that! You mean that this indicates that the dead in Christ will not miss out on the rapture?' In such instances it is not the protasis that is in doubt, but the apodosis. (Further, to say that the connection is merely logical hardly does such texts justice.) Not infrequently in the NT, the speaker draws his audience to just such a connection, basing his argument on what both speaker and audience already embrace as true. These instances are not without exegetical significance.“

463 Siehe auch oben, Kapitel 12, Abschnitt 7.7 zur Diskussion indefiniter Konditionalsätze im Zusammenhang mit dem Diserzählen. 
Möglich wäre natürlich auch der umgekehrte Fall, dass also eine gegenwärtige oder vergangene Situation (meist wohl ein Zustand) abhängig gemacht würde von einem zukünftigen Ereignis. Dieses Muster liegt der paulinischen Interaktion mit den Lesern an einigen Stellen zugrunde (vgl. etwa Kapitel 12, Abschnitt $7.8 \mathrm{zu}$ 2. Kor 13,5), wird jedoch nie durch eine Konditionalperiode mit besagter syntaktischer Konfiguration, also Futur ausschließlich in der Protasis, ${ }^{464}$ realisiert. ${ }^{465}$

Eine weitere Möglichkeit, in einem Konditionalgefüge Zukunftsbezug vorzufinden, ohne dass die Apodosis eine Verbform des Indikativs des Futurs vorliegt, besteht dann, wenn vom Kontext- oder Weltwissen her ein Ausdruck mit einer futurischen Referenz verbunden werden kann. Zu erwägen ist dies etwa für 1. Kor 9,17, wo auf präsentische Protasis ${ }^{466}$ die knappe Formulierung folgt: „... dann

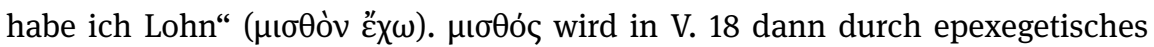
iv $\alpha$ expliziert: ${ }^{467}$ Der „Lohn“ besteht darin, freiwillig auf den Lohn zu verzichten

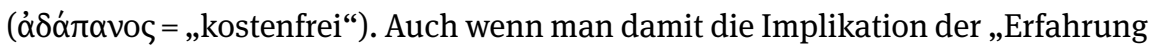
von Freiheit und Liebe hier und jetzt" verbindet, bedeutet dies nicht, dass damit eine „eschatologische“ Dimension nicht im Blick wäre. ${ }^{468}$

\subsubsection{Futur in der Apodosis im Römerbrief}

Was nun aber die eingangs erwähnten Konditionalperioden mit indefinitem Konditionalsatz und Futurverb in der korrespondierenden Apodosis angeht, liegt im Römerbrief eine solche in Röm 5,10 vor (siehe Abb. 57). Die Bedingung ist offensichtlich erfüllt. Denn dass die Versöhnung stattfand ,als wir (noch) Feinde

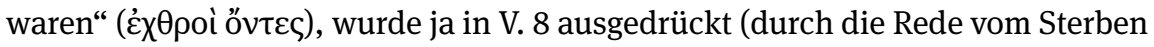
Jesus für „uns“ als Sünder). Und wie oben (Kapitel 13, Abschnitt 2.1) diskutiert,

464 Wie unten noch zu sehen sein wird liegt in 1. Kor 3,14-15 und 2. Tim 2,12 der seltene Fall vor, dass nicht nur in der Protasis eine Futurform erscheint, sondern auch in der Apodosis, dass also sowohl Bedingung als auch Bedingtes noch in der Zukunft liegt.

465 Am ehesten noch in 1. Kor 6,2, wobei hier die Apodosis in einem Fragesatz besteht und die Protasis zwar Zukunftsbezug aufweist, aber eben nicht aufgrund einer indikativischen Futurform. Siehe zum Vers unten, Abschnitt 5.2.1.3. Vgl. auch oben, Kapitel 13, Abschnitt 3.1 zum „Wissen.“

466 Aufgrund der Form $\pi \rho \alpha ́ \sigma \sigma \omega$ ist ausgeschlossen, dass es sich hier um einen irrealen Konditionalsatz handelt. So fälschlich Schrage, Brief II, 325. Im Hintergrund steht bei ihm offensichtlich (Fußnote 271 auf derselben Seite) ein Missverständnis des indefiniten Falls als „Realis“: Nähme man einen solchen an, so stünde angeblich der Gedanke einer Verdiensttheorie nahe - wohl weil dann (so Schrages unzutreffende Annahme) vorausgesetzt wäre, dass Paulus tatsächlich so gehandelt hätte.

467 Vgl. Abschnitt f) bei GGNT 272a.

468 So Schrage, Brief II, 327. 


\begin{tabular}{|l|l|}
\hline Protasis & Apodosis \\
\hline$\varepsilon i ் . . . \kappa \alpha \tau \eta \lambda \lambda \alpha_{\gamma} \eta \mu \varepsilon \nu \tau \tilde{\omega} \theta \varepsilon \tilde{\omega} \ldots$, & $\ldots \sigma \omega \theta \eta \sigma o ́ \mu \varepsilon \theta \alpha \ldots$ \\
\hline
\end{tabular}

Abb. 57: Die Konditionalperiode in Röm 5,10.

nimmt V. 9 darauf Bezug und kündigt mit $\pi \circ \lambda \lambda \tilde{\omega} . . . \mu \tilde{\alpha} \lambda \lambda$ ov die gewisse futurische Errettung an.

\footnotetext{
${ }^{9}$ Vielmehr nun, da wir jetzt durch sein Blut gerechtfertigt sind, werden wir durch ihn vom Zorn gerettet werden.

${ }^{10}$ Denn wenn wir, als wir Feinde waren, mit Gott versöhnt wurden durch den Tod seines Sohnes, so werden wir viel mehr, da wir versöhnt sind, durch sein Leben gerettet werden. ${ }^{469}$
}

V. 10 trägt nun dreierlei bei:

(1) Nach dem Fokus auf die Liebe Gottes (V. 8a) und auf die Hingabe Christi

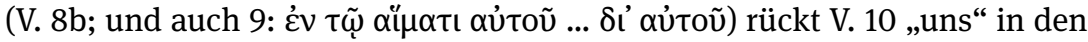
Fokus. Zwar wird die versprochene Errettung nicht von einem bestimmten Verhalten abhängig gemacht, doch streicht die Konditionalperiode heraus, dass diese Zukunft eben auch mit der Gegenwart der Menschen verbunden ist. In V. 8 klang das noch gar nicht oder höchstens in der Referenz des Perso-

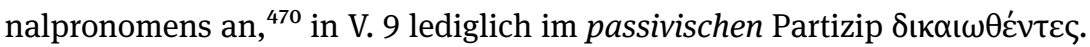
Demgegenüber weist V. 10 auf die Möglichkeit hin, dass die zukünftige Situation auch ganz anders aussehen könnte, wenn diese Voraussetzung des Übergangs vom Sünder zum Gerechten nicht gegeben wäre.

(2) Zweitens - und in der Regel von Exegeten übersehen - geht die Konditionalperiode in V. 10 aber auch noch in anderer Weise über V. 9 hinaus. Es handelt sich keinesfalls um die „Wiederholung des gleichen Schlusses.“471 Dies zeigt bereits der Konnektor yóp, der freilich von den meisten Auslegern als „nicht eigentlich begründen[d], sondern erklären[d] “ neutralisiert wird. ${ }^{472}$ Demgegenüber muss an einer kausalen Konnexion festgehalten werden - wobei hier eben nicht wie im typischen Fall des Grund-FOLGE-Musters ausschließlich an einen „ursächlichen Grund“ zu denken ist. Vielmehr liegt hier Äußerungs-

469 Revidierte Elberfelder.

470 D. h., sühnetheologisch ist die Frage, ob in V. 8 die sündige Menschheit als Ganze im Blick ist oder ob hier die Gläubigen als ehemalige Sünder auf ihre Vergangenheit schauen.

471 Wilckens, Brief I, 298.

472 So Schlier, Römerbrief, 155. 
bezug vor: ${ }^{473}$ Vers 10 begründet, weshalb die Folgerung von V. 8-9 berechtigt ist. Vergleicht man die Struktur der beiden Textteile, wird deutlich, dass V. 10

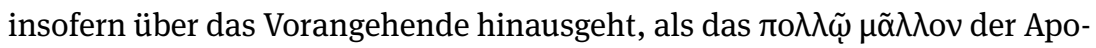

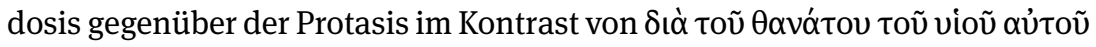

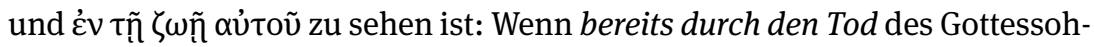
nes (vgl. metonymes $\alpha \tilde{i} \mu \alpha$ in V. 9) Versöhnung der damaligen Feinde geschah, wie viel mehr wird dann erst sein Leben die Versöhnten ergreifen! ${ }^{474}$

(3) Schließlich ist noch darauf hinzuweisen, dass der Schluss sich in rein formaler Weise bereits deutlich von den Versen 8-9 abgrenzt - und diese auf diese Weise rhetorisch äußerst geschickt ergänzt. In V. 8-9 wird der Akt der Liebe Gottes/Christi erst indikativisch konstatiert und dann nochmals durch das Partizip aufgegriffen. Die Folgerung, welche auf die Zukunft abzielt, ergibt sich lediglich indirekt über einen Vergleich der jeweils notwendigen Liebe. In V. 10 wiederum ist die Bedingung der bereits ergangenen Heilstat indefinit gelassen, wird vom Leser aufgrund von 8-9 jedoch vorausgesetzt, während die futurische Errettung in der Apodosis erscheint - und damit als logisch notwendig.

In Röm 5,17 begegnet ebenfalls eine erfüllte Bedingung im Konditionalsatz: Dass durch die Übertretung des Einen der Tod die Herrschaft angetreten ${ }^{475}$ hat ( $\tau \tilde{\omega}$

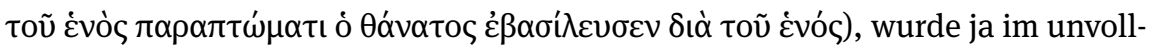

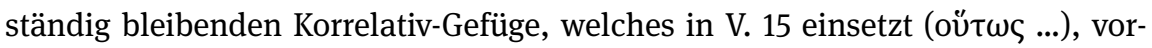
ausgesetzt. Wieder verstärkt $\pi 0 \lambda \lambda \tilde{\omega} \mu \tilde{\alpha} \lambda \lambda$ ov die schon syntaktisch vorgegebene Notwendigkeit der Folge: Die Empfänger von Gnade und Gerechtigkeit „werden

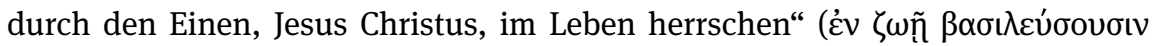

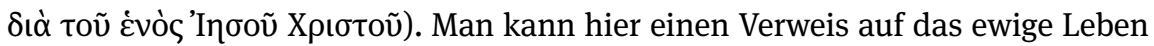
sehen. ${ }^{476}$ Und in der Tat ergibt sich aus dieser eschatologischen Zukunftsperspektive kein Widerspruch zum in V. 15 für die Vergangenheit ausgesagten Über-

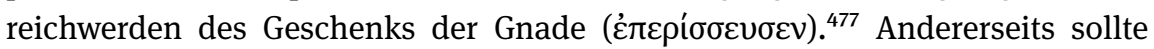
die bereits oben diskutierte (Kapitel 13, Abschnitt 2.1) Verwendungsweise des uneingeschränkt gebrauchten Futurs in 5,19 hier auch für eine weitere Option sensibilisieren: Die überreich zuteil gewordene Gnade (V. 15) wirkt eine - aus

473 Siehe Duden 1755 für eine ausführliche Erläuterung. Für konditionale Konnexionen dieser Art im Griechischen siehe GGNT 333d. Vgl. auch oben, Kapitel 6, Abschnitt 2.3.3.

474 NSS 915 bestimmt ćv daher ganz richtig: „durch sein Leben, d. h. der auferweckte Christus tritt als der Lebendige für uns ein.“

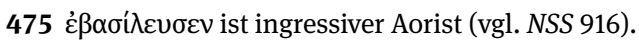

476 So etwa Wilckens, Brief I, 325.

477 So, mit Nachdruck, Wilckens, Brief I, 325. 
dieser in der Vergangenheit zu verortenden Perspektive futurische - Befähigung zum Herrschen im gegenwärtigen Leben. ${ }^{478}$

Das Konditionalgefüge mit indefiniter Protasis wird auch in 6,5 mit dem Zweck verwendet, der auch dem Verhältnis von 5,10 im Vergleich zu 5,8-9 zugrunde lag. Dass „wir“ Jesus - durch die Taufe - im Tod gleich geworden sind (бú $\mu \varphi v \tau o$ เ

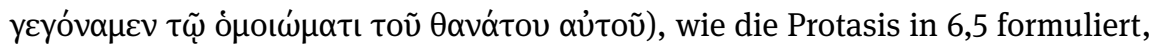
ist ja zuvor durch die Verse 3-4 etabliert worden. Was in V. 4 allerdings lediglich als Finalangabe (siehe zu deren Status unten, Abschnitt 5.3) thematisiert

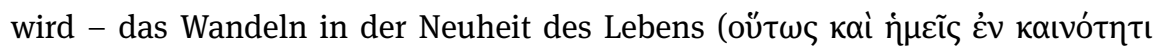
$\zeta \omega \tilde{n} \varsigma \pi \varepsilon \rho \iota \pi \alpha \tau \eta \dot{\sigma} \sigma \mu \varepsilon v$ ) - das wird in V. 5 in der Apodosis (durch $\alpha \lambda \lambda \alpha \dot{\alpha}$ nochmals verstärkt) ${ }^{479}$ als logisch notwendige Folge ausgedrückt: „wir werden sicherlich

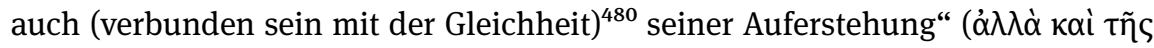

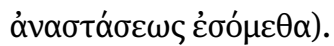

Die konditionale Konnexion bringt also die Handlung tatsächlich weiter, auch wenn durch den Bedingungssatz zugleich auf die Möglichkeit eines alternativen Plots verwiesen wird, für den Fall, dass das in 6,3 mit $\beta \alpha \pi \tau i \zeta \omega$ angesprochene Ereignis nicht vorausgesetzt werden kann. Es ist dabei vermutlich weniger ein Vergleich mit Nichtgetauften impliziert, als vielmehr dazu ermutigt, die eigene Lebensgeschichte hypothetisch ohne dieses einschneidende Erlebnis in der Vergangenheit weiterzudenken. Was in den Folgeversen aus der Voraussetzung von 6,3-4 abgeleitet wird, steht folglich im ständigen Kontrast zu diesem Protonarrativ.

Man würde nun, nach dem Konditionalgefüge in 6,5, eine Explikation dieser Folgerung erwarten, was es also bedeutet, Anteil an der Auferstehung Jesu zu haben. Doch zunächst expliziert 6,6 erstmal nur die Protasis, indem auf das Wissen rekurriert wird, dass der alte Mensch mitgekreuzigt und der Leib der Sünde abgetan sei. Dies geschieht wohl, weil durch das Motiv des Todes in 6,7 die Konsequenz dieses Todes erläutert werden kann: Freiheit von der Sünde. ${ }^{481}$

Mit 6,8 beginnt ein zweiter Anlauf, in welchem nun die positive Konsequenz entfaltet werden soll. Daher wird die Protasis aus 6,5 nur in geraffter Form auf-

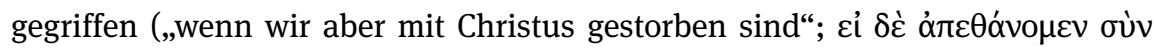

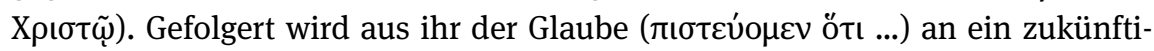

478 Auch wenn man unter dem „Leben“ das ewige versteht, so ist eine solche Deutung nicht ausgeschlossen. $\dot{\varepsilon} v \zeta \omega \tilde{n}$ wäre dann eben nicht mehr die Sphäre des Herrschens, sondern Instrumentalangabe. Eine mögliche Parallele bietet Röm 6,4, wo in der eigentümlichen Konstrukti-

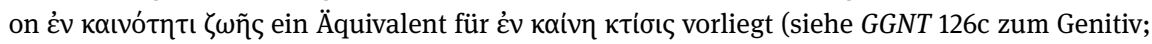
vgl. NSS 917).

479 NSS 917.

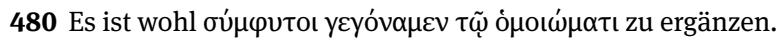

481 Zum traditionsgeschichtlichen Hintergrund siehe Vollenweider, Freiheit, 340. 


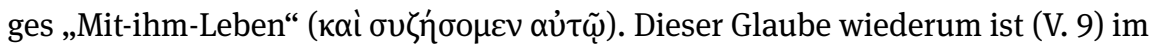

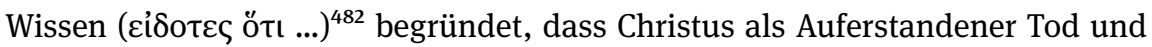
Sünde (V. 10a) endgültig überwunden hat und daher ganz für Gott lebt (V. 10b). So wie aus der Gleichheit mit dem Tod Christi der Tod gegenüber der Sünde folgt, folgt aus der Verbindung mit seiner Auferstehung daher ein Leben für Gott.

Genau diese doppelte Ausrichtung liegt natürlich dann in der Aufforderung in 6,11 (erläutert in 6,12-13) vor: „Haltet euch der Sünde für tot, Gott aber lebend in Christus!“ Sofern die Römer dieser Aufforderung folgen werden, wird in ihrem zukünftigen Lebenswandel die Auferstehung Christi $(6,5)$ beziehungsweise das „Leben mit ihm“ $(6,8)$ zum Ausdruck kommen.

Der Abschnitt fokussiert daher zweifellos auf die Zukunft der Angesprochenen, also auf ihr Handeln nach und in Reaktion auf das Gelesene. Fragt man nach dem genauen temporalen Bezug der Futurformen in der Apodosis von 6,5 und der von 6,8, so wird man allerdings dennoch vorsichtig sein müssen, diese komplett auf die potenzielle Befolgung der Aufforderung zu beschränken.

Wie bereits erwähnt, impliziert die Tatsache, dass Paulus ein konditionales Gefüge wählt, einen Abgleich des von ihm skizzierten Handlungsverlauf mit einer alternativen Erzählung, deren Plot von einer linearen Fortführung des der Bekehrung vorausgehenden Verhaltens (einer „passiven Prognose“) geprägt ist.

Die hier ins Auge gefasste Zukunft ist folglich die Zukunft, die vor dem Täufling liegt. Auch die abschließende verneinte futurische Aussage in 6,14 - „die

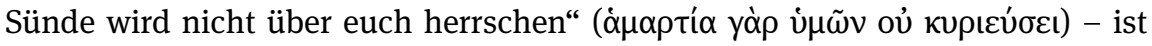

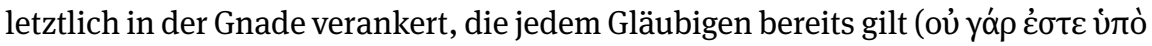

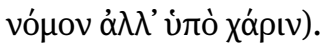

Die angesprochenen Römer bekommen hier also nicht einfach eine Geschichte „berichtet“ (vgl. Kapitel 8, Abschnitt 4 zur Textfunktion). Gerade durch die Konditionalperioden und den Knotenpunkt der Taufe sind sie dazu aufgefordert, die im Text angelegte Handlungsfolge mit ihrer je eigenen Biographie abzugleichen. Es ist dabei Paulus' Hoffnung, dass sich das Protonarrativ einer passiven Prognose auf der Grundlage des vormaligen Zustandes im Rückblick als lediglich, mit Ryan gesprochen, „virtuell“ (Kapitel 10, Abschnitt 3) herausstellt, die beiden Handlungsstränge also deutlich auseinanderklaffen.

Die implizite Kontrasterzählung stützt also die Aufforderungen in 6,11-13 einerseits, indem die römischen Gläubigen die Divergenz zwischen den beiden Handlungssträngen feststellen und sich dadurch auf ihrem bisherigen Weg bestätigt fühlen sollen, andererseits dadurch, dass die Lektüre des Textes ihnen

482 Zur Frage einer tatsächlich von Paulus mit den Römern geteilten Tradition siehe Vollenweider, Freiheit, 328. 
jetzt eine neue Gelegenheit bietet, eine Anpassung des durch ihr Leben gebildeten Handlungsstranges vorzunehmen. Siehe auch oben (Abschnitt 4.4.2) zur polyvalenten Funktion des durativen Imperativs in V. 13, welcher je nach im Einzelfall vorliegendem Verhältnis von passiver Prognose und tatsächlicher Biographie unterschiedlich in das individuelle Protonarrativ eingebaut werden kann.

Indefinite Konditionalsätze mit ganz ähnlicher Thematik erscheinen auch in Röm 8,9-11, wobei die Futurform in V. 11 vermutlich (und gegen etwa Calvin) hier die endzeitliche Auferstehung im Blick hat. ${ }^{483}$ Hier finden sich sogar - in 8,9 - eine explizite Andeutung der „Alternativhandlung“: „Wenn jemand den

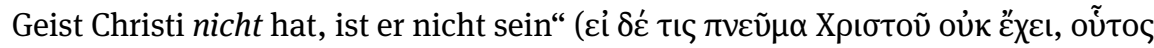

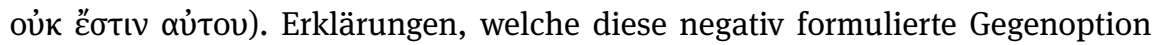
als ein Überbleibsel von liturgischen „Exkommunikationsformeln“ erachten, ${ }^{484}$ greifen ebenso zu kurz, wie die Versicherung, der Zweck sei hier „the positive one of asserting that every Christian is indwelt by the Spirit. “485 Dies gilt nicht nur angesichts des zum indefiniten Fall des Konditionalsatzes allgemein Gesagten, sondern auch umso mehr, da hier mit tıৎ zusätzlich ein verallgemeinerndes Element eingebracht wird, ${ }^{486}$ welches das Subjekt vom ن் $\mu \varepsilon \tilde{\varsigma}$ der Versicherung, „im Geist“ zu sein, abhebt.

Die explizite Nennung der Alternativoption in V. 9 unterstreicht daher die ohnehin gegebene Funktion des indefiniten Konditionalsatzes, die Leser zur Bildung von hypothetischen Alternativ-Protonarrativen $\mathrm{zu}$ ermutigen. Dieses lesersteuernde Element beeinflusst die Lektüre der Konditionalperioden bis V. 11, wo dann eine futurische Indikativ-Form erscheint.

Ebenso an die bisher diskutierten Fälle erinnert die Tatsache, dass auch hier die Konditionalperiode in V. 11 den Argumentationsfluss vorantreibt: In V. 9a hatte Paulus den Römern zugesprochen ,im Geist“ zu sein, sofern der Geist Gottes in ihnen wohne. In der negativ formulierten Alternative wird daraus der „Geist Christi.“ Das erlaubt Paulus in V. 10, in der Protasis vom Sein Christi „in euch“ zu sprechen.

Die Konditionalperiode in V. 11 nimmt dann die Apodosis aus V. 10 auf, in welcher in einer sehr dichten Formulierung die Rede davon ist, dass der Körper aufgrund der Sünde zwar „tot“ sei, der Geist aber Leben wegen Gerechtigkeit

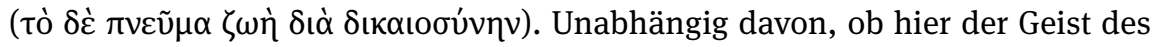

483 Wilckens, Brief II, 133 schreibt: „wie 6,5.“ Doch sind die Signale im Text doch sehr unterschiedlich deutlich in beiden Passagen. Vieles hängt natürlich davon ab, wie man die präsenti-

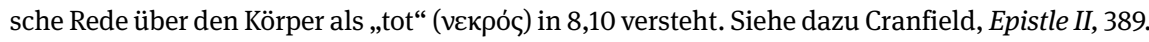
484 Vgl. Wilckens, Brief II, 131.

485 Cranfield, Epistle II, 388.

486 Siehe auch GGNT 255f. 
Menschen und/oder der Geist Christi/Gottes gemeint ist, erfordert der Fortgang, dass mit $\zeta \omega \eta ́$ eine eschatologische Perspektive eingenommen ist. Denn die Periode in 8,11 expliziert, weshalb der im Konditionalgefüge von 8,10 implizierte Zusammenhang gilt: Der innewohnende Geist taucht nun in der Protasis als derjenige „dessen, der Jesus aus den Toten auferweckt hat“ (

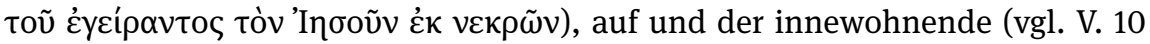

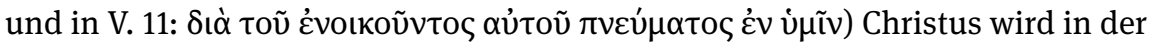

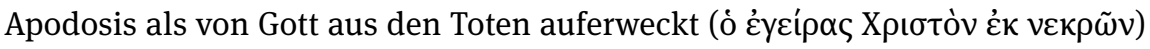
charakterisiert.

In auffälliger Weise wird nun also das historische Ereignis der Auferstehung eingebunden in eine Ankündigung des zukünftigen Wieder-lebendig-Machens

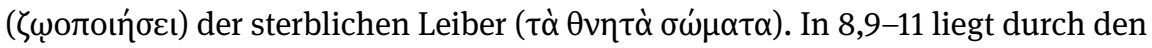
ausführlichen Gebrauch des Konditionalgefüges, die Explizierung der Alternative und die Einbindung eines vergangenen Ereignisses somit eine besonders betonte Aufforderung an die Leserschaft vor, sich die Zusammenhänge in narrativer Gestalt $z u$ vergegenwärtigen.

In 8,12 fährt Paulus dann völlig ohne Einschränkung fort, wenn er betont, dass „folglich“ unsere Schuldigkeit nicht gegenüber dem Fleisch bestünde. Denn unter der Voraussetzung, dass die Römer eben doch ó $\delta \varepsilon \lambda \varphi o i ́ ~ s i n d,{ }^{487} \mathrm{kann}$ auch davon ausgegangen werden, dass sie die aufeinander aufbauenden Bedingungen tatsächlich erfüllen.

Dass die Schuldigkeit stattdessen gegenüber dem Geist bestehe, ${ }^{488}$ wird von Paulus dann gar nicht mehr explizit gesagt, da er in 8,13 nochmals explizieren möchte, wie desaströs die Folgen des für die Christen eben nicht verpflichtenden

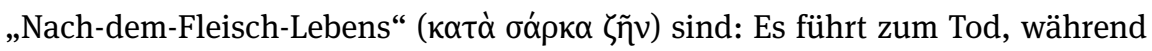
die Tötung der Taten des Leibes zum Leben führt. Die hier durch das umschrie-

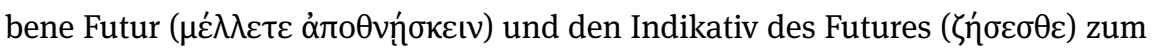
Ausdruck gebrachten Aussichten verweisen auf die beiden mit der Lebensausrichtung unmittelbar verbundenen Ziele und damit letztlich auf verschiedene eschatologische Zustände.

Nachdem die Leser in V. 12 vorläufig als „Brüder“ angesprochen wurden und somit implizit in einen der möglichen Handlungsstränge hineingenommen wurden, wird auch hier wieder auf die Möglichkeit einer Handlungsabzweigung

487 Vgl. 8,14, wo mit öбol ... wieder die Bedingung für diese Gottessohnschaft (und damit Geschwisterschaft) thematisiert wird.

488 Die Platzierung der Verneinung impliziert diesen Kontrast zwischen einer nicht gegebenen Schuldigkeit gegenüber dem Fleisch und einer tatsächlich gegebenen Schuldigkeit gegenüber Gott (vgl. 6,12-13). Vgl. Cranfield, Epistle II, 394. 
verwiesen ${ }^{489}$ - die diesmal nicht in der Vergangenheit liegt (in der durch den Glauben angeeigneten Geist-Gabe des zuvor Auferweckten), sondern auch das gegenwärtige Leben ( $\zeta \tilde{\eta} \tau \varepsilon$... $\theta \alpha v \alpha \tau o \tilde{\tau} \tau \varepsilon)$ betrifft und damit die zahlreichen Entscheidungen im konkreten Handeln (vgl. $\pi \rho \alpha \dot{\xi \xi ı c)) . ~}$

Narratologisch höchst interessant ist Röm 11,19-24 (siehe unten, Abschnitt 5.2.2.2 zum prospektiven Konditionalsatz in V. 22). In Röm 11,21a erscheint ein indefiniter Konditionalsatz, nachdem zuvor in V. 20b eine Aufforderung zur Gottesfurcht erging. Der syntaktische Zusammenhang ist hier allerdings nur mit Schwierigkeiten zu erschließen. Erwogen wird teilweise, dass es sich bei der Auf-

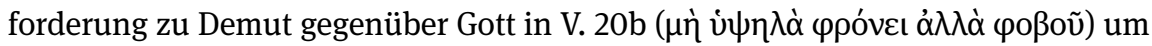
die Apodosis zur Protasis aus V. 21a handelt: „- wenn ... Gott die natürlichen

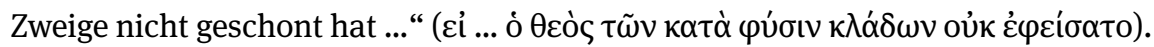
Bei dieser Auflösung wäre die Aufforderung V. 20b also letztlich in einer Analyse des heidenchristlichen Lesers seiner eigenen Situation verankert. ${ }^{490}$

Erwogen werden kann diese Lesart aufgrund der teilweise belegte Einleitung des folgenden Nebensatzes V. $21 \mathrm{~b}$ mit $\mu \eta \dot{n} \pi \omega{ }^{4}{ }^{491}$ Hält man es für ursprünglich und möchte nicht annehmen, dass hier elliptisch als eigentliche Apodosis ein „so ist

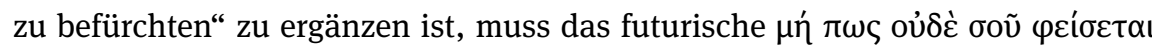
(„so wird er auch Dich vielleicht nicht schonen“) in V. 21b eigentlich zwangsläufig in freier Weise von $\varphi$ oßoṽ in V. 20b abhängig sein ${ }^{492}$ (was auch gut zum Futur passen würde). ${ }^{493}$ Dadurch würde V. 21b als Apodosis des Konditionalgefüges nicht mehr in Frage kommen und diese syntaktische Rolle müsste anderweitig gefüllt werden. Die Aufforderung in V. 20b scheint hierfür der einzige verbliebene Kandidat. Hält man diese Textvariante jedoch für sekundär, so wäre in V. 21b im Ind. Fut. keine Befürchtung geäußert, sondern die logische Folge der (vom

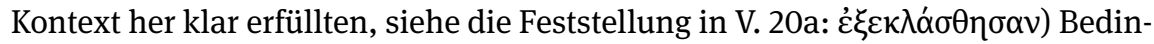
gung aus V.21a angegeben.

Für die Gestalt des hier implizierten Protonarrativs ist die exegetische Entscheidung in dieser Sache daher durchaus wichtig, auch wenn Kommentatoren in der Regel die beiden Varianten nicht ausführlich diskutieren, auch wenn man kaum - wie anscheinend oft vorausgesetzt - sowohl $\mu$ ń $\pi \omega$ s für ursprünglich halten kann und zugleich diesen Teil des Verses als Apodosis verstehen kann.

Insbesondere die Semantik des Verbs $\varphi \varepsilon i \delta$ ठo $\mu \alpha$ I wird von ihnen erstaunlicherweise kaum vor dem Hintergrund der beiden syntaktischen Optionen diskutiert.

489 Vgl. GGNT 281b.

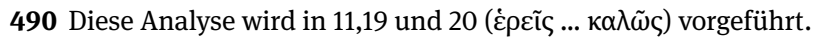

491 Vgl. oben, Abschnitt 4.3 zu den Befürchtungssätzen.

492 Vgl. NSS 936.

493 Vgl. zum Futur in den verschiedenen Rekonstruktionen der Syntax BDR $370^{3}$. 
Es scheint auf jeden Fall auch mit einer Auflösung des Futurverbs als Apodosis kompatibel zu sein, wenn man von der Bedeutung ,dafür sorgen, dass jemandem Schwierigkeiten erspart bleiben' ausgeht: ${ }^{494}$ Wenn Gott bei seinem eigenen Gewächs streng vorging (V. 21a), dann wird er wohl auch dem „Aufgepropften“ keine Sonderbehandlung angedeihen lassen (V. 21b).

Allerdings könnte man aufgrund des frühchristlichen Sprachgebrauches eher davon ausgehen, dass mit dem Verb auf eine aktivere Handlung Bezug genommen ist, dass nämlich ein tatsächlich anzunehmendes schreckliches Schicksal abgewendet wird. ${ }^{495}$ Im Umkehrschluss wäre durch die Verneinung natürlich hier eine weitaus schärfere Androhung vorgenommen: Es ginge nicht darum, dass Gott auch „streng“ mit dem Angesprochenen umgehen werde, vielmehr würde einer tatsächlich bestehenden Bedrohung freie Hand gelassen. Man könnte daher argumentieren, dass es sich hierbei plausibler um eine Furcht als um eine unvermeidbare Folge einer vom Kontext her ja klar erfüllten Bedingung handeln dürfte.

Manche Übersetzungen nehmen diese Schwierigkeit wahr. So formuliert die NGÜ als Fragesatz und mit modalem Futur: „Denn wenn Gott die natürlichen Zweige nicht verschont hat, warum sollte er dann dich verschonen?“ Auch das eventuell sekundäre $\mu$ ń $\pi \omega \varsigma$ wäre aus dieser - ganz richtigen - Empfindung heraus zu erklären. Versteht man das Verb im skizzierten Sinn, so wird hier doch letztlich das „Herausbrechen“ aus V. 20a als unvermeidbares Schicksal dargestellt. Es ist also bei der Lesart ohne $\mu$ ' $\pi \omega \varsigma$ von einem sehr allgemeinen Verständnis des Verschonens auszugehen und man darf die Referenz des NichtVerschonens - das Herausbrechen der Zweige in V. 20a - nicht mit in die Verbsemantik eintragen.

Dass dies die richtige Interpretation sein muss und die Protasis in V. 21a mit V. 21b als Apodosis zu verbinden ist, zeigt letztlich das auf $\varepsilon$ i folgende yó $\rho$, welches andernfalls nicht zufriedenstellend erklärt werden kann. Es markiert die gesamte Konditionalperiode als begründendes Element einer Konnexion. Das zeigt etwa das ebenfalls mit oủ $\delta$ in der Apodosis konstruierte Konditionalgefüge in 1. Kor 15,16: Nachdem V. 15 die Behauptung aufgestellt hat, dass Paulus und seine Kollegen - Verkündiger der Auferstehung Christi - sich als falsche Zeugen herausstellen würden, wenn Christus nicht von den Toten auferweckt worden wäre (V. 14), fasst V. 16 nochmal die diesem Schluss zugrundeliegende (үó $\rho$ ) Voraussetzung

494 LN 6721: ,to cause someone not to be troubled.

495 Siehe Michel, Brief, 277: „V 21 lehrt in der allegorischen Redeweise zurück: Gott, der die Zweige, die natürlich angewachsen sind, nicht von dem Gericht verschont hat, wird umso weniger die eingepfropften Zweige vom Gericht ausnehmen.“ Vgl. auch LSJ 1920,I: „spare persons and things, e.g. in war, i. e. not destroy.“ 
zusammen. Entsprechend könnte man im Deutschen den Satz „Denn wenn Tote nicht auferweckt werden, dann ist auch Christus nicht auferweckt worden“ (عi

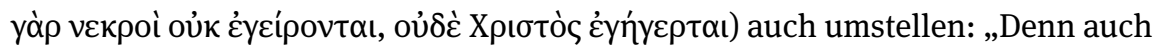
Christus wurde nicht auferweckt, wenn Tote nicht auferweckt werden.“Analog wäre man gezwungen, ein Konditionalgefüge aus der Protasis Röm 11,21a (عỉ yò $\rho$

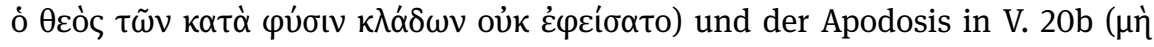

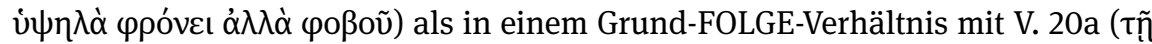

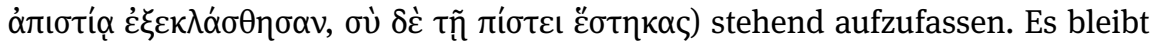
aber äußerst unklar, wie diese Behauptung durch die Aufforderung in V. 20b „begründet“ sein könnte. Vielmehr scheint es sich ja bei der Feststellung und V. 20a und der Aufforderung in V. 20b um eine asyndetische Grund-FOLGE-Beziehung zu handeln. Die Analyse der eigenen Situation führt zur Aufforderung zu einer angemessenen Einstellung - und nicht die Aufforderung zu einer demütigen Einstellung zur Erkenntnis des wahren Charakters der eigenen Situation.

Auch syntaktisch wäre die Konstruktion ausgesprochen ungewöhnlich. Denn während die Kombination ci yó $\rho$ in den Paulusbriefen $28 \mathrm{Mal}$ erscheint, ${ }^{496}$ begegnet man einem nachhängenden $\varepsilon i$-Satz innerhalb eines mit yó $\rho$ als begründend markierten Propositionsgefüge nahezu ausschließlich im Fall von Exzeptivkonditional-497 und Konzessivsätzen. ${ }^{498}$ (Dies gilt auch für Konditiongefüge mit عí ohne yó. . $^{499}$ Für ein nachklappendes ,wenn denn“ würde man wie in Röm 8,9

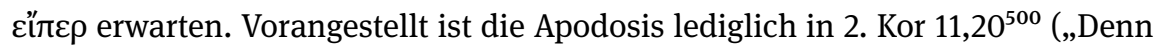
[yóp] ihr ertragt es, wenn ..."), wohl, weil daraufhin gleich 5 Bedingungen gelistet werden. ${ }^{501}$ Auch dort ist freilich das yó $\rho$ dann in Textfolge in die erste der beiden Propositionen integriert.

496 Röm 4,2.14, 5,10.15.17, 6,5, 8,13, 11,15.21.24, 14,15, 15,27, 1. Kor 2,8, 9,17, 11,6, 15,16, 2. Kor 2,2, 3,9.11, 8,12, Gal 2,18.21, 3,18.21, 6,3, Kol 2,5, 1. Thess 4,14, 2.Tim 2,11. Interessanterweise taucht die Konstruktion in den umstrittenen Paulusbriefen nur zwei Mal auf, wobei aber 2. Tim 2,11 interessanterweise im Zitat des „zuverlässigen Wortes“ ebenfalls - d. h. wie in Röm 11,21 - ein vergangenes Ereignis in der Protasis mit einem futurischen Ereignis in der Apodosis kombiniert:

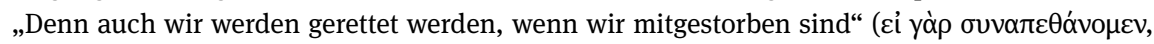

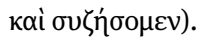

497 Röm 7,7, 13,1, 1. Kor 2,2, 1. Kor 2,11, 2. Kor 12,13.

498 2. Kor 12,11. Vgl. auch 2. Kor 7,8 (unsicheres yó $\rho)$.

499 1. Kor 15,2 ist etwa eine Ausnahme.

500 2. Kor 2,10 kann hier nicht wirklich dazu gerechnet werden, da die Protasis „Denn auch ich

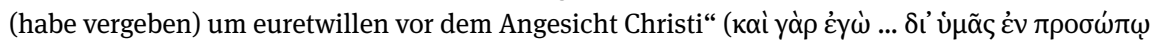

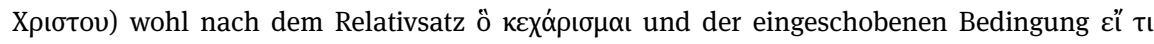

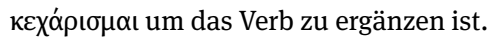

501 Vgl. allerdings auch 2. Kor 11,4 mit der Standardfolge (zwar „nur“ drei Bedingungen, diese jedoch allesamt attributiv ausgestaltet). 
Röm 11,20a-21 meint folglich nicht: „Wenn nämlich Gott die natürlichen Zweige nicht verschont hat, sei nicht hochmütig, sondern demütig! Es ist immerhin zu befürchten, dass er auch Dich nicht schonen wird!" Vielmehr ist ein Protonarrativ kreiert, das durch V. 20a als sich sehr sicher verwirklichend kommuniziert wird, das aber zugleich bewusst unkonkret bleibt: das „Nicht-Schonen“ manifestierte sich im Fall der natürlichen Zweige im Akt des Herausbrechens. Dieselbe Haltung wird auch dem Aufgepropftem gegenüber zur Anwendung kommen. Wenn freilich die Aufforderungen aus V. 20b befolgt werden, so ist dieses strenge Agieren nicht weiter problematisch, denn dann gibt es nichts, wovon man verschont zu werden bräuchte. Ein Protonarrativ, in welchem Hochmut eine Rolle spielen würde, wird somit als notwendigerweise in einem Desaster endend dargestellt. Von der hier also in der Rede vom „Nicht-Verschonen“ in den Vordergrund

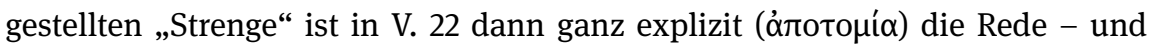
dort wird dann auch das potenzielle Ereignis, dass auch der Eingepropfte wieder „herausgeschnitten“ werden könnte, angeführt, allerdings dann eben von einer

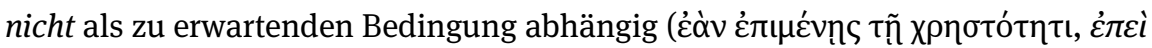

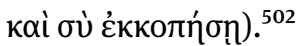

Unkontrovers ist die Zuordnung der Futur-Form „sie werden eingepfropft

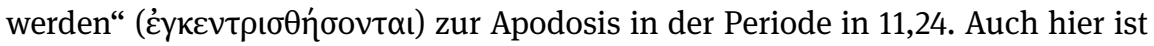
die Folge wieder mit $\pi$ ó $\omega \omega \mu \tilde{\alpha} \lambda \lambda$ ov hervorgehoben, ${ }^{503}$ die Bedingung greift zurück auf diejenige in V. 17. Dass sie vom angesprochenen ov́ für erfüllt gehalten wird, setzt Paulus nicht nur voraus, sondern hat er in Form eines Zitates in V. 19 bereits durch den Mund des Gegenübers verlauten lassen.

Interessanterweise bleibt der Bezug der Ereignisfolge zur Wirklichkeit die gesamte Passage hindurch sehr zurückhaltend formuliert: Behauptet wird lediglich in V. 16 die aus der Heiligkeit der Wurzel abgeleitete Heiligkeit der Zweige. Die Möglichkeit, dass tatsächlich einige der Zweige herausgerissen wurden,

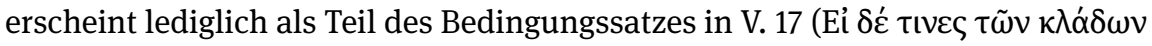

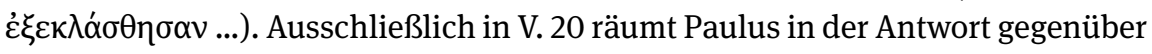
dem imaginierten Dialogpartner das Herausreißen kurz ein - um dann daraus direkt zur Unangemessenheit der Arroganz des Angesprochenen überzugehen.

Auch wenn die Ereignisfolge also dem Leser klar vor Augen steht, ist bemerkenswert, dass sie von Paulus gerade nicht explizit erzählt wird. Es bleibt ein Protonarrativ, das man zwar erzählen könnte - aber eigentlich nicht sollte. Anscheinend gibt es für Paulus eben auch eine „Ethik des Erzählens“ - und das Protonarrativ, das in V. 17 durchscheint, setzt hochmütiges Denken (V. 20:

502 Siehe dazu unten, Abschnitt 5.2.2.2 ausführlich.

503 Vgl. GGNT 178b bzw. oben, Kapitel 7, Abschnitt 2.2.1. 


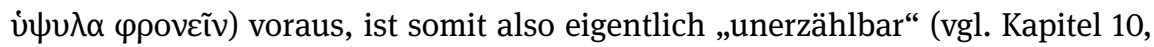
Abschnitt 2).

Vor diesem Hintergrund ist es äußerst bemerkenswert, dass die abschließende Folgerung in V. 24 - dass „diese“ natürlichen (Zweige) nämlich umso mehr in ihren eigenen Baum wieder eingepropft würden - als Bedingungssatz das Protonarrativ aus V. 17 übernimmt, aber der Handlung eben noch eine weitere Episode hinzufügt. Auch wenn in diesem Text daher keine explizite Erzählung vorliegt, ist die Kategorie des Narrativen aus dem Abschnitt nicht fortzudenken: Röm 11,18-23 kann vielmehr mit gutem Recht als eine Interpretation und kritische Besprechung des in V. 17 angesprochenen Protonarrativs gelten. Sofern man, so gibt Paulus hier zu bedenken, diese Ereignisse überhaupt zu einer Erzählung verketten möchte, zeigt eine sorgfältige Analyse der sinnhaften Verbindungen, dass der Plot erst mit der Folgerung in V. 24 vollständig ist.

\subsubsection{Futur in der Apodosis und Protasis}

Ein Sonderfall der für die Protonarrativ-Analyse bedeutsamen indefiniten Konditionalperioden liegt dann vor, wenn nicht nur die in der Apodosis ins Auge gefasste Konsequenz in der Zukunft liegt, sondern sich auch bereits die in der Protasis angesprochene Bedingung auf ein futurisches Geschehen bezieht.

Im Römerbrief ist dies jedoch an keiner Stelle der Fall, wobei natürlich immer wieder ein Sachverhalt ins Auge gefasst ist, der sich auch noch in die Zukunft erstreckt (z. B. in Röm 8,13). Außerdem legt sich für die verblose Konditionalperiode in Röm 8,31 aufgrund der Folgeverse zumindest eine eschatologische Ausrichtung nahe. ${ }^{504}$

Belegt ist der Gebrauch für Paulus jedoch aufgrund von 1. Kor 3,14-15: Hier wird die in V. 13 angekündigte „Feuerprobe“ expliziert, indem zwei mögliche zukünftige Ereignisse genannt werden und der jeweils damit zusammenhängende potenzielle Plot durch die Apodosis fortgeführt wird: „Wenn jemandes

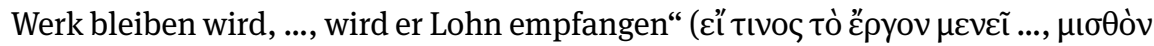
$\lambda \eta ́ \mu \psi \varepsilon \tau \alpha$ ı). Und: „Wenn jemandes Werk verbrennen wird, wird er Schaden leiden,

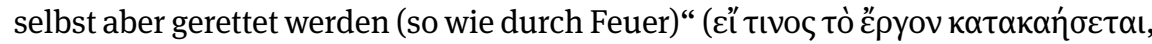

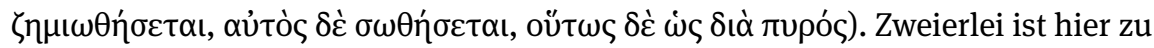
beachten:

Zum einen ist auffällig, dass Paulus in V. 14 eben nicht einfach zu einer Gegenüberstellung mit oí $\mu \varepsilon ́ v$... oi $\delta \varepsilon ́$ (,Die einen ..., die anderen ...“) übergeht, sondern

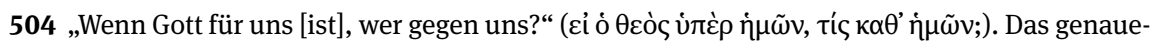

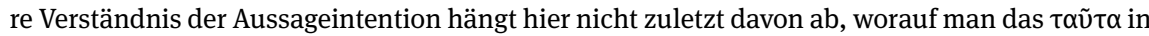

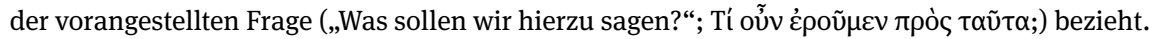


zwei alternative futurische Bedingungen beschreibt, deren Erfüllung von der Grammatik her offengelassen wird. Der Grund hierfür liegt darin, dass die Erfüllung der jeweiligen zukünftigen Bedingung wiederum davon abhängig ist, was der „Jemand“ auf den „Grund“ (welcher Christus sein muss; siehe V. 11) in der

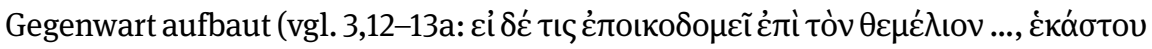

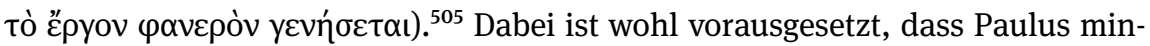
destens für sich selbst ein entsprechend positives Schicksal erwartet (vgl. dann auch explizit in 2. Tim 4,8). Das Protonarrativ des in V. 13 angesprochenen Tages besteht also wohl aus einem durchaus vorausgesetzten Handlungsstrang des Bestehens im Feuergericht und einem lediglich potenziellen Handlungsstrang, der vom gegenwärtigen Handeln abhängig ein alternatives Schicksal skizziert.

Zum anderen muss festgehalten werden, dass es die Formulierung mit $\tau$ Iऽ Paulus erlaubt, diese Skizze des möglichen Handlungsverlaufs auch dem individuellen Leser ${ }^{506}$ für die Ausgestaltung des eigenen eschatologischen Protonarrativs zu übernehmen. Paulus erzählt also nicht einfach prädiktiv über das, was an jenem Tag aus V. 13 geschehen wird (als wäre dies das „Thema,“ siehe hierzu Kapitel 3, Abschnitt 5.3), sondern liefert für diesen temporalen Bezugspunkt verschiedene Ausgestaltungsmöglichkeiten der individuellen Schicksale der Angesprochenen, die durch die Schilderung dazu angehalten werden, ein je individuelles Protonarrativ zu entwerfen. ${ }^{507}$

505 Schrage, Brief I, 300 erklärt leider nicht, wie er zu der Einschätzung kommt, die Konstruktion in V. 12 sei „nicht eindeutig“ und könne auch als indirekter Fragesatz aufgefasst werden. Als indirekter Fragesatz mit $\varepsilon i$ (siehe zu dieser Kategorie ausführlicher oben, Abschnitt 2.1) würde der Konstruktion in der Regel die Satzgliedfunktion des Subjekts oder Objekts zukommen

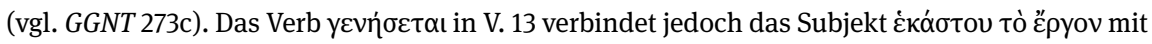
der Subjektsartergänzung $\varphi \alpha v \varepsilon \rho o ́ v$, sodass es nicht möglich ist, den عi-Satz als Subjekt aufzufas-

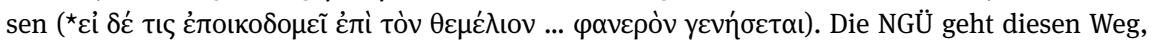
kann dafür aber dann natürlich nichts mit غ́kóotov tò हैpyov anfangen: „Wie nun aber jemand darauf weiterbaut ..., das wird nicht verborgen bleiben. “ Im Hintergrund steht wohl der Versuch von Menge, der allerdings - eher vertretbar - eine Ellipse des notwendigen übergeordneten Verbs zum indirekten Fragesatz annimmt.

506 Oft wird das Weiterbauen in V. 10 mit anderen Lehrern verbunden und explizit vom Beitrag des Individuums in der Gemeinde abgehoben (so etwa Schrage, Brief I, 297). Die Fortführung der Baumetaphorik in 3,16-17 könnte diese Interpretation unterstützen, denn dort wird zwischen der Gemeinde als dem Tempel Gottes und einem тıৎ, der (ebenfalls in einem indefiniten Konditio-

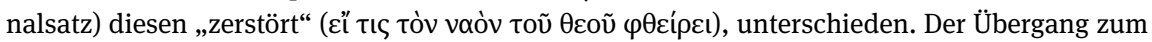
allgemeinen ,,jemand ... unter euch“ ( $\tau$ เ ... Év ن́fĩv) ist jedoch sehr organisch und die Ausbildung individueller Protonarrative auf der Grundlage von 1. Kor 3,11-14 ist damit auf jeden Fall eine sehr plausible Bewirkungsfunktion. Vgl. zur Terminologie oben, Kapitel 9, Abschnitt 4.

$507 \mathrm{Vgl}$. für eine ähnliche Offenheit auch die Kommentare zu Röm 6 in diesem Kapitel, Abschnitt 4.2.2. 
Ein syntaktisch vergleichbarer Fall liegt im corpus Paulinum nur noch in 2. Tim 2,12b vor (wobei Konstruktionen wie 1. Kor 6,2 natürlich inhaltlich nicht weit entfernt sind). ${ }^{508}$ Hier folgen auf die Aussage „Zuverlässig ist das Wort“

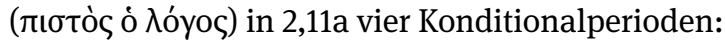

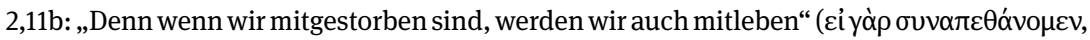

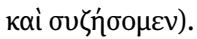

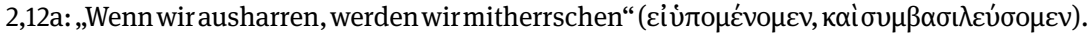

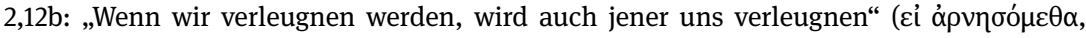

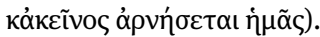

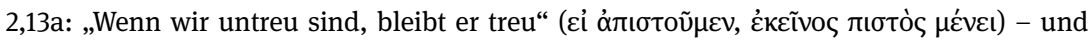

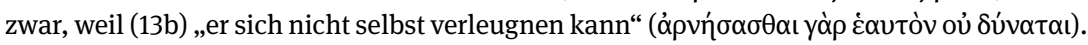

Bereits auf den ersten Blick ist offenkundig, dass hier Konditionalsätze verschiedene Sachverhalte zu unterschiedlichen Zeitpunkten thematisieren: ${ }^{509}$ Bezug auf die Vergangenheit liegt durch den Verweis auf das (potenzielle) Ereignis des

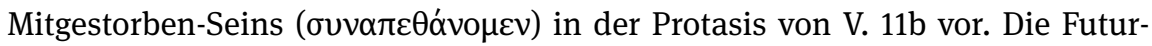
form in der Apodosis kann sich entweder auf das noch ausstehende Leben als Christ oder das eschatologische Leben beziehen..$^{510}$ Im ersteren Fall würde das Präsens der Protasis in V. 12a aufgreifen: Es ginge dann um ein Ausharren im Leben „mit jenem.“ ${ }^{\text {511 }}$ Das daraus folgende, futurisch formulierte Mitherrschen ist zweifellos eschatologisch zu verstehen und markiert den Kontrast zum mühevollen Leben, welches - so die Implikation der Protasis - Standhaftigkeit erfordert.

In V. 11b-12a läge somit ein Protonarrativ vor, welches Vergangenheit, Gegenwart und Zukunft umschließen würde, wobei jedes Element für sich indefinit bliebe, der Zusammenhang innerhalb der Perioden jedoch als notwendig betont wäre. Der Umstand, dass die Protasis von V. 11b kontextuell als erfüllt betrachtet werden kann sowie die auffällige Wiederaufnahme der Apodosis von V. 11b durch

508 Die Protasis von 1. Kor 6,2 steht im Präsens („Wenn durch euch/vor euch die Welt gerichtet

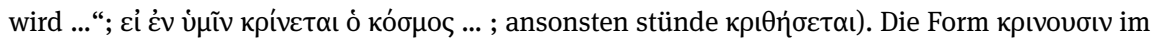
selben Vers, die auf „die Heiligen“ bezogen ist, ist aber wohl futurisch zu akzentuieren. Vgl. auch

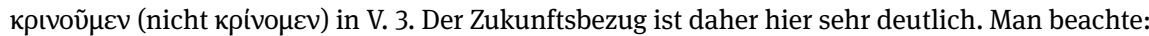
Anders als in 1. Kor 3,14-15 und 2. Tim 2,12 ist die Apodosis hier auf gegenwärtiges Geschehen bezogen. Aus einem zukünftigen Umstand ergibt sich also eine Implikation für die Gegenwart.

509 Erstaunlich ist daher, dass nicht wenige Kommentare den Abschnitt ohne große Berücksichtigung der implizierten narrativen Struktur untersuchen und hierin mehr eine Ansammlung verschiedener „Aussagen“ sehen.

510 Offen gelassen etwa von Marshall, Epistles, 740.

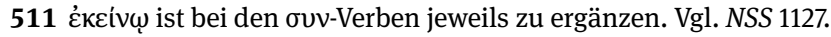


die Protasis von V. 12a zeigen an, dass hier - im Ausharren im Leben mit Christus - der entscheidende Handlungsknoten des Protonarrativs liegt.

Wie knüpft nun V. 12b an diese lineare Abfolge an? In der Protasis begegnet hier - wie in 1. Kor 3,14-15 - nun eine Futurform. Diese kann entweder auf das Endgericht bezogen werden, sodass der Text vom anhaltenden eschatologischen Mitherrschen nun wieder zeitlich einen Schritt zurück machen und eine weiter mögliche Abzweigung der Handlung thematisieren würde. Dann wäre das Verhältnis zwischen V. 12b und V. 13 so zu charakterisieren, dass im letzteren Fall die bei irdischen Fehlern zur Wirkung kommende Nachsicht von ,jenem“ betont würde, im erstgenannten - verwandten, aber eben schlimmeren - Fall jedoch eine fatale Konsequenz folgen würde. ${ }^{512}$

Plausibler ist jedoch die folgende Auflösung: Während V. 12a präsentisch vom Ausharren spricht, nimmt V. 12b futurisch auf die noch ausstehende Möglichkeit des offiziellen Verleugnens Bezug - die Apodosis könnte dennoch auf das Gericht bezogen sein. Versteht man das Verleugnetwerden als ein Ausschluss vom Mitherrschen aus der Apodosis von V. 12a, dann kann man die Periode in V. 12b auffassen als eine Explikation des Zusammenhangs der Periode in V. 12a: Das zukünftige Mitherrschen gilt genau dann, wenn ${ }^{513}$ auch das Ausharren gegeben ist. Nicht erfüllt ist diese Bedingung im Falle des Verleugnens und entsprechend entfällt dann auch die Apodosis aus V. 12a, weil sich die Apodosis von V. 12b dazwischenschiebt.

Das Mitherrschen aus der Apodosis von V. 12b ist folglich abhängig von einem Ausharren, welches darin seinen konkreten Ausdruck findet, dass der Herr unter Druck nicht verleugnet wird. Man könnte an dieser Stelle auch einen prospektiven Konditionalsatz erwarten, doch verzichtet der Autor wohl bewusst darauf, um den Wirklichkeitsbezug dieser Bedingung völlig offen zu lassen.

V. 13 liefert dann nicht nur einen Kontrast zwischen der Möglichkeit „unserer“ Untreue und „,seiner“ Treue, sondern - in 13b - auch einen Kontrast zur Apodosis von V.12b: Während er unter bestimmten Voraussetzungen uns verleugnen wird, ist es ihm unmöglich, sich selbst zu verleugnen.

Von manchen Auslegern wird V. 13 so verstanden, als würde hiermit der logische Zusammenhang zwischen Protasis und Apodosis von V. 12b erläutert: Weil Christus sich nicht selbst verleugnen kann, bleibt er seiner Bereitschaft zum Gericht über die Treulosen treu. Dagegen spricht jedoch die asyndetische Anbindung (yó $\rho$ wäre sonst zumindest zu erwarten) von V. 13a an V. 12b.

512 Siehe zur Diskussion Marshall, Epistles, 740.

513 D. h., die Periode ist (nicht durch die Grammatik vorgegeben, sondern von den Sachverhalten her erschlossen) „bikonditional“ - anders als die Fälle in GGNT 331c. 
Es ist also vielmehr davon auszugehen, dass die Protasis von V. 13a einen gewissen Kontrast zur Protasis von V. 12b bildet und im ersten Fall (,wenn wir verleugnen werden“) der konkrete Akt des Verleugnens im Blick ist, im zweiten Fall (,wenn wir untreu sind“) derjenige (bloßer) ethischer Ungenügsamkeit. Die Apodosis bringt dann also tatsächlich die Treue gegenüber den Untreuen (nicht die Treue gegenüber dem Beschluss zum Gericht) zum Ausdruck.

Das bedeutet, dass die Protasis von V. 13a einen Sonderfall des Ausharrens aus V. 12a thematisiert, quasi die Grenzen dessen auslotet, was noch hier zugerechnet werden kann, während die Protasis von V. 12b außerhalb dieses potenziellen Handlungsstranges liegt, der (noch) zum Mitherrschen führt.

V. 13b bringt zum Ausdruck, dass ein Handlungsstrang nicht vom „bloßen“ Untreusein - also innerhalb des breiten als „Verharren“ gekennzeichneten Geschehens - zum fatalen Ereignis des Verleugnetwerden durch den Herrn führen kann (und somit zum Ausschluss vom Mitherrschen), da dieser dazu aufhören müsste, uns treu zu bleiben - was wiederum das nicht mögliche Ereignis voraussetzen würde, dass er sich selbst verleugnete. Dieser Handlungsstrang wird folglich diserzählt.

\subsubsection{Prospektive Konditionalsätze mit żóv}

\subsubsection{Hinführung}

Auch der durch ćóv + Konjunktiv gekennzeichnete (II) prospektive Fall des Bedingungssatzes („Eventualis“) erscheint im Römerbrief einige Male. Anders als beim indefiniten Konditionalsatz, gilt für die Protasis der prospektiven Konditionalperiode, dass sie etwas bezeichnet, „womit man - unter Umständen - rechnen kann oder muss. “514

Insofern der generell-prospektive Spezialfall „eine verallgemeinernde Aussage, die nicht nur für die Gegenwart gilt, sondern auch, ja sogar vorwiegend, für die Zukunft“ enthält, ${ }^{515}$ sind hier natürlich auch diejenigen Apodosis-Sätze von Relevanz, die keinen Indikativ Futur enthalten.

So ist in Röm 7,5 das Losgemachtsein der Frau von ihrem Mann im Falle

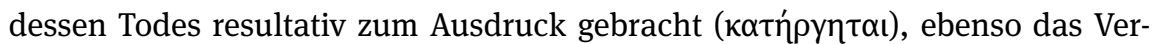

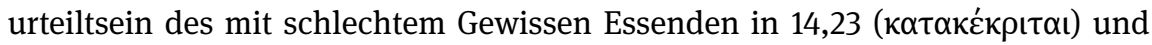
das Gewordensein ( $\gamma \varepsilon ́$ yovev) der Beschneidung zur Unbeschnittenheit im Fall der Gesetzesübertretung in 2,25. 
Typisch ist besonders die Wahl des Präsens wie etwa in Röm 2,25, wo auf

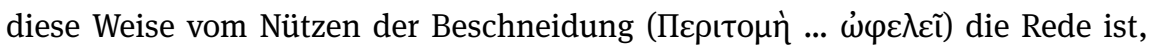
welches an das Halten des Gesetzes geknüpft ist. Ebenso ist auch das „dem Herrn Leben/Sterben/Sein“ in 14,8 präsentisch zum Ausdruck gebracht. Hier ist die Bedeutung des generell-prospektiven Konditionalsatzes für die Rekonstruktion von Protonarrativen besonders augenfällig: Durch die erste Person Singular unterstützt, wird hier auch der Tod eines jeden Lesers mitthematisiert.

Dass der generell-prospektive Konditionalsatz einem Temporalsatz im Sinn von „immer wenn ...“ nahe kommen kann, ${ }^{516}$ zeigt Röm 12,20, wo Prov 25,21-22 zitiert wird, mustergültig: Die hier nahegelegte Verhaltensweise gilt, wann immer ein Feind in eine Notlage gerät.

Sehr interessant für die narratologische Analyse sind aber auch insbesondere diejenigen Fälle, in denen in der Apodosis der Indikativ Futur steht, welcher nämlich in der Regel auf einen speziell-prospektiven Fall hinweist, also darauf, dass die Protasis ,speziell etwas Zukünftiges [bezeichnet], mit dem man rechnen kann oder muss. ${ }^{517}$

\subsubsection{Futur in der Apodosis im Römerbrief}

Im Römerbrief ist diese - auch generell mit Vorsicht anzuwendende $\mathrm{e}^{518}$ - Regel jedoch öfters nicht zutreffend. Untypisch ist etwa die nach der eindeutig generell-prospektiven Konditionalperiode in 2,25 getroffene Wahl des Futurs

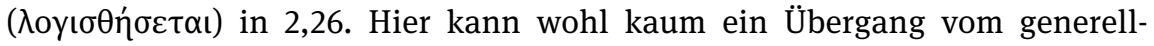
prospektiven zum speziell-prospektiven Fall angenommen werden, zumal in 2,25 mit der zweiten Person Singular ein spezifischerer Bezug vorgenommen zu sein scheint als durch die metonyme Rede von der Beschneidung/Vorhaut in $2,26 .{ }^{519}$

Ebenso wird man kaum aus dem futurischen „Genanntwerden“ ( $\chi \rho \eta \mu \alpha \tau i ́ \sigma \varepsilon$ ) in 7,3 eine spezifische zukünftige Situation ins Auge fassen dürfen. Nicht nur scheint hier kein Unterschied zum Resultativ in 7,2 beabsichtigt zu sein, direkt im Anschluss in 7,3 wird zudem der umgekehrte Fall erläutert, dass der Ehemann stirbt - woran sich dann wieder typisch im Präsens anschließt: „... (so) ist sie frei

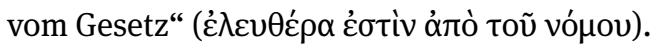

516 Siehe dazu GGNT 282b.

517 GGNT 280c.

518 Siehe die Warnung bei GGNT 282b.

519 GGNT 282d verweist auf Joh 6,51, 7,17 und Mt 15,14 als Beispiele für diese Konstellation. 
Ein wenig anders gelagert ist der Fall in Röm 10,9, wo mit ötı der Grund für das in 10,8 behauptete Nahsein des Wortes eingeleitet wird. ${ }^{520}$ Die Ankündigung

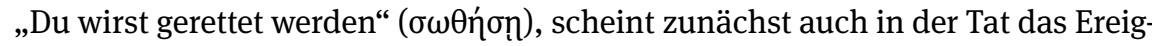
nis einer individuellen Heilszuteilung im Blick zu haben. Dazu passt, dass in der Exegese immer wieder erwogen wurde, ob hier auf eine Bekenntnis- und/oder Akklamationsformel im Zusammenhang mit der Taufe angespielt wird. ${ }^{521}$ Allerdings ist die Wahl der zweiten Person Singular auch durch deren Erscheinen im Zitat aus Dtn 30,14 im Vers zuvor begründet. Hätte Paulus tatsächlich auf das Heil des Einzelnen fokussieren wollen, wäre in diesem Fall wohl sogar der indefinite Konditionalsatz mit عi naheliegender gewesen, weil es bei diesem möglich ist, explizit Vergangenheitsbezug auszudrücken. ${ }^{522}$ Eine klare Zuordnung zu einem spezifisch-prospektiven Fall liegt also auch hier nicht vor, zumal der Wechsel in das Passiv in 10,10 und insbesondere das universelle Zitat aus Jes 28,16 (,Jeder, der an ihn glaubt, wird nicht zuschanden werden“; siehe dazu oben, Kapitel 13, Abschnitt 2.2.4), eine weitere Perspektive einnimmt. Andererseits ist wiederum die Wahl eines Konditionalsatzes im Allgemeinen (etwa gegenüber einem die Aktion weniger betonenden konditionalen Relativsatz; siehe dazu unten, Abschnitt 5.2.3.3) für den „confessional character“ der Passage eine sehr nachvollziehbare Wahl. ${ }^{523}$

Der einzige eindeutig spezifisch-prospektive Konditionalsatz liegt daher in Röm 15,24 vor, also in der oben (Kapitel 11, Abschnitt 8) bereits besprochenen Passage über die Reisepläne. Paulus „hofft“ ( $\dot{\lambda} \lambda \pi i \zeta \omega)$ dort, „auf der Durchreise“ (

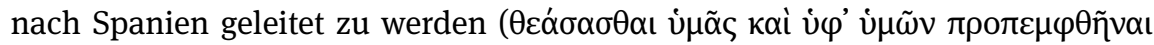

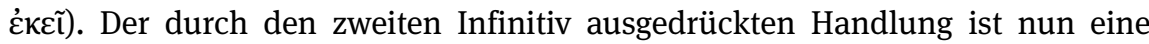
Bedingung zur Seite gestellt: „Wenn ich euch zuerst einigermaßen genossen

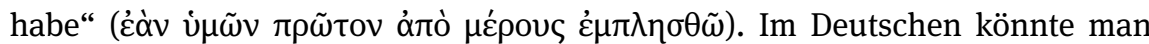
durchaus versucht sein, hier futurisch mit „,... wenn [temporal, d. h.: nachdem] ... ich euch zuerst einigermaßen genossen haben werde“ $\mathrm{zu}$ übersetzen. Die Nähe zum Temporalsatz mit ö $\tau \alpha v^{524}$ ist hier wieder besonders deutlich.

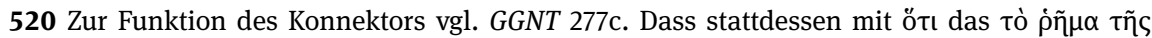

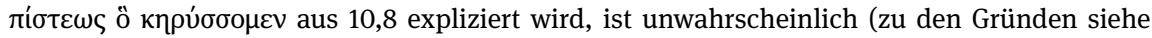
Cranfield, Epistle II, 526).

521 Vgl. etwa Wilckens, Brief II, 227.

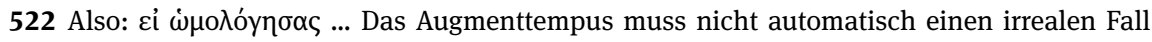
signalisieren. Zur Form vgl. im NT 1. Tim 6,12.

523 Vgl. Caragounis, Development, 199.

524 Siehe dazu oben, Kapitel 7, Abschnitt 4.2.2. 


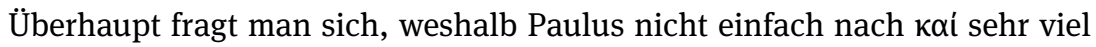
unkomplizierter ein temporal gebrauchtes Partizip eingefügt hat. Schlier ist daher sicherlich zuzustimmen, dass dieser Nachtrag ,etwas verklausuliert“ hinzugefügt ist. ${ }^{525}$ Aus der Formulierung ${ }^{526}$ jedoch „deutlich die Vorsicht dessen ..., der sich dieser römischen Gemeinde gegenüber unsicher fühlt“527 abzuleiten, scheint genau in die falsche Richtung zu weisen: Der Aufenthalt in Rom selbst, die Gemeinschaft mit den noch fremden Geschwistern, wird hier durch die Form des Bedingungssatzes vielmehr betont zum Selbstzweck erhoben und als eigenständiger Bestandteil des Plans ergänzt. ${ }^{528}$

Einen weiteren, wenn auch bei weitem nicht so deutlichen, spezifischprospektiven Fall, könnte man zudem in Röm 11,22 erkennen. Denn in diesem Abschnitt ist die imaginäre Konversation mit einem „Du“ als Gegenüber sehr plastisch ausgestaltet, ${ }^{529}$ sodass innerhalb dieser Fiktion tatsächlich ein konkretes zukünftiges Geschehen ins Auge genommen wird. Die Protasis lautet: „Wenn

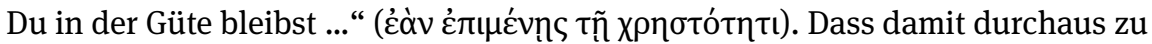
rechnen ist, zeigt bereits die vorausgehende Struktur des Verses, in welchem auf Strenge und Güte Gottes verwiesen wird, wobei letztere dem Du schlicht zuge-

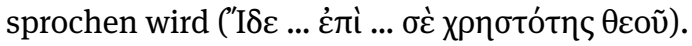

Der nachgeschobene Konditionalsatz qualifiziert somit die gnadenvolle Einstellung Gottes in zweierlei Weise: Zum einen wird natürlich so die zukünftige Erfahrung der Güte an die Bedingung geknüpft, dass der Angesprochene auch seinerseits diesen Gnadenbereich nicht verlässt. Zum anderen aber wird überhaupt erst aus einer die Vergangenheit betreffenden Konstellation ${ }^{530}$ ein auch die Zukunft betreffendes relationales Element: Auch wenn die Apodosis die

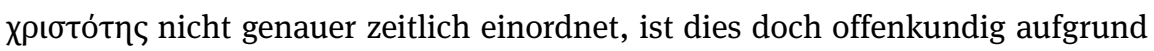
der Fortführung mit દ̇ं $\varepsilon$ í, welches hier im Sinn von „denn sonst“531 erläutert, was im umgekehrten Falle des Nichtbleibens in der Güte für den Angesprochenen folgt: „Auch Du“ - wie schon die natürlichen Zweige in 11,21 - wirst dann

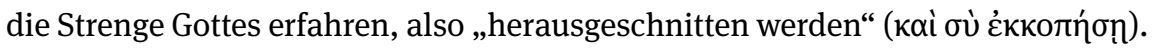

525 Schlier, Römerbrief, 435.

526 Vermutlich bezieht sich Schlier primär vor allem auf die einigermaßen erfolgende Sättigung. 527 Schlier, Römerbrief, 435.

528 So gesehen ist Michel, Brief, 369 - ungeachtet der von ihm angenommenen Hintergründe zuzustimmen, dass das hier bestimmende Motiv das der Höflichkeit ist.

529 Siehe oben, Abschnitt 5.2.1.2 (vgl. auch 3.1.1) zum Hinundher in 11,19-20.

530 Für die „Strenge“ siehe das explizit in der Vergangenheit erzählte Geschehen in 11,22. Die „Güte“ ist nicht auf dieselbe Weise zuvor als Grund des Eingepfropft-Werdens in Erscheinung getreten. Jedoch lässt 11,20 keinen Zweifel daran, dass dies gemeint ist: Das Einpfropfen sei aufgrund des Glaubens geschehen und dies wiederum verbiete jeglichen Hochmut.

531 GGNT 277c. 
Umgekehrt gilt für diese natürlichen Zweige wiederum, dass sie „eingepfropft

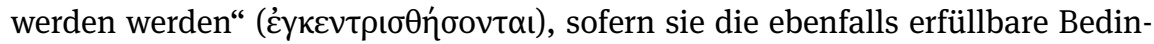

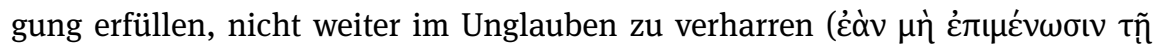

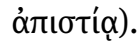

So sehr also das bisherige Geschehen zwei divergierende Handlungsstränge erkennen lässt, ist es Paulus wichtig, darauf hinzuweisen, dass das gegenwärtige Handeln der beteiligten Personen den zukünftigen Verlauf durchaus noch beeinflussen kann. Damit bestätigt sich erneut die Beobachtung, die schon unter Berücksichtigung der indefiniten Konditionalsätze in Röm 11,19-24 gemacht wurden: Paulus interpretiert hier den Plot des Protonarrativs, welches einer der „Eingepfropften“ über seine Aufnahme in den „edlen Olivenbaum“ $(11,24)$ bilden könnte. Es ist somit die richtige Interpretation dieser vergangenen Ereignisse, die $\mathrm{zu}$ einem Verhalten motiviert, welches eine positive Fortsetzung der Erzählung ermöglicht.

Zugleich definiert Paulus hier um, was überhaupt als „positiv“ zu gelten habe: eben nicht das im Kontrast zu den natürlichen Zweigen glücklichere Schicksal. Die Erzählung, die in 11,19 durchschimmert, darf durchaus so aufrechterhalten werden. Doch mit der richtigen Einstellung analysiert $(11,20)$ ermutigt der auf diese Weise erkannte Plot dazu, den Handlungsfortgang für sich selbst bleibend, für die anderen jedoch sich zum Besseren wendend zu erhoffen.

\subsubsection{Futur in der Apodosis in 2. Kor 12,6}

Wie wichtig es für das Textverständnis ist, die verschiedenen Fälle der Konditionalsätze sorgsam auseinanderzuhalten und die entsprechende Auswirkung auf narrative Dynamiken zu beachten, demonstriert das Beispiel 2. Kor 12,6 äußerst eindrücklich. Der prospektive Konditionalsatz „Denn wenn ich rühmen will ...“

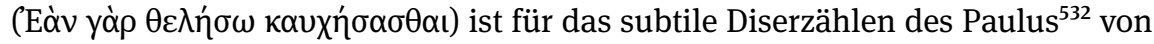
größter Bedeutung.

Der Vers als Ganzes lautet:

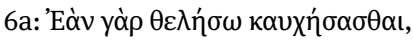

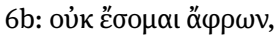

6c: $\dot{\alpha} \lambda \dot{n} \theta \varepsilon ı \alpha \nu$ yà $\rho \dot{\varepsilon} \rho \tilde{\omega}$.

6d: $\varphi \varepsilon^{\prime} \delta$ o $\mu \alpha \mathrm{s} \delta \dot{\varepsilon}$,

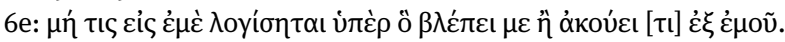

Gerade erst, in V. 5, hatte Paulus noch gesagt, dass er sich nur über den anonymen Mann der Entrückungserzählung 12,1-4 rühmen werde, nicht aber „über

532 Siehe bereits oben, Kapitel 8, Abschnitt 5.5. Vgl. dort auch Abschnitt 5.2.1.2 und 5.2.2.1. 


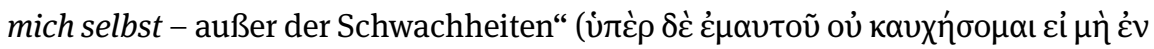

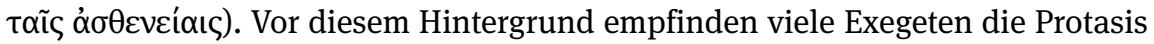
in V. 6a als unangebracht: Wie kann Paulus erst das Rühmen ausschließen und es dann wieder erwägen?

Die Mehrheit löst das Problem durch die Annahme, der Einschub wolle klarstellen, weshalb Paulus auf das Eigenlob verzichte - nicht etwa, weil er damit Unwahres von sich geben würde, sondern, weil er nicht möchte, dass der Eindruck der Korinther auf solchem Lob beruhen würde: „Wenn ich wollte, könnte ich mich sehr wohl auch mit anderen Dingen [d.h. nicht nur mit Schwachheiten] rühmen, ohne mich deshalb zum Narren zu machen; denn was ich sagen würde, währe die Wahrheit. “533

Obwohl diese Position in der Forschung nahezu unangefochten ist, ist sie syntaktisch nicht haltbar. Hätte Paulus diesen Gedanken ausdrücken wollen, hätte ihm eine Konditionalperiode des irrealen Falls zur Verfügung gestan-

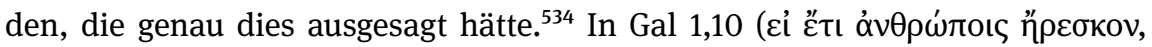

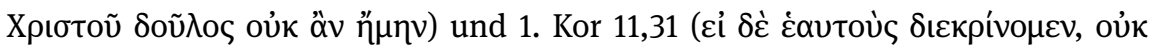

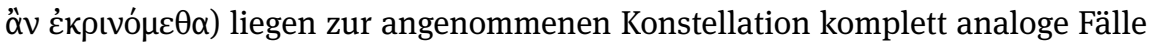
vor: ${ }^{535}$ Im Kontext wird zunächst jeweils ein bestimmter Sachverhalt ausgeschlossen (durch die zu verneinende Frage im ersten Fall und durch 1. Kor 11,29 im

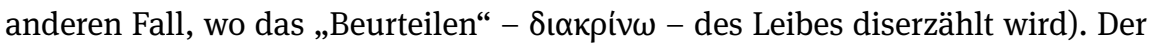
Konditionalsatz nimmt diesen irrealen Fall dann jedoch hypothetisch auf, um die - ebenfalls irreale - Folgerung zu ziehen. Hätte Paulus also sagen wollen, was die allermeisten Exegeten für seine Aussageabsicht halten, so hätte ihm das Griechisch seiner Zeit schlicht keine andere Wahl gelassen, als zu schreiben: Eí

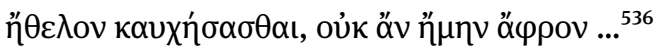

Sucht man im Kontext nach einem Rühmen, das nicht diserzählt wird, könnte man erwägen, ob das Rühmen in V. 6a die Einschränkung aus V. 5 bereits aufnimmt, also gemeint ist: „Für den Fall, dass ich mich meiner Schwachheiten rühme ... “ Die Apodosis in V. 6b würde dann zum Ausdruck bringen, dass solches Rühmen Paulus nicht zum Toren machen würde, da es (V. 6c) der Wahrheit entspräche. Doch weder passt die Anbindung mit yó $\rho$ zu dieser Auslegung (wäre denn nur ein solches Rühmen wahrheitsgetreu und würde Paulus sonst lügen?),

\section{NGÜ.}

534 GGNT 284.

535 Siehe oben, Kapitel 12, Abschnitt 8.3.

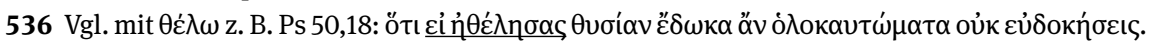

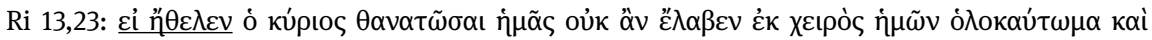

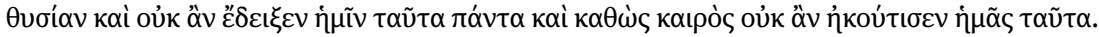


noch scheint diese Interpretation mit dem explizit geäußerten Verzicht auf das Rühmen ( $\varphi \varepsilon^{\prime} \delta$ o $\left.\mu \alpha \iota\right)$ vereinbar zu sein: ${ }^{537}$

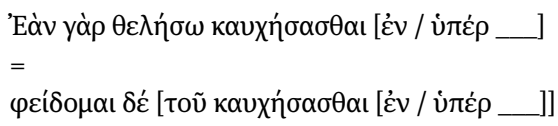

Immerhin hat Paulus in 2. Kor 11,30 ganz explizit das Rühmen für die Schwach-

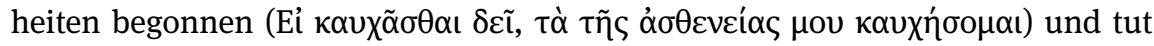
dies zusammenfassend in 12,10 ja auch nochmals.

Es stellt sich also die Frage, welches Rühmen hier einerseits erwogen und andererseits dann doch unterlassen wird. Die einzig kohärente Interpretation ergibt sich, wenn man annimmt, dass der Konditionalsatz überhaupt nicht die zweite Aussage in Vers 5 aufgreift, sondern vielmehr die erste im Blick hat:

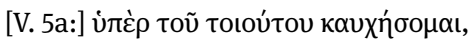

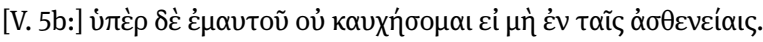

Ein solcher Rückverweis auf V. 5a ist im Kontext äußerst plausibel: Die Verse 2-4 haben eine Geschichte erzählt, die als Grundlage für ein Rühmen dienen könnte, ein eigentlicher Akt des Rühmens scheint demgegenüber aber mit der Schilderung der Ereignisse selbst noch nicht vorzuliegen. Nun, da die Erzählung über das namenlose Individuum zu Ende ist, kündigt Paulus an: „Über einen solchen werde ich rühmen!“ Paulus kommt also der Erwartungshaltung der Korinther nach, indem er einwilligt auch über Visionen und Offenbarungen zu rühmen (V. 1), beharrt aber ironischerweise darauf, nun keine Geschichte über sich zu erzählen, sondern erzählt von einem anderen Christen. Da das Rühmen über andere kein Problem darstellt, kündigt er dann an, sein Rühmen werde nur zu dessen Gunsten ausfallen.

Entsprechend greift der Konditionalsatz nun diese eben angekündigte Bereit-

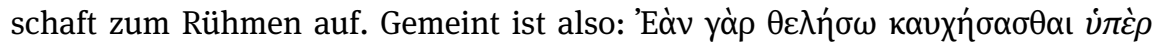

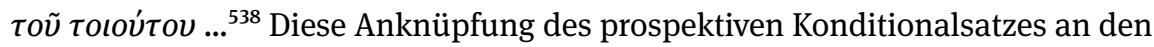
Indikativ des Futurs ist syntaktisch äußerst naheliegend. Es ist erstaunlich, dass dieser Zusammenhang in der Exegese bisher kaum wahrgenommen wurde. Ver-

537 Der Infinitiv ist zu ergänzen. Das von der Wortsemantik her ebenso mögliche „Schonen“ löst das Problem nicht. Zwar kann es absolut, d. h. ohne Genitivobjekt, stehen (2. Kor 13,2), doch der verneinte Finalsatz scheint doch deutlich darauf hinzuweisen, dass hier eine Unterlassung gemeint ist, welche die Leistungen des Paulus vor Augen führen und so den Eindruck seiner Personen dominieren würde.

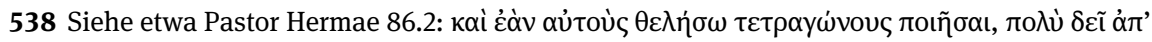

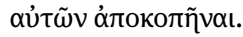


mutlich liegt das daran, dass ein Verständnis des anonymen Mannes in V. 2-4 als Paulus und damit des Paulus' Rühmen über sich selbst für V. 6a vorausgesetzt wird.

Wie oben jedoch gezeigt (Kapitel 8, Abschnitt 5.5), bietet der Text in V. 1-6a zwar aufgrund der Besonderheiten der Narration einige Hinweise auf die Identifikation der Erzählfigur mit dem Erzähler selbst, macht diese jedoch noch nicht explizit. Liest man den Text aus der Perspektive der Erstleser, so verdichten sich im Laufe der Erzählung die Hinweise darauf, dass Paulus hier unzuverlässig erzählt, der von ihm bewusst vermittelte Eindruck, es handle sich um eine von ihm verschiedene Person, also unzutreffend sein könnte (siehe dazu oben, Kapitel 3, Abschnitt 5.4 zum unzuverlässigen Erzählen). Explizit eingeräumt hat Paulus das an dieser Stelle allerdings noch nicht. Er wird dies auch nie tun, sondern vielmehr nur indirekt deutlich machen, dass er der Empfänger dieser Vision ist.

Denn erst in der Erläuterung V. 6e des Unterlassens (V. 6d) wird deutlich, dass die Erzählfigur und der Erzähler ein und dieselbe Person sind: Paulus unterlässt das Rühmen über diese Person, damit die Korinther nicht zu hoch über ihn

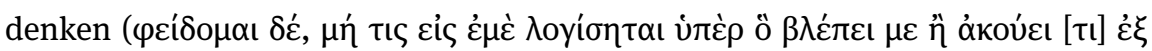
$\dot{\varepsilon} \mu$ õ). Diese Gefahr besteht nun natürlich nur, wenn besagte Person mit ihm identisch sein sollte. Denn das Rühmen einer anderen Person würde wohl kaum als Anlass dafür dienen können, über den diesen rühmenden Paulus zu hoch zu denken. Ohne es also jemals direkt gesagt haben zu müssen, ist jetzt klar, dass das Erzählte dem Erzähler selbst widerfahren ist.

Die hier gebotene Auflösung des Textes löst zudem ein weiteres interpretatorisches Problem. Oft halten Exegeten Paulus vor, er sei an dieser Stelle äußerst inkonsequent: Erst sage er, Ruhm der eigenen Person sei in sich töricht (vgl. etwa 11,16), nun meine er, ein kontrafaktisches Eigenlob würde ihn nicht zum Toren machen, da es ja den Tatsachen entspräche (V. 6c: $\alpha \lambda \eta \dot{\theta} \theta \varepsilon \iota \alpha \nu$ yò $\rho \dot{\varepsilon} \rho \tilde{\omega})$. Wäre, so könnte man fragen, das Rühmen der Super-Apostel also auch nicht töricht, vorausgesetzt, es basierte nicht auf Lügen?

In der hier vorgelegten Interpretation erübrigt sich diese Schwierigkeit vollends: Das Rühmen der eigenen Person stellt die Erfüllung einer hinreichenden Bedingung dar, um als Tor zu gelten. Auch wenn es auf tatsächlich Erlebtes oder Geleistetes zurückgeht, ändert dies folglich nichts. Rühmt man sich jedoch einer anderen Person ist dieses Kriterium nicht erfüllt und es macht entsprechend Sinn, eine weitere notwendige Bedingung $\mathrm{zu}$ erwähnen, welche erfüllt werden muss, damit ein solches Rühmen nicht ebenfalls töricht würde - es muss natürlich immer noch der Wahrheit entsprechen.

Auch wenn Paulus also generell vorhat (V. 5), über die anonyme Person zu rühmen und er (V. 6a) in diesem durchaus zu erwartenden Fall kein Tor wäre (weil 
er eine andere Person rühmen würde und die Wahrheit sagen würde), unterlässt er dies doch zumindest für den Moment (V. 6b).

Dieses Verständnis der Textpassage wird möglich, wenn man nicht nur die Binnenerzählung der Himmelsreise berücksichtigt, sondern auch die durch den Text fragmentarisch ausgedrückte Rahmenerzählung, die auf das Erzählen selbst fokussiert (vgl. Kapitel 8, Abschnitt 5.5), genau verfolgt und die sprachlichen Akzentsetzungen - nicht zuletzt durch den prospektiven Konditionalsatz - ernst nimmt.

\subsubsection{Bedingungen außerhalb von Konditionalsätzen}

\subsubsection{Konditional gebrauchte adverbiale Partizipien}

Auch wenn konditionale Konnexionen in den meisten Fällen durch subordinierende Konnektoren eingeleitet werden, kann derselbe Sinnzusammenhang natürlich noch (III) auf vielfältige andere Weise kommuniziert werden ${ }^{539}-$ im Einzelfall sogar asyndetisch. ${ }^{540}$

Eine durchaus gängige Möglichkeit stellt natürlich die Verbindung einer futurischen Aussage mit einem konditional gebrauchten Partizip dar. ${ }^{541}$ Im corpus Paulinum trifft man einige Male auf diese Verwendungsweise, ${ }^{542}$ jedoch gibt es nur wenige Fälle, in denen durch das Partizip eine Bedingung für ein futurisches Ereignis formuliert wird. ${ }^{543}$

Eine bemerkenswerte Häufung tritt jedoch im 1. Timotheusbrief auf. So ist in

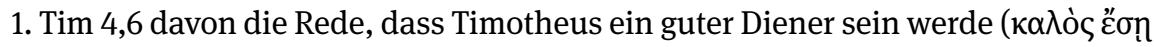

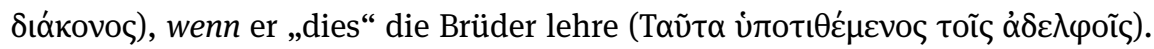
Allerdings ist hier auch ein modales Verständnis („indem“) denkbar. Dieselben Erwägungen gelten für 4,16, wo die Ankündigung „Du wirst auch Dich selbst

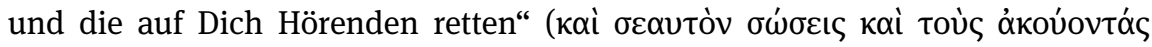
бov), vom anhaltenden Tun des zuvor Aufgetragenen abhängig gemacht wird

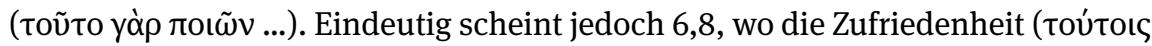

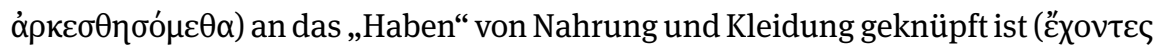

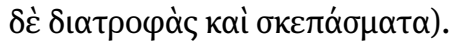

539 Siehe GGNT 331a für eine Übersicht konditionaler Konnektoren.

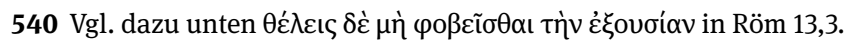

541 Vgl. grundsätzlich GGNT 231h.

542 Z. B. 1 . Kor 6,1.

543 Ein Grenzfall stellt 1. Kor 11,29 dar, wo die Partizipialphrase zu übersetzen ist als: „wenn er

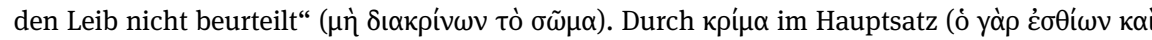

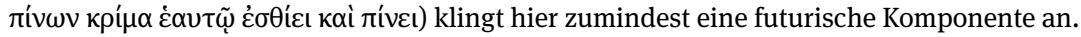


Die einzige Stelle in den unumstrittenen Paulusbriefen, wo ein zukünftiges Ereignis angekündigt wird, jedoch von einer durch ein adverbiales Partizip ausgedrückten Bedingung abhängig gemacht wird, scheint Gal 6,9 zu sein, wo

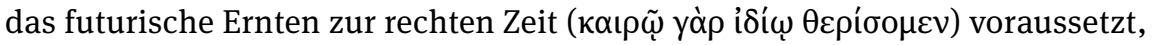
dass etwas nicht geschehen wird: „... wenn wir nicht ermatten (werden)“ ( $\mu$ ì

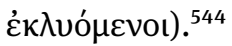

Auch im Römerbrief taucht das konditional gebrauchte Partizip an einigen Stellen auf, aber nicht in Abhängigkeit von einem Verb im Indikativ des Futurs. ${ }^{545}$ Lediglich in Röm 2,27 liegt unter Umständen - d. h. abhängig von der vorgenommenen Interpunktion - eventuell eine futurische Aussage (und kein Fragesatz) vor: „Und es wird dich die der Natur nach Unbeschnittenheit

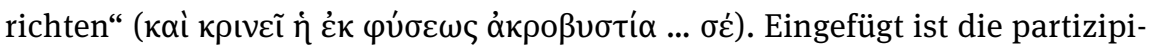

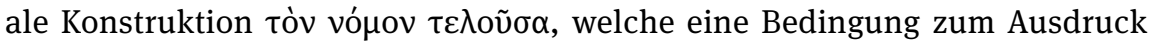
bringen könnte. ${ }^{546}$ Andererseits ist auffällig, dass das korrespondierende „Du“ mit Hilfe eines Partizips attributiv bestimmt wird (,... Dich, der Du ein Übertreter bist“). ${ }^{547}$ Grundsätzlich ist zumindest auch im ersten Fall aufgrund der speziellen syntaktischen Konfiguration, trotz des fehlenden Artikels, ein attributives Verständnis denkbar. ${ }^{548}$

544 Jensen, „Galatians 6:9,“ 102 übersetzt stattdessen: „Let us not grow weary in doing good, because at the proper time we will harvest without growing weary." Er geht davon aus, dass das Partizip nicht konditional aufzufassen sei, sondern vielmehr expliziere, auf welche Weise

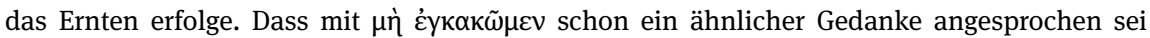
(S. 101-102) erstaunt allerdings wenig: Es entspricht durchaus dem Vorgehen des Paulus, dass er zunächst einen Handlungsstrang entwirft und diesen dann genauer bewertet (siehe für zahlreiche Beispiele oben, Kapitel 12 zum Diserzählen). Entgegen der Versicherung auf S. 100 scheint auch in 1. Kor 11,29 im durativen Partizip (ò yà

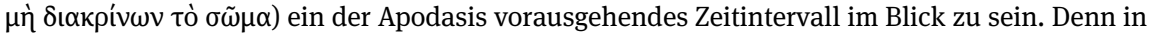

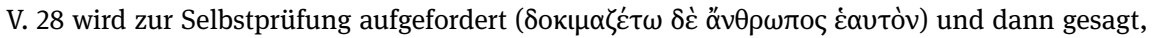

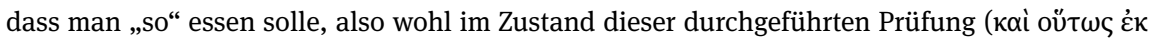

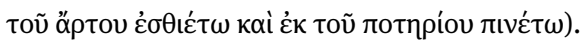

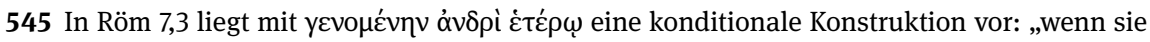

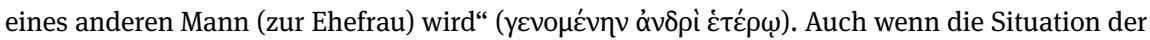
Frau in dieser Passage teilweise mit futurischen Verben beschrieben wird, ist hier die Bezugsgrö-

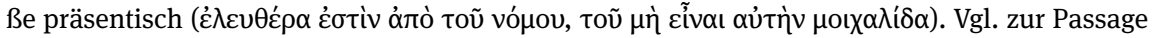
oben, 5.2.2.3.

546 Vgl. Wallace, Grammar, 633.

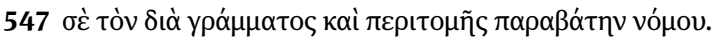

548 Burton 427: ,... but when the participle is limited by an adverbial phrase, this phrase may stand between the article and the noun, and the participle without the article follow the noun.“ Vgl. GGNT 136a. 


\subsubsection{Generell gebrauchte substantivierte Partizipien}

Insgesamt stellt das Partizip dennoch eine bedeutsame Rolle bei der Rekonstruktion von Protonarrativen dar, da beim generell gebrauchten substantivierten Partizip ${ }^{549}$ die Referenz auf eine tatsächlich existierende Größe nicht unbedingt gegeben sein muss. Heinrich von Siebenthal verweist auf das Beispiel von Lk

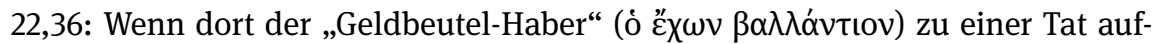
gefordert wird, dann ist damit gemeint: „Wenn einer einen Geldbeutel hat ... “550

Ein solcher Fall liegt auch in Röm 13,2 vor. ${ }^{551}$ Zunächst wird der sich der staat-

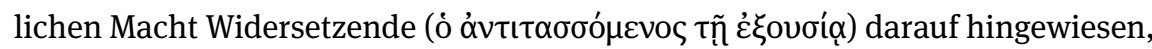

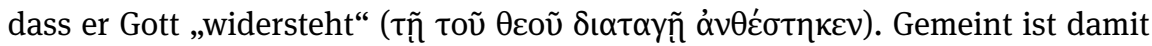
natürlich nicht eine individuelle Einzelperson, ${ }^{552}$ sondern ,jeder, der sich widersetzt." Entsprechend fährt der Text auch fort, indem (nun mit anderem Verb) ${ }^{553}$

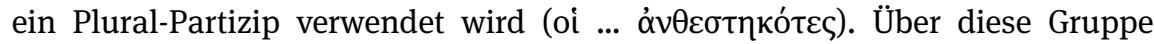

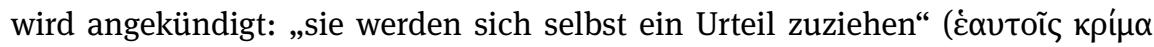
$\lambda \eta \dot{\mu \psi} \psi v \tau \alpha \mathrm{l})$.

Wilckens argumentiert, an dieser Stelle sei keinesfalls an die Strafe durch römische Offizielle, sondern vielmehr an das Endgericht gedacht. ${ }^{554}$ Er begründet das mit den Parallelstellen Mk 12,40/Lk 20,47, 1. Kor 11,29 und Jak 3,1: „Das Futur zeigt durchweg den eschatologischen Aspekt.“"555 Dies übersieht freilich zweierlei: Anders als bei den angegebenen Stellen ist hier im unmittelbaren Kontext von einer gegenwärtigen Machtinstanz die Rede (13,4; vgl. auch noch unmittelbarer

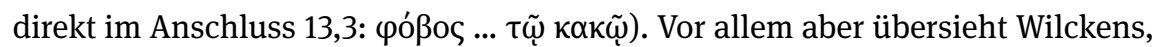
dass die Konstruktion mit dem synonymen крíбıৎ seit Platon (Leg. 767a) belegt ist, bei Plutarch (Mor. 79a) sogar im übertragenen Sinn. ${ }^{556}$

549 Vgl. GGNT 237a.

550 GGNT 331e.

551 Vgl. auch Röm 14,23, wo dem Zweifelnden (

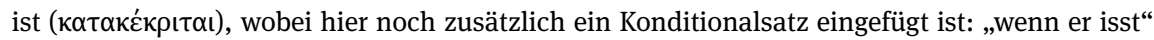

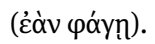

552 Vgl. GGNT 237a.

553 Beide Verben werden von LN 39.1 derselben semantischen Domäne zugeschrieben und erhalten dieselbe Definition (,to oppose someone, involving not only a psychological attitude

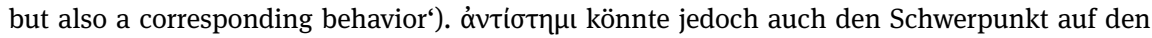
Widerstand gegenüber bestehendem Druck (LN 39.18: ,to resist by actively opposing pressure or power') legen.

554 Wilckens, Brief II, 33.

555 Wilckens, Brief II, 33.

556 Für die Konstruktion mit кpíøıৎ in einem theologischen Kontext siehe wiederum PseudoClementinen 20.4.5. 
Nun ließe sich natürlich argumentieren, dass es auf jeden Fall irgendwelche konkreten Personen gegeben haben dürfte, welche die Bedingungen erfüllt

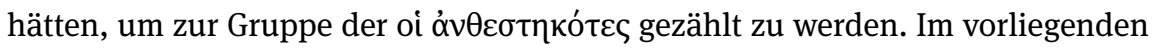
Text folgt die Einführung dieser Gruppe jedoch der expliziten Aufforderung in Röm

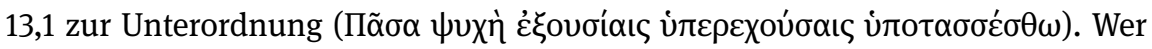

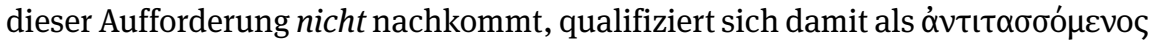
und muss sich entsprechend vor Konsequenzen fürchten.

Röm 13,3b geht denselben Weg dann wieder Rückwärts: „Willst Du Dich

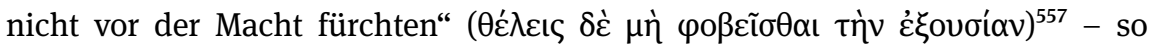
wird das römische Individuum, welches vor der Entscheidung steht, einer der

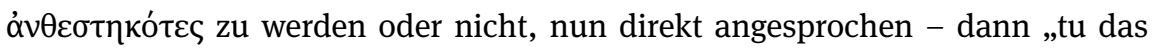

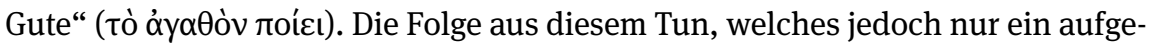
fordertes und damit ein potenzielles ist, wird mit kai apodoticum eingeleitet: ${ }^{558}, \ldots$

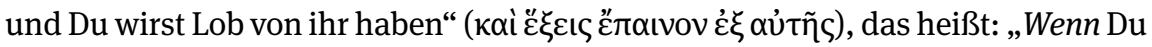
das Gute tust, dann wirst Du Lob von ihr haben.“ Wie das angekündigte „Empfangen“ ist auch dieses versprochene „Haben“ an eine Bedingung geknüpft, deren Umsetzung Paulus durch den Imperativ den Römern nahe legt.

Im folgenden Vers 13,4 wird der eventuelle Fall, dass das „Du“ der Aufforderung nicht nachkommt, mithilfe eines prospektiven Konditionalsatzes durch-

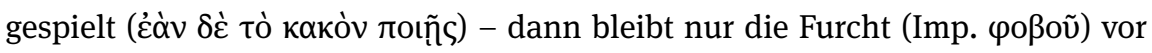
dem kommenden Urteil (13,2: крí $\mu \alpha)$. Das Habenwerden ( $\left.{ }^{2} \xi \xi \iota \varsigma\right)$ ist natürlich kein „eschatologisches Futur,“ da die staatliche Macht als Quelle angegeben ist. Auch

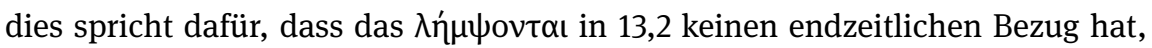
sondern in einer analogen zeitlichen Entfernung liegt, in welcher sich die Folgen der verschiedenen möglichen Handlungen manifestieren werden/würden.

\subsubsection{Relativsätze mit konditionalem Nebensinn}

Es gibt noch weitere Möglichkeiten, im Griechischen ein generelles Subjekt auszudrücken, welches es erlaubt, eine futurische Ankündigung konditional einzuschränken. Dies zeigt etwa ein Blick auf Röm 2,12, wo Paulus die Konsequenz des Textes 2,6-10(/11) (siehe dazu oben, Kapitel 13, Abschnitt 2.2.4) verteidigt. ${ }^{559}$

In diesem Vers liegen zwei futurische Ankündigungen vor: „sie werden auch

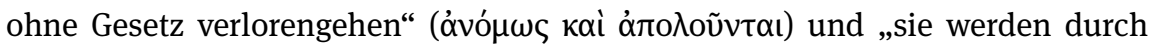

557 Zur asyndetischen konditionalen Konnexion vgl. GGNT 331e.

558 Siehe GGNT 331a zu dieser Möglichkeit. Zum kai consecutivum siehe GGNT 252,29. Solche Verwendungsweisen können dann vorliegen, wenn der Kontext die Art der Konnexion klärt (GGNT 322a). Bei einer Kombination aus Imperativ und Indikativ Futur ist dies der Fall.

559 Vgl. Wilckens, Brief I, 131. 
Gesetz gerichtet werden“ ( $\delta ı \alpha ̀$ vó nicht unter der Überschrift zukunftsgewisser Vorausdeutungen besprochen, da

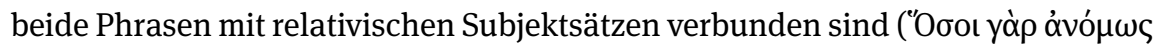

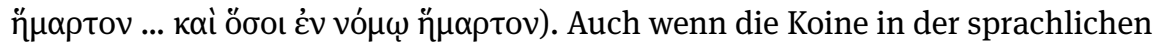
Kennzeichnung von Relativsätzen mit konditionalem Nebensinn nicht mehr so deutlich ist, wie das im klassischen Griechisch der Fall war, ${ }^{560}$ so ist dieser Aspekt hier doch recht eindeutig zu identifizieren. ${ }^{561}$

\subsubsection{Substantive mit lediglich eventueller Referenz}

Interessanterweise stoßen wir direkt im Folgevers auf noch eine weitere Option, mit welcher der konditionale Vorbehalt zum Ausdruck gebracht werden kann. In Röm 2,13 wird der Gedanke fortgeführt durch die Verneinung eines präsentischen Gerechtseins der Hörer des Gesetzes bei Gott.

Dem tritt als Kontrast die zukünftige Rechtfertigung der „Täter“ des Gesetzes

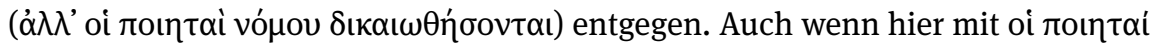
kein Partizip vorliegt, gilt doch, dass die durch das Nomen bezeichnete Gruppe

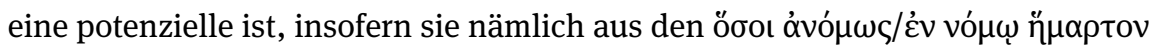
(V. 12) besteht.

\subsection{Beabsichtigte Ereignisse}

\subsubsection{Hinführung}

In der Diskussion der Struktur narrativer Vertextungen wurden Ereignisse, die in der Zweck-Proposition einer finalen Konnexion auftreten, als keine Erzählungen konstituierend eingestuft, da sie bezüglich ihrer Erfüllung innerhalb der Konnexion unbestimmt sind (vgl. Kapitel 5, Abschnitt 3.4). Unter dem Gesichtspunkt impliziter Erzählungen können sie nun jedoch sinnvoll narratologisch erfasst werden. Vorausdeutungen auf die Zukunft des Erzählers stellen sie freilich nur dann dar, wenn das zu erreichende Ziel aus dessen Warte heraus noch aussteht.

Das Spektrum finaler Konnektoren ist sehr groß. ${ }^{562}$ Neben die subordinie-

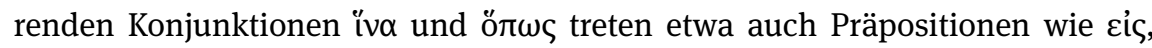

560 Vgl. etwa GGNT 290e: „Wie es durch eine einzige Verfehlung für alle Menschen zur Verurteilung [kam], so [kommt es] auch durch eine einzige Rechtstat für alle Menschen zur lebenswirkenden Gerechtsprechung.“

561 Burton 301.

562 Die Darstellung dieses Absatzes folgt der Übersicht bei GGNT 336a. Siehe auch GGNT 259j für eine Übersicht über Ausdrucksmöglichkeiten von Finalangaben (die jedoch nicht allesamt satzwertig $-d$. h. propositionsbildend und damit relevant für diese Untersuchung - sind). 


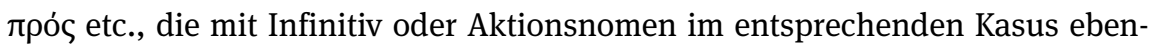
falls eine Ziel-Proposition ausdrücken können. Ebenfalls eine finale Konnexion mit einer anderen Proposition bildet der Infinitiv der Absicht und das final gebrauchte adverbiale Partizip. Dazu tritt das Relativpronomen ős, auf welches Indikativ Futur oder Konjunktiv zur Markierung eines Relativsatzes mit finalem Nebensinn folgt. Der konjunktionale Konnektor ist jedoch mit großem Abstand am häufigsten.

So gibt es etwa bei Paulus keinen eindeutigen Beleg für den Relativsatz mit finalem Nebensinn, ${ }^{563}$ auch wenn ǒs mit Indikativ Futur recht häufig ist. ${ }^{564}$ Einigermaßen naheliegend wäre dieser Gebrauch lediglich im Zitat aus Jes 40,13 in

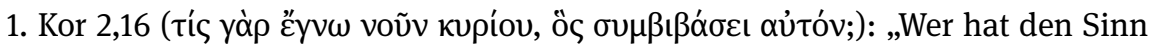
des Herrn erkannt, um ihn zu unterweisen?“ (Wobei eine konsekutive Auflösung mit „dass“ hier angebrachter scheint. $)^{565}$

Zu erwägen wäre er unter Umständen auch für Eph 6,16: Dort wird zum Ergrei-

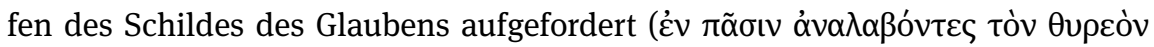

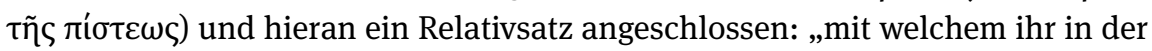
Lage sein werdet, alle feurigen Pfeile des Bösen auszulöschen“ (c่v $\tilde{\dot{\omega}} \delta v v \eta ́ \sigma \varepsilon \sigma \theta \varepsilon$

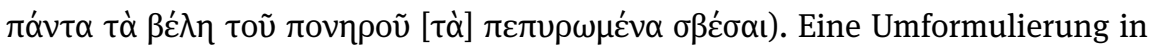

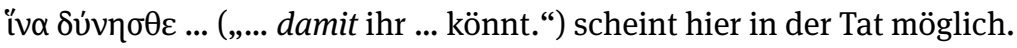

Eine solche Umformulierungsprobe ergibt auch noch guten Sinn in 1. Kor 4,17: Die Sendung des Timotheus wird einerseits durch zurückweisenden $\delta ı \dot{\alpha}$

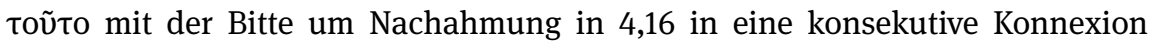

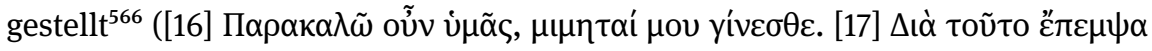

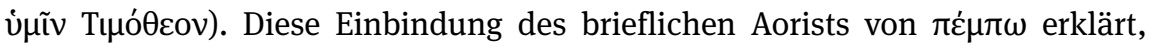
weshalb auf die Aussage hier keine Zweckangabe mit iv $\alpha$ folgt. ${ }^{567}$ Dennoch ist die Weiterführung des Textes in finaler Hinsicht nicht ohne Relevanz: Auf die Beschreibung des Timotheus als „mein geliebtes und treues Kind im Herrn“ (ö

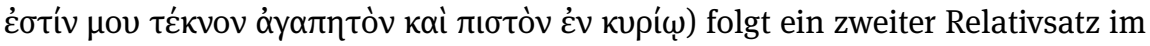
Futur: „... der euch erinnern wird an meine Wege in Christus, wie ich überall in

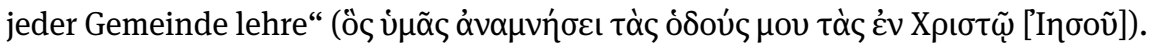

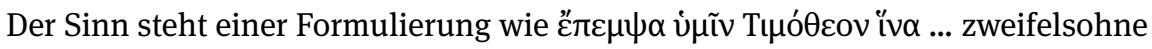
nahe.

563 Röm 2,6, Röm 3,30, 1. Kor 1,8, 1. Kor 4,5, 4,17, 10,13 (2x), 2. Kor 11,15, Phil 3,21, 1. Thess 5,24, 2. Thess 2,8, 2. Thess 3,3,1. Tim 6,15, 2. Tim 4,8.

564 Für andere Relativpronomina siehe Gal 6,16, 2. Thess 1,9 und 2. Tim 2,2.

565 Vgl. GGNT 290d.

566 Zum Konnektor vgl. GGNT 333a. Für die Entscheidung, hier nicht von Kausalität zu sprechen, siehe oben, Kapitel 4, Abschnitt 3.4.

567 Vgl. weiter unten zu diesen Konstruktionen. 
Was den Konjunktiv im Relativsatz angeht, so gebraucht Paulus ihn relativ häufig, um einen konditionalen Nebensinn $\mathrm{zu}$ kommunizieren, ${ }^{568}$ jedoch nie für einen finalen Nebensinn. Was das Zusammenspiel von Relativsätzen und finalen Konnexionen in den Paulusbriefen angeht, fällt vielmehr auf, dass Finalsätze recht häufig an Relativsätze anknüpfen, um gemeinsam eine SacheBeschreibungs-Konnexion zu bilden. Ein Konzept der übergeordneten Konstruktion wird also expliziert, indem es als Mittel für einen bestimmten Zweck charakterisiert wird. Der Relativsatz übernimmt dabei dann die erstgenannte Funktion des Mittels. ${ }^{569}$ Die Häufung in den umstrittenen Paulusbriefen hängt wohl direkt mit den dort oft gebotenen Erklärungen der paulinischen Motivation zusammen. ${ }^{570}$

\subsubsection{Zukunfts-Bezug der Zweck-Proposition}

\subsubsection{Bei präsentischer Mittel-Proposition}

Für die narratologische Analyse ist natürlich von großer Bedeutung, ob sich die Zweck-Proposition tatsächlich auf die Zukunft bezieht (vgl. etwa im Abschnitt zum elliptischen Erzählen - Kapitel 8, Abschnitt 3.6 - zu Gal 1,18, wo ein in der Vergangenheit liegendes und auch erreichtes Ziel ausgedrückt wird).

Der Zukunftsbezug des Finalsatzes ist in der Regel recht unproblematisch, wenn die Mittel-Proposition indikativisch formulierten Gegenwartsbezug aufweist (sofern dabei kein überzeitlicher Präsens-Gebrauch vorliegt). ${ }^{571}$ In der Regel wird in den Paulusbriefen dabei aber lediglich eine gegenwärtige Sprechhandlung des Autors aufgegriffen und auf ihren Zweck hin expliziert (d. h.: „Ich schreibe dies, damit ..." usw.). ${ }^{572}$

568 Vgl. etwa Röm 16,2, 1. Kor 6,18, 11,27, 2. Kor 11,21, Gal 5,10, 5,17 (innerhalb eines ǐva-Satzes

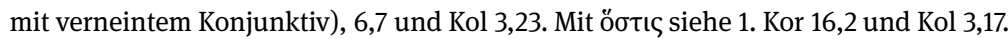

569 So etwa in Gal 2,5, Eph 2,10, 6,22, Phil 3,8, Kol 1,18, 4,8, 1. Tim 1,20, Tit 2,14, Phlm 13. Kol

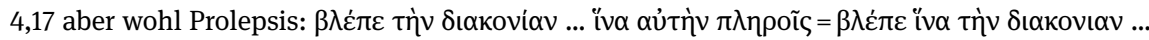
$\pi \lambda$ поĩ (so NSS 1093), d. h.: „Achte darauf, dass ...“ Zu beachten ist ein etwaiger Wechsel des Agens (vgl. Kol 4,8) oder der Diathese (vgl. 1. Tim 1,20).

570 So ja auch in Gal 2,5, Phil 3,8 und Phlm 13. Eine wirklich aussagekräftige stilistische Untersuchung müsste natürlich auch alternative Ausdrucksmöglichkeiten miteinbeziehen, wie etwa die Konstruktion mit attributivem Partizip und anschließendem Finalsatz. So etwa prominent für Christus in Gal 1,4. Zur Stelle vgl. auch im Detail unten, Abschnitt 5.3.2.6.

571 Vgl. etwa Röm 3,19. Die Interpretation ist im Einzelfall nicht ganz unproblematisch. Vgl. etwa 1. Kor 9,12 und 1. Kor 9,23, insbesondere aber auch 2. Tim 2,10. Siehe demgegenüber die explizite Markierung mit ácí (,ständig“) in 2. Kor 4,11.

572 So etwa in 1. Kor 7,35, wo der Zweck durch eine Negativum-POSITIVUM-Verbindung ausgedrückt wird und das positive Ziel durch $\pi$ ó́ mit Infinitiv verwirklicht wird. Vgl. auch 2. Kor 5,12, 13,10, Kol 2,4, 1. Tim 1,18. 
Ergiebiger ist aber etwa 2. Tim 2,10, wo der Autor von seinem Erdulden um

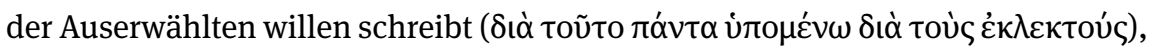
und daran den Zweck anhängt: „... damit auch sie die Rettung, die in Christus

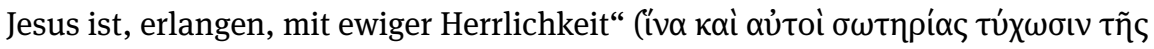

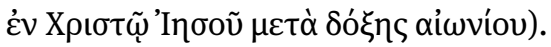

Vergleichbar ist in den unumstrittenen Paulusbriefen etwa 1. Kor 11,32, wo in einem konditionalen Partizip die Möglichkeit aufgegriffen wird, dass „wir vom

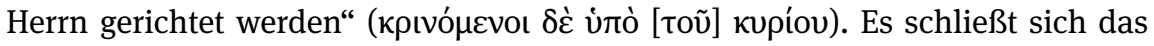
FOLGE-Element dieser konditionalen Konnexion an, welches wiederum aus zwei Propositionen besteht, die zusammen eine finale Konnexion eingehen: Für den

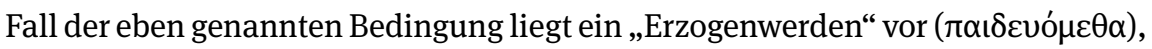

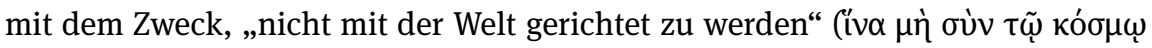
$\kappa \alpha \tau \alpha \kappa \rho \imath \theta \tilde{\omega} \mu \varepsilon v)$. Hier ist es also das (potenzielle) Handeln des Herrn, welches durch Verweis auf das anvisierte futurische (negativ formulierte) Ziel expliziert wird.

Recht häufig ist es auch der Fall, dass im Präsens auf eine Handlung verwiesen wird, welche eine zukünftige Konsequenz hat, an die wiederum ein Finalsatz angefügt ist. In der Regel geht es hierbei um präsentische mentale Zustände (Hoffnungen etc.), die auf zukünftiges Geschehen ausgerichtet sind, welches als Mittel zum Erreichen eines bestimmten Zwecks betrachtet wird. So folgt in Röm 1,11 auf das gegenwärtige Verlangen (દ่лıло $\theta \tilde{\omega})$ der Inhalt des-

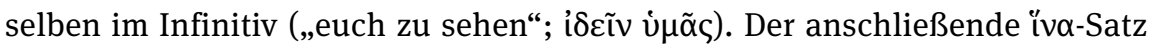
gibt an, mit welchem Zweck dieses zukünftige - gegenwärtig erwünschte Geschehen erfolgen würde: „um euch etwas geistliche Gabe zu vermitteln“

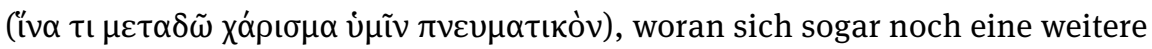
nochmals untergeordnete Finalangabe (diesmal mit Präposition und Infi-

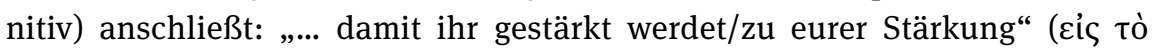

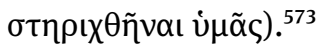

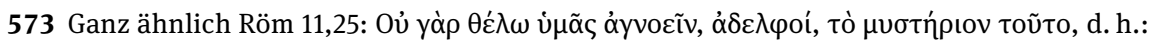
„Denn ich will nicht, dass ihr ... dieses Geheimnis nicht kennt“ = „Denn ich will, dass ihr wisst ...“ (Litotes). Es schließt sich der Zweck an, der durch das Mittel der zu erfolgenden und gegenwärtig gewollten Inkenntnissetzung realisiert werden soll: „damit ihr nicht selbst

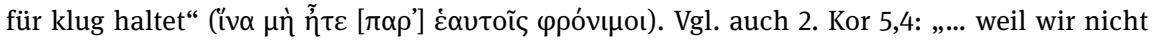
entkleidet, sondern bekleidet werden wollen, damit das Sterbliche verschlungen werde

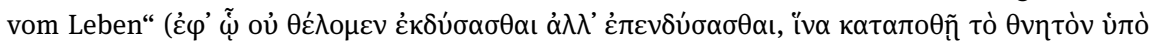
$\tau \tilde{n} \varsigma \zeta \omega \tilde{n} \varsigma)$. Zur Stelle vgl. oben, Abschnitt 4.4.2.1. Ebenso Tit 3,8: „Und ich will, dass Du auf diese Dinge bestehst, damit die an Gott Gläubigen darauf bedacht sind, sich um gute Werke

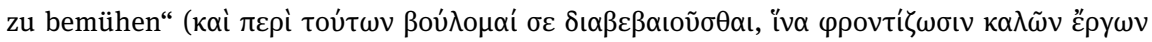

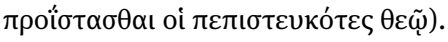




\subsubsection{Bei futurischer Zweck-Proposition}

Noch unproblematischer ist der Zukunftsbezug der Zweck-Proposition freilich dann, wenn bereits das als Mittel gekennzeichnete Geschehen futurisch ist. Mustergültig wird dies von 1. Kor 15,28 demonstriert. Hier liegt ein temporales Gefüge vor, welches zwei sich tangierende Situationen zum Ausdruck bringt (siehe oben, Kapitel 7, Abschnitt 4.6 zur „sobald“-Gleichzeitigkeit): „Sobald ihm aber alles

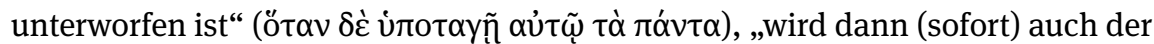

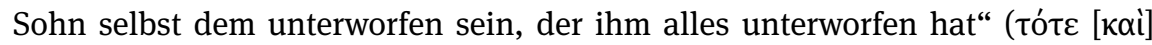

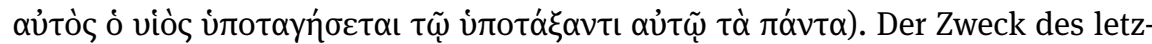
teren Geschehens besteht darin, dass Gott „alles in allem sei“ (ivo ñ o o $\theta$ cò $[\tau \dot{\alpha}]$

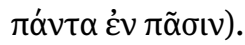

In 1. Kor 16,6 liegt ein syntaktisch vergleichbarer Fall vor, wobei hier kein eschatologisches Geschehen im Blick ist, ${ }^{574}$ sondern ein Reiseplan: „Bei euch

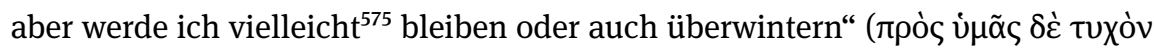

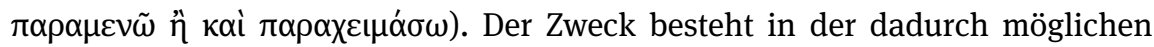
Unterstützung bei der (sich anschließenden) Weiterreise an ein noch unspezifi-

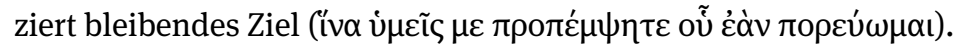

\subsubsection{Im Fall einer Aufforderung in der Mittel-Proposition}

Ein Zweck wird natürlich auch dann als zukünftiges Ziel erkennbar, wenn der entsprechende Finalsatz von einem Imperativ abhängig ist. Dieser Gebrauch ist im corpus Paulinum (mit einer starken Häufung in den Pastoralbriefen) deutlich gebräuchlicher als die eben besprochene Abhängigkeit vom Indikativ des Futurs. ${ }^{576}$ Ein Beispiel im Korintherbrief, wo der Gebrauch mehrfach auftaucht,

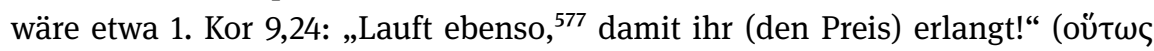

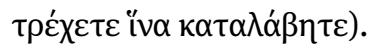

574 Einen weiteren solchen Fall stellt vermutlich auch 2. Kor 11,12 dar, zumindest, wenn man "0

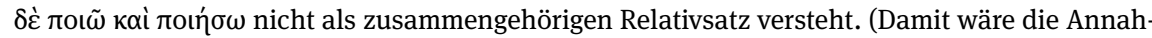
me verbunden, dass der Hauptsatz ausgefallen wäre. Vermutlich wäre ein zeitloses Präsens zu ergänzen.) Wesentlich plausibler ist, dass lediglich “O $\delta \varepsilon \dot{~} \pi 0 เ \tilde{\omega}$ den Relativsatz darstellt: „Was ich

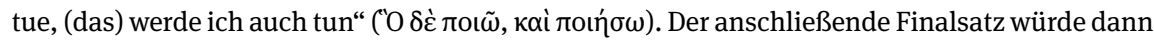
explizieren, welcher Zweck durch das Mittel der Fortführung der Praxis erreicht werden soll:

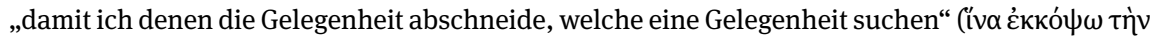

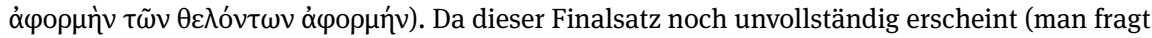
sich: „Eine Gelegenheit wozu?“), ist der sich anschließende ǐvo-Satz wohl am plausibelsten als Ersatz des epexegetischen Infinitivs (siehe f) bei GGNT 272a) zu verstehen.

$575 \mathrm{Zu}$ túxov vgl. oben, Abschnitt 4.3.

576 Siehe 1. Kor 3,18, 5,7, 7,5, 9,24, 11,34, 16,2, 2. Kor 11,16, Eph 4,28.29, 6,13, Phil 1,27, Kol 3,21, 2. Thess 3,14, 1. Tim 4,15, 5,7.16.20, 6,1, Tit 1,13, 3,13.

577 Oder rückverweisend auf die Läufer. Dann wäre ĩv konsekutiv („,So ..., dass ...“). 
Für die Analyse ist zu beachten, dass die Zweck-Angabe recht häufig Äußerungsbezug aufweist: Ebenso wie der kausale Konnektor in Eph 4,25 den Grund dafür anführt, weshalb der Autor die Aufforderung ausspricht, ${ }^{578}$ spezifiziert die Finalangabe nicht immer primär, mit welchem Ziel ein Handeln verfolgt werden soll. Vielmehr kann der Autor dadurch auch seine eigene (Sprech-)Handlung der Aufforderung motivieren. Dabei dürfte diese Zielsetzung in der Regel auch für die Angesprochenen erstrebenswert sein, doch ist dies keinesfalls zwangsläufig der Fall.

Man könnte hier etwa auf 1. Kor 11,34 verweisen: Der Aufforderung im

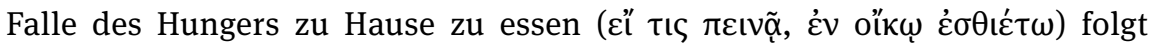

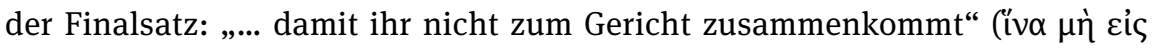

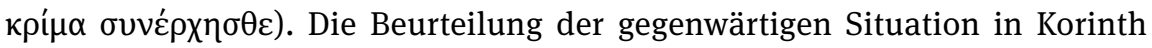
in 11,22 macht deutlich, dass für Paulus das Grundproblem in der Verachtung der Gemeinde Gottes und den beschämenden Umgang mit den Geschwistern

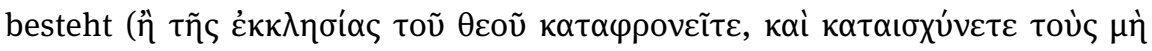

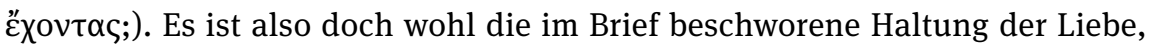
welche dem Individuum zur eigentlichen Motivation gereichen sollte! ${ }^{579}$ Es kann kaum Paulus' Intention sein, das Handeln der Korinther lediglich in ihrer Angst vor dem Gericht zu verankern. ${ }^{580}$

Wenn der aus Sprecherperspektive mit der Aufforderung verbundene Zweck nicht identisch ist mit der Motivation des Handelns der Angesprochenen, so bleibt die Konnexion dennoch final. Es ist also nicht zulässig, İva allein aus diesem Grund als konsekutiven Konnektor zu verstehen. So schreibt etwa Oepke zur Stelle: „Ebenso ist in 11,34 ... die Meinung nicht, die Korinther könnten es auf Gericht abgesehen haben, vielmehr, ihr Verhalten könne die tatsächliche Folge des Gerichts haben. “581 Das von Oepke im Hinblick auf das عiৎ крí $\mu \alpha$ Gesagte, wird von Schrage dann auf die Konjunktion übertragen: „Iv $\alpha$ bezeichnet auch hier ... die tatsächliche Folge. " ${ }^{582}$ Zweifellos ist konsekutives ív $\alpha$ eine nachklassische Entwicklung, die im NT recht häufig anzutreffen ist, ${ }^{583}$ die Kategorie des Äußerungsbezugs liefert jedoch auf jeden Fall eine hinreichende Erklärung für den Gebrauch des finalen ivva in 1. Kor 11,34.

578 Vgl. die Analyse des Beispiels bei GGNT 322d. Siehe zum Äußerungsbezug bei kausalen Konnexionen auch allgemein GGNT 333d.

579 Vgl. grundsätzlich Zimmermann, Logik.

580 Ganz ähnliches gilt freilich auch für die konsekutive Konnexion, die in V. 33 durch $̋ \sigma \tau \varepsilon$ im Verhältnis zum vorher Geschriebenen markiert wird.

581 Oepke, „हiৎ,“ “ TWNT 2:427.

582 Schrage, Brief III, 57.

583 GGNT 272a. 
Recht deutlich ist der Äußerungsbezug auch in der finalen Konnexion in 1. Kor 16,2: Zum wöchentlichen Sammeln wird aufgefordert, „damit nicht, wenn

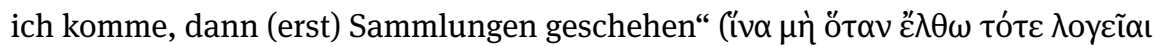

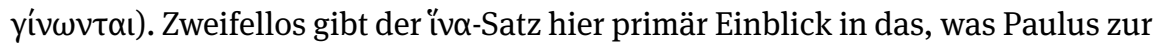
Äußerung der Aufforderung antreibt. ${ }^{584}$

Äußerungsbezug wäre auch für 1. Tim 6,1 und Tit 3,13 zu diskutieren. Absolut eindeutig liegt er in 2. Kor 11,16 vor: Die Korinther werden aufgefordert, Paulus nicht für einen Toren zu halten und - falls sie es doch nicht lassen können -

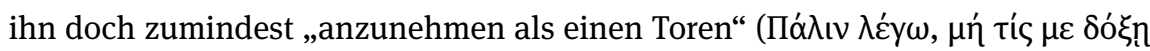

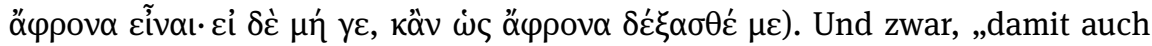

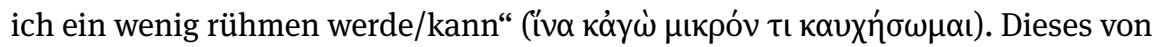
Paulus angestrebte Ziel ist es wohl kaum, welches die Korinther zur Entscheidung für Paulus und gegen die Konkurrenten bewegen wird. Vielmehr ist vom Kontext her klar, dass das bereits erfolgte Rühmen die motivierende Funktion haben soll. (Von einer „Spannung“ zwischen diesen beiden Gedanken muss aber eben auch hier nicht ausgegangen werden, ${ }^{585}$ da der Äußerungsbezug der finalen Konnexion eine absolut zufriedenstellende Erklärung für die Formulierung bietet. Vgl. oben zum „konsekutiven“ “iv $\alpha$ in diesen Fällen.)

Statt des Imperativs kann natürlich auch der adhortative Konjunktiv stehen. Dies ist etwa im Zitat der unterstellten Rede in Röm 3,8 der Fall: „Lasst uns das

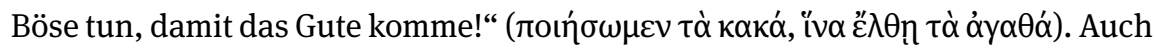
andere Verwendungsweisen des Konjunktivs sind zu beachten, da bei Paulus mit abhängigem ǐva belegt: So etwa in Röm 6,1 nach deliberativem Gebrauch (,Sollen

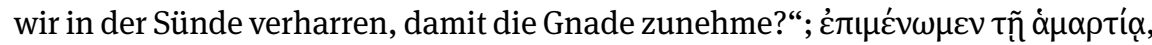

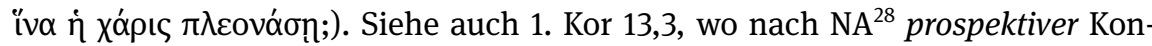
junktiv folgt: „Und falls ich all das Meine zur Speisung der Armen zur Verfügung stelle und wenn ich meinen Leib zur Speisung hingebe, damit ich rühmen

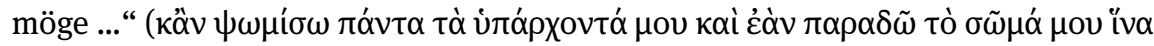

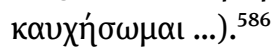

Zuletzt ist auch noch auf 1 . Kor 8,13 zu verweisen, wo der Konjunktiv mit oú $\mu \eta \dot{z}$ zur stärkstmöglichen Verneinung zukünftigen Geschehens gebraucht wird:

584 Auch wenn nicht ganz klar ist, welches Ziel es genau ist, das er damit verfolgt.

585 So Schmeller, Brief II, 236 mit der ganz richtigen Wahrnehmung des Kommunizierten.

586 Die Konjunktive im Konditionalsatz sind unproblematisch, derjenige im ï $\alpha$-Satz ist aber

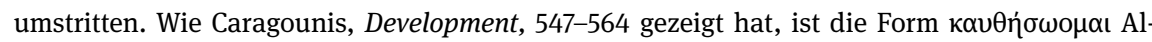

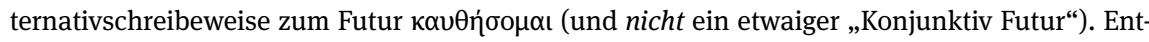
sprechend sind die Textzeugnisse gemeinsam zu werten, womit die Lesart ,... damit mein Leib verbrannt würde ...“ stark an Plausibilität gewinnt. Vermutlich liegt hier also Indikativ Futur im ǐvo-Satz vor. Vgl. hierzu auch Kapitel 12, Abschnitt 3.2 zu Röm 10,14. 
Gemäß dem Fall, dass eine Speise den Bruder zum Fall bringen könnte (indefi-

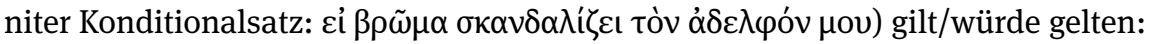

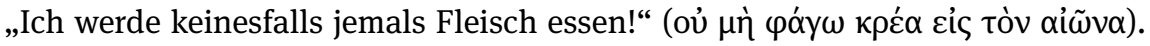
Damit würde das Ziel verfolgt, dass ,ich meinen Bruder nicht zu Fall bringen

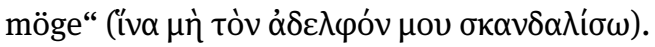

\subsubsection{Bei auf die Vergangenheit zurückblickender Mittel-Proposition}

Nicht zu vernachlässigen sind im Kontext der zukunftsungewissen Vorausdeutungen auf die Zukunft des Erzählers aber auch die in der Vergangenheit lokalisierten Handlungen, die als Mittel eines Zwecks spezifiziert werden, wobei das Eintreten oder Nichteintreten im Text nicht thematisiert wird, da es aus Sicht des

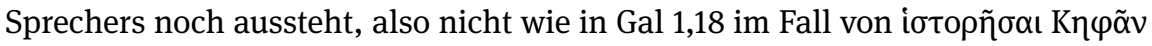
eine zukunftsungewisse Vorausdeutung auf die Vergangenheit des Erzählers vorliegt. ${ }^{587}$

Dem großen Potenzial für die narratologische Analyse steht freilich die größere Schwierigkeit in der Bestimmung des Zukunftsbezugs gegenüber. Am deutlichsten ist diese Konstellation zu erkennen, wenn sich das Ziel auf endzeitliche Ereignisse bezieht. Dies ist beispielsweise in 2. Kor 11,2 der Fall. Hier verwendet Paulus das Bild der Verlobung, um seine missionarische Tätigkeit in Korinth aufzugreifen: Er habe die Korinther mit „einem einzigen Mann verlobt“

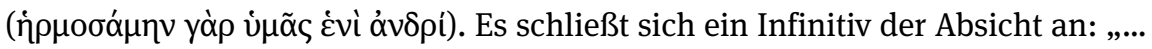

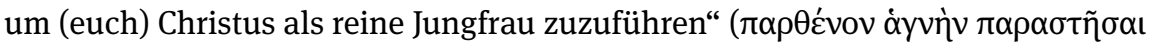
$\tau \tilde{\omega}$ X $\rho ı \tau \tilde{\omega})$. Damit ist auf die eigentliche Vermählung bei der Parusie des Messias Bezug genommen. ${ }^{588}$ Diese finale Konnexion wiederum stützt kausal (yó $\rho$ ) die vorangehende Aussage, wonach Paulus um die Korinther mit Gottes Eifer eifre

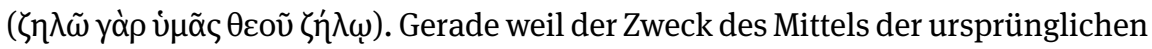
Missionierung in der Zukunft liegt, ist das Erreichen des Zieles noch nicht gesichert und - so macht 11,3 durch einen Befürchtungssatz deutlich ${ }^{589}$ - momentan in Gefahr. Der Eifer tritt somit als Mittel zur Gewährleistung des Erreichens des ursprünglich anvisierten Ziels hinzu.

Nicht immer ist der Zukunftsbezug so klar erkennbar. Die Offenheit kann dabei durchaus auch eine kommunikative Funktion erfüllen. So schildert Paulus

587 Siehe oben, Kapitel 8, Abschnitt 3.6 zum elliptischen Erzählen. Siehe auch Kapitel 11, Abschnitt 6 zu Vorausdeutungen auf die Vergangenheit des Erzählers im Allgemeinen. Vgl. auch dort in Abschnitt 6.3 die Diskussion zur besonderen Konstellation in Gal 2,5, abhängig von der angenommenen Datierung/Adressatenschaft.

588 Vgl. zu den Bildern und den alttestamentlichen Hintergründen Schmeller, Brief II, 199-201. 589 Zum genauen Inhalt des Befürchteten siehe oben Abschnitt 3.5.1 zur Stelle bei den Befürchtungssätzen. 
in Phlm 13 zunächst ein in der Vergangenheit liegendes Begehren: ${ }^{590}$ Er wollte One-

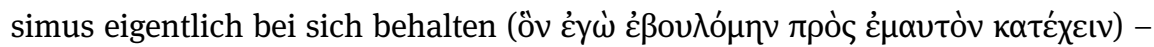
und zwar zu einem bestimmten Zweck: damit dieser ihm anstelle von Philemon

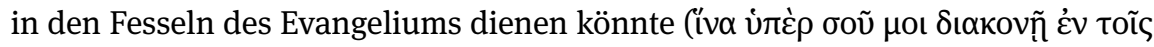

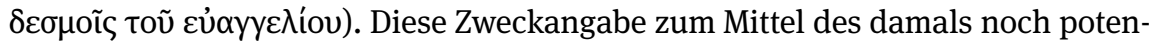
ziellen (nur gewollten) „Dabehaltens“ muss aus der Perspektive des Briefschreibers in der noch ausstehenden Zukunft liegen - denn bis zu diesem Punkt der Sendung des Schreibens hatte Paulus Onesimus ja für sich. V. 14 schildert dann den tatsächlich getroffenen Entschluss, der dieser persönlichen Präferenz entgegenlief - und somit die genannte Zukunft mit Verweis auf ein vergangenes Ereig-

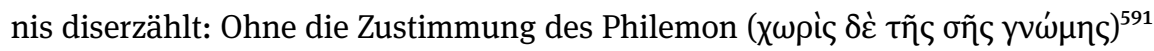

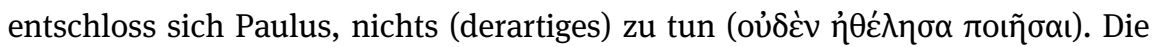
Finalangabe $\mathrm{zu}$ diesem mentalen Geschehen in der Vergangenheit lautet: „... damit deine Wohltat nicht wie aus Zwang, sondern freiwillig wäre“ (îv $\mu$ ทे $\dot{\omega} \varsigma$

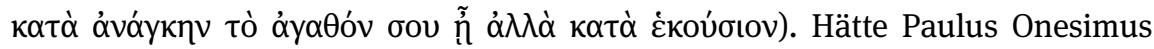
einfach bei sich behalten, hätte er damit Philemon indirekt zu etwas gezwungen, da dieser keine Wahl gehabt hätte. Genau diese Folge galt es aus der Sicht des Paulus jedoch zu vermeiden. Da Paulus Onesimus nun zurückschickt, wird es entsprechend auch nicht zu dieser Situation kommen.

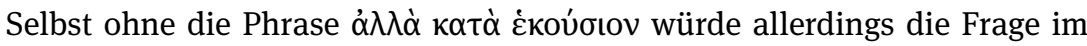
Raum bestehen bleiben: Und was ist nun mit „Deiner Wohltat“ ( Das hypothetische Ereignis, dass Paulus von Philemon durch Onesimus profitieren könnte, ist ja nicht als Ganzes diserzählt. Auch wenn Paulus in V. 15-16 die Möglichkeit einräumt, dass der „Sinn“ der Ereignisse darin bestehen könnte, dass Philemon Onesimus nun für immer als werten Bruder zurückerhalte, und auch wenn die schlussendlich doch noch erfolgende expliziten (Nicht-)Anordnung ${ }^{592}$ in V. 17 genau darauf Bezug nimmt, kann daher kaum ein Zweifel daran bestehen, dass in V. 21 das „Wissen“ des Paulus, „dass Du mehr tun wirst, als ich

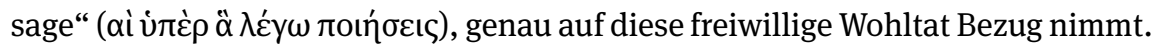

\subsubsection{Aorist des Briefstils in der Mittel-Proposition}

In den bisher besprochenen Fällen zeigt das Augmenttempus in der MittelProposition an, dass das Geschehen in der Vergangenheit des Sprechers zu

$590 \mathrm{Zu}$ den Tempora vgl. Burton 33. Vgl. zu den Deutungsoptionen für diese Passage auch bereits oben, Abschnitt 4.3.2 (Sprechakt „Wunsch“) und 4.4.2.2 (Aussage über vergangenen mentalen Zustandes „Wunsch“).

591 Für yvú $\mu \eta$ im Sinne von ,Zustimmung، siehe LN 31.14.

592 Siehe hierzu oben, Abschnitt 4.4.2. 
verorten ist. Der Sonderfall des Aorists des Briefstils, welcher die „Verwirklichung des Verbinhalts ... aus der [Sicht] des Empfängers (als vergangen) darstellt,“593 ist ebenfalls in diesem Kontext zu beachten: Das aus Sicht des Schreibers sich im Verlauf befindliche oder unmittelbar bevorstehende Geschehen wird durch den indikativischen Aorist in der Vergangenheit der Briefempfänger lokalisiert, während die in manchen Fällen angefügte Zweck-Proposition wiederum auf die Gegenwart des Lesens oder ein sich anschließendes Zeitintervall Bezug nimmt.

Natürlich kann das Ziel im Einzelfall auch hier bereits vor dem Akt der Lektüre verwirklicht sein - so vermutlich in Phil 2,28: „Ich habe ihn nun besonders eilig gesandt, damit ihr - wenn ihr ihn seht - wieder froh und ich sorgenfreier würdet“

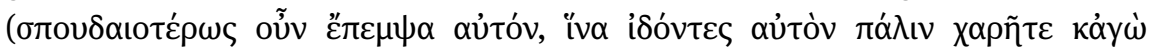

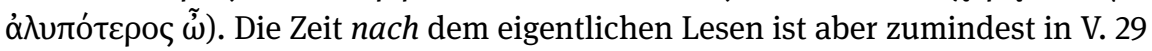
im Blick, wo Paulus unter anderem - im Medium des Briefes - zur entsprechen-

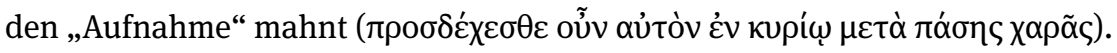

Zumindest teilweise an ein noch ausstehendes Geschehen dürfte in Kol 4,8 gedacht sein: „Den habe ich zu euch gesandt zu dem Zweck, damit ihr das mich Betreffende erfahren möget und damit er [Tychikus] eure Herzen tröste“ (öv

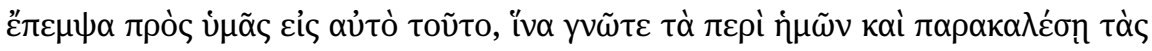

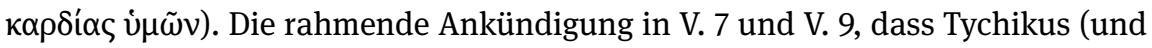
Onesimus) die Paulus betreffenden Umstände „kundtun wird/werden“ (V. 7: Tò

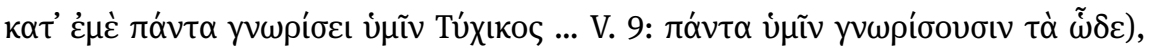
fungiert nicht nur als Beglaubigung des von diesen bis dahin Übermittelten, sondern indirekt auch zur Aufforderung, sich nach der Lektüre mit verbliebenen Fragen vertrauensvoll an die Gesandten zu wenden. ${ }^{594}$

In Eph 6,22 wird die Formulierung aus Kol 4,8 wortwörtlich wiederholt (ôv

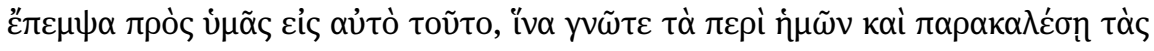

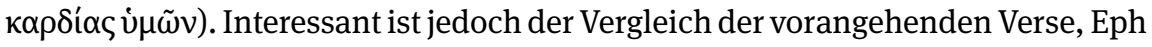
6,21 mit Kol 4,7: Tychikus wird mit einer nahezu identisch ausgestalteten Apposi-

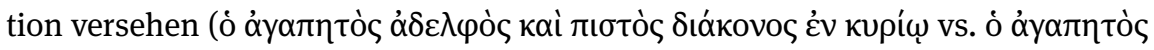

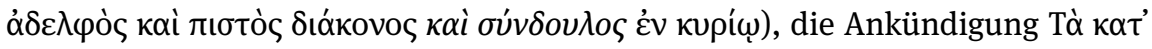

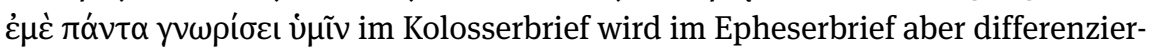
ter aufgegriffen. Angekündigt wird, dass er den Lesern „alles“ kundtun werde

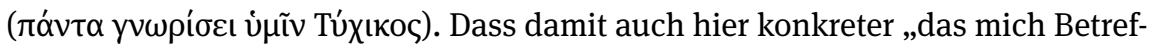
fende“ gemeinst ist, wird durch einen vorangestellten Finalsatz ausgedrückt, in welchen dieser Präpositionalausdruck integriert ist: „Damit aber auch ihr das

593 GGNT 199j. Siehe dazu ausführlich oben, Kapitel 6, Abschnitt 3.4.2.1 und Kapitel 11, Abschnitt 1.

$594 \mathrm{Zu}$ den Komplexitäten dieser Interaktion zwischen Gemeinde und Briefüberbringer/-in siehe nun als aufschlussreiches narratives Experiment Gooder, Phoebe. 


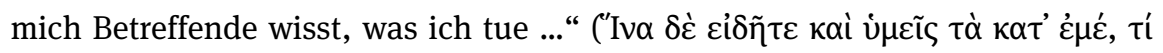
$\pi \rho \alpha ́ \sigma \sigma \omega)$. Viele Kommentatoren lassen die genaue Art der Umarbeitung jedoch

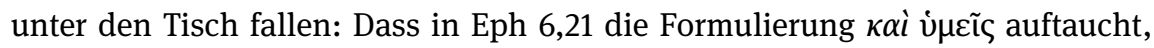
dürfe in ihren Augen nicht so verstanden werden, dass hiermit im Sinne einer Echtheitshypothese „auf einen anderen Paulusbrief aus der gleichen Situation“ verwiesen werden solle. ${ }^{595}$

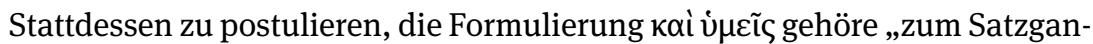
zen und stell[e] die Adressaten nur dem Apostel gegenüber“"596 ignoriert jedoch vollkommen, dass dieser Aspekt schon vom Weltwissen her (natürlich weiß Paulus über seine eigene Situation Bescheid!) gegeben ist. Darin unterscheidet sich diese Stelle von den typischerweise hier angegebenen „Parallelen“597 wie Röm 8,11, wo es hervorragenden Sinn macht, dass Paulus von „auch euren“ sterblichen Leibern und deren Auferweckung spricht, nachdem er zuvor die Auf-

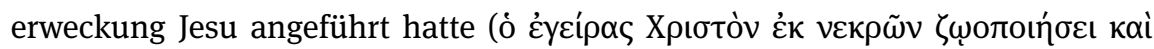

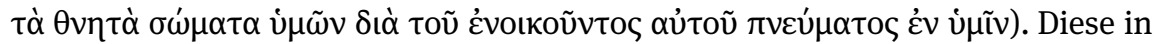
vielen Kommentaren ${ }^{598}$ regelmäßig angebotene Interpretation ist nicht nur syntaktisch unhaltbar, sondern sie verdeckt auch die Tatsache, dass die genannte Differenzierung zwischen Apostel und Empfängern bereits mehr als deutlich

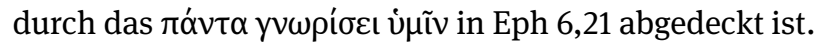

Es muss also wohl in der Tat davon ausgegangen werden, dass die Empfänger des Briefes hier den Christen in Kolossä zur Seite gestellt werden. Gerade wenn man davon ausgeht, dass der Autor des Epheserbriefes Kol 4,7 direkt vorliegen hatte, kann erwogen werden, ob der Autor hier schlicht unbewusst aufgrund des ihm vorliegenden weiteren Briefes so formuliert. Allerdings ist die Ergänzung um kaí nicht in Isolation zu betrachten. Das indikativische Verb mit indirektem

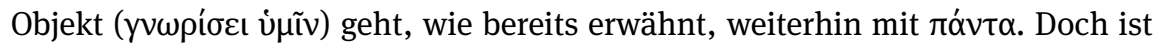

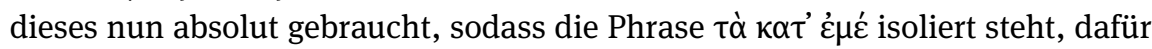

595 Schnackenburg, Epheser, 295.

596 Schweizer, Brief, 295.

597 Wenn schon, dann müsste man wohl mit Stellen wie Homer, Ilias 16.19 arbeiten: „Sprich,

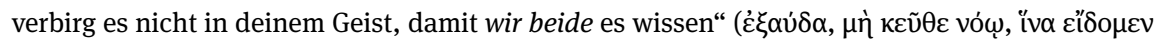
å $\mu \varphi \omega)$.

598 Bei Schnackenburg, Epheser, 293 bringt zudem die Übersetzung eine völlig andere Schwerpunktsetzung zum Ausdruck: „Damit ihr aber auch, was mich betrifft, wißt, wie es mir geht ...“ Liegt hier der Satzakzent auf „wißt“? Sollen die Empfänger es also „auch wissen“ und nicht nur vermuten? Die NGÜ demonstriert noch eine weitere Möglichkeit, die im Griechischen ganz eindeutige Schwerpunktsetzung, die in der Randnotiz auch richtig als Alternative angegeben wird, zu verkennen: „Nun werdet ihr aber auch etwas über mich erfahren wollen ...“ Bei Schnackenburg scheint ein solcher Satzakzent nicht möglich: Man kann schließlich nicht * ,was nicht mich betrifft, wissen, wie es mir geht.“ 


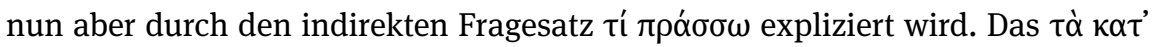

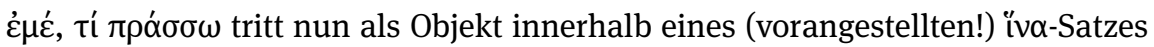
mit oĩ $\delta \alpha$ auf: „Damit auch ihr das mich Betreffende erfahrt, wie es mir geht ${ }^{599}$...“

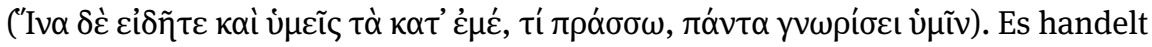
sich also bei dieser sorgfältigen Umarbeitung keinesfalls nur um eine Hinzufü-

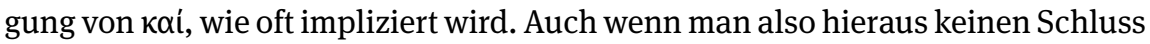
über die tatsächliche Abfassungssituation ableiten möchte, wird man wohl kaum darum herumkommen, einzuräumen, dass zumindest innerhalb der erzählten Welt von Eph 6,21 bewusst eine weitere Botenfunktion des Tychikus vorausgesetzt ist (vgl. auch Kol 4,16).

In 2. Kor 9,3 schließlich ist das ins Auge gefasste Geschehen deutlich als der Lektüre des Briefes nachfolgend zu verstehen. Das Mittel stellt hier die Sendung der Brüder dar, welche mit dem Zweck erfolgt, die Sammlung zu einem guten Ende zu bringen. Der zeitliche Horizont ist in 9,4 durch das Kommen des Paulus

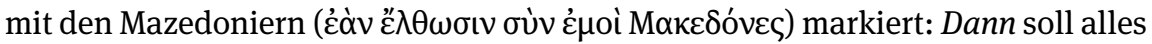

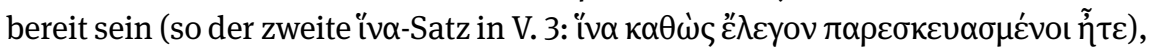
damit das Rühmen der Korinther sich nicht nachträglich als haltlos herausstellt

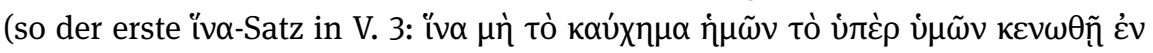
$\left.\tau \tilde{\omega} \mu \varepsilon^{\prime} \rho \varepsilon \iota ~ \tau o u ́ \tau \omega\right)$ ). Vers 4 formuliert ebenfalls negativ (diesmal mit $\mu \eta \dot{\eta} \pi \omega \varsigma$ ), greift nun aber direkt das Gegenstück des zweiten, positiv formulierten, Ǐv $\alpha$-Satzes auf: „damit wir - um nicht zu sagen: ihr - nicht zuschanden werden in dieser

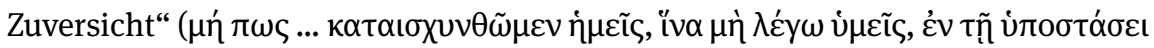
$\tau \alpha u ́ \tau \eta ̣)$. Der Fall wäre dies, wenn die Bedingung einträte, dass die mazedonischen

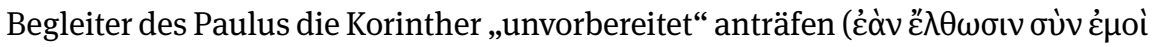

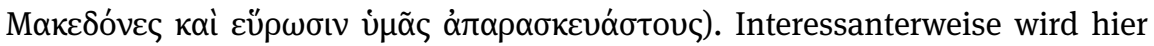
also gar nicht der unmittelbare Zweck thematisiert, $\mathrm{zu}$ welchem die Delegation gesandt wird. (Auch 9,5 bleibt verhältnismäßig vage.) Vielmehr wird das sich an die Überbringung des Briefes anschließende, hier aber nicht näher ausgeführte Handeln selbst wiederum als Mittel zu einem Folgezweck qualifiziert.

Ein wenig anders gelagert ist das Verhältnis von Mittel, unmittelbarem Zweck und Folgezweck in 1. Kor 4,16-17. Die Sendung des Timotheus wird als Resultat

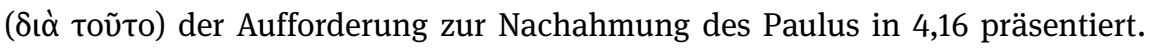
Gemeint ist wohl: Der der Aufforderung zugrundeliegende Wunsch des Paulus

599 Es gehört zu den absonderlichen Blüten der (von Caragounis, Development und New Testament trotz allem überflüssigen Pathos zurecht monierten) erstaunlichen Gleichgültigkeit der heutigen Exegese gegenüber der diachronen Entwicklung des Griechischen, dass hier von Schnackenburg, Epheser, 295 für „ähnliche[n] Sprachgebrauch auch in modernen Sprachen“

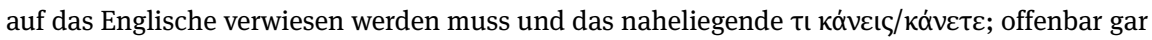
nicht bekannt zu sein scheint. 
bringt ihn auch dazu, Timotheus zu senden. Der Zweck, zu dem diese Sendung das Mittel darstellt, wird durch den Relativsatz mit finalem Nebensinn ${ }^{600}$ angegeben: Er besteht im Akt des Erinnerns an „meine Wege in Christus“ (ò ک ú $\mu \tilde{\alpha} \varsigma$

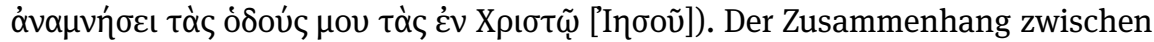
kausaler Basis des Sendens und dessen Zweck besteht nun darin, dass durch diese Erinnerung aus 4,17 die Nachahmung aus 4,16 erleichtert werden soll. Der Folgezweck (die Nachahmung selbst) wird hier dann nicht separat mit weiterem iv $\alpha$-Satz angeknüpft, da er innerhalb der vorangehenden kausalen Konnexion bereits vorausgreifend thematisiert worden ist.

\subsubsection{Beispiel Gal 1,4}

Der Finalsatz in Gal 1,4 illustriert das Problem der zeitlichen Referenz von ZWECK-Propositionen eindrücklich. Im Folgenden soll die These vertreten werden, dass hier das Erreichen des Zwecks bewusst offen gelassen wird. Die Diskussion dieser Textstelle soll hier etwas ausführlicher durchgeführt werden, da es sich hierbei um eine für die Paulusforschung recht zentrale Passage handelt und sich an ihr zugleich viele grundlegende Erwägungen im Umgang mit Finalangaben als mögliche Erzählfragmente impliziter, in die Zukunft verweisender Erzählungen verdeutlichen lassen.

Der Vers Gal 1,4 ist insbesondere für die „apokalyptische“ Paulusauslegung von großer Bedeutung. In seinem wegweisenden Aufsatz zur Apokalyptik im Galaterbrief ging Martyn von der Rede einer „neuen Schöpfung“ in Gal 6,13-14 aus: Dass aus dieser die Nichtigkeit von Beschneidung und Unbeschnittenheit folge, liege daran, dass nicht etwa eine alte heilsgeschichtliche Periode zu Ende gegangen, sondern die alte Welt mit den sie (im antiken Weltbild) konstituierenden „Gegensatzpaaren“ aufgelöst sei. ${ }^{601}$ Gegen Ende seines Aufsatzes kommt Martyn dann aber auch auf Gal 1,4 zu sprechen - als Beleg für eine primär in der Kreuzigung angesiedelte „Apokalypse.“602 Hierin liegt freilich der Hauptunterschied in der Verwendung der apokalyptischen Terminologie zu Martyns Lehrer Käsemann, in dessen Tradition er sich mit dieser Arbeit sieht. ${ }^{603}$

Martyns Schüler de Boer wiederum betont, dass die eigentliche Front bei Paulus zwischen $z$ wei verschiedenen Formen apokalyptischen Denkens bestehe: ${ }^{604}$

\footnotetext{
$\mathbf{6 0 0}$ Vgl. oben, Abschnitt 5.3.1.

601 Martyn, „Antinomies,“ 412-415.

602 Martyn, „Antinomies,“ 420.

603 Siehe insbesondere die Endnoten 2-6 bei Martyn, „Antinomies,“ 421 für den Rückbezug auf Käsemann.

604 de Boer, „Apocalyptic Eschatology.“
} 
Sowohl das Paradigma der Anthropologie von Bultmann als auch das Paradigma der Kosmologie von Käsemann sei „apokalyptisch“ und sowohl im Judentum als auch Seite an Seite bei Paulus anzutreffen. Jedoch zeige eine Analyse des Römerbriefs, dass Paulus selbst die kosmologische Eschatologie des zweiten Ansatzes bevorzuge und die forensisch orientierten Motive (die der anthropologischen Denkweise zugeordnet werden) nur aufgrund seiner Gesprächspartner in Rom aufgreife. ${ }^{605}$ Ebenso seien auch die „Teacher“ im Galaterbrief letztlich ,apokalyptisch“ - jedoch eben nach dem forensischen Muster, das Paulus im Brief letztlich durch die kosmologische Variante zu überwinden versuche. ${ }^{606}$

Entsprechend wird von den beiden Autoren die attributive Näherbestimmung des Herrn Jesus Christus aus V. 3, die in V. 4 erfolgt, interpretiert: Dessen Charakte-

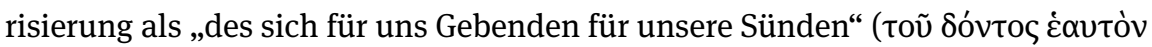

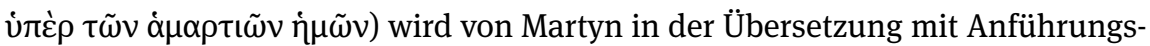
zeichen versehen, um es als judenchristliches Traditionsgut $\mathrm{zu}$ markieren. ${ }^{607}$ Auch wenn die genaue Quelle letztlich offen bleibt, sei doch ein Punkt „certain“: „The formula is to a significant degree foreign to Paul's own theology. “608 Der anschließende ő $\pi \omega \varsigma$-Satz „korrigiere“ dieses Verständnis: Paulus „is now himself the author of the final clause. "609 Zum Ausdruck komme hier die grundsätzliche paulinische Überzeugung ,that for him the motif of cosmic warfare is focused first of all on the cross ... There, in the thoroughly real event of Christ's crucifixion, God's war of liberation was commenced and decisvely settled, making the cross the foundation of Paul's apocalyptic theology. “610

Bei de Boer wird ähnlich betont, dass auf die knappe Partizipialkonstruktion eben gerade nicht die Rede von „Vergebung“ folge: „Paul immediately interprets Christ's giving himself ,for our sins' to effect not forgiveness but deliverance from an evil realm. “611 Dies steht natürlich in Übereinstimmung mit de Boers Identifikation von zwei verschiedenen apokalyptischen Verständnisweisen: „Paul thus shifts the import of the phrase ,for our sins ${ }^{6}$ from a forensic (judicial) frame of reference (the divine lawcourt) to a cosmological one (a cosmic conflict between God and malevolent powers for sovereignty over the human world).“612

605 de Boer, „Apocalyptic Eschatology,“ 182-184.

606 de Boer, „Apocalyptic Eschatology,“ 184-185.

607 Martyn, Galatians, 89.

608 Martyn, Galatians, 90.

609 Martyn, Galatians, 90.

610 Martyn, Galatians, 101.

611 de Boer, Galatians, 30.

612 de Boer, Galatians, 30. 
Während es bei Martyn also zu einer expliziten Verwerfung der Partizipialkonstruktion kommt, handelt es sich nach de Boers Verständnis um eine Reinterpretation. Gemeinsam ist beiden Verständnisweisen, dass der Hauptakzent

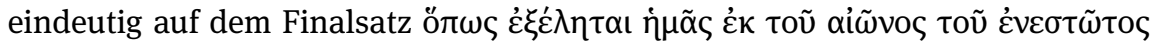

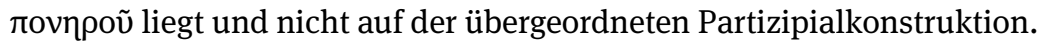

Wie ist dieses Geschehen nun zeitlich zu verorten? Die meisten Exegeten beobachten, dass in der Rede vom „gegenwärtigen bösen Zeitalter“613 ein Motiv jüdischer Apokalyptik aufgenommen werde, welches zwischen dem gegenwärtigen und kommenden Äon unterscheide. ${ }^{614}$ Vor dem Hintergrund der Annahme einer „typical NT perspective of inaugurated eschatology“615 wird dann hier gerade in der zeitlichen - letztlich eschatologischen - Motivik ein Verweis auf die Vergangenheit gesehen: „Paul claims that, though this present evil age continues in force, believers are rescued from this present age of evil, sin, and death and find their true identity in the new age that has broken into history through Christ's epochal death and resurrection." 616

So eindeutig ist der Text allerdings nicht. Dies beginnt bereits damit, dass die vorausgehende Rede von der Hingabe Christi in einer Partizipialkonstruktion erfolgt, die entsprechend natürlich keine Zeit, sondern lediglich Aspekt zum Ausdruck bringt. Nicht nachvollziehbar ist daher, dass Martyn behauptet: „In the main clause of the sentence Paul has followed the atonement formula in speaking of an event lying in the past: Christ gave up his life for our sins. “617 Schon diese Voraussetzung ist nicht haltbar. Der „Hauptsatz“ ist freilich die elliptische Konstruktion in V. 3 (mit zu ergänzendem عi̋n) und der Vergangenheitsbezug wird ausschließlich in Martyns indikativischer Übersetzung codiert.

Korrekt wäre also vielmehr die weitaus vorsichtigere Aussage, dass der das Geschehen als Ganzes perspektivierende Aorist eine Verbindung des zum Ausdruck gebrachten Verbinhalts mit einer konkreten Referenz in der Vergangenheit - der Kreuzigung - problemlos erlaubt und diese Verbindung aufgrund frühchristlichem Sprachgebrauch als sehr plausibel zu gelten hat. Grammatisch spricht freilich nichts dagegen, dass Jesus als „Geber“ charakterisiert wird, der sich als solcher in vielen Einzelakten - evtl. auch noch immer anhaltenden - als solcher erweist.

613 Zum Text siehe Carlson, Text, 183.

614 Vgl. aber Frey, „Demythologizing Apocalyptic?,“ 514, der darauf hinweist, dass Paulusforscher häufig übersehen, dass mit dieser temporalen Orientierung lediglich ein Teil der Apokalypsen treffend charakterisiert sei.

615 So Moo, Galatians, 73.

616 Moo, Galatians, 73.

617 Martyn, Galatians, 90. 
Ebenso wenig grammatisch folgerichtig ist freilich auch die Konsequenz, die Martyn zieht: ${ }^{618}$

\begin{abstract}
He uses the verb ,to snatch out of the grasp of ' ... with the meaning ,to deliver, to rescue, and he employs a clause form (hopôs with the aorist subjunctive) that takes its temporal import from the verb of the main clause ... The result is a sentence in which Christ's death is interpreted as the past event that in itself accomplished not only the forgiveness of our sins but also and climatically our deliverance from a foreign power (the present evil age).
\end{abstract}

Eine automatische „Übernahme“ des Zeitbezuges gibt es nicht - weil das gemeinte Verb keinen Zeitbezug codiert, aber auch grundsätzlich, weil die besagte Relation sehr vielgestaltig sein kann, wie in der vorangehenden Diskussion ja auch skizziert wurde.

Auffällig ist daher also vielmehr, dass der Autor an dieser Stelle gerade nicht indikativisch formuliert, anders also als der Autor im Fall des teilweise Syno-

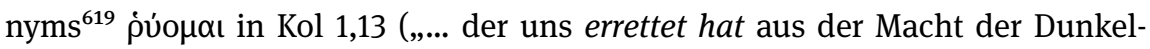

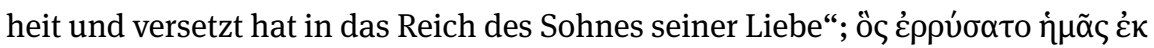

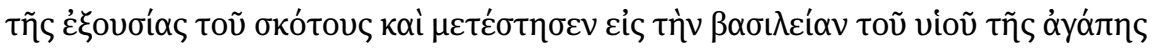

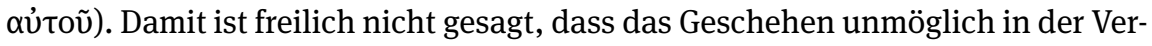
gangenheit liegen kann. Der Vergangenheitsbezug kann aber auf jeden Fall nicht aus der Syntax selbst abgeleitet werden.

Sucht man nach Hinweisen für die korrekte Interpretation des in Gal 1,4 Gemeinten, ist weiterhin die Beobachtung relevant, dass Paulus an anderen Stellen durchaus Verben aus dieser semantischen Domäne ,Cause to be Safe, Free from Danger (LN 21.17-24) in ǐva-Sätzen verwenden kann, um auf Zukünftiges $\mathrm{zu}$ verweisen. Zugleich ist dann freilich auch genau darauf zu achten, wie dort der Zukunftsbezug kommuniziert wird. Die genauere Betrachtung zeigt nämlich, dass dieser in der Regel durch recht deutliche Kontextfaktoren gesichert ist - und diese so im Galaterbrief keine exakte Entsprechung haben. ${ }^{620}$

618 Martyn, Galatians, 90. Kursive Hervorhebungen (außerhalb der Transliteration) nicht im Original.

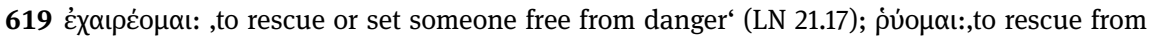
danger, with the implication that the danger in question is severe and acute، (LN 21.23).

620 In Röm 15,31 ergibt sich die Zukunftsperspektive daraus, dass das Mittel des Gebets in V. 30 selbst erst noch Teil einer Aufforderung ist, dessen Verwirklichung also noch aussteht - und damit natürlich auch das Erreichen des Ziels. Zugleich ist dieses Ziel der Rettung konkret als eine

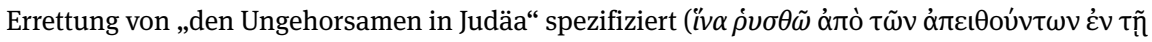

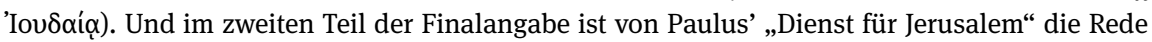

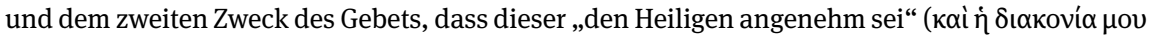

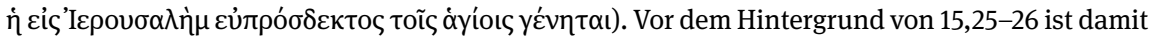


bei allen offenen Details eindeutig auf die noch ausstehende Jerusalem-Reise Bezug genommen. Vgl. zur Passage oben, Kapitel 11, Abschnitt 8.

Etwas weniger deutlich ist die Situation in 1. Kor 10,33. Hier folgt auf den konativen Indi-

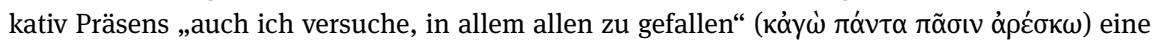
Konstruktion mit modalem Partizip: „indem ich nicht meinen Vorteil suche, sondern den der

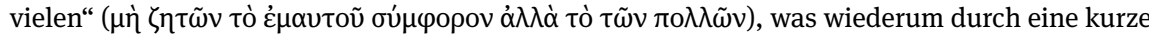

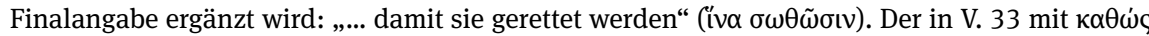
eingeleitete Vergleichssatz liefert den Hintergrund für die Aufforderung zum unanstößigen Verhalten gegenüber Juden, Griechen und der Gemeinde in V. 32. Es ist hier also sicherlich an Paulus' generelle Praxis gedacht und zukünftig ist das im Finalsatz geäußerte Ziel nur insofern, als er auch noch für die Zukunft davon ausgeht, Individuen zu treffen, die zu den ,vielen“ gehören.

Dies gilt wohl auch im Hinblick auf das „Reden zu den Nationen,“ an welchem Pau-

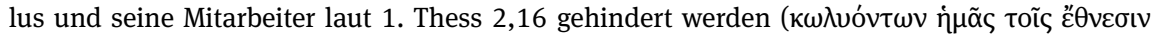
$\lambda \alpha \lambda \tilde{\eta} \sigma \alpha \mathrm{l})$, auch wenn hier der Zeitbezug aufgrund der komplizierteren Syntax nicht so eindeutig ist. Auf jeden Fall erfolgt das „Reden“ auch hier mit dem Zweck „damit sie gerettet werden“ (ív $\sigma \omega \theta \tilde{\omega} \sigma \iota v)$. Das übergeordnete adverbiale Partizip von $\kappa \omega \lambda v \dot{\omega} \omega$ (,hindern“) bringt durch den Durativ-Stamm vermutlich einen konativen Aspekt zum Ausdruck (NSS 1097) und hat natürlich keine Zeitbedeutung. Der sich anschließende Satz „Aber der Zorn ist schließlich [?] über

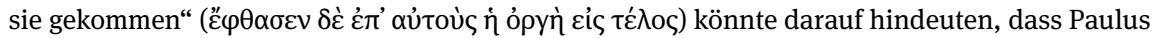
hier vor allem die bisherige Verfolgung - gerade auch in Thessaloniki selbst (Apg 17,5) - vor Augen hat. Allerdings ist der Aorist wohl als „proleptisch“ zu verstehen, also mit Zukunftsbezug zum Ausdruck der Sicherheit des nahenden Gerichts. Dies erlaubt auch eine etwas bessere Einordnung der (vermutlich konsekutiven) zwischengeschalteten Infinitiv-Konstruktion: Aus der Hinderung der Mission folgt, „dass sie immer das Maß ihrer Sünden vollmachen“ (દí tò

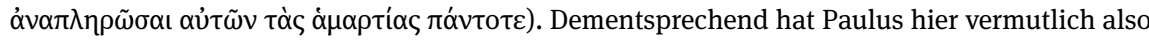
auch noch zukünftige Bedrängnis im Blick (deren sicheres Ende er jedoch bereits antizipiert) und damit auch noch anhaltendes „Reden“ mit dem weiterhin verfolgten Zweck der „Rettung.“

Auch in 1. Kor 9,22 ist das Ziel als letztlich in der Zukunft liegend zu verstehen gemeint, da der Finalsatz hier auf die Beschreibung eines gegenwärtigen Zustands folgt. Die Konstruktion ist im Kontext äußerst auffällig: In V. 19 verwendet Paulus den Indikativ Aorist, um auf sein „Sich-selbst-zum-Sklaven-Machen“ Bezug zu nehmen ( $\varepsilon \dot{\delta} \delta u^{\prime} \lambda \omega \sigma \alpha$ ). Es folgt in 9,20 und 9,22a ebenfalls im Aorist ćycvó $\mu \eta v$ (,ich wurde“), wobei in den Versen 19-22a insgesamt fünf Mal ein Finalsatz „damit ich ... gewinnen möge“ (iv $\alpha \kappa \varepsilon \rho \delta \eta \dot{\sigma} \sigma \omega)$ angefügt wird. Man kann die Einordnung der Aorist-Formen als „gnomisch“ erwägen. (So Robertson, Grammar, 843; vgl. zur Kategorie oben, Kapitel 6, Abschnitt 3.4.2.4). Auf jeden Fall ist der Wechsel zum Präsens im folgenden Vers 23 auffällig: „Ich tue aber alles wegen des Evangeliums, um Anteil an ihm zu bekommen“

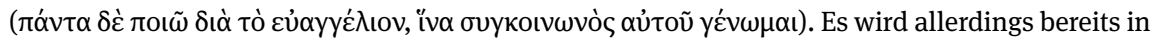
der Zusammenfassung in V. 22b (zur Beschreibung des Zwecks dient nun das Verb $\sigma \dot{\omega} \zeta \omega$ ) durch den Wechsel in den Ind. Perf. von yivo $\mu \alpha$ ein Fokus auf die Gegenwart vorgenommen: „Ich bin

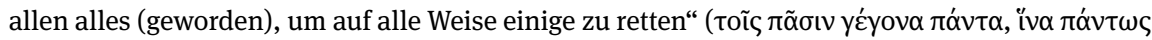

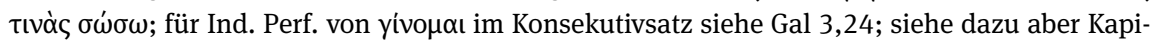
tel 6, Abschnitt 3.3.2.3). Der gegenwärtige Zustand ist hier das Mittel zum Erreichen des noch ausstehenden Ziels. Die Übersetzungen verkennen dieses finale Verhältnis in der Regel aufgrund der Schwierigkeit der Wiedergabe des griechischen Perfekts im Deutschen: Sie erwecken den Anschein, der Finalsatz liefere die Basis für die Folge des nun eingetretenen Zustands. Dadurch, 
Eine Passage, die sich als Parallele sehr nahelegt, ist allerdings 1. Thess 5,10. Sie ist syntaktisch ganz analog konstruiert und zudem weist die PartizipialKonstruktion dieselbe Referenz auf wie in Gal 1,4. Hier wird „unser Herr Jesus Christus“ aus dem Vorvers zunächst beschrieben als „,der für uns Gestorbene“ ( $\tau$ ov

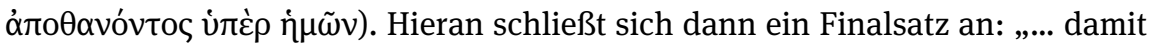

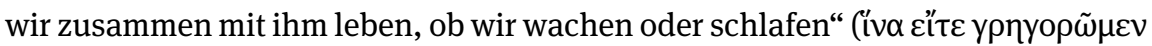

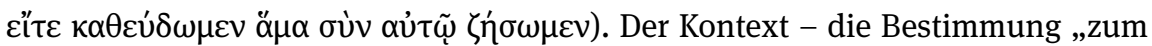

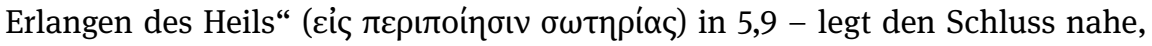
dass mit dieser Metapher gemeint ist: Während Paulus in 4,13-17 von „uns“ Lebenden und den Entschlafenen spricht, verwendet er nun die 1. Pl., um die beiden Möglichkeiten aufzugreifen, bei der Ankunft des Herrn noch lebend oder bereits tot zu sein. Das „Mit-ihm-Leben“ wäre folglich referenzidentisch mit dem

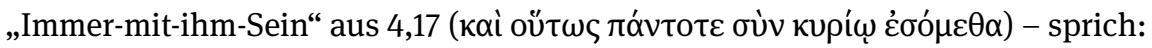
eschatologisch.

Beachtet man (a) die Parallelität von Gal 1,4 mit 1. Thess 5,10 und zieht (b) eine Verbindung zwischen dem Finalsatz in 1. Thess 5,10 mit dem Endzustand in 4,17, so liegt natürlich die Vermutung nahe, dass der Finalsatz in Gal 1,4 Zukunftsbezug aufweist, vielleicht sogar durch die Rede vom „Herausreißen“ an das

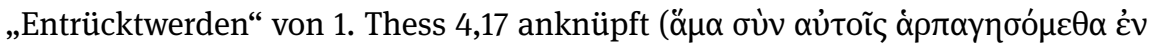

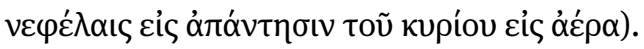

Eine solche Auslegung ist besonders dann attraktiv, wenn man für Gal 1,4 keine temporale, sondern eine räumliche Dichotomie voraussetzt. So hat Grind-

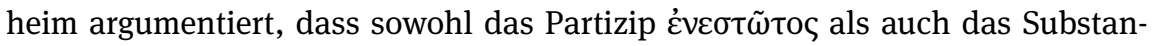
tiv aíuv nicht notwendigerweise ein zeitliches Verständnis erfordere. ${ }^{621}$ In der Tat kann aíwv auch ,the world as a spatial concept" bedeuten. ${ }^{622}$ Leider taucht das Substantiv in antiken Quellen nahezu nie in aussagekräftiger Verbindung

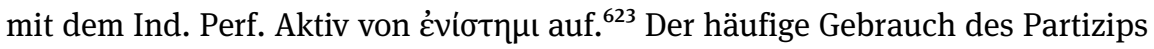
mit anderer eindeutig temporaler Terminologie, ${ }^{624}$ lässt es allerdings zumindest sehr zweifelhaft erscheinen (auch wenn eine Analyse tatsächlich etablierter

dass Paulus in der Vergangenheit besagte Absicht verfolgte, ist er jetzt jemand, der dadurch gekennzeichnet ist, dass er in der Zwischenzeit allen alles geworden ist.

621 Grindheim, „Salvation Territory,“ 93-95.

622 BDAG 233,3.

623 Eine der seltenen Ausnahmen ist ein Fragment von Numenios, das bei Eusebios, Praep. ev. 11.10.2 überliefert ist: „Wenn nun jemand wünscht, dieses Gegenwärtige ,Ewigkeit‘ zu nennen,

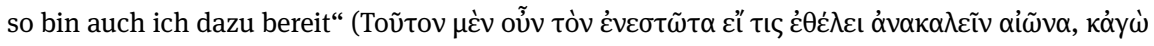
бu$\mu \beta о и ́ \lambda о \mu \alpha \mathrm{l})$.

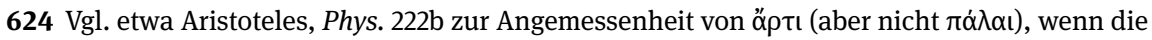
gemeinte Zeit „nah am Jetzt“ liege ( 
Kollokationen noch angezeigt wäre), dass aíwv in Gal 1,4 ohne zeitliche Bedeutung verwendet werden könnte.

Die Parallelität zu 1. Thess 5,10 (+4,17) ist aber auf jeden Fall sehr auffällig. Ein eindeutiger Schluss kann auf ihrer Grundlage jedoch nicht gezogen werden, denn Paulus ist recht flexibel in der Art und Weise wie er Zweckangaben an die Schilderung des Todes Jesu anfügt, wie kurz an zwei Beispielen verdeutlicht werden soll.

Interessant ist etwa Röm 14,9, wo der Tod Jesu anders als in Gal 1,4 und 1. Thess 5,10 indikativisch aufgegriffen wird: „Dazu ist Christus gestorben und lebendig geworden: damit er herrsche sowohl über Tote als auch Lebende“ (عis

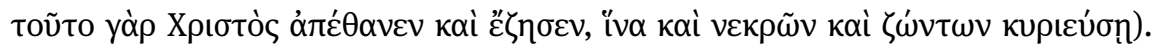
Gemeint ist hier offenbar die Herrschaft über zwei Bereiche, die mit der Auferstehung in der Vergangenheit bereits angetreten wurde. Denn durch diese bereits angetretene doppelte Herrschaft ist gewährleistet, was im Vorvers 14,8 ausgesagt wurde: Sowohl im Fall des Lebens als auch im Fall des Sterbens „sind wir des Herrn, “625 können also nicht in einen Bereich gelangen, der nicht schon von ihm regiert würde.

Auch in 2. Kor 5,15 ist vom Sterben Christi (das Subjekt wird in V. 14 genannt) im Indikativ Aorist die Rede, diesmal wieder (wie in Gal 1,4 und 1. Thess 5,10; nicht so in Röm 14,9) mit der Erklärung, dass das Sterben „für uns“ (alle) geschah

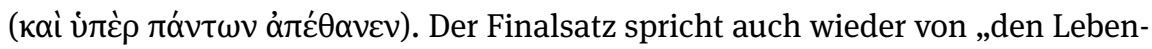
den“ als Subjekt. Doch diesmal stehen diese nicht in einem Kontrast zu einer

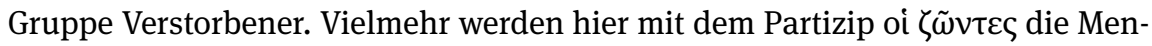
schen als Lebende aufgegriffen, sodass dann im Anschluss der Modus dieses Lebens näher bestimmt werden kann: Der Zweck des Todes Jesu besteht darin, dass sie nun „nicht mehr sich selbst leben, sondern demjenigen, der für sie starb

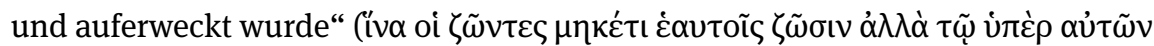

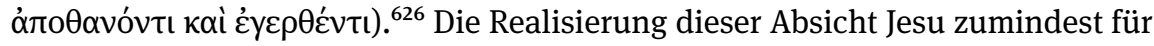
„uns“ (d.h., ein entsprechender Lebenswandel mag bei anderen Personen noch in der Zukunft geschehen und wird auch dann eine entsprechende Zielerfüllung des Todes Jesu sein) zeigt sich in der in V. 16-17 mit zweifachem $̋ \sigma \tau \varepsilon$ eingeleiteten neuen Existenzweise im „Jetzt“ (vũv).

Was also ist mit Gal 1,4 zu tun? Paulus kann das im Partizip angesprochene vergangene Geschehen offenkundig als Mittel für ganz vielfältige Zwecke einsetzen. Und wo diese klar zukünftig gemeint sind, ist solch ein Verständnis auf der

625 Zum prospektiven Konditionalsatz in Röm 14,8 siehe oben, Abschnitt 5.2.2.1.

626 Der Anklang an Gal 2,19-20 ist nicht zu überhören. 
anderen Seite auch meist kontextuell recht deutlich angezeigt. Die Syntax bietet auf jeden Fall keine klare Vorgabe für die Lektüre.

Fee ist einer der wenigen Kommentatoren, der diese Offenheit des Finalsatzes einräumt und explizit bespricht. Er meint, die Ambiguität des ausgedrückten Zwecks - den er als wahrscheinlich sowohl die Gegenwart als auch die Zukunft betreffend ansieht - sei äußerst paulinisch. ${ }^{627}$ Er sieht den Finalsatz motiviert durch den später explizierten Gedanken der Befreiung der Galater aus ihrem Zustand der Versklavung in 4,3 und 9. Entsprechend, meint er, läge wohl auch hier in 1,4 „the emphasis ... on present deliverance. “628 Damit hat er wohl konkreter eine vergangene Befreiung im Sinn, die in einem die Gegenwart betreffenden befreiten Zustand resultierte.

Auch de Boer nimmt diese Formulierung auf: „The grammar of 1,4b ... can be taken to refer to the future (a deliverance that will occur) as well as the present (a deliverance that has occured). “629 Für de Boer kommt hier also die von ihm für Paulus angenommene Situation zum Ausdruck, wonach Gott in Christus einen „eschatological act of cosmic rectification“ begonnen habe, der (hier wieder ganz im Gefolge Käsemann) in einer Kampfsituation bis zur Parusie resultiere. ${ }^{630}$ Entsprechend sieht de Boer in Gal 1,4 - anders als Martyn, welcher die Abgeschlossenheit betont - eine „onging rescuing activity.“631

Es stellt sich die Frage, ob diese Überlegungen von Fee und Martyn tatsächlich aus dem vorliegenden Text ableitbar sind. Die von ihnen vorgenommene Verbindung von geschehener Befreiung mit einem betonten gegenwärtigen Zustand würde, was die Wahl des Aspekts angeht, doch wohl den Konjunktiv Perfekt

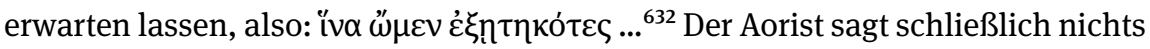
über das Andauern des Zustandes aus, der durch einen vermeintlich vergangenen Akt des Befreiens erreicht worden wäre, ein „Rückfall“ ist hier durchaus möglich. De Boer spricht vom „Gegenwärtigen“ aber auch insofern, als er hier ein andauerndes - das heißt wohl: iteratives - Befreien herausliest. Dann freilich, so könnte man einwenden, hätte Paulus dies durch die Wahl des Konj. Präs. sehr viel deutlicher kommunizieren können. ${ }^{633}$

627 Fee, Galatians, 20.

628 Fee, Galatians, 20.

629 de Boer, Galatians, 36.

630 de Boer, Galatians, 34.

631 de Boer, Galatians, 36.

632 Zum Konjunktiv Perfekt siehe GGNT 203a.

633 Vgl. etwa den Konjunktiv Präsens mit durativem Aspekt bei Plutarch, Mor. 712f: Plutarch meint zur Frage, welche Art Musik zum Gastmahl erlaubt sein solle, dass die Kithara eine derart lange, bereits bei Homer bezeugte Tradition habe. Es sei daher nicht angebracht, diese „Freund-

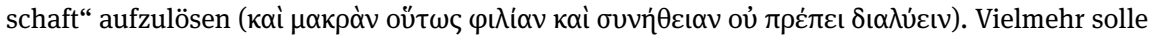


Natürlich schließt die Wahl des Aorists ein solches Verständnis nicht aus. Es muss aber doch erwogen werden, ob nicht zu erwarten wäre, dass Paulus den unmissverständlichen Aspekt gewählt hätte, wenn er derart Spezifisches hätte kommunizieren wollen. Dies gilt umso mehr, als doch festgehalten werden muss, dass die Referenz des vorausgehenden aoristischen Partizips (die Kreuzigung) nahelegt, dass auch das auf dieses Mittel bezogene Ziel am natürlichsten einen spezifischen Akt im Blick hat. Grundsätzlich ist wohl zu beachten, dass der Ver-

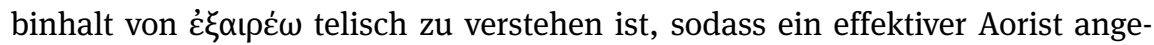
nommen werden könnte. ${ }^{634}$ Worin genau dieser Zielpunkt besteht, womit also ein Bereich außerhalb des gegenwärtigen bösen Äons erreicht wäre, ist schwer zu bestimmen.

Auch wenn man freilich von einem spezifischen Akt, also einem Einzelereignis, ausgeht, ist freilich noch nichts über dessen zeitliche Verortung gesagt. An konkreten kontextuellen Indikatoren, welche eine Verortung in der Vergangenheit plausibilisieren könnten, kommt eventuell die Phrase „nach dem Willen

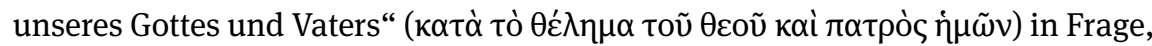
die wohl zum Finalsatz (und nicht zum übergeordneten Partizip, welches bereits eine Präpositionalangabe aufweist) zu rechnen ist. V. 5 greift nun durch einen relativischen Anschluss auf „unseren Vater/Gott“ zurück: „Dem [sei] die Herrlich-

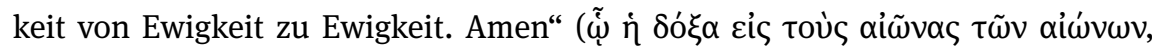
$\dot{\alpha} \mu \eta ́ v)$. Das Lob bezieht sich also nicht direkt auf die (unstrittig bereits geschehene) Hingabe Jesu, sondern auf Gottes Willen, mit diesem Mittel den Zweck des Herausreißens zu verwirklichen. Es könnte sich hierbei durchaus um eine „proleptische“ Doxologie handeln, denn es ist durchaus der Regelfall bei Paulus, dass er in Lob ausbricht, nachdem er ein zukünftiges Heilshandeln Gottes erwähnt hat. ${ }^{635}$ Allerdings ist an jenen Stellen die Zukünftigkeit des Geschehens auch klarer im Text markiert.

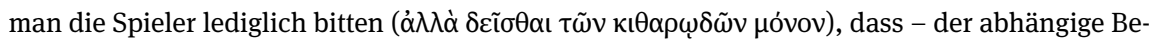
gehrsatz wird mit ö $\omega \varsigma$ ৎ eingeleitet (siehe oben, Abschnitt 4.4.1) - „,ie das viele Jammern und

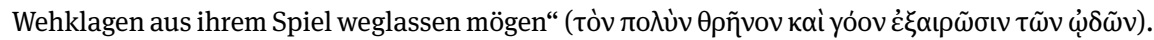
634 Vgl. oben, Kapitel 6, Abschnitt 2.2.2. Ich verdanke diese Überlegung meinem Studenten Jonathan Höfig.

635 Vgl. Eph 3,21 nach 3,29; Phil 4,20 nach 4,19; 2. Tim 4,18. Röm 16,25-26 beinhaltet sowohl einen Verweis auf Vermögen bezüglich zukünftiger Handlungen als auch, dem untergeordnet, einen Rückverweis auf das nun geschehene Offenbarungshandeln. Schwer in dieses Raster einzuordnen ist Röm 11,36. Selbst die Doxologie in 1. Tim 1,17, welche auf die indikativische Feststellung des geschehenen Gnadenerweises Gottes gegenüber Paulus in V. 16 folgt, hat wohl auch die bis in die Zukunft reichende Vorbilds-Funktion im Blick, die vom Erbarmen über Paulus ausgeht. 
Daher könnte man erwägen, ob Paulus die Doxologie hier an so ungewöhnlicher Stelle bringt, ${ }^{636}$ um den Fokus bewusst auf die Frage zu lenken, wofür eigentlich Gott nun gelobt wird. Oder anders ausgedrückt: Wenn das Heilshandeln im Herausreißen aus dem gegenwärtigen Äon besteht (V. 4) und das Lob „,bis in die Äonen der Äonen“ gilt (V. 5), ist derjenige, der in dieses Lob miteinstimmt, dazu aufgefordert, seinen eigenen Standpunkt zu reflektieren. Ein solches Verständnis nimmt sowohl die Grammatik als auch die Pragmatik des Textes ernst und bietet daher die attraktivste Lesweise. Die von Fee richtig wahrgenommene Offenheit der Finalangabe in 1,4 würde Paulus dann als Einstieg in seine Auseinandersetzung mit den Galatern dienen: Die drastische Beschreibung des Zwecks des Todes Jesu fordert gerade zur Reflexion darüber auf, $o b$ dieses Ziel bereits erreicht ist. Es mag durchaus sein, dass die Galater der Gesetzesobservanz zugetan waren, weil in ihrer Wahrnehmung ein solcher Zielpunkt erst noch zu erreichen schien. Paulus hingegen versucht im Brief herauszuarbeiten, dass durch Christus diese Situation tatsächlich bereits erreicht worden ist. Fee ist somit zuzustimmen, dass die vielfache Rede im Brief von einer Überwindung eines früheren Zustands der Unterwerfung unter die „Elemente“ für Paulus den plausibelsten Hintergrund der Finalangabe in 1,4 darstellt.

Damit ist nun freilich nicht gesagt, dass das Erreichen des außerhalb des „gegenwärtigen Zeitalters“ stehenden Bereichs - die Freiheit in Christus - notwendigerweise ein Zustand ist, der noch für die Gegenwart andauert. Letztlich steckt gerade hierin das rhetorische Potenzial der finalen Konnexion in Gal 1,4: Durch die enge Verbindung eines Eintretens in die Sklaverei des Gesetzes mit der vormaligen Unterdrückung durch die Elemente (Gal 4,9) kann Paulus die sich im Verlauf befindliche Hinwendung zum Gesetz als eine Bewegung interpretieren, welche dem Zweck des Sterbens Jesu zugegen läuft und damit auch dieses „Mittel“ verwirft (siehe etwa Gal 2,21). Von einem anhaltenden Rettungshandeln (de Boer) ist deswegen nicht auszugehen. Gerade die Einmaligkeit der bereits erfolgten Rettung macht das Abwenden (vgl. direkt im Anschluss Gal 1,6, wo beim „Rufenden“ vermutlich auch an den Ruf zur Bekehrung gedacht ist) ${ }^{637}$ der Galater so problematisch.

Die von Fee wahrgenommene Verbindung von Gal 1,4 zu Gal 4 ist somit zu bejahen - allerdings auch entsprechend der konkreten Ausgestaltung des letztgenannten Abschnitts entsprechend zu modifizieren: Wie gezeigt (Kapitel 9, Abschnitt 5), liefert Paulus in Gal 4 ja nicht einfach eine feststellend-informierende Erzählung über vergangene Tatsachen, sondern strickt ein Netz aus impliziten,

636 Vgl. Moo, Galatians, 73.

637 Siehe oben, Kapitel 8, Abschnitt 3.3. 
expliziten und explizit in Frage gestellten Handlungssträngen, deren Auflösung letztlich von den Galatern selbst abhängig ist. Wie bereits in Gal 4,9a ist die von Paulus erhoffte Ereignisfolge allerdings auch hier durch die Einbindung der göttlichen Initiative angezeigt.

\subsubsection{Phil 2,10-11 und die Abgrenzung von Zweck-Angabe und zukunftsgewisser Vorausdeutung}

Nachdem die Schwierigkeit der Abgrenzung zwischen zukünftigen und bereits in der Vergangenheit liegenden Zwecken besprochen wurde, soll nun noch kurz auf die teilweise auftretende Problematik eingegangen werden, dass der Zukunftsbezug zwar unstrittig ist, jedoch unklar ist, ob es sich um eine futurische Zweckangabe oder Aussage handelt.

Als Beipieltext dient Phil 2,10-11, wo sowohl die Schwierigkeit einer solchen Entscheidung als auch die Implikationen für das angenommene Protonarrativ deutlich werden. In Phil 2,9 wird die Erhöhung Jesu und die ihm geltende Verleihung des höchsten Namens im Indikativ Aorist erzählt, wobei dieses Handeln

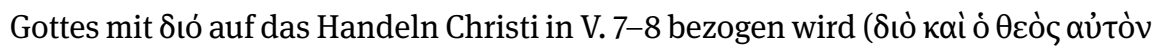

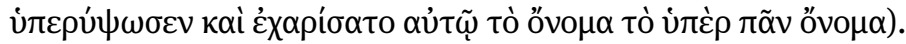

In V. 10 wird dieser Vorgang als Mittel zum mit îva eingeleiteten Zweck markiert: „damit im Namen Jesu jedes Knie sich beuge, der Himmlischen und Irdi-

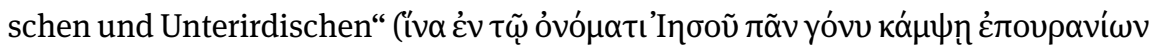

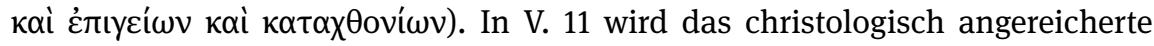
Zitat aus Jes 45,23 fortgesetzt: „... und damit jede Zunge bekenne, dass Jesus

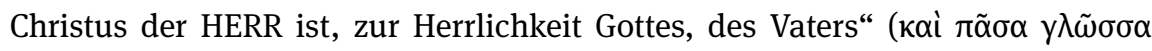

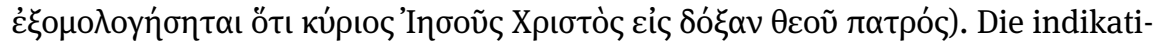

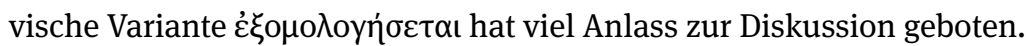

Denkbar ist natürlich, dass mit kaí ein neuer Hauptsatz eingeleitet wird. Man

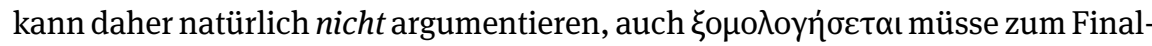

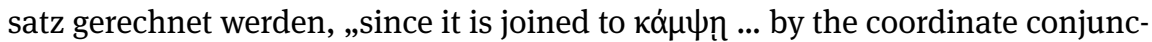

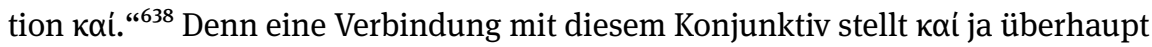
nur her, wenn man bereits die Zugehörigkeit des Verbs von 2,11 zum Finalsatz voraussetzt! Demgegenüber muss festgehalten werden, dass es selbstverständlich

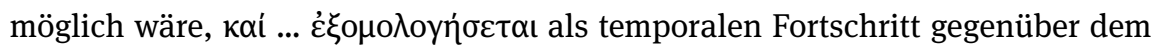
zuvor in V. 9 im Hauptsatz ausgedrückten Handeln Gottes aufzufassen.

Dann wäre gesagt, dass Gott den Christus mit einem bestimmten Ziel erhöhte (V. 9-10) - und dieses (in der eschatologischen Zukunft lokalisierte) Ziel auch tatsächlich erreicht werden wird (V. 11). Dieses Verständnis würde wohl

638 Hawthorne, Philippians, 129. 
voraussetzen, dass das angekündigte Bekennen die Realisierung des dazwischen genannten beabsichtigten Kniebeugens darstellen würde. Insofern das Beugen des Knies und das mündliche Bekennen zwei Schritte einer zusammengehörigen Handlung darstellen, könnte man auch sagen, dass durch die Ankündigung des zweiten die Realisierung des lediglich als beabsichtigt ausgesagten ersten impliziert wäre. ${ }^{639}$

Vermutlich ist V. 11 jedoch in der Tat als Teil der Zweck-Angabe zu betrachten - und zwar völlig unabhängig davon, ob man Konjunktiv Aorist oder Indikativ Futur liest. Denn aufgrund der lautlichen Ähnlichkeit von o/ $\omega$ und (vermutlich) $\varepsilon \mathrm{\varepsilon} / \eta$ hatte sich die Unterscheidung zwischen Indikativ Futur und Konjunktiv Aorist zur Zeit der Abfassung des NTs auch dort zu einer vor allem orthographischen Angelegenheit entwickelt, wo sich die Ausgänge noch lautlich unterschieden, ${ }^{640}$ sodass der Indikativ des Futurs auch häufiger nach iv $\alpha$ auftreten konnte (siehe etwa Gal 2,4).

Die Frage, ob eine Futurform nach vorausgehendem ivvo-Satz mit Konjunktiv einen neuen Hauptsatz bildet oder als Fortführung des Nebensatzes zu lesen ist, stellt sich daher an zahlreichen Stellen im NT - und nicht immer ist eine klare Antwort möglich. ${ }^{641}$ Erstaunlicherweise wird diese Option gerade in der

639 Deutsches Beispiel: „Ich werde Dir Geld geben, damit Du Brot kaufst. Und Du wirst mir das Wechselgeld zurückgeben." Vgl. oben, Kapitel 8, Abschnitt 3 zur Ellipse.

640 Vgl. hierzu Caragounis, Development, 556-667. Die lautliche Entwicklung von $\eta$ ist natürlich umstritten (siehe oben, Abschnitt 2.1).

641 Beispielsweise kann bei Lk 22,30 kann mit gutem Recht gefragt werden, ob nach dem vermutlich konsekutiven ïvo zwei oder drei Verben im Nebensatz zu lesen sind: „dass ihr esst und trinkt an meinem Tisch in meinem Reich und sitzt(/. Und ihr werdet sitzen) auf Thronen, um

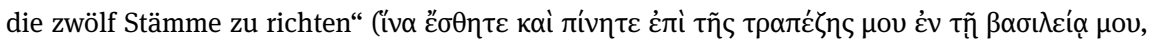

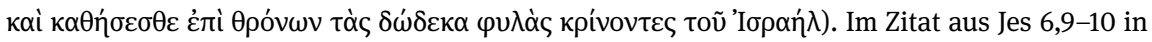

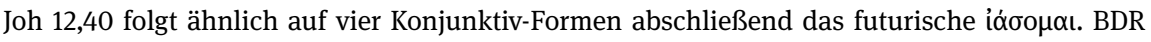
$442^{8}$ schreiben hierzu: „Durch кaí wird die Folge gewissermaßen verselbstständigt, ohne dabei aber ein selbstständiger Satz zu werden.“ Es scheint aufgrund von Stellen wie Joh 6,39 jedoch fraglich, die Verwendungsweise am kai festzumachen. Etwas verwirrend zu dieser Stelle ist Schnackenburg, Johannesevangelium 73-74: Zu 39 meint er, das „letzte Sätzchen“ wirke „wie angehängt und überflüssig.“ Offenbar versteht er es als selbstständigen Hauptsatz, wenn er es hier übersetzt als: „Ich werde ihn auferwecken am letzten Tag.“ In der Übersetzung des Abschnittes (Schnackenburg, Johannesevangelium II, 70) schreibt er jedoch: „39 Das aber ist der Wille dessen, der mich gesandt hat, daß ich nichts von dem, was er mir gegeben hat, verlorengehen lasse, sondern daß ich es auferwecke am letzten Tag. 40 Denn das ist der Wille meines Vaters, daß jeder, der den Sohn sieht und an ihn glaubt, ewiges Leben hat und ich ihn auferwecke am letzten Tag.“ In der Tat liest sich das $\alpha \lambda \lambda \alpha \dot{~ i n ~ V . ~} 39$ am natürlichsten als Anfügung des positiven Willens Gottes:

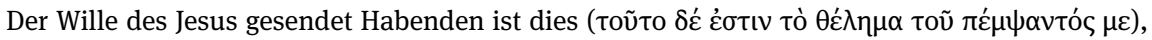
„dass ... ich niemanden von ihnen [zum hier umschifften Anakoluth siehe Robertson, Grammar,

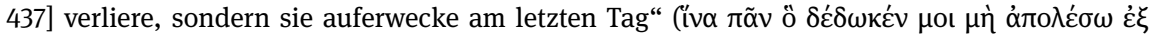


Auslegung der Offenbarung meist nicht genügend berücksichtigt. So schreibt Aune zu Offb 2,10, dass der Wechsel in das Futur „significant“ sei. ${ }^{642}$ Dass diese Aussage nicht näher begründet wird, ist erstaunlich, denn in Offb 9,5 bietet der Autor in einer Negativum-Positivum-Relation zwei ǐva-Sätze (zur Explikation des Gebots), wobei der erste im Konjunktiv steht, der zweite im Indikativ Futur (iv $\alpha$

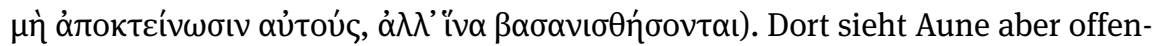

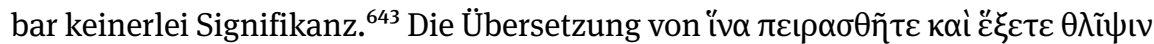
$\dot{\eta} \mu \varepsilon \rho \tilde{\omega} \nu \delta \delta^{\prime} \kappa \alpha$ in Offb 2,10 als ,that you might be tested, and you will experience tribulation for ten days“ zeigt demgegenüber, dass er hier das Futurverb einem neuen Hauptsatz zurechnet. Eine solche Aufteilung ließe sich allenfalls inhaltlich begründen. ${ }^{644}$

Auch die Entscheidung dafür, dass V. 11 ebenfalls von ǐv $\alpha$ abhängt, fällt daher letztlich auf der Grundlage, dass es keinen Anlass gibt, einen Übergang von „lediglich“ Bezwecktem zu tatsächlich zukünftig Geschehendem zu erkennen. Hätte Paulus einen solchen Übergang markieren wollen, hätte er V. 11 vermutlich mit einer temporalen Angabe (z. B. „,an jenem Tag“) ${ }^{645}$ oder einer die Wirklich-

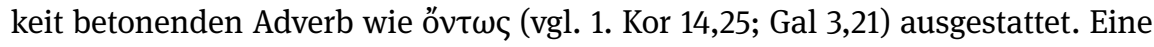
derartige Markierung könnte hier mit gutem Recht vom Leser erwartet werden, bedenkt man, dass in der Vorlage beide Verben Teil desselben ö $\tau$-Satzes und dort

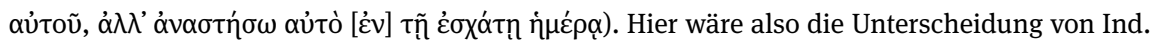
Fut. und Konj. Aor. nach ïva im NT wohl nur noch eine Sache der Orthographie. Dasselbe muss natürlich ebenso für V. 40 bedacht werden, wo Ind. Fut. auf Konjunktiv (Präsens) mit kaí verbun-

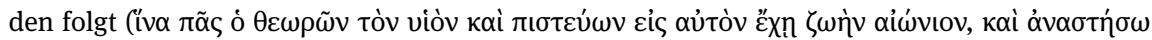

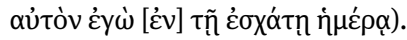

642 Aune, Revelation I, 166.

643 Aune, Revelation II, 530.

644 Im Hauptsatz steht die Ankündigung, der Teufel werde einige der Angeschriebenen in das

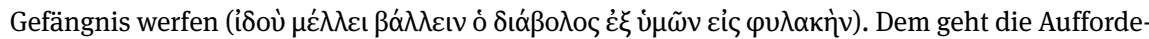
rung voraus, sich nicht vor dem zu Fürchten, was an Leiden auf „dich“ zukommt ( $\mu \eta \delta \varepsilon \dot{v}$ $\varphi$ oßoṽ

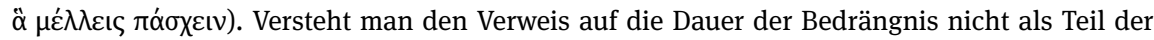
Absicht des Teufels, sondern als Versicherung, dass diese nur kurze Zeit dauern werde, wird dadurch in der Tat eine Motivation der Aufforderung geboten. Ebenfalls lässt sich in Offb 19,15 lediglich ein inhaltliches Argument vorbringen: Das scharfe Schwert geht aus dem Mund hervor,

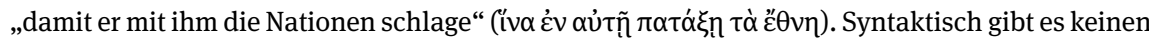

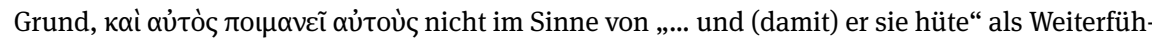

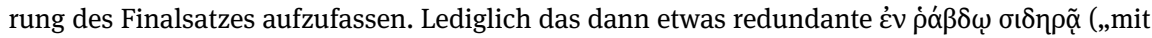
eisernem Stab“) spricht gegen eine solche Auslegung: Denn das Schwert aus dem Mund kann nur schwer als Mittel dazu verstanden werden, dass eine andere Handlung mit dem eisernen Stab erfolgt.

645 Vgl. mit lokaler Angabe Mt 28,10: „Verkündet meinen Brüdern, dass sie nach nach Galiläa

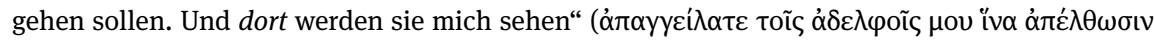

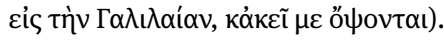




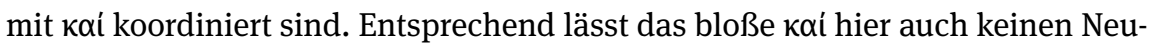
ansatz erwarten. ${ }^{646}$

Damit ist freilich keinesfalls gesagt, dass „one can come to the conclusion that all will confess Jesus as Lord only by choosing the ... (future indicative: , will openly acknowledge') over the ... aorist subjunctive ,might openly acknowledge“ ... and by breaking v11 free from v 10, thereby making v11 an independent clause that asserts what will unquestionably happen in the future. “647 Richtig wäre, dass nur dann das Eintreffen als tatsächlich eintretend ausgesagt wäre - was aber natürlich nicht bedeutet, dass Paulus das Erreichen der Zweck-Proposition für zweifelhaft hält. Im Gegenteil demonstriert Röm 14,11, dass Paulus denselben Text durchaus als eschatologische Ankündigung versteht (oder zumindest verwenden kann). Dort gebraucht Paulus den ursprünglichen Indikativ Futur, um auf den Umstand $\mathrm{zu}$ verweisen, dass sich jeder vor dem göttlichen Gericht verantworten werden müsse. ${ }^{648}$ Liest man Phil 2,10-11 vor diesem Hintergrund, wird deutlich, dass Paulus schlicht deswegen nun im Fall des ersten Verbs statt

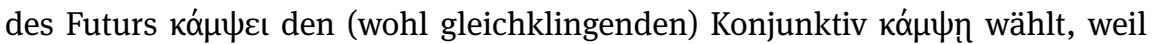
dieses zukünftige Geschehen nun als Zweck-Proposition zu einer anderen Handlung tritt. Dass diese in der Vergangenheit anzusiedeln ist, ändert nichts an der Zukunftsreferenz des Ziels aus der Perspektive des Autors.

Es ist daher gänzlich unangebracht, mit Käsemann hier eine „kosmisch[e] Proskynese “649 im Moment der Inthronisation durch die Geistermächte anzunehmen, nur weil ${ }^{650}$ „das Futur der alt. Aussage in unserem Text offensichtlich nicht grundlos fallengelassen ist.“651 Der Indikativ des Futurs wurde nicht „fallengelassen“ - weder zur Eliminierung des Zukunftsbezugs, noch zur Abschwächung der

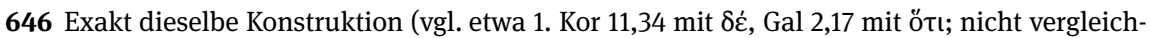
bar ist Eph 6,21) wie in Phil 2,10-11 liegt im Übrigen auch in Eph 6,3 vor im Zitat aus Exod 20,12/ Deut 5,16, welches natürlich nicht zu übersetzen ist als ${ }^{\star}, \ldots$... damit es Dir gut ergehe. Und Du wirst

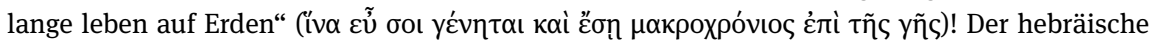

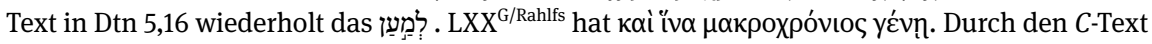
ist auch das Futur हैon mit zweitem îva für Exod 20,12 belegt.

647 Hawthorne, Philippians, 129.

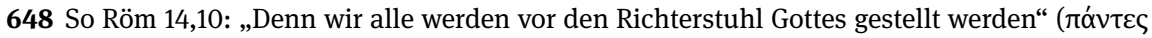

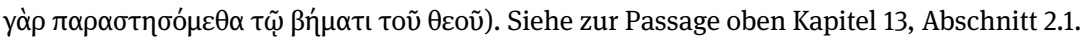

649 Käsemann, „Kritische Analyse,“ 85.

650 Käsemann, „Kritische Analyse,“ 85 verweist auf das zusammengetragene religionsgeschichtliche Material von Dibelius Philipper, 79. Er schreibt jedoch auch (Käsemann, „Kritische Analyse,“ 86), den „Ausschlag“ gäbe der Konjunktiv.

651 Käsemann, „Kritische Analyse,“ 86. 
Aussage, sondern aufgrund des zusätzlichen inhaltlichen Elements der finalen Konnexion. ${ }^{652}$

Die Wahl der finalen Konnexion anstelle der konsekutiven Konnexion (theoretisch denkbar wäre natürlich auch, das tatsächlich vorliegende ǐvo konsekutiv zu verstehen) ist also nicht einfach eine Abschwächung, da in der finalen Konnexion der zusätzliche Aspekt der Intention zum Ausdruck gebracht werden kann. Andersherum kann in bestimmten Kontexten - wie dem hier anzutreffenden das Erreichen der Ziel-Proposition einer finalen Konnexion aufgrund des sachlichen Zusammenhangs schlicht vorausgesetzt sein. ${ }^{653}$

Im Hinblick auf Phil 2,10-11 lässt sich für Protoerzählungen daher dreierlei ableiten. Einerseits ist darauf $\mathrm{zu}$ insistieren, dass nach ǐvo-Satz ein Futur-Verb nach кoí durchaus nicht notwendigerweise als zukunftsgewisse Vorausdeutung gelesen werden muss. In den Versen 10-11 liegt wohl kein Übergang von bloß beabsichtigtem Kniebeugen zu tatsächlich angekündigtem Bekennen vor.

Zugleich zeigt sich aber auch, dass die Wahl einer Fokalisierungsinstanz in der Vergangenheit - hier: Gott - und die Nennung eines von diesem Standpunkt verfolgten Zwecks nicht unbedingt mit der Absicht der Abschwächung des Protonarrativs erfolgen muss. Zukunftsungewisse Vorausdeutungen können kontextuell denselben Zweck erfüllen, wie zukunftsgewisse Ankündigungen. Für den „Proto-Erzähler“ attraktiv ist jedoch, dass zugleich auch der zusätzliche Aspekt der Intention der Fokalisierungsinstanz kommuniziert werden kann. Die Nähe zur Ellipse nach Nennung vergangener Zwecke (vgl. Gal 1,18) ist hier sehr auffällig.

Hier wie dort ist dann freilich auch Hintergrundwissen vorausgesetzt, was ein adäquates Verständnis erlaubt. In Gal 1,18 betrifft dies Kenntnisse der etablierten sozialen Interaktionsmuster. In Phil 2,10-11 sind wohl einerseits Vorannahmen zur Effektivität des göttlichen Handelns (vgl. V. 9) vorausgesetzt, aller Wahrscheinlichkeit nach aber auch konkrete futurische „Heilsgeschichten,“ die Paulus in Philippi erzählt haben wird und die auch das Eschaton betroffen haben werden.

652 Richtig Wallace, Grammar, 474.

653 Es ist fraglich, ob deswegen zum finalen und manchmal vorkommenden konsekutiven Gebrauch von ǐva noch eine zusätzliche Kategorie eines final-konsekutiven ìv angenommen werden sollte. So Wallace, Grammar, 473 („Purpose-Result”). Er räumt jedoch auch ein: „This probably does not represent a change in syntax from classical to Koine, but a change in subject matter. It is, of course, possible to treat each of these examples as simply purpose iv $\alpha$ clauses in which there is evidently no doubt about the accomplishment from the speaker's viewpoint. Hence, in order that is an acceptable gloss." 